\title{
Frequency Offset Tolerant Demodulators for UNB Communications
}

\section{Siavash Safapourhajari}

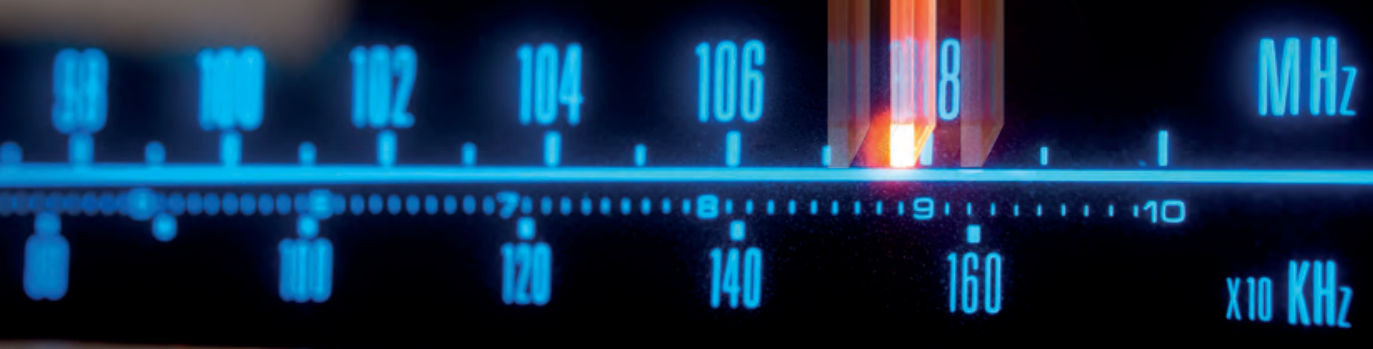





\section{Frequency Offset Tolerant Demodulators for UNB Communications}


Members of the graduation committee:

Dr. ir. A. B.J. Kokkeler University of Twente (promotor)

Prof. dr. ir M.J. G. Bekooij University of Twente

Prof. dr. ir G. J. Heijenk University of Twente

Prof. dr. ir C. H. Slump University of Twente

Prof. dr. ir M.J. Bentum

Dr. ir. G.J.M. Janssen

Prof. dr. J. N. Kok

Eindhoven University of Technology

Delft University of Technology

University of Twente (chairman and secretary)

\section{UNIVERSITY OF TWENTE.}

Faculty of Electrical Engineering, Mathematics and Computer Science, Computer Architecture for Embedded Systems (CAES) group/ Radio Systems (RS) group

\section{DIGITAL SOCIETY INSTITUTE}
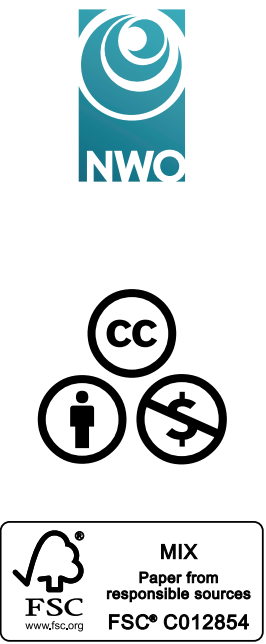

DSI Ph.D. Thesis Series No. 20-006

Digital Society Institute

PO Box 217, 7500 AE Enschede, The Netherlands

This research has been conducted within the Slow Wireless project (project number 13769). This research is supported by the Dutch Research Council NWO.

Copyright ${ }^{\odot} 2020$ Siavash Safapourhajari, Enschede, The Netherlands. This work is licensed under the Creative Commons Attribution-NonCommercial 4.० International License. To view a copy of this license, visit http://creativecommons.org/ licenses/by-nc/4.0/deed.en_US.

This thesis was typeset using $\mathrm{L}_{\mathrm{E}}^{\mathrm{T} X} \mathrm{X}$ and TikZ. This thesis was printed by IPSKAMP printing, The Netherlands.

ISBN 978-90-365-5086-4

ISSN 2589-7721; DSI Ph.D. Thesis Series No. 20-006

DOI $10.3990 / 1.9789036550864$ 


\title{
Frequency Offeset Tolerant Demodulators for UNB COMMUNicATIONS
}

\author{
DisSERTATION
}

to obtain

the degree of doctor at the University of Twente, on the authority of the rector magnificus, prof. dr. ir. A. Veldkamp, on account of the decision of the Doctorate board to be publicly defended

on Friday the $11^{\text {th }}$ of December 2020 at $14: 45$ hours

by

Siavash Safapourhajari

born on the $22^{\text {nd }}$ of September 1987

in Bandaranzali, Iran 
This dissertation has been approved by:

Dr. ir. A.B. J. Kokkeler (promotor)

Copyright ${ }^{\circledR} 2020$ Siavash Safapourhajari ISBN 978-90-365-5086-4 
To my love Soheila, To my parents and my brother 


\section{Abstract}

Interconnected temperature sensors in a wine cellar, smart meters in houses and wearable sensors monitoring health status are all examples of Wireless Sensor Networks (WSN). Thanks to the progress in wireless communications and networking technologies as well as developments in electronic design, it is possible to deploy numerous low power wirelessly connected devices and sensors for a variety of applications. Nevertheless, improving energy efficiency for power constrained wireless nodes is a never-ending quest. Providing wireless communication for diverse applications with different requirements has led to the emergence of different types of wireless networks. A recently emerged type of wireless networks is Low Power Wide Area Network (LPWAN) which provides a wide coverage $(10-50 \mathrm{~km}$ in rural areas and $1-5 \mathrm{~km}$ in Urban areas) and low power communication for low data rate applications.

Different technologies including Ultra-Narrowband (UNB), Spread Spectrum and Narrowband-IoT have been introduced for realizing LPWANs. Among these, UNB communication is one of the interesting candidates as it uses unlicensed spectrum, performs better in presence of interference and provides low cost and wide coverage. The Slow Wireless project focuses on physical layer aspects to investigate UNB communications in the (sub) GHz ISM band. As one of the work packages in the Slow Wireless project, the current thesis aims at digital signal processing techniques for UNB communication nodes. Although the very low data rate in UNB communication relaxes some aspects of the transceiver design (such as lower clock frequencies in the baseband processing), it poses other challenges. One of the prominent challenges in UNB communications is Carrier Frequency Offset (CFO).

CFO might be a consequence of the mismatch between oscillators in the transmitter and the receiver or the Doppler shift caused by the relative movement of the nodes. As a solution to the CFO problem, offset tolerant demodulators have been proposed to replace precise but power-hungry carrier frequency recovery. Most of these methods assume that the CFO is in the same order of the signal bandwidth. Then, instead of precise carrier recovery, an offset tolerant demodulator is used which can tolerate the frequency offset without deteriorating the detection performance. However, in UNB communications using low cost crystals, the resulting CFO can become several times the signal bandwidth. In some UNB systems (e.g. Sigfox) solutions in the network layer are adopted to solve the CFO problem. However, it is not sufficient when low cost crystals are used, and it still places limitations on the communication system design. 
To overcome the CFO problem in UNB communication, offset tolerant demodulators are considered in this thesis while focusing on achieving scalable offset tolerance. In other words, the demodulator should obtain the same BER value for a certain $E_{b} / N_{0}$ regardless of how large the frequency offset is. Of course, it is assumed that the filter prior to the demodulator is wide enough to capture the signal in presence of a large CFO. In this thesis, first, the performance of two offset tolerant demodulators for FSK and PSK, which are potentially suitable for scalable offset tolerance, are investigated to find their limitations when a large CFO tolerance is needed. For FSK modulation, a DFT-Based demodulator is designed by modifying an existing demodulator. On the other hand, for PSK, an Auto-Correlation Demodulator (ACD) for Double Differential PSK is used which can tolerate CFO. Two aspects are considered for scalable offset tolerance; the BER performance of the demodulator and how the complexity of the demodulator scales when the range of the tolerable offset increases. It is shown that for the DFT-based FSK and the DDPSK demodulator, the complexity and the BER performance are limiting factors, respectively.

To circumvent the limitations of the demodulators for FSK and PSK and to achieve scalable offset tolerance, two different algorithms are proposed. The complexity of the DFT-based demodulator is due to a high complexity window synchronization. Hence, a low complexity window synchronization algorithm is introduced with an efficient implementation. The complexity of the proposed design scales more efficiently compared to the conventional method when tolerable CFO increases. Furthermore, the performance of the DDPSK demodulator degrades as a result of increased noise bandwidth. The increased noise bandwidth is a consequence of increasing the bandwidth of the filter prior to the demodulator which is required in presence of a large frequency offset. To tackle this problem, a demodulator based on shifted correlation is proposed for DDPSK modulated signals. Using this demodulator, the effect of increased noise bandwidth is removed and the BER performance will be independent of the range of the tolerable CFO.

In addition to the $\mathrm{CFO}$, temporal fading in $\mathrm{UNB}$ communications must be taken into consideration. Very low data rate communication systems are vulnerable to a time-varying fading channel. Even for nodes at fixed positions in $\mathrm{UNB}$, the movement of surrounding objects can lead to a time-varying fading channel and degrade BER performance. To combat distortion in a time-varying channel, time and frequency diversity techniques together with channel coding and interleaving can be utilized. To afford the redundancy required for these solutions, a higher bitrate is required. Higher order PSK and FSK modulation considerably compromise energy and bandwidth, respectively. Frequency/phase modulation (FPSK) is a method which can increase the bitrate in a more power and bandwidth efficient way than PSK and FSK, respectively.

To alleviate the effect of CFO and temporal fading simultaneously, FPSK modulation is considered in this thesis. As a solution for CFO, an offset tolerant 
demodulator is proposed for FPSK modulation. Moreover, the performance of this modulation is considered in a time-varying channel while using a system designed for including time diversity. The BER performance of the proposed demodulator is evaluated for different combinations of FSK and PSK modulation orders. Using different scenarios, it is demonstrated that the proposed offset tolerant demodulator for FPSK using time diversity can improve the BER performance in a time-varying channel. 


\section{SAMENVATTING}

Onderling verbonden temperatuursensoren in een wijnkelder, slimme meters in huizen en draagbare sensoren die de gezondheid van een patient bewaken zijn allemaal voorbeelden van Wireless Sensor Networks (WSN). Dankzij vooruitgang in draadloze communicatie- en netwerktechnologieën en ontwikkelingen in electronisch-ontwerp technieken, is het mogelijk om talloze draadloos verbonden apparaten en sensoren met laag vermogen in te zetten voor een verscheidenheid aan toepassingen. Niettemin blijft het verbeteren van de energie-efficiëntie voor draadloze sensoren met beperkte stroomvoorziening een nooit eindigende zoektocht. Het inzetten van draadloze communicatie voor diverse toepassingen met een grote verscheidenheid aan eisen heeft geleid tot de opkomst van verschillende soorten draadloze netwerken. Een recente ontwikkeling in draadloze netwerken is Low Power Wide Area Network (LPWAN) dat een brede dekking biedt (10-50 km in landelijke gebieden en $1-5 \mathrm{~km}$ in stedelijke gebieden) en dit combineert met een laag stroomverbruik voor communicatietoepassingen met lage gegevenssnelheden.

Om LPWAN's te realiseren zijn er verschillende technologieën geïntroduceerd, waaronder Ultra-Narrowband (UNB), Spread Spectrum en Narrowband-IoT. Van deze technologieën is UNB-communicatie één van de interessantste kandidaten, omdat het vergunningsvrij spectrum gebruikt, beter presteert in aanwezigheid van interferentie, lage kosten heeft en een brede dekking biedt.

Het Slow Wireless-project richt zich op UNB-communicatie in de (sub) GHz ISM-band met als doel om aspecten van de fysieke laag te onderzoeken. Dit proefschrift richt zich op één van de werkpakketten in het Slow Wireless-project: digitale signaalverwerkingstechnieken voor UNB-communicatie. Waar de zeer lage gegevenssnelheid in UNB-communicatie sommige ontwerpaspecten van de zendontvanger vereenvoudigt (zoals lagere klokfrequenties in de basisbandverwerking), worden juist andere uitdagingen geïntroduceerd. Een van deze belangrijkste uitdagingen bij UNB-communicatie is Carrier Frequency Offset (CFO).

CFO kan een gevolg zijn van de verschillen tussen de draaggolffrequenties die gegenereerd worden door de oscilatoren in de zender en de ontvanger of van de Dopplerverschuiving veroorzaakt door de relatieve beweging van de node. Als oplossing voor het CFO-probleem zijn offset-tolerante demodulatoren voorgesteld om de precieze maar energie slurpende afstemming van draaggolffrequenties te vervangen. Bij de meeste van deze methoden wordt ervan uitgegaan dat de 
CFO de zelfde grootte heeft als de signaalbandbreedte. Vervolgens wordt in plaats van een nauwkeurig draaggolfherstel een offset-tolerante demodulator gebruikt die de frequentieverschuiving kan tolereren zonder de detectieprestatie te verslechteren. In UNB-communicatie waarbij goedkope kristallen worden gebruikt, kan de resulterende $\mathrm{CFO}$ echter meerdere keren de signaalbandbreedte worden. In sommige UNB-systemen (bv. Sigfox) worden oplossingen in de netwerklaag gebruikt om het CFO-probleem op te lossen. Dit is niet voldoende wanneer goedkope kristallen worden gebruikt en het legt nog steeds beperkingen op aan het communicatiesysteemontwerp.

Om het CFO-probleem in UNB-communicatie op te lossen, worden in dit proefschrift offset-tolerante demodulatoren beschouwd, waarbij de nadruk ligt op het bereiken van een schaalbare offset-tolerantie. Met andere woorden, de demodulator zou dezelfde Bit Error Rate (BER) waarde moeten hebben voor een bepaalde Signaal-Ruis verhouding (SNR) ongeacht de grootte van de frequentieverschuiving. Natuurlijk wordt aangenomen dat het filter voorafgaand aan de demodulator breed genoeg is om het signaal op te vangen in aanwezigheid van een grote CFO. In dit proefschrift worden eerst de prestaties van twee offset tolerante demodulatoren voor Frequency Shift Keying (FSK) en Phase Shift Keying (PSK), die mogelijk geschikt zijn voor schaalbare offset tolerantie, onderzocht om hun beperkingen te vinden wanneer een grote $\mathrm{CFO}$ tolerantie nodig is. Voor FSK-modulatie wordt een Discrete Fourier Transform (DFT) gebaseerde demodulator ontworpen door een bestaande demodulator te wijzigen. Voor PSK wordt een Auto-Correlation Demodulator (ACD) voor Double Differential PSK (DDPSK) gebruikt die CFO kan verdragen. Twee aspecten komen in aanmerking voor schaalbare offset-tolerantie: de BER-prestatie van de demodulator en hoe de complexiteit van de demodulator schaalt als het bereik van de toelaatbare offset toeneemt. Het is aangetoond dat voor de DFT gebaseerde demodulator voor FSK en de DDPSK-demodulator de complexiteit respectievelijk de BER-prestaties beperkende factoren zijn.

Twee verschillende algoritmen worden voorgesteld om de beperkingen van de demodulatoren voor FSK en PSK te omzeilen en een schaalbare offset-tolerantie te bereiken. De complexiteit van de DFT gebaseerde demodulator wordt veroorzaakt door de hoge complexiteit van de symbool synchronisatie. Een algoritme voor venstersynchronisatie met lage complexiteit en een efficiënte implementatie wordt geïntroduceerd. De complexiteit van het voorgestelde ontwerp schaalt efficiënter in vergelijking met de conventionele methode bij toenemende maximale CFO. Bij de DDPSK-demodulator nemen de prestaties af als gevolg van een grotere ruisbandbreedte. De toegenomen ruisbandbreedte is een gevolg van het vergroten van de bandbreedte van het filter voorafgaand aan de demodulator, wat is vereist bij aanwezigheid van een grote frequentieverschuiving. Om dit probleem te adresseren, wordt een demodulator op basis van een verschoven correlatie voorgesteld. Met deze demodulator wordt het effect van een grotere ruisbandbreedte opgeheven en zijn de BER-prestaties onafhankelijk van het bereik van de aanvaardbare CFO. 
Naast de CFO moet ook rekening worden gehouden met tijdsafhankelijk kanaalcondities in UNB-communicatie. Communicatiesystemen met zeer lage datasnelheid zijn kwetsbaar voor een in de tijd variërend kanaal. Zelfs voor knooppunten op vaste posities in UNB kunnen de bewegingen van omringende objecten leiden tot een in de tijd variërend kanaal en zo de BER-prestaties verslechteren. Om vervorming in een in de tijd variërend kanaal te bestrijden, kunnen tijd- en frequentie-diversiteitstechnieken samen met kanaalcodering en interleaving worden gebruikt. Om de voor deze oplossingen vereiste redundantie te verkrijgen, is een hogere bitsnelheid vereist. Hogere orde PSK- en FSKmodulatie zijn echter niet energie-, respectievelijk bandbreedte efficient. Een combinatie van frequentie- en fasemodulatie(FPSK) is een methode die de bitrate op een meer energie- en bandbreedte-efficiënte manier kan verhogen dan respectievelijk PSK en FSK.

Om de effecten van CFO en een tijd variërend kanaal tegelijkertijd te verzachten, wordt in dit proefschrift gebruik gemaakt van FPSK-modulatie. Als oplossing voor $\mathrm{CFO}$ wordt een offset-tolerante demodulator voor FPSK-modulatie voorgesteld. Bovendien wordt de prestatie van deze modulatie in een in de tijd variërend kanaal beschouwd. De BER-prestatie van de voorgestelde demodulator wordt geëvalueerd voor verschillende combinaties van FSK- en PSK-modulatieordes. Met behulp van verschillende scenario's wordt aangetoond dat de voorgestelde offset-tolerante demodulator voor FPSK die gebruikmaakt van tijddiversiteit de BER-prestaties in een in de tijd variërend kanaal kan verbeteren. 


\section{ACKNOWledgments}

This is the last page of another chapter in my life. A journey full of excitement, frustration, sadness, happiness and accomplishments. Going through this was not easy; however, I was privileged to have incredible people around me who made it possible. Promoter, colleagues, friends and family who provided me with guidance, support and love; all I needed to move towards my goals.

First and foremost, I would like to express my profound gratitude towards my promotor, Dr. André Kokkeler. Once he told me that supervising $\mathrm{PhD}$ candidates is somehow similar to raising kids. They should have freedom to find their way and even feel frustrated (just enough) but should be guided before they lose the track. Using his immense knowledge, invaluable experience and endless patience, André implemented this idea in the best way possible and has become a brilliant mentor for me. He was not just a supervisor (at least not what I had in mind) but an open-minded colleague who is always available for providing guidance and discussing ideas. Dear André! Thank you for giving me this opportunity, sharing your knowledge, asking critical questions and offering encouragements when I needed them the most.

Furthermore, I would like to show my appreciation to the graduation committee members for their review and suggestions for improving this manuscript. Looking one step back before the start of my $\mathrm{PhD}$, I would also like to thank Dr. Erik Klumperink and Prof. Bram Nauta for their trust and for being such a considerate professionals. I would like to thank all the members of the Slow Wireless project supervision team Dr. Ronan van der Zee, Dr. Arjan Meijerink, Prof. Mark Bentum and Dr. Andrés Alayon Glazunov for their critical views. I am also grateful for the comments and feedback from the user committee members of the Slow Wireless project. Moreover, I wish to thank two bright PhD candidates Zaher Mahfouz and Joeri Lechevallier with whom I collaborated in the Slow Wireless project.

Right after starting my $\mathrm{PhD}$ I understood a basic rule, a reliable secretary is one of the pillars of a successful group. I wish to thank Marlous Weghorst and Nicole Baveld who helped me during this period. I would like to offer special thanks to my first office-mate, my friend Jordy Huiting. Thank you Jordy! For all fruitful talks and discussions, for helping me to integrate into the group and know more about the Dutch culture; I learned a lot from you. When moving from Iran to another country, what is better than having fellow Iranians in the office next door? I want to thank Hassan Ebrahimi and Masoud Abbasi Alaei 
for all the tips and talks. Moreover, I wish to thank Ghayoor Gillani for his help, particularly, during the final preparation of this thesis as well as Guus Kuipers for making this template available. Spending eight hours a day in office becomes pleasant with cool office-mates and for that I would like to thank Oguz Meteer and Anuradha Chathuranga Ranasinghe.

My first culture shock (!) was the concept of lunch walk, which practically did not include much lunch! It was an example of amazing activities in the CAES group with lots of fun moments and enlightening discussions; I would like to thank all CAES members who made this fantastic atmosphere. Specially, I would like to give credit to Gerwin, Hendrik, Marco (Gerards) and Bert for their persistence and commitment to keeping coffee breaks, lunch walks and Friday drinks up and running. During my PhD, I worked with fabulous MSc. and BSc. students including Zhiyuan, Giovanni, Victor, Marko, Pieter, Torben and Stefan who helped me further investigate my research topic.

In the last months of preparing this thesis, I moved to the Radio Systems (RS) group. It was/is a remarkable experience and I would like to thank all RS members for this; particularly, Lillian, Andrés, Yang, Zaher and Anastasia. Furthermore, I wish to show my appreciation to Chris Zeinstra for helping me with preparing the Samenvatting of this thesis.

Far away from our families and close friends in Iran (whom we miss so much), Soheila and I were lucky to find friends here who supported us and became as family to us. Special thanks to my paranymphs Sajjad Rahimi-Ghahroodi and Neda Bayat for standing by me in my defense. I would like to thank Shirin, Mohammad (Bazr Afshan), Mohammad (Mozzafari), Neda (Mostafa), Zahra, Pouya, and all our friends who made this period a delightful time.

I have become who I am owing to my first role-models. My parents who gave me love, taught me life, inspired me, accepted my decisions and supported me when I felt broken. Mom and dad! when I look back at all the memories we share, all happy and sad moments, I cannot wish for better parents than you. I would also like to thank my lovely little brother (not so little anymore!) who changed my life in the best way. Thank you Nariman! for all the fun, heated discussions and even sibling fights (!) we had. I would also like to tell my beloved in-laws (Parvin, Manoucheher, Siamak, Siavash, Laleh, Yasamin and Babak) how grateful I am for their kindness and support.

The last but not least, I would like to express my heartfelt gratitude towards my friend and my love, Soheila. Darling! it has been more than 13 years since the very first day we started our adventure (the memories of which I vividly remember). Since then, I got unconditional love and support from you no matter how far away we were from each other or how tough the life was. I could not have done this without you and I wish I could thank you enough.

Siavash

Enschede, December 2020 


\section{Contents}

xvii

1 INTRODUCTION 1

1.1 Massive Connectivity ... . . . . . . . . . . . . . . 1

1.1.1 Low Power Wide Area Networks (LPWAN) .......... 2

1.1.2 Ultra-Narrowband (UNB) Communication .......... 3

1.1.3 The Slow Wireless Project . . . . . . . . . . . . . . 4

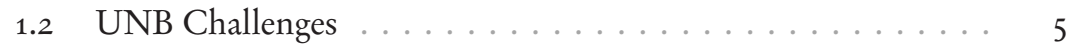

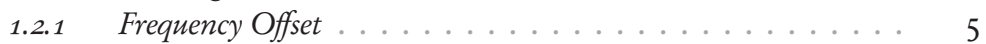

1.2.2 Scalable Offset Tolerance ................. 6

1.2.3 Temporal Fading and Offset Tolerant Demodulators ...... 7

1.3 Research Objectives ... . . . . . . . . . . . . . 8

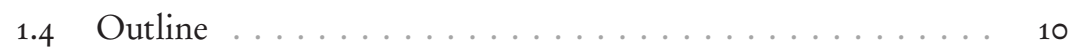

2 BACKGROUND 13

2.1 System Model and Complex Notations . . . . . . . . . . . . 13

2.2 Frequency Shift Keying (FSK) Modulation . . . . . . . . . 15

2.3 Phase Shift Keying (PSK) Modulation ... . . . . . . . . 18

$2.3 .1 \quad$ Differential PSK (DPSK) . . . . . . . . . . 18

2.4 Hybrid Modulation ... . . . . . . . . . . . . . 20

2.4.1 Quadrature Frequency/Phase Modulation(QFPM) ....... 20

2.4.2 Frequency/Phase Shift Keying (FPSK) ............ 21

2.5 Frequency Offset; Compensate or Tolerate ... . . . . . . . 22

2.5 .1 Compensating Frequency Offset ............. 23

$2.5 .2 \quad$ Tolerating Frequency Offset . . . . . . . . . . . . 24

3 Offset Tolerant Demodulators for UNB Communica$\begin{array}{ll}\text { TIONS } & 27\end{array}$

3.1 Introduction . . . . . . . . . . . . 27

3.2 Related Work ... . . . . . . . . . . . . . . . . . 29

3.3 The DFT-based Demodulator ............... 30

3.3 .1 Detection Algorithm ................ 32 


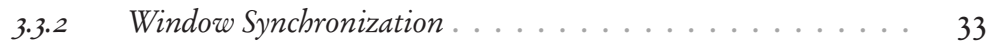

3.3.3 BER Performance and the Zero-padding Factor........ 35

xviii

3.4 Double Differential PSK ................. 36

3.4.1 The Autocorrelation Based Demodulator . . . . . . . . 36

$3.4 .2 \quad$ Synchronization for PSK . . . . . . . . . . 38

3.5 Scaling Offset Tolerance (BER and Complexity Evaluation) . . 42

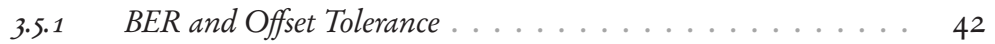

$3.5 .2 \quad$ Complexity..................... 46

3.6 Conclusion .......................... 49

4 Shifted Correlation Demodulator for DDPSK 53

4.1 Introduction . . . . . . . . . . . . . . 53

4.2 Related Work ... . . . . . . . . . . . . 54

4.3 Problem Statement ... . . . . . . . . . . . 55

4.4 The Proposed Demodulator ............... $5^{8}$

4.5 Error Probability ... . . . . . . . . . . . . . 62

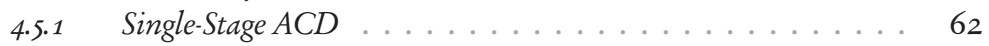

4.5 .2 Two-Stage ACD ................... 66

4.6 Simulation Results and BER Performance Analysis ... . . . 68

4.7 Complexity and Performance Trade-off ... . . . . . . 71

4.8 Conclusion ........................ 73

5 Low Complexity Implementation of THE FSK DEMOdula-

$\begin{array}{ll}\text { TOR } & 75\end{array}$

5.1 Introduction . . . . . . . . . . 75

5.2 A Brief Review of the Problem .............. 76

5.2.1 Demodulator and Synchronization Algorithm ....... 76

5.2.2 A Quick Look at Complexity .............. 78

5.3 Related Work on the Sliding DFT (SDFT) .......... 80

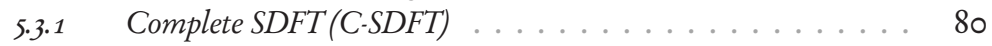

$5.3 .2 \quad$ Single Bin SDFT(SB-SDFT) . . . . . . . . . 82

$5.3 .3 \quad$ Concluding Literature. . . . . . . . . . . . 83

5.4 The Proposed Window Synchronization Algorithm ...... 84

5.4.1 Bins of Interest and the Proposed Synchronization Concept . . . 84

5.4 The Zoom Stage . . . . . . . . . . . . . . 85

5.5 The Proposed SB-SDFT with Zero-Padding . . . . . . . . . . 88

5.6 Complexity Analysis ... . . . . . . . . . . . . 91

$5.6 .1 \quad$ Complex Operations.................. 92 


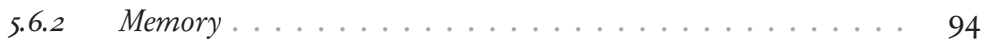

$5.6 .3 \quad$ Comparison ........................... 94

5.7 Simulation Results and Discussion . . . . . . . . . . . . 95

$5.7 .1 \quad$ Design Parameters . . . . . . . . . . . . . . . . 95

xix

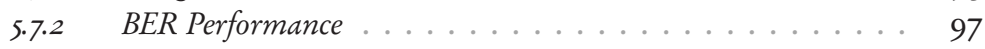

$5.7 .3 \quad$ Complexity....................... 99

5.8 Conclusion ... . . . . . . . . . . . . . . . . . 101

6 Hybrid Modulation and Time Diversity 105

6.1 Introduction . . . . . . . . . . . . . . . . 105

6.2 Related Work ... . . . . . . . . . . . . . . . . 107

6.3 Offset Tolerant Hybrid Demodulator . . . . . . . . . . . . . 108

6.3.1 Modulator Design . . . . . . . . . . . . . . 108

6.3.2 Demodulator Design .................. 109

6.3.3 Tolerable Frequency Offset ................ 114

6.4 The Proposed Demodulator in a Time-Varying Channel . . . 115

6.5 System Design for Time Diversity . . . . . . . . . . . . 121

6.6 BER Performance in AWGN Channel . . . . . . . . . . 125

6.7 BER Performance in a Time-Varying Channel . . . . . . . . . 127

6.7.1 Constant Symbol Rate Scenario . . . . . . . . . . . . . . . 129

6.7.2 Equal Bandwidth Scenario ................ 131

6.8 Conclusion . . . . . . . . . . . . . . . . . . . . 135

7 Conclusion 137

7.1 Summary . . . . . . . . . . . . . . . . . 138

7.1.1 Offset Tolerant Demodulators for UNB .............. 138

7.1.2 Scalable Offset Tolerance ................... 139

7.1.3 Offset Tolerance and Temporal Fading ............. 141

7.2 Main Contributions ... . . . . . . . . . . . . 142

$7 \cdot 3$ Recommendations for Future Work ... . . . . . . . . 143

A Mathematical Proof for $k_{c}$ Calculation Algorithm 147

A.1 Calculating the Components of $\mathscr{X}(\Omega) \ldots \ldots \ldots . . . .148$

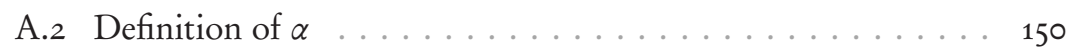

A.3 Proving $\alpha=0$ Is Maximum ... . . . . . . . . . . 152

B Complexity of Hara Synchronization with SFFT 155 
C Hybrid Demodulator in Time-Varying Channel

D Ber Curves for Hybrid Demodulator

165

D.1 BER Curves for Constant Symbol Rate Scenario 165

D.2 BER Curves for Equal Bandwidth Scenario

172

O

Acronyms

181

BibliOgRAPHY

183

List of Publications

193 


\section{1 \\ INTRODUCTION}

\subsection{Massive Connectivity}

Wireless Sensor Networks (WSN) consist of numerous small sensor nodes deployed densely in the area of interest. They might be utilized for health care, security, environmental monitoring and home automation applications $[6,8,11,104]$. Some issues must be addressed to properly take advantage of such networks most of which arise from limited resources available for sensor nodes. One of the prominent limitations regarding WSNs is the availability of energy. Either using batteries or energy harvesting methods, sensor nodes face tight energy budgets. In case of battery driven nodes, it is usually difficult or even impossible to change or recharge the batteries which demonstrates the importance of power efficiency of nodes. Moreover, WSNs have limited bandwidth resources (the ISM band is the most popular option); therefore, they should use the available bandwidth efficiently [104]. Furthermore, the utilized bandwidth might be shared with other applications such as WiFi and GSM. Consequently, sensor node receivers are exposed to a considerable level of interference which must be considered in their design.

The large number of connected sensors and personal devices such as wearables, phones, laptops ,TVs and so on has triggered the emergence of a new concept called the Internet of Things (IoT). The IoT extends the concept of internet from PCs to a variety of objects which are used in our daily lives; from a smart phone to a dimmable light at home. This provides a platform where different objects can communicate and share information as well as computing resources [7]. The new opportunities in topics such as big data analytics has emerged from this vast connectivity while now these applications are even imposing more pressure to boost the connectivity. A variety of once futuristic ideas are a reality now such as smart cities, remote sensing and monitoring, connected cars and smart industries. All together these are steering our world towards the so called fourth industrial revolution [46].

Although all applications using this massive connectivity rely on establishing 

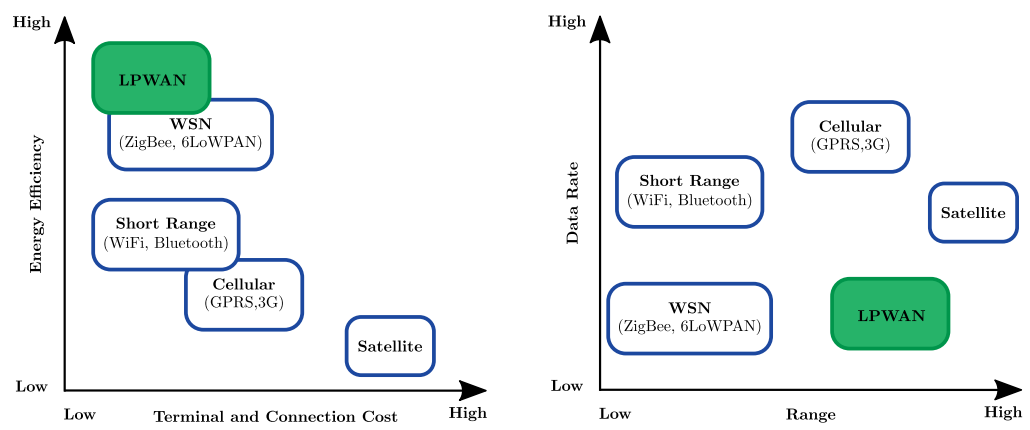

Figure 1.1: Comparison of different networking technologies [91]

a proper communication link, they each dictate different specifications for the underlying wireless communication. Range, data rate, power consumption, latency and Quality of Service (QoS) requirements of communication depend on the application. When wireless nodes are used for video streaming they need a higher bandwidth than a wireless communication node which is providing information about soil moisture. Unexpected latency in a wireless link used for connecting autonomous cars may threaten people's life while it does not harm anybody if communication with a smart meter or street lighting control system is sometimes delayed for a few seconds. Thus, we may need hundreds of $\mathrm{MHz}$ bandwidth for one application while the other is satisfied with as low as a few hundreds of $\mathrm{Hz}$. In one application we care about milliseconds whereas we can wait even a few hours for another. Some have a secure power source like a sensor connected to the car battery while others rely on scavenging ambient energy. This has encouraged different types of networks with different characteristics to handle specific applications.

\subsubsection{Low Power Wide Area Networks (LPWAN)}

A recently defined wireless network is the Low Power Wide Area Network (LPWAN) which focuses on providing a wide area coverage $(10-50 \mathrm{~km}$ in rural areas and $1-5 \mathrm{~km}$ in Urban areas) and low power communication for low data rate applications [13, 41, 62, 76, 83, 86, 95]. Smart meters, lighting control, remote sensing, precision agriculture, wild-life monitoring, parking management, some Machine-to-Machine communications in industrial zones and far more, are applications where a long rage communication and increased battery life is desired whereas, they do not impose strict latency or throughput requirements. Figure 1.1 illustrates how LPWAN is compared to other technologies with respect to data rate, energy efficiency, range and terminal connection cost [91].

Different technologies have been proposed for implementation of a LPWAN and they are still developing. There are three main classes of technologies to deploy LPWANs including narrowband technologies (e.g. Narrowband IoT (NB-IoT)), 


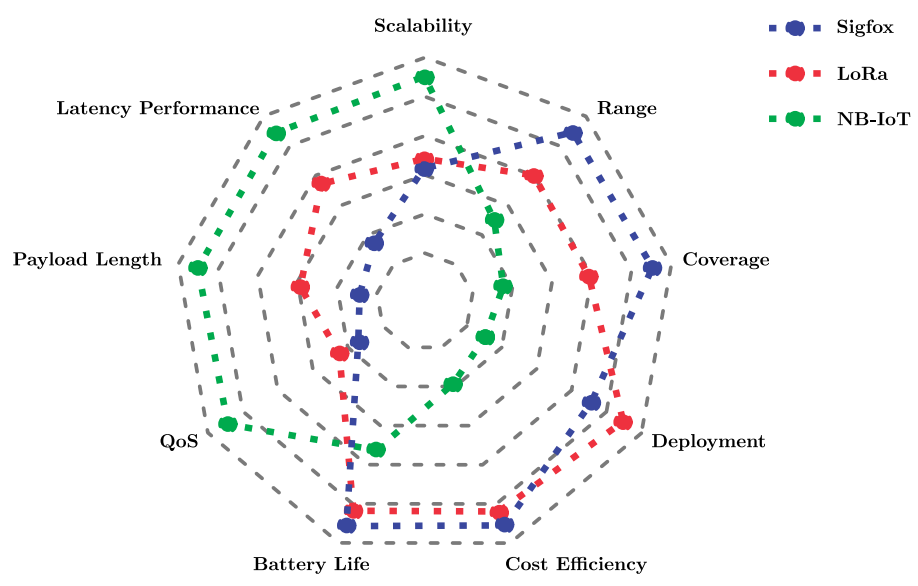

Figure 1.2: Specifications of three technologies for LPWAN [66]

spread spectrum based technologies (e.g. LoRa [1]) and Ultra-Narrowband (UNB) (e.g. Sigfox [2], Telensa [3], Wightless-N [4]) [66, 86]. Figure 1.2 shows an overview of specifications of NB-IoT, LoRa (spread spectrum) and Sigfox (UNB) which are three leading technologies for LPWANs [66].

NB-IoT provides better QoS, lower latency and larger payload transmission while its range, coverage, cost efficiency and energy consumption are limiting factors. Moreover, it operates in a licensed frequency band. Spread Spectrum based techniques have better latency performance and offer longer payloads than UNB while UNB provides longer range and wider coverage while being more energy efficient. Each technology can serve a specific purpose and the choice depends on the target application. Among these technologies, UNB communication has the advantage of reduced noise contribution due to its extremely narrow bandwidth [22]. That is why it can provide a long range communication (as long as $50 \mathrm{~km}$ in rural areas). In the following, we focus on Ultra-Narrowband communication as one of the interesting technologies in LPWAN and introduce the Slow Wireless project which aims at investigating UNB communications for interference rich environments.

\subsubsection{Ultra-Narrowband (UNB) COMMUnication}

If exploited wisely, the relaxed throughput specification of LPWAN can be leveraged to meet its low power and long range requirements which is the idea behind Ultra-Narrowband communication [25, 32, 38, 69, 70, 74, 88, 96, 107]. In many applications a limited amount of information is transferred (low throughput required) and the latency requirement is relaxed. In such cases, the instantaneous data rate requirement can be very low (in the order of $100 \mathrm{bps}$ ) even if the communication is aggressively duty cycled $[2,3,62]$. A very low data rate naturally means that it is possible to transmit with a very narrow bandwidth. This has 
encouraged applying Ultra-Narrowband communication which can be beneficial in scenarios with a large link budget such as long range communication and interference-rich environments $[62,74]$.

In LPWAN technologies a communication system using narrowband single carrier communication without spread spectrum techniques with a bandwidth in the order of a few hundreds of $\mathrm{Hz}$ is referred to as $\mathrm{UNB}$ communication $[62,66]$. There are different UNB communication technologies used for LPWAN such as Telensa [3], Wieghtless [4] and Sigfox [2].

One of the examples of a commercial UNB-based LPWAN is Sigfox [2]. With a data rate of $100 \mathrm{bps}$, it uses a channel of $192 \mathrm{kHz}$ in the Sub-GHz ISM band and uses Phase-Shift-Keying and Frequency-Shift-Keying modulation schemes. PSK and FSK are the most common modulation techniques in UNB communication technologies $[66,86]$. Similar to many other LPWANs, Sigfox uses a star topology where nodes can communicate with a base station and communication is supported for both uplink and downlink (depending on the node model and type of network). A base station supports several nodes (up to $50 \mathrm{k}$ devices per cell). The communication is performed in short packets (around 200 symbols) during packet times which are on average $2 s[2,66,83]$.

Inspired by the emerging application of UNB communication, the Slow Wireless project began to investigate the physical layer (PHY) aspects of UNB communications in the ISM band. The project is briefly described in the next section.

\subsubsection{The Slow Wireless Project}

The ISM band is a popular band for many wireless applications as it is free of charge. This makes the interference level very high and requires applications to overcome these high levels. The main idea of the Slow Wireless project is using UNB communication in an interference rich environment for low throughput networks. One of the possible applications is for the nodes which want to operate in the $2.4 \mathrm{GHz}$ ISM band which is the same band used by WiFi signals. Considering the large number of devices using WiFi for communications including smart phones and laptops, it would be challenging for low power nodes to combat this high level of interference. Moreover, it has been shown that ultranarrowband is a suitable option for energy scavenging low throughput WSNs when high levels of interference are experienced from co-existing communication systems. Particularly, when the interference is wideband compared to UNB (such as WiFi in $2.4 \mathrm{GHz}$ band) [63]. Nevertheless, a long range can also be achieved using the same UNB communication, particularly, if it works in the sub-GHz ISM band [63].

The Slow Wireless project focuses on the PHY aspects to investigate UNB communications in the ISM band. As one of the work packages in the Slow Wireless project, the current thesis aims at digital signal processing techniques for low power and low cost nodes in UNB communications. The UNB nodes are 
power constrained in applications where either the node is battery powered or uses energy scavenging techniques. Unlike other communication schemes such as Orthogonal Frequency Division Multiplexing (OFDM), narrowband communication does not require a complicated demodulator and signal processing. Furthermore, low data rate communication does not require a high sampling frequency which relaxes the requirements for processing resources in the digital circuitry. Although low data rate communication relaxes power and computational load on the digital processing part of the node's receiver, it faces two other challenges; frequency offset and temporal fading. These two challenges, as well as interesting directions to find a solution for them are explained in the next section.

\subsection{UNB Challenges}

\subsubsection{Frequency OfFSET}

One of the well-known challenges in wireless communications is frequency offset. Frequency offset may be a consequence of mismatch between local oscillators in the transmitter and the receiver or the Doppler shift due to relative movement of the receiver and the transmitter. For medium or high data rate communication, it is easier to tolerate frequency offset. For example, the Bluetooth specification allows an initial frequency offset up to $\pm 75 \mathrm{kHz}$ which is around $\pm 30 \mathrm{ppm}$ for the carrier frequency of $2.4 \mathrm{GHz}$ [47]. Considering that the data rate in Bluetooth is about $1 \mathrm{Mbps}$, this range of frequency offset is $\pm 7.5 \%$ of the data rate.

When the ratio of the data rate (signal bandwidth) to the carrier frequency decreases, the problem of frequency offset becomes more challenging. In the uplink communication of a UNB network, the base station has enough resources to afford precise frequency generation. However, the receiver in the downlink communication is a low power and cheap node which makes precise frequency generation far more challenging. In a UNB communication system with a data rate of $100 \mathrm{bps}$, achieving a frequency precision of about $\pm 7.5 \%$ of the data rate requires a precision of less than $9 \mathrm{ppb}$ (parts per billion) in the $868 \mathrm{MHz}$ band and about $3 \mathrm{ppb}$ in the $2.4 \mathrm{GHz}$ band. Even high precision crystals with $0.2-2$ ppm [81] stability lead to a frequency offset between $174-1740 \mathrm{~Hz}$ in $868 \mathrm{MHz}$ band which can reach up to more than 17 times the data rate. Such crystals for high precision frequency generation are costly and power hungry which is not suitable for cheap devices and large deployment [38].

The low cost off-the-shelf crystals have an instability in the order of 20 ppm [55]. For a UNB communication system working in the $868 \mathrm{MHz}$ band, such a precision causes a frequency offset around $\pm 17 \mathrm{kHz}$ which is 170 times the data rate. Thus, for UNB communication, frequency uncertainty is a paramount challenge. In Sigfox, this effect is alleviated using Random Frequency and Time Multiple Access in the Medium Access Control (MAC) layer. For uplink transmission, 
each node transmits a signal at a random time instant and frequency (included in the $192 \mathrm{kHz}$ band). The base station scans the whole band $(192 \mathrm{kHz})$ and detects uplink messages. Then, for a downlink message, the base station extracts the exact carrier frequency of the received signal from each node and transmits exactly on the same frequency. The effect of such a MAC layer on interference and network performance have been discussed extensively in [37, 38].

Although the frequency offset problem can be partially addressed in the MAC layer (similar to what is done for Sigfox), due to the frequency drift caused by the low cost crystals as well as long turnaround times $(30 \mathrm{~s})$, the frequency offset can still become a few times the signal bandwidth [55]. Moreover, such a solution limits the downlink transmission to the reception of an uplink message. In other words, the base station can only talk to a node a short time after the reception of an uplink message. If the receiver node can tolerate a large frequency offset, not only low cost and low power crystals can be easily used but also the strict criterion on downlink communication in UNB networks can be relaxed.

\subsubsection{Scalable Offeet Tolerance}

Generally speaking, in a wireless receiver, the frequency offset should be estimated and compensated in order for the demodulator and detector to perform correctly. To handle a large frequency offset in the receivers, there are usually two or more steps of frequency synchronization. First, a coarse estimation of the frequency offset is achieved and the frequency offset is compensated [68]. Compensation at this step might be done in the digital domain or in the analog domain by tuning the local frequency synthesizer. When the frequency offset is decreased to a fraction of the data rate, the residual frequency offset can be estimated and compensated before demodulation and detection.

The process of carrier synchronization is power hungry and costly. Therefore, offset tolerant demodulators have been proposed as an alternative to this conventional method of estimating and compensating frequency offset $[14,18,19,43$, $49,50,55,56,75,84,94,99,102,106]$. These demodulators have been designed for both Phase Shift Keying (PSK) and Frequency Shift Keying (FSK) modulations. Various offset tolerant demodulators for FSK have been proposed for Bluetooth receivers as well as Wireless Sensor Networks (WSN) applications $[14,18,43,56,102]$. A class of demodulators based on Double Differential PSK (DDPSK) have also been proposed which are capable of tolerating frequency offset $[49,84,94,99,106]$. In most of these proposed methods, the allowed offset tolerance is limited and it is assumed that the frequency offset is compensated so that it is brought in the range of the data rate using a coarse carrier synchronization. Afterwards, using an offset tolerant demodulator, the fine carrier synchronization is avoided to eliminate precise synchronization algorithms. As explained in the previous section, for UNB communication, even a frequency offset as large as the data rate is significantly small. Thus, it would be interesting 
to apply demodulation schemes with a scalable offset tolerance capability. In this way, we can remove (or simplify) the coarse carrier synchronization as well.

Among the proposed demodulators, there are two general architectures that have potential for high scalability $[39,44,94,106]$. The main idea of these demodulators is using the Discrete Fourier Transform (DFT) for FSK demodulation $[39,44]$ and Double Differential detection for PSK demodulation [106]. These digital demodulators have been originally designed for low data rate satellite communications where the frequency offset caused by Doppler shift can be much larger than the data rate $[44,106]$. These demodulators can theoretically tolerate any frequency offset as far as the signal is still captured by the input filter. Although these demodulators are in principle scalable, they face limitations when it comes to very large frequency offset (like $1.7-17 \mathrm{kHz}$ for a $100 \mathrm{bps}$ data rate which was mentioned earlier). Nevertheless, these structures can be modified to design offset tolerant demodulators for UNB communications.

\subsubsection{Temporal Fading and Offset Tolerant Demodulators}

In addition to frequency offset, temporal fading should be considered in UNB communication as well. As shown in [71], for low data rate communication a time-varying channel may cause an error floor. This error floor is more drastic in ultra-narrowband systems due to a longer symbol time. As a consequence of time-varying behavior, channel estimation at the beginning of a packet is not reliable. Furthermore, using multiple pilot sequences for channel estimation imposes prohibitive overhead due to short packets used in these systems (as short as 200 symbols [38]). Offset tolerant demodulators proposed for FSK and PSK do not need channel estimation to overcome the phase offset resulting from a fading channel. Nevertheless, the attenuation of the signal in presence of fading as well as random frequency modulation caused by a frequency-dispersive channel degrades the performance terribly and may lead to an error floor.

In such circumstances diversity techniques can be utilized to improve performance [71]. Space diversity is dismissed for wireless sensor nodes because of low power and area constraints of the receiver. In a time-varying channel, time diversity (using channel coding and interleaving) is a possible solution. Additionally, frequency diversity (using frequency hopping) can be utilized. Both solutions involve transmission of redundant information via channel coding. This increases either the bit rate or packet time. To keep the packet time constant (avoiding longer on-time for the RF front-end) while keeping the symbol rate low, higher order modulation can be used and the offset tolerant demodulators can be applied.

For a PSK modulation, the downside of the increased modulation order is a loss in BER performance. On the other hand, increasing the FSK order increases the required bandwidth. To achieve a better power and bandwidth trade-off in higher order modulation, hybrid frequency/phase modulation has been suggested [17, $34,35,52,57,87]$. Despite all research performed on different aspects of such a 
hybrid modulation, an offset tolerant demodulator, which is capable of tolerating frequency offset larger than the symbol rate, has not been introduced. If an offset tolerant demodulator is designed for hybrid modulation, it can increase the raw bit-rate. Then, this additional raw bit-rate can be used to implement a low complexity diversity technique and improve the performance of UNB communication in a time-varying fading channel.

\subsection{Research Objectives}

So far, offset tolerant demodulators have been introduced as a method which has proven useful to overcome the challenge of frequency offset. In many applications the offset tolerant demodulators are used to remove the requirement of the fine tune offset tolerance. In UNB communication this fine-tuned frequency offset, which is a fraction of the signal bandwidth, can be in the order of $10-20 \mathrm{~Hz}$ for a $868 \mathrm{MHz} / 2.4 \mathrm{GHz}$ carrier. This means that it already needs considerable coarse carrier recovery before the fine tuning stage.

As mentioned earlier, some protocols like Sigfox, try to solve the offset problem at a higher layer. However, it still imposes limitations on the communication and, when low cost and low power crystals are used, it still leads to frequency offset larger than the signal bandwidth [55]. Considering these facts, an offset tolerant demodulator which is able to tolerate arbitrarily large frequency offset can overcome the offset problem and add to the flexibility of the design of UNB systems and networks. It eliminates the need for costly precise crystal or power hungry thermal compensation and removes carrier recovery loops or considerably relaxes their requirements.

The techniques proposed in this work focus on the baseband digital processing and are independent of the analog design. Therefore, these techniques are applicable to different receiver architectures. As an example, a low-IF receiver, which is a popular structure for low power wireless receivers, is shown in Figure $1.3[55,64]$. The signal frequency is down-converted to a low Intermediate Frequency (IF) $(1-5 \mathrm{MHz})$, converted to digital samples using an Analog to Digital Converter (ADC) and sent to the digital domain. The down-conversion to baseband, filtering and decimation and, finally, demodulation and detection are performed in the digital domain. In UNB communications, the signal bandwidth is very small compared to the IF frequency (in the order of $10^{-4}$ of IF) so the analog section e.g. the analog low-pass filter before the ADC will not have a significant effect on the demodulator offset tolerance.

Therefore, a digital offset tolerant demodulator that can work independent of the $\mathrm{RF}$ front-end can provide flexibility and move the problem and its solution to the digital domain which can be more power efficient. Besides, the narrowband filters required to deal with the UNB signal can be implemented more efficiently in the digital domain. To achieve a digital demodulator while removing precise carrier recovery in UNB communications, this thesis focuses on demodulators 


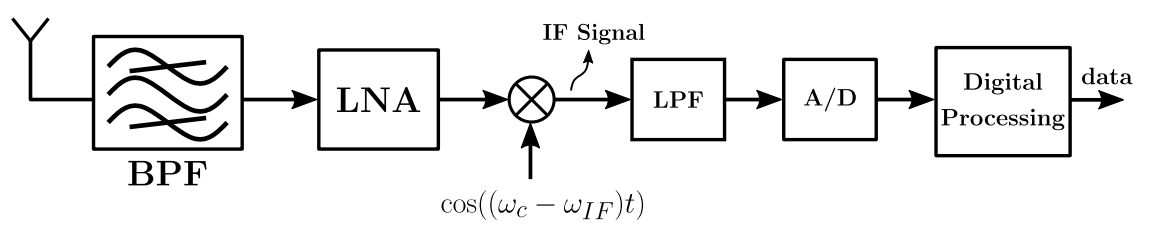

Figure 1.3: A low-IF receiver architecture for wireless communications

which can achieve scalable offset tolerance. Alleviating the frequency offset challenge within the PHY layer offers flexibility to higher layers and network designers. In addition to the frequency offset, the temporal fading and using diversity as a solution should be investigated. Taking all aforementioned into account, the main research questions can be formulated as follows.

1. What are the limitations of offset tolerant demodulators, which can tolerate large frequency offset, for UNB communications?

To answer this question a frequency offset tolerant demodulator for FSK is introduced inspired by an existing DFT-based demodulator. An offset tolerant demodulator for PSK is also considered. The two demodulators are simulated for different offset tolerance ranges and limitations of these demodulators for scalable offset tolerance are investigated.

2. How can the limitations of the demodulators to achieve scalable offsettolerance be circumvented?

When the limitations of demodulators for FSK and PSK are known, we need to resolve them. It will be shown that the limitation for the offset tolerant PSK demodulator is Bit Error Rate (BER) performance degradation as a result of the increased noise bandwidth. In case of the DFT-based demodulator for FSK, the complexity will be prohibitive when a large offset tolerance is required. To overcome these challenges, two different algorithms are proposed for PSK and FSK offset tolerant demodulators.

3. How can the temporal fading in UNB communication be counteracted and the required redundancy for diversity be achieved while using offset tolerant demodulators?

To combat the fading effect in a time-varying channel, time diversity might be used and it is a particularly interesting solution as it does not add any overhead unlike frequency hopping. Nevertheless, to achieve redundancy used for coding we need to increase the raw bit-rate. As mentioned earlier, a hybrid frequen$\mathrm{cy} /$ phase modulation can accomplish this task in a more power efficient manner than higher order PSK and more bandwidth efficient way than higher order FSK. Thus, an offset tolerant demodulator for hybrid FSK/PSK modulation is proposed and its performance in a time-varying channel and using time diversity is evaluated. 


\subsection{Outline}

In the next chapter, an overview of FSK and PSK demodulation and their demodulators is presented. Chapter 3 which is based on [S:3] includes the answer to the first question. A DFT-based demodulator for FSK is introduced and is compared with an offset tolerant demodulator for PSK. This chapter elaborates on how they perform in the presence of a large frequency offset and what the limitations of each method are. Then, the next two chapters are dedicated to finding a solution to address the limitations observed in Chapter 3 and answer the second question. To address the BER performance loss of a PSK offset tolerant demodulator in the presence of a large frequency offset, a shifted correlation demodulator is proposed in Chapter 4 which is based on [S:4]. In Chapter 5, a low complexity synchronization algorithm for the DFT-based demodulator and its implementation are presented to relax the complexity bottleneck of the FSK offset tolerant demodulator [S:2, 6]. In the next step, Chapter 6 focuses on the third question. Based on [S:1, 5], this chapter presents an offset tolerant demodulator for hybrid frequency/phase modulation. The demodulator is used for incorporating time diversity in a time-varying channel and its performance is evaluated for different scenarios. Finally, in Chapter 7 conclusions are drawn and recommendations for future work are presented. 


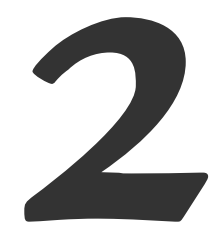

\section{BACKGROUND}

Before continuing our path to offset tolerant demodulators for UNB communication we need to first have a brief review on the concepts used in the thesis and the conventional modulation and demodulation schemes. The focus of our work is on demodulating the received signals in the presence of a frequency offset; therefore, we mainly discuss the receiver of a wireless communication link. Besides, as mentioned in the previous chapter, the reception of the uplink signal in UNB communication is less challenging as the base station (gateway) does not have critical energy restrictions. Thus, the main focus is on the receiver design for the downlink communication. In this chapter, first a simple model of the wireless receiver and the complex notation of signals are presented. Then, the modulation schemes together with their demodulators are presented. Afterwards, the problem of frequency offset is explained and the concept of a receiver with an offset tolerant demodulator is demonstrated.

In the next section, the system model and signal notations are defined. Sections 2.2, 2.3 and 2.4 introduce FSK, PSK and FPSK modulations and some of their typical demodulators. Finally, section 2.5 elaborates on the frequency offset problem and shows how it is solved in a conventional way and how we are going to address it in this work.

\subsection{System Model and Complex Notations}

A wireless signal is transmitted using a sinusoidal carrier with a frequency equal to $f_{c}$. When information bits are transmitted modulating the frequency or phase, the general form of the transmitted signal during the $n^{\text {th }}$ symbol is as follows.

$$
s(t)=A_{c} \cos \left(\omega_{c} t+\theta(t)\right),(n-1) T \leq t \leq n T
$$

where $A_{c}$ is the signal amplitude, $T$ is the symbol period, $\omega_{c}=2 \pi f_{c}$ is the carrier frequency and $\theta(t)$ is the phase of the signal which reflects modulated information either on frequency or phase. In (2.1) a rectangular pulse shape is 


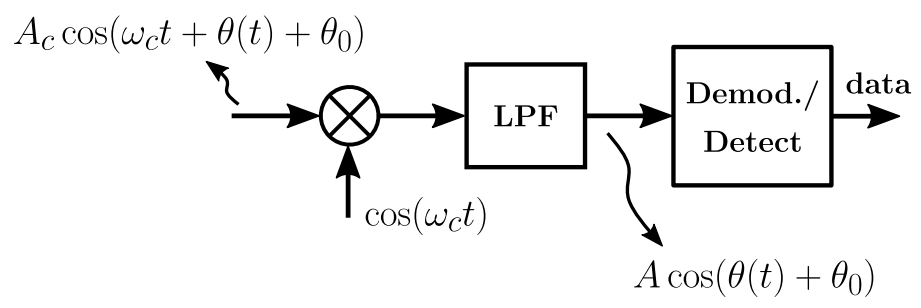

Figure 2.1: A simplified model of a wireless receiver

assumed. At the receiver, from antenna to the data bits, several analog and digital blocks are used to process the received signal and detect the information; however, we do not intend to go into the details of these blocks. A very simplified model of a wireless receiver which receives a phase or frequency modulated signal is shown in Figure 2.1.

At the receiver, the carrier frequency is removed by mixing the received signal $\left(A_{c} \cos \left(\omega_{c} t+\theta(t)+\theta_{0}\right)\right)$ with a locally generated replica of the carrier and low-pass filtering. The downconverted signal $A \cos \left(\theta(t)+\theta_{0}\right)$ (where $A$ is the scaled amplitude after filtering) is sent to demodulator. $\theta_{0}$ in the received signal represents a constant phase offset which is the result of imprecise carrier phase recovery at the receiver. Then, the baseband signal $\left(A \cos \left(\theta(t)+\theta_{0}\right)\right)$ is demodulated and transmitted data is extracted. In some receiver architectures, the signal is first converted to a non-zero frequency called Intermediate Frequency (IF) which is much lower than the carrier. Then, the IF signal may be either used immediately to demodulate and detect the signal or downconverted to baseband before demodulation.

For convenience in mathematical notations, the real valued bandpass signals at $\mathrm{RF}$ are demonstrated using a canonical representation [45]:

$$
s(t)=\operatorname{Re}\left[\tilde{s}(t) \times e^{j \omega_{c} t}\right]
$$

where $\operatorname{Re}[$.$] shows the real part of the signal. \tilde{s}(t)$ is called the complex envelope of the signal and is defined as follows.

$$
\tilde{s}(t)=A_{c} e^{j\left(\theta(t)+\theta_{0}\right)}=s_{I}+j s_{Q}
$$

From (2.2) and (2.3) it can be seen that $s(t)=s_{I} \cos \left(\omega_{c} t\right)-s_{Q} \sin \left(\omega_{c} t\right)$. Considering trigonometric properties, $s_{I}$ and $s_{Q}$ can be achieved by multiplying the received signal by $\cos \left(\omega_{c} t\right)$ and $-\sin \left(\omega_{c} t\right)$ and selecting the zero frequency component by a low-pass filter. $s_{I}$ and $s_{Q}$ are called the in-phase and quadraturephase (or simply quadrature) components of the signal, respectively. Both of these components might be used for signal processing in the receiver. 
Using the complex envelope notation and assuming an Additive White Gaussian Noise (AWGN) channel, the received baseband signal is:

$$
\tilde{r}(t)=A_{c} e^{j\left(\theta(t)+\theta_{0}\right)}+n(t)
$$

where $\tilde{r}(t)$ is the baseband signal and $n(t)$ is complex Gaussian noise $(n(t)$ is not shown in Figure 2.1). In most practical cases, the exact phase recovery of the carrier is cumbersome. That is why such a phase offset is considered and demodulators are designed to perform independent of phase offset. The demodulators which do not rely on carrier phase recovery for correct performance are called non-coherent demodulators.

\subsection{FSK MOdulation}

FSK modulation transmits information by changing the frequency of the transmitted carrier. For Binary FSK (BFSK) the complex envelope of the modulated signal during the $n^{\text {th }}$ symbol period is as follows.

$$
\tilde{s}(t)=A_{c} e^{j b_{n} \frac{h \pi}{T} t},(n-1) T \leq t \leq n T
$$

where $b_{n}=-1,+1$ is the transmitted symbol and $T$ is the symbol period. $b$ is called modulation index which determines the separation between two possible tones of a BFSK signal which is called frequency separation defined as $\omega_{\text {sep }}=$ $2 \pi h / T$.

A more practical version of an FSK signal is called Continuous Phase FSK (CPFSK). The phase of a continuous phase binary FSK is derived as follows [103].

$$
\theta(t)=\frac{\pi h}{T} b_{n}(t-(n-1) T)+\pi h \sum_{i=0}^{n-2} b_{i},(n-1) T \leq t \leq n T
$$

Using CPFSK, the received signal during the $n^{\text {th }}$ symbol is as follows.

$$
\tilde{r}(t)=A_{c} e^{j\left(\theta(t)+\theta_{0}\right)}+n(t),(n-1) T \leq t<n T
$$

where $\theta(t)$ is obtained from (2.6), $\theta_{0}$ is a constant phase offset and $n(t)$ represents complex Gaussian noise. The FSK modulated signal can be demodulated and detected using a non-coherent demodulator for orthogonal modulations as shown in Figure 2.2 [45].

In each branch, the signal is multiplied by the conjugate of one of the possible transmitted frequencies and, then, integrated over a symbol period. Afterwards, 


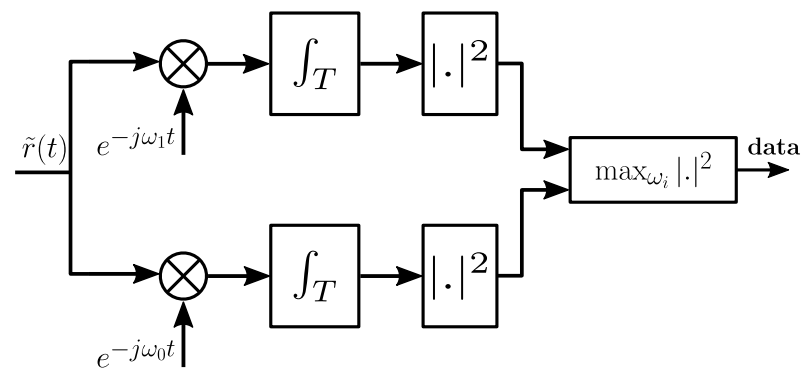

Figure 2.2: Non-coherent demodulator for BFSK based on correlation

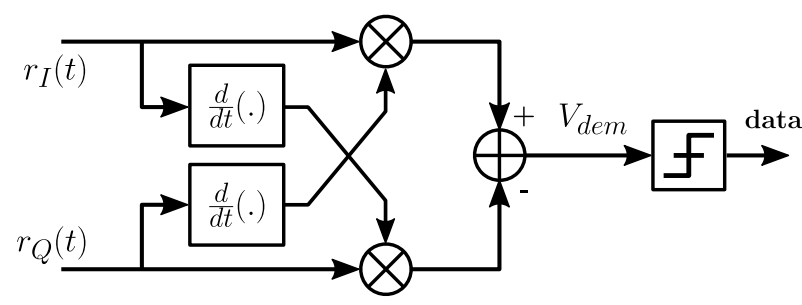

Figure 2.3: Cross Differentiate Multiply demodulator for BFSK

the magnitudes of the integrated outputs are calculated and compared and the maximum determines which signal was originally transmitted.

The two parts shown by a dashed box in each branch form filters which are matched to each possible transmitted signal. The multiplication and integration in Figure 2.2 is actually a correlation of the signal with either of the possible transmitted symbols; thus, this architecture is also called correlation based demodulator [45]. In this demodulator, the magnitude of the integrator output degrades drastically in the presence of frequency offset; thus, frequency offset must be precisely estimated and compensated so that this demodulator can be used.

Another type of demodulator for FSK is the Cross Differentiate Multiply (CDM) demodulator. This demodulator can be implemented in both the digital and the analog domains and its basic principle is shown in Figure 2.3 [56]. To clarify the idea, let us consider a simple case where the baseband signal is $e^{ \pm j \frac{\omega_{s e p}}{2} t}$. In this case, the in-phase and quadrature components are $\cos \left( \pm \frac{\omega_{\text {sep }}}{2} t\right)$ and $\sin \left( \pm \frac{\omega_{\text {sep }}}{2} t\right)$, respectively. The derivative of these components can be obtained as follows.

$$
\begin{aligned}
& \frac{d}{d t}\left(\cos \left( \pm \frac{\omega_{\text {sep }}}{2} t\right)\right)=\mp \frac{\omega_{\text {sep }}}{2} \sin \left( \pm \frac{\omega_{\text {sep }}}{2} t\right) \\
& \frac{d}{d t}\left(\sin \left( \pm \frac{\omega_{\text {sep }}}{2} t\right)\right)= \pm \frac{\omega_{\text {sep }}}{2} \cos \left( \pm \frac{\omega_{\text {sep }}}{2} t\right)
\end{aligned}
$$




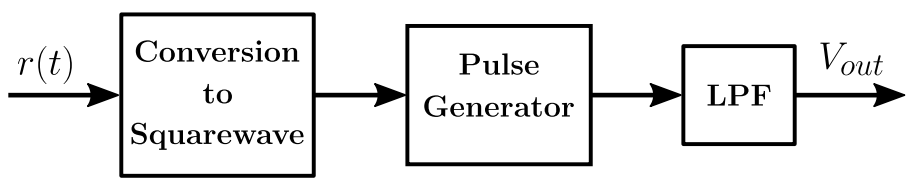

Figure 2.4: FSK demodulator based on converting frequency modulated signal to squarewave (e.g. using limiter)

Thus, the result of multiplications in the upper and the lower branch shown by $V_{I}$ and $V_{Q}$, respectively, are as follows.

$$
\begin{aligned}
V_{I} & = \pm \frac{\omega_{\text {sep }}}{2} \cos ^{2}\left( \pm \frac{\omega_{\text {sep }}}{2} t\right) \\
V_{Q} & =\mp \frac{\omega_{\text {sep }}}{2} \sin ^{2}\left( \pm \frac{\omega_{\text {sep }}}{2} 2 t\right)
\end{aligned}
$$

Finally, subtracting $V_{Q}$ from $V_{I}$, the output before the decision block is as follows.

$$
V_{d e m}= \pm \frac{\omega_{\text {sep }}}{2}\left[\cos ^{2}\left( \pm \frac{\omega_{\text {sep }}}{2} t\right)+\sin ^{2}\left( \pm \frac{\omega_{\text {sep }}}{2} t\right)\right]
$$

Since the sum of the terms in paranthesis is always equal to one, the sign of $V_{d e m}$ only depends on the sign of modulated frequency. It is clear that the output of such a demodulator will be affected by a non-zero frequency offset and the transmitted data cannot be retrieved correctly.

Another class of FSK demodulators is based on generating a square wave from the received frequency modulated signal and calculating the frequency according to transitions $[43,102,105]$. A general block diagram for this type of demodulators is shown in Figure 2.4. The square wave might be generated using a limiter which amplifies the input sinusoidal signal to saturation level or applying phase to digital converter methods such as phase quantization [43]. In a digital implementation the zero-crossings of this square wave can be counted and the frequency (and thus the transmitted data) is detected based on the number of crossing per symbol period (called a zero-crossing demodulator).

Alternatively, the square wave can be sent to a pulse generator to generate equal width pulses at each edge. Since the density of these equal width pulses changes with frequency, averaging them using a low-pass filter can convert the frequency information to a variable voltage which will be used for data detection. In this type of demodulators, frequency offset changes the intensity of zero-crossings from the expected values and adds a DC-level to the output of the demodulators and degrades detection performance. 
In some FSK demodulators, a delayed or phase shifted version of input IF (Intermediate Frequency) signal is generated and used for demodulation [14, 19, 50, 92]. FSK demodulation using a Delay Locked Loop (DLL) is an example of this type of demodulators. After converting IF FSK signal to a square wave, it is sampled by a delayed version of itself with a delay, generated by a DLL exactly equal to the period of the IF frequency. Depending on whether the received signal has a higher or lower frequency than the expected IF frequency, the rising edges of the delayed version lags or leads the rising edges of the signal itself. This characteristic is used to detect the data sequence [14]. In a DLL based demodulator, frequency offset means that the generated delay is no longer equal to the period of the IF input signal which destroys performance of the demodulator [19].

As mentioned above, the BER performance of all of these demodulators degrades in the presence of frequency offset. None of the above demodulators are offset tolerant in nature and they either need a frequency controller or some additional techniques to improve their robustness against frequency offset.

\subsection{PSK Modulation}

Phase Shift Keying (PSK) is a digital modulation technique which transmits information by changing the phase of the transmitted signal. The complex envelope of an M-ary PSK signal is:

$$
\tilde{s}(t)=\sum_{n} g(t-n T) e^{j\left(\varphi_{n}+\theta_{0}\right)}
$$

where $\varphi_{n}=\frac{2 \pi i}{M}(i=0, \ldots, M-1)$ is determined by the transmitted symbol and $\theta_{0}$ is a constant phase offset which is due to imprecise carrier phase recovery. $g(t)$ is the pulse shape used for PSK to limit the bandwidth of the transmitted signal in band-limited scenarios. In this work, a rectangular pulse shape is considered; thus, the received MPSK signal during the $n^{t h}$ symbol period is:

$$
\tilde{r}(t)=A_{c} e^{j\left(\varphi_{n}+\theta_{0}\right)}+n(t),(n-1) T \leq t \leq n T
$$

Detecting each symbol stand-alone requires prior knowledge of $\theta_{0}$ which is complex to achieve. To enable non-coherent detection, a variation of PSK modulation is used known as Differential PSK (DPSK).

\subsubsection{Differential PSK (DPSK)}

For DPSK the phase of each symbol will be used as a reference to determine the phase of the next symbol. If $\theta_{0}$ is constant during two consecutive symbols, its effect will be removed. To make the detection possible in the receiver, a differential encoder in the transmitter is used which encodes information in the 


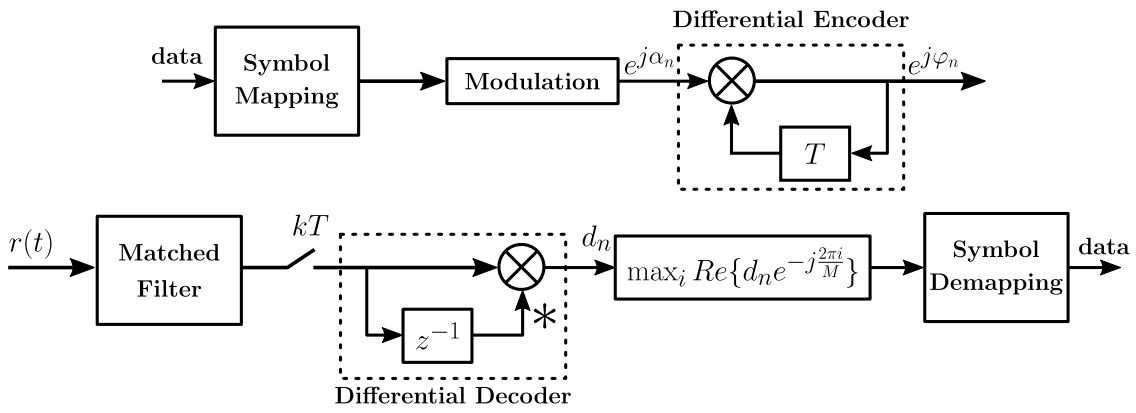

Figure 2.5: Differential encoder and DPSK demodulator

difference between the phases of consecutive symbols. The differential encoder and the DPSK demodulator for M-ary PSK modulation are shown in Figure 2.5.

In the transmitter, data is mapped to the symbols of MPSK to generate $e^{j \alpha_{n}}$. Afterwards, a differential encoder is applied to achieve $e^{j \varphi_{n}}$ which is the transmitted phase. In the demodulator, the signal passes through a Matched Filter (MF) to maximize the Signal to Noise Ratio (SNR). When a rectangular pulse shape is assumed, this filter would be as simple as integration over a symbol period. The output of the filter is sampled at the symbol rate and the values are sent to a differential decoder. The differential decoder multiplies the output of the integrator for each symbol with the conjugate of integrator output of the previous symbol. Using the differential decoder and considering a signal model similar to (2.14), the decision variable $d_{n}$ after decoder is:

$$
d_{n}=A e^{j \Delta \varphi_{n}}+n^{\prime}
$$

where $A=\left(A_{c} T\right)^{2}, \Delta \varphi_{n}=\varphi_{n}-\varphi_{n-1}$ and $n^{\prime}$ represents all noise components. Now, looking into the differential encoder, we can see that $\alpha_{n}=\varphi_{n}-\varphi_{n-1}$. Therefore, the transmitted information can be detected based on $d_{n}$ using the following detection criterion for MPSK.

$$
\hat{\alpha_{n}}=\underset{\alpha_{i} \in A}{\arg \max } \operatorname{Re}\left[d_{n} \times \exp \left(-j \alpha_{i}\right)\right]
$$

where $A=\left\{\frac{2 \pi i}{M} \mid i=0, \ldots, M-1\right\}$ is the set of possible symbol phases for an MPSK modulation.

As shown here, to detect a DPSK signal a noisy reference (the phase of the previous symbol) is used which increases the error probability compared to coherent detection. A variety of methods have been introduced in literature to overcome this BER performance loss of DPSK. In [21], a multiple symbol detection method is proposed for DMPSK. In this method a decision variable is 
derived based on observing a sequence of $N$ symbols by which $N-1$ symbols can be detected. When the number of symbols in the observed sequence increases, the BER performance approaches the BER performance of coherent MPSK. In $[58,60]$, the feedback of the previously detected symbols is used to detect future symbols. Although such methods can improve the performance, they considerably increase the complexity of the demodulator. For Binary DPSK (DBPSK) the BER performance loss is almost $1 \mathrm{~dB}$ at $B E R=0.1 \%$ while it increases for higher modulation orders (M-ary DPSK for $M>2$ ). Thus, these methods mainly target high orders of PSK.

Although DPSK is robust against phase offset it is susceptible to performance loss in presence of frequency offset. Frequency offset leads to an unknown variation of phase between two consecutive symbols; therefore, the phase of one symbol cannot be used as a reference to detect the next one. This degrades the performance of the DPSK demodulator.

\subsection{Hybrid Modulation}

It is well-known that higher order PSK, M-ary PSK (MPSK), can achieve better bandwidth efficiency while sacrificing energy efficiency. In contrast, higher order FSK, L-ary FSK (LFSK), compromises bandwidth efficiency to save energy. Hybrid frequency and phase modulation has been investigated as an alternative approach to this trade-off. The literature on hybrid modulation schemes can be classified into two groups; Quadrature Frequency/Phase Modulation (QFPM) and Frequency/Phase Shift Keying (FPSK) which is also simply referred to as frequency/phase modulation in this work. The main idea of both is simultaneous modulation of frequency and phase; however, each of them approach the power and bandwidth efficiency trade-off from a different perspective.

QFPM focuses on increasing bandwidth efficiency with lower energy loss compared to MPSK. In other words, QFPM adds quadrature frequency modulation to PSK to increase the bandwidth efficiency and it may lead to energy loss; nevertheless, the energy loss is still less compared to using only higher order MPSK for achieving the same bandwidth efficiency. In FPSK, however, frequency modulation is added to PSK to improve energy efficiency while the result will be more bandwidth efficient than using only higher order FSK. QFPM and FPSK modulation schemes are explained in the following.

\subsubsection{Quadrature Frequency/Phase Modulation (QFPM)}

A special case of hybrid modulation which focuses on increasing bandwidth efficiency with lower energy loss compared to MPSK is Quadrature/Quadrature Phase Shift Keying (Q2PSK) proposed in [90]. Similar to Quadrature PSK (QPSK) this modulation utilizes in-phase and quadrature components of the carrier; $\cos \left(\omega_{c} t\right)$ and $\sin \left(\omega_{c} t\right)$. Combining quadrature components of the car- 
rier with two sinusoidal pulse shapes $(\cos (\pi t / 2 T)$ and $\sin (\pi t / 2 T)$ where the bit rate is $\left.T_{b}=2 / T\right)$, the following four signals are derived [90].

$$
\begin{aligned}
& s_{1}(t)=\cos (\pi t / 2 T) \cos \left(\omega_{c} t\right) \\
& s_{2}(t)=\sin (\pi t / 2 T) \cos \left(\omega_{c} t\right) \\
& s_{3}(t)=\cos (\pi t / 2 T) \sin \left(\omega_{c} t\right) \\
& s_{4}(t)=\sin (\pi t / 2 T) \sin \left(\omega_{c} t\right)
\end{aligned}
$$

Binary data $(-1$ or +1$)$ is transmitted with each signal and the sum of these four can generate 16 different symbols. $\mathrm{Q}^{2} \mathrm{PSK}$ is twice as bandwidth efficient as QPSK while a coherent optimum receiver for $\mathrm{Q}^{2} \mathrm{PSK}$ can perform similar to QPSK [90]. To provide the same bandwidth efficiency with higher order PSK, a 16PSK modulation must be used which needs around $7 \mathrm{~dB}$ larger $E_{b} / N_{0}$ than $\mathrm{Q}^{2} \mathrm{PSK}$ at $B E R=0.1 \%$.

For a more practical scenario where carrier phase estimation is not feasible, a differentially coherent receiver for $\mathrm{Q}^{2} \mathrm{PSK}$ is proposed in [27]. Although coherent demodulators of $\mathrm{Q}^{2} \mathrm{PSK}$ and QPSK have similar BER performance, the non-coherent demodulator of $\mathrm{Q}^{2} \mathrm{PSK}$ requires $1 \mathrm{~dB}$ more $E_{b} / N_{0}$ than differential QPSK at $B E R=0.1 \%$. Thus, the bandwidth efficiency is achieved and power efficiency is sacrificed but still the power loss is lower compared to using only higher order PSK.

The concept of using quadrature components of the carrier was further extended to $N$ frequency tones in [33]. It uses quadrature components of $N$ different frequency tones in combination with BPSK resulting in a quadrature frequency/phase modulation with $N$ different frequencies, NQFPM. The differentially coherent detection of this generalized quadrature modulation is proposed in [16]. One of the problems associated with the quadrature frequency/phase modulation is its non-constant envelope which has encouraged researchers to suggest special coding techniques to avoid envelope variation at the expense of decreased bandwidth efficiency $[15,90]$.

In low data rate applications, highly bandwidth efficient modulation is not the primary concern; therefore, FPSK modulation which focuses on energy efficiency and has a constant envelope is a better choice than QFPM. The next subsection explains FPSK modulation.

\subsubsection{Frequency/Phase Shift Keying (FPSK)}

The main difference between FPSK and QFPM is that both in-phase and quadrature components of the carrier are modulated with the same frequency. Using FPSK decreases bandwidth efficiency but improves BER performance compared 


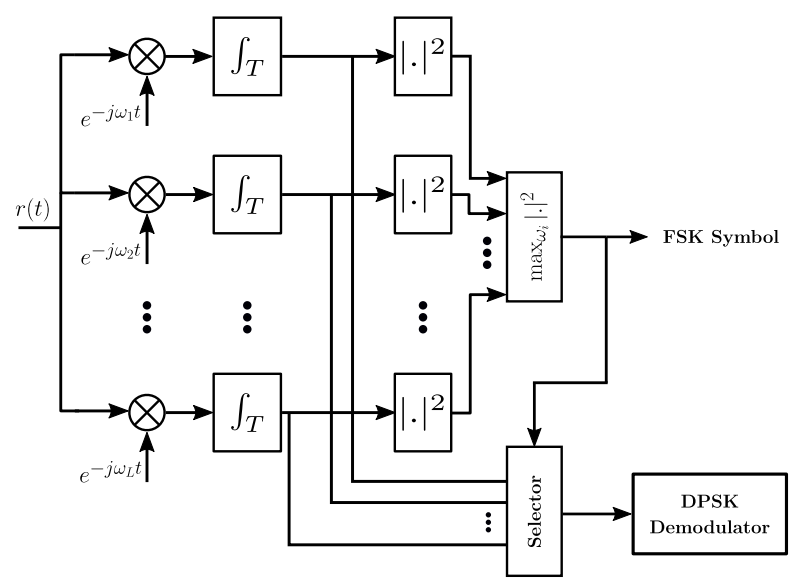

Figure 2.6: A non-coherent demodulator for FPSK.

to QFPM. One of the early examples of FPSK was proposed in [87]. The complex envelop of the modulated signal is as follows.

$$
\tilde{s}(t)=A_{c} e^{j\left(\omega_{n} t+\varphi_{n}\right)},(n-1) T \leq t<n T
$$

where $\omega_{n}=m_{n} \frac{\omega_{\text {sep }}}{2}$ and $m_{n}=2 i-(L+1)($ for $i=1, \ldots, L)$ determines the index of the symbol for FSK. $\varphi_{n}$ is the modulated phase during the $n^{t h}$ symbol. To demodulate such a signal, a combination of FSK and PSK demodulators must be used.

The non-coherent demodulator for FPSK which is a combination of FSK and DPSK is shown in Figure 2.6 [34]. First, FSK is demodulated using non-coherent demodulation. Afterwards, the result of FSK detection is used to determine the correct branch which must be sent to the next stage which is a DPSK demodulator. Similar to previous demodulators introduced in this chapter, the performance of the demodulator in Figure 2.6 depends on generating an exact replica of the carrier frequency at the receiver.

\subsection{Frequency Offeset; Compensate or Tolerate}

The mentioned demodulators need an exact replica of the received carrier frequency in the receiver. However, this may not be available at the receiver due to the mismatch between oscillators in the transmitter and the receiver or the Doppler shift between the transceiver nodes. In the presence of a frequency offset, the baseband received signal during the $n^{\text {th }}$ symbol is as follows.

$$
\tilde{r}(t)=A_{c} e^{j\left(\omega_{0} t+\theta(t)+\theta_{0}\right)}+n(t),(n-1) T \leq t \leq n T
$$


where $\omega_{O}$ is the frequency offset, $\theta(t)$ represents the effect of frequency or phase modulation, $\theta_{0}$ is a constant phase offset and $n(t)$ denotes Guassian noise. To clarify the effect of frequency offset, let us assume a structure similar to the DPSK demodulator in Figure 2.5. For a rectangular pulse shape, the filter block will be simply an integration over a symbol period. Assuming a signal model similar to (2.19), the output of the integrator will be as follows [84].

$$
\int_{0}^{T} \tilde{r}(t) d t=A_{c} T \frac{\sin (\psi)}{\psi} e^{j\left(\psi+\varphi_{n}+\theta_{0}\right)}+n^{\prime}
$$

where $\psi=\omega_{O} T / 2, \varphi_{n}$ is the modulated phase during the symbol period and $n^{\prime}$ is the integral of the noise process. In case of a correlation based detector for FSK, the correlation of the signal with the filter matched to the transmitted frequency has a similar form with a non-zero frequency offset.

According to (2.20), the effect of frequency offset is two-fold. The amplitude of the output is similar to a sinc function $\left(\frac{\sin (\psi)}{\psi}\right)$. When the frequency offset is zero, the amplitude has a maximum value of one; whereas, the amplitude decays when the frequency offset increases which leads to decreased SNR. In other words, when there is a frequency offset, the integrator is not a matched filter anymore. If the frequency offset is too large, the signal spectrum is pushed outside this filter (i.e. integrator) and the signal power at the output of the filter decreases drastically. Another effect of the frequency offset is the phase term $\psi=\omega_{O} T / 2$ in (2.20). This additional phase offset increases the error probability to detect the correct phase of the transmitted symbol.

\subsubsection{Compensating Frequency Offset}

When a demodulator similar to the aforementioned examples is used, carrier frequency synchronization must be performed. Frequency estimation algorithms can be utilized to detect the frequency offset and compensate for it. There are different digital synchronization techniques which can achieve precise frequency estimation after the matched filter $[67,68]$; however, they can only estimate frequency offsets which are a fraction of the symbol rate.

To compensate for a large frequency offset, usually a two (or more)-step carrier frequency recovery is used. The algorithms need to estimate the offset and compensate it via a feedback or feedforward structure. These are shown by estimation and compenstation blocks in Figure 2.7 [68]. First, a higher oversampling is used to perform a coarse carrier estimation and compensation. This step limits the range of the frequency offset and sends the signal for filtering and decimation. At each step, the filter bandwidth becomes narrower, sampling frequency decreases and the residual frequency offset becomes smaller. Before final demodulation, fine-tuned carrier recovery is used using the samples after the matched filter to estimate frequency with the highest precision. This is shown 


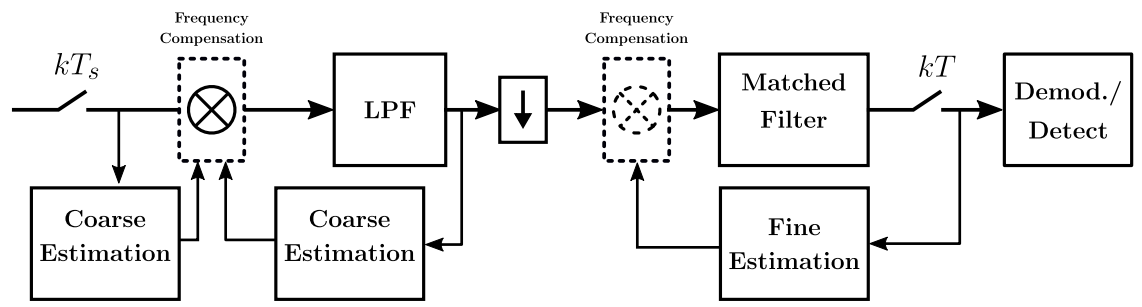

Figure 2.7: Coarse and fine carrier recovery (estimation and compensation) in the receiver

by the fine estimation and frequency compensation blocks in the right side of Figure 2.7.

At each step, the range of the offset that can be estimated depends on the sampling frequency which is also proportional to the filter before that step. The wider the filter is (the higher sampling frequency), the larger is the range of the frequency offset that can be compensated. A wider filter means a larger noise bandwidth; therefore, although the range of tolerable offset increases the estimation error increases [68]. The carrier recovery may even need to change the control voltage of the Voltage Controlled Oscillator (VCO) to change the Local Oscillator (LO) frequency in the analog domain. To eliminate the complexity and power consumption of such techniques, offset tolerant demodulators have been proposed.

\subsubsection{Tolerating Frequency Offset}

Offset tolerant demodulators may change the decision making process to be insensitive to the frequency offset. They may also only try to alleviate the effect of frequency offset by some compensation methods on the decision variables inside the demodulator. As mentioned in Chapter 1, in this work we focus on scalable offset tolerant demodulators. In an absolute sense, scalability can be defined as tolerating arbitrary large frequency offsets without any loss of BER performance. In other words, the demodulator should lead to the same BER value for a specific $E_{b} / N_{0}$, independent of the frequency offset. A simple diagram which shows the concept of an offset tolerant demodulator is shown in Figure 2.8. The complex notations are used here for simplicity.

In Figure 2.8, a frequency offset equal to $\omega_{O}$ is assumed. It can be seen that due to the frequency offset, the input to the baseband processing has a frequency of $\omega_{O}$ which in an ideal situation would be zero. The Low-Pass Filter (LPF) is designed so that it captures the signal in the presence of the frequency offset. After the filter, the signal is sampled and sent to the demodulator without applying frequency estimation and compensation blocks.

The sampling frequency $F_{s}=1 / T_{s}$ ( $T_{s}$ is the sampling period) which is twice (or 


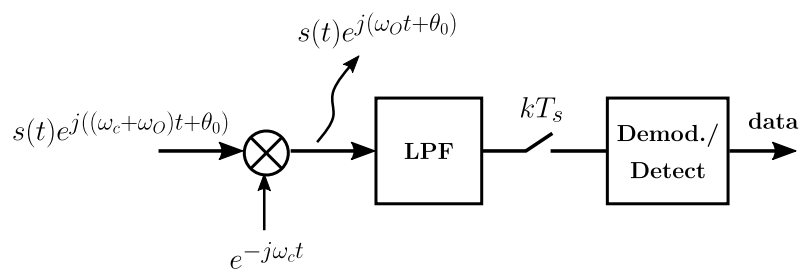

Figure 2.8: Simple block diagram of a receiver with offset tolerant demodulator

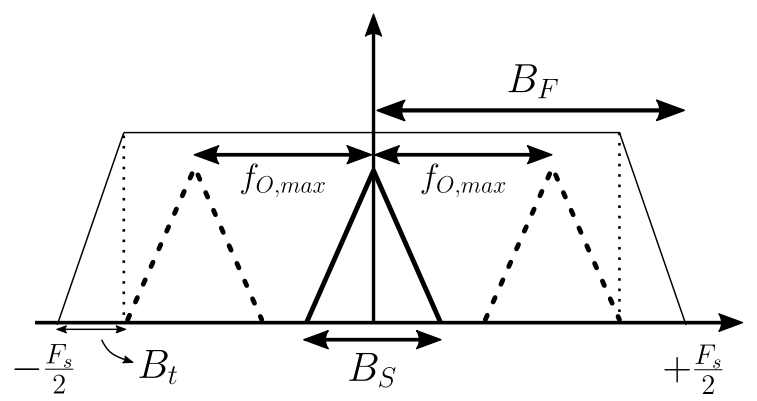

higher than) the filter bandwidth to avoid noise folding and aliasing. The relation between the filter bandwidth, minimum sampling frequency and the tolerable frequency offset are shown in Figure 2.9. In this figure, $B_{F}$ is the filter bandwidth, $F_{s}$ is the sampling frequency with complex samples and the signal bandwidth is denoted by $B_{S}$. If the demodulator has scalable offset tolerance, the only factor which determines the frequency offset will be the filter bandwidth. Focusing on this interpretation of a scalable frequency offset tolerance, the next chapter investigates offset tolerant demodulators for ultra-narrowband communications and their limitations for scalable offset tolerance. 


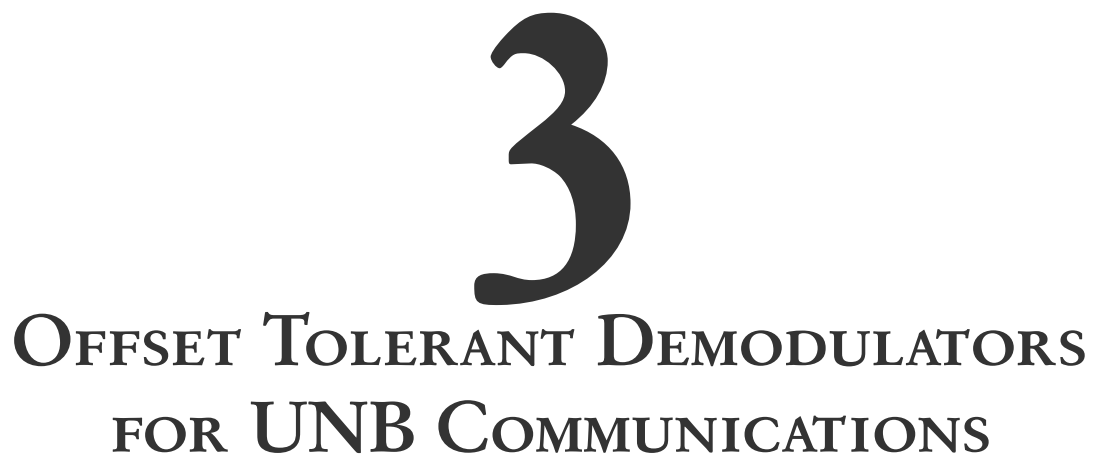

\begin{abstract}
AвsTRACT - To achieve scalable offset tolerance, this chapter focuses on demodulators for FSK and PSK which have the potential of tolerating large frequency offset. Two offset tolerant demodulators are investigated for FSK and PSK in the presence of frequency offset which can be several times the symbol rate. For FSK modulation, an existing DFT-based demodulator is modified considering UNB applications. For PSK, a Double Differential scheme (DDPSK) is introduced which is a known offset tolerant demodulator. To ensure a fair comparison between these two demodulators, a timing synchronization for DDPSK is designed and used in our simulations. These demodulators are simulated for large frequency offset and their BER performance and complexity are compared. Particularly, the effect of a wide filter required to capture the signal in the presence of a large frequency offset is considered. The trade-off between BER performance and complexity for different filter bandwidths is discussed. Both methods work independent of frequency offset, however, it is shown that a wide filter degrades the BER performance of DDBPSK, whereas, the DFT-based demodulator for FSK is not affected by filter bandwidth. This robustness is obtained at the expense of increased computational load. The results clarify the limitations of these two demodulators which will be dealt with in the next chapters.
\end{abstract}

\title{
3.1 INTRODUCTION
}

Offset tolerant demodulators have been proposed to tackle the frequency offset problem for both FSK and PSK modulations. Low data rate applications where a signal experiences a large Doppler shift, such as satellite communications, are applications of offset tolerant demodulators [39, 44, 106]. Moreover, in some

This chapter is based on [S:3]. 
other applications like Bluetooth, the receivers are expected to be able to afford frequency uncertainty. This enables designers to achieve a low cost and low power receivers by simplifying frequency generation [47]. In this chapter, the offset tolerant demodulators are considered for ultra-narrowband communications. As mentioned earlier, our target is to achieve scalable offset tolerance. For this purpose, two offset tolerant demodulators for FSK and PSK, which can potentially tolerate a relatively large frequency offset (multiple times the symbol rate), are investigated. These two demodulators are simulated for different ranges of tolerable frequency offset and their BER performance and complexity are evaluated and compared. This helps us to find out the limitations of these demodulators and address them in the next chapters.

For FSK modulated signals, different offset tolerant demodulators have been proposed. A review on these demodulators (presented in the next section) shows that a demodulator based on the Discrete Fourier Transform (DFT) [44] has the potential of achieving scalable offset tolerance. The target of the demodulator in [44] is to tolerate largely varying Doppler shift which is faced in Low Earth Orbit (LEO) satellite communications. Since UNB communication is mostly used for fixed nodes, a largely varying Doppler shift is not a concern. Therefore, we introduce a modified version of the DFT-based demodulator here. To enable window synchronization when a frequency offset is experienced, a DFT-based window synchronization algorithm is proposed in [44]. For tolerating large frequency offset using the DFT-based demodulator, it must be implemented together with this window synchronization, thus, this algorithm is discussed here as a phase of the demodulator operation.

For offset tolerant PSK demodulation, the concept of Double Differential encoding and an autocorrelation based demodulator (ACD) has been introduced in [94]. It is an extension to the concept of Differential PSK (DPSK) which removes the phase offset. DDPSK can remove the effect of frequency offset regardless of its value; therefore, it is used as an offset tolerant PSK demodulator in the analyses of this chapter. As mentioned above, the DFT-based demodulator uses a window synchronization algorithm. To ensure a fair complexity comparison for FSK and PSK offset tolerant demodulators, the synchronization of the samples of a symbol in DDPSK must be considered as well. For this purpose, a simple synchronization method for DDPSK is introduced and used in this chapter.

In the next step, the modified DFT-based demodulator for FSK and the DDPSK with the proposed synchronization method are evaluated for different ranges of frequency offset tolerance and their BER performance and complexity are investigated. This analysis clarifies the limitations of these two demodulators and pinpoints the challenges that need to be addressed for a scalable offset tolerance.

This chapter is organized as follows. In the next section, a short overview of offset tolerant demodulators for FSK is presented followed by an explanation of the DFT-based demodulator for FSK and its modified version in section 3.3. In section 3.4, the DDPSK demodulator and our proposed synchronization scheme 
for that are presented. The BER performance and complexity of the DFT-based and the DDPSK demodulators as well as a discussion on their limitations in the presence of a large frequency offset are included in section 3.5. Finally, the concluding remarks can be found in section 3.6.

\subsection{Related Work}

As explained in Chapter 2 typical demodulators for FSK (including those explained in Chapter 2) are sensitive to frequency offset. For instance, a frequency offset equal to $6 \%$ of the data rate in a zero-crossing demodulator leads to $4.8 \mathrm{~dB}$ degradation in the BER performance [61]. To improve the performance, offset tolerant demodulators have been introduced $[19,43,50,56,75,102]$.

As explained in Chapter 2, a group of FSK demodulators converts the input signal into a square wave and generates a short length pulse on each transition. Then, the pulses are averaged with a low-pass filter. The voltage at the output of the filter is proportional to the density of the pulses which is an indication of the signal frequency. Thus, a decision on the transmitted frequency can be made based on the output of the filter $[43,102]$. In this type of demodulators, a frequency offset is converted to a DC offset in the output of the demodulator. Therefore, the problem of frequency offset cancellation is changed to DC-offset cancellation. In [102], a modified version of a zero-crossing demodulator is introduced with DC-offset cancellation which is able to tolerate a frequency offset up to $60 \%$ of the frequency separation when the IF frequency is $2 \mathrm{MHz}$, the data rate is $1 \mathrm{Mbps}$ and the frequency separation is at least $320 \mathrm{kHz}$. In [43], a phase quantization based FSK demodulator is designed which uses a DC-offset cancellation method to achieve an offset tolerance of $\pm 200 \mathrm{kHz}$ with a data rate more than $1 \mathrm{Mbps}$ and a modulation index of $h=0.25$.

For a DLL-based demodulator (explained in section 2.2, frequency offset causes variation in the period of the IF signal such that it does not match the delay on which the DLL is locked. To tolerate frequency offset, the delay line can be tuned by adding extra delay cells [19]. However, it adds analog complexity which increases power consumption. For example, to achieve an offset tolerance equal to $3 \%$ of the IF frequency ( $3 \mathrm{MHz}$ IF frequency when the data rate is $1 \mathrm{Mbps}$ and the frequency separation is $160 \mathrm{kHz}), 16$ delay stages are required for correction in [19]. In a different design [50], to achieve $\pm 350 \mathrm{kHz}$ offset tolerance with a $5 \mathrm{MHz}$ IF signal (the data rate is $250 \mathrm{kbps}$ or $1 \mathrm{Mbps}$ ), 64 delay stages are used. Thus, the scalability of such methods is questionable as it requires additional analog circuitry which is not desired.

In most of these methods, the maximum tolerable frequency offset is less than the frequency separation or the symbol rate. For those methods where frequency offset is converted to a DC-offset, tolerating a large frequency offset (even if possible in the design) requires a large dynamic range for the output of the averaging filter [56]. The achieved dynamic range is limited due to analog design consid- 
erations and a relatively low supply voltage (0.9-1.2 V supply). On the other hand, most of these methods depend on detecting the deviation of frequency from the IF frequency to detect the transmitted symbol. Therefore, the ratio of the frequency separation to the IF frequency can affect their performance. In the mentioned methods, the ratio of frequency separation to the IF frequency is between 0.016 to 0.4 . In a low data rate application assuming a data rate of $100 \mathrm{bps}$, with a frequency separation equal to the data rate $(100 \mathrm{~Hz})$ and an IF frequency of $1 \mathrm{MHz}$, this ratio will be 0.0001 which makes demodulator design more challenging. Scaling the frequency offset tolerance to large values (multiple time the data rate) in these designs (if possible at all) requires additional analog circuits.

In [44], a digital FSK demodulator is proposed based on the short-time DFT for LEO satellite communications. The Discrete Fourier Transform (DFT) is a well-known method to extract frequency components of a signal. When signal frequency components are time-variable, a concept called short-time DFT can be used. Short-time DFT is a time-frequency representation of a signal which shows the variation of signal components during time. The demodulator proposed in [44] uses a differential encoding in the transmitter and the symbols at the receiver are determined based on comparing the frequency components of two consecutive symbols. A variation of the DFT-based FSK demodulator is introduced in [39]. The receiver design is based on sub-sampling and one-bit quantization to decrease power consumption at the cost of BER performance degradation.

An FSK demodulator based on the DFT can potentially tolerate a large frequency offset if the sampling frequency and the size of the DFT increase $[44,61]$. Hence, it is a suitable option for a scalable offset tolerance. The DFT-based method introduced in [44] (also referred to as Hara et al here) uses differential encoding and a sub-optimum detection which makes it impossible to achieve the lowerbound of non-coherent FSK. The differential encoding and the complicated detection algorithm in [44] are utilized to tolerate large variations in frequency offset which is required by LEO satellite communication. The largely varying frequency offset is not the case for the target application in LPWANs as the nodes are mainly fixed. In the next section, a DFT-based demodulator is introduced which fits UNB communication and achieves a BER performance equal to the non-coherent FSK demodulator.

\subsection{The DFT-based Demodulator}

The general concept of the proposed method is similar to [44] with a variation in the detection algorithm. In this section, we start with explaining the general concept of the DFT-based demodulator and the next two subsections explain the detection and synchronization algorithm, respectively.

The block diagram of the DFT-based demodulator introduced in [44] is shown in 


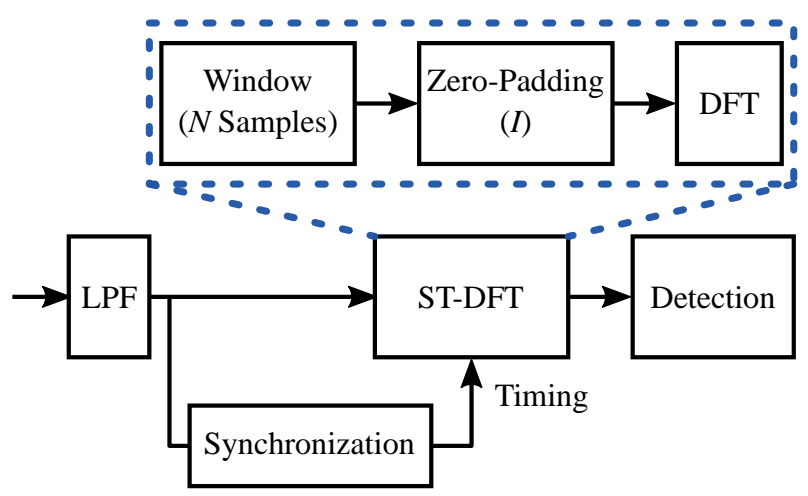

Figure 3.1: The block diagram of the demodulator proposed in [44]

Figure 3.1. The received samples pass through a low-pass filter (LPF) before the demodulator. Then, the samples are sent to a Short-Time Discrete Fourier Transform (ST-DFT) analysis block which is receiving timing information from the synchronization block. Then, the detection algorithm provides a data sequence based on the output of the ST-DFT block.

The ST-DFT is achieved by calculating the DFT for a set of samples of a timevarying signal during different time instants. Here, ST-DFT analysis is simply calculating the DFT for the samples of one symbol to determine the frequency components in each symbol. To tolerate large frequency offset, the lowpass filter might be much wider than the signal bandwidth. Moreover, complying with the Nyquist criterion (with respect to the filter bandwidth) necessitates a higher sampling frequency. Considering a sampling frequency $N$ times the symbol rate (which means $N$ samples per symbol), the input of the demodulator for the $m^{t h}$ symbol can be written as follows.

$$
r_{m}[n]=\sqrt{\frac{E_{S}}{N}} \exp \left(j\left(2 \pi\left(f_{m}+f_{O}\right) n T_{s}+\theta\right)\right),
$$

where $E_{S}$ is the energy per symbol, $r_{m}[n]$ is the $n^{\text {th }}$ sample $(n=0, \ldots, N-1)$ of the $m^{\text {th }}$ symbol. $f_{m}$ is the transmitted frequency during the $m^{t h}$ symbol which is either $f^{0}$ or $f^{1}$; $\theta$ is the phase offset, $f_{O}$ is the frequency offset and $T_{s}$ is the sampling period. The transmitted frequency $f_{m}=a_{m} f_{\text {sep }} / 2$ where $a_{m}= \pm 1$ is the transmitted bit (in bipolar format) for the $m^{t h}$ symbol and $f_{\text {sep }}$ is the frequency separation for BFSK. For proper operation of the demodulator, the set of samples belonging to one symbol should be selected. This requires a window synchronization which is explained later in subsection 3.3.2. For now, it is assumed that the $N$ samples of a symbol are selected correctly.

After the filter, the samples of a symbol are padded with zeros and sent to the 
DFT calculation block to achieve the following.

$$
X_{k, m}=\sum_{n=0}^{N I-1} \mathbf{x}_{m}^{I}[n] \exp \left(-j \frac{2 \pi k n}{N I}\right)
$$

where, $X_{k, m}$ is the $k^{t h}$ bin of the DFT calculated for the $m^{t h}$ symbol $(k=$ $0, \ldots, N I-1) . I$ is the zero-padding factor which means that $N$ samples of the signal are padded by $N(I-1)$ zeros to achieve a zero-padded sequence $\mathbf{x}_{m}^{I}$ with length $N I$ as the input of the DFT. Finally, the decision on the value of each transmitted symbol of BFSK is made based on the magnitudes of $X_{k, m}$.

Zero-padding is used to improve the bin resolution of the DFT and the BER performance. Notice that without zero-padding the bin resolution of the DFT, $F_{\text {res }}$, is equal to the symbol rate $R_{S}\left(F_{\text {res }}=F_{s} / N=R_{S}\right)$. The frequency separation of the BFSK modulation is assumed to be equal to the symbol rate $\left(f^{1}=f_{c}+1 / 2 T\right.$ and $\left.f^{0}=f_{c}-1 / 2 T\right)$. This is the value that ensures the best BER performance while keeping the minimum bandwidth [44].

The magnitude of $X_{k, m}$ determines the contribution of the $k^{t h}$ DFT bin and its corresponding frequency to the signal during the $m^{\text {th }}$ symbol interval. In case of noise free operation, the maximum value of the DFT can be used to decide the frequency of the transmitted signal; however, for a noisy signal, the DFT bin corresponding to the transmitted frequency does not necessarily have the maximum value (w. r. t. the other bins) due to noise. It is particularly severe in this system as the DFT is calculated over a small set of signal samples in one symbol. Hence, a detection algorithm other than just a maximum search is required to extract correct data.

\subsubsection{Detection Algorithm}

To achieve a BER performance similar to the non-coherent FSK, two reasons that cause performance loss in [44] are eliminated. These include differential detection, which imposes a limit on the system performance, and relying on peak detection for decision making which together provide robustness against constant and largely varying frequency offset. Since tolerating a largely varying offset is not the target for (mainly) fixed nodes of the UNB systems, a simpler detection scheme can be used which is able to achieve the performance of a noncoherent FSK. In order to detect data in our detection scheme, the DFT bins corresponding to frequency for symbol 1 and $O\left(f^{1}\right.$ and $\left.f^{0}\right)$ of the employed BFSK modulation are needed.

The bins corresponding to $f^{1}$ and $f^{0}\left(k_{1}\right.$ and $k_{0}$, respectively) are calculated once in a packet during the window synchronization phase which uses the same window synchronization algorithm as the one used in [44] and is explained in the next section. Once $k_{1}$ and $k_{0}$ are known, the detection algorithm for each symbol is very simple. The decision is made by comparing the values of the two 


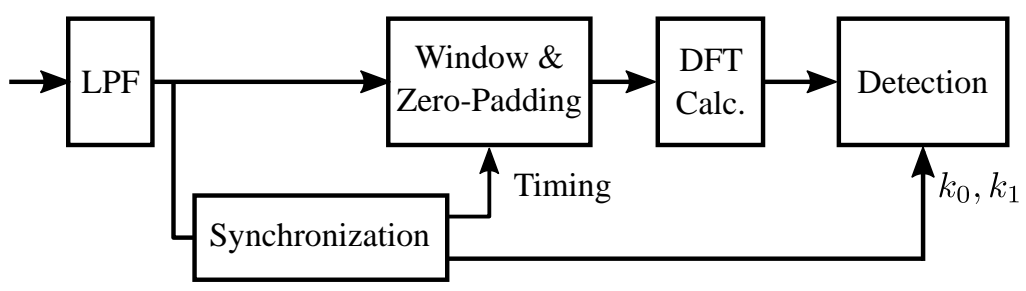

Figure 3.2: Block diagram of the DFT-based demodulator

DFT bins.

$$
\hat{a}_{m}= \begin{cases}+1 & X_{k_{1}, m}>X_{k_{0}, m} \\ -1 & X_{k_{1}, m}<X_{k_{0}, m}\end{cases}
$$

Figure 3.2 depicts the modified DFT-based demodulator. The window synchronization block determines the window timing, $k_{0}$ and $k_{1}$. The detection block uses the DFT values and (3.3) to detect the received symbols.

\subsubsection{WindOW SYNCHRONIZATION}

As mentioned earlier, a DFT-based window synchronization algorithm has been proposed in [44] which is necessary for proper functioning of the demodulator in the presence of a large frequency offset. In this chapter, we also use the same window synchronization algorithm. Due to its effect on the complexity of the DFT-based demodulator, this algorithm is explained in detail in this section. This explanation is also helpful to understand the method proposed in Chapter 5.

For synchronization, a preamble of alternating ones and zeros (1010...) is used. The number of symbols in the preamble is denoted by $L$. For each symbol, windows with different delay values, between 0 and $N-1$, are considered. Only one of these windows is fully aligned and others include samples of two consecutive symbols. Each window includes a sequence of samples as follows.

$$
\mathbf{x}_{m, i}=\left[r_{m}[i], \ldots, r_{m}[N-1], r_{m+1}[0], \ldots, r_{m+1}[i-1]\right]
$$

The $r_{m}[i]$ is the same as in (3.1) while $\mathbf{x}_{m, i}$ shows the set of samples for symbol $m$ and window delay of $i$. The DFT is calculated for each set of samples as follows.

$$
X_{k, m}^{i}=\sum_{n=0}^{N I-1} \mathbf{x}_{m, i}^{I}[n] \exp \left(-j \frac{2 \pi k n}{N I}\right)
$$

where $X_{k, m}^{i}$ is the DFT value for the $k^{t h}$ bin and the $m^{\text {th }}$ symbol when a window with delay $i$ is considered. $\mathbf{x}_{m, i}^{I}$ is the zero-padded sequence achieved from $\mathbf{x}_{m, i}$. 


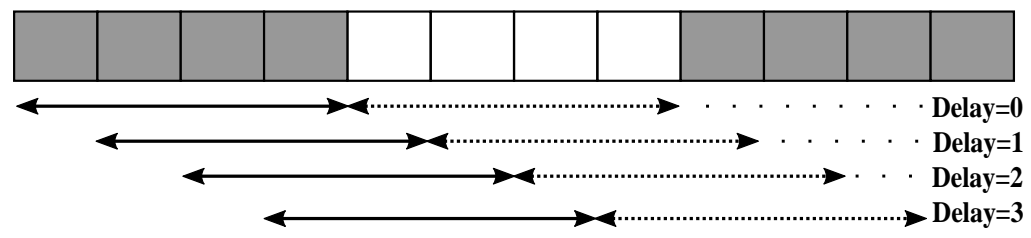

Odd Symbols ( $\square$ ) $\square$ Even Symbols ( ...... )
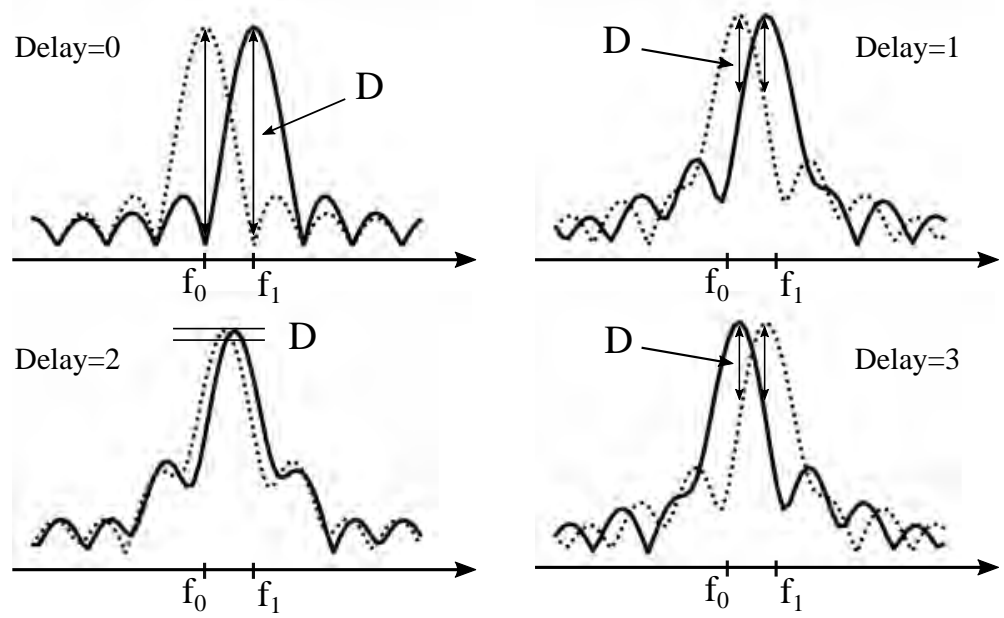

Figure 3.3: Three symbols of a preamble, the windows of different delay values for the first two symbols and an example of spectrum variation when there are 4 samples per symbol. Solid and dotted lines show windows and spectra of odd and even symbols, respectively.

Figure 3.3 illustrates three symbols of the preamble and the windows in case of four samples per symbol (each square is a sample). It also shows how the spectrum varies for different delays. The double-headed arrows in the top part of the figure denote windows. Each row of arrows corresponds to a certain delay value as shown in the figure. Solid (dotted) arrows show the windows for an Odd (Even) symbol with different delay values. The symbols within the preamble are called Even and Odd depending on their index $m=1, \ldots L$.

When $X_{k, m}^{i}$ is calculated for $m=1, . ., L, k=0, \ldots, N I-1$ and $i=0, \ldots, N-1$, for each delay $i$, all DFT magnitudes corresponding to Odd symbols (Even symbols) in the preamble are added together to achieve the following.

$$
S_{i}^{O}(k)=\sum_{n=1}^{L / 2}\left|X_{k, 2 n-1}^{i}\right|
$$




$$
S_{i}^{E}(k)=\sum_{n=1}^{L / 2}\left|X_{k, 2 n}^{i}\right|
$$

In fact, (3.6) and (3.7) calculate the sum of the DFT magnitudes for solid windows in Figure 3.3 and the sum of those for dotted windows with the same delay (or in each line of arrows as shown in Figure 3.3), respectively.

To synchronize the window, the delay value for which $R_{i}$ in the following equation is maximized needs to be found $[44,89]$.

$$
R_{i}=\left[S_{i}^{E}\left(k_{i}^{E}\right)-S_{i}^{E}\left(k_{i}^{O}\right)\right]+\left[S_{i}^{O}\left(k_{i}^{O}\right)-S_{i}^{O}\left(k_{i}^{E}\right)\right],
$$

where $k_{i}^{E}$ and $k_{i}^{O}$ are the bins with maximum magnitude in $S_{i}^{E}$ and $S_{i}^{O}$, respectively. Finding the maximum of (3.9) is simply finding the delay value which maximizes the difference shown by $D$ in Figure 3.3. In addition to the timing information (window delay), the bins corresponding to two symbols are achieved as follows and sent to the detection block.

$$
k_{1}=k_{i}^{O} \text { and } k_{0}=k_{i}^{E}
$$

\subsubsection{BeR Performance and the Zero-padding Factor}

Zero-padding is used to increase the bin resolution of the DFT and affects the BER performance of the DFT-based demodulator. Since the DFT is calculated using samples over a symbol, the bin resolution of the DFT without zero-padding equals to the symbol rate $\left(F_{s} / N=R_{S}\right)$. Since the frequency separation of the FSK modulation is $R_{S}$, the DFT bins corresponding to the two BFSK frequencies are two adjacent bins in case of no zero-padding. Due to frequency offset, the frequencies of the BFSK symbols may not match the DFT bins which leads to spectral leakage. In such cases, a low bin resolution causes erroneous detection in presence of noise. The worst case is when the bins corresponding to the FSK symbol frequencies are exactly in the middle of two DFT bins. The BER in this situation depends on the bin resolution and can be improved by increasing the zero-padding factor.

To show the effect of the zero-padding factor on the BER performance, the demodulator in Figure 3.2 is simulated for different zero-padding factors and the worst case offset for that zero-padding. In these simulations, the preamble length $(L)$ and the number of samples per symbol $(N)$ are 16 and 8, respectively, similar to [44] (the BER performance for other values of $N$ are discussed in the next section). The BER curves for different zero-padding factors are shown in Figure 3.4. The BER curve for a non-coherent BFSK, which is the lower bound, is also demonstrated in Figure 3.4. According to Figure 3.4, at BER $=0.1 \%, I=8$ requires less than $5 \%$ more power compared to the non-coherent FSK. Thus, we use $I=8$ in the rest of this chapter. 


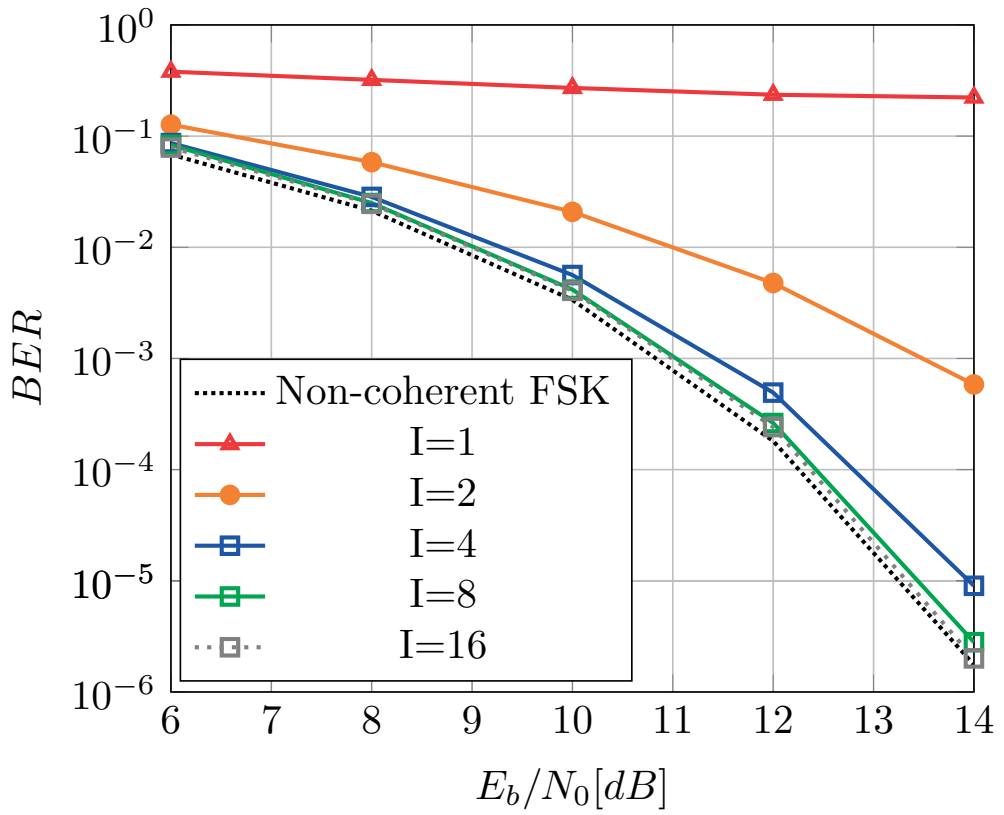

Figure 3.4: The BER performance of the DFT-based demodulator for different zero-padding factors

\subsection{Double Differential PSK}

\subsubsection{The Autocorrelation Based Demodulator}

To eliminate the effect of the frequency offset on PSK detection, Double Differential signaling has been proposed [94] resulting in Double Differential PSK (DDPSK). The idea is an extension of the same idea for Differential PSK (DPSK). In DPSK, the phase of each symbol is compared with the phase of the previous symbol to achieve robustness against constant phase offset. Now, to remove the effect of the frequency offset (a variable phase offset), we can add another differential encoder/decoder to the DPSK modulator/demodulator. An offset tolerant demodulator for DDPSK has been introduced based on the correlation of a signal with its delayed version. The is called Auto-Correlation Demodulator (ACD). The block diagram of an ACD for DDPSK is shown in Figure 3.5.

The baseband PSK signal after the Low-Pass Filter $(r(t)$ in Figure 3.5) and during the $n^{t h}$ symbol period is as follows.

$$
r(t)=\sqrt{\frac{E_{S}}{T}} e^{j\left(\omega_{O} t+\varphi_{n}+\theta_{0}\right)}+n(t),
$$



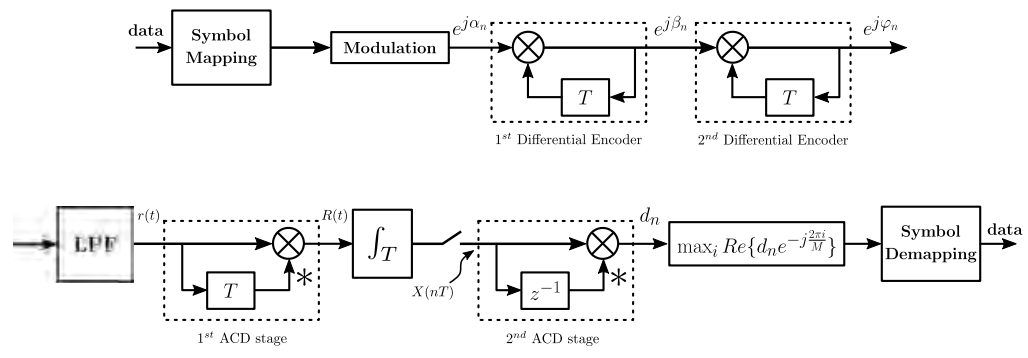

Figure 3.5: The block diagram of a Double Differential PSK (DDPSK) modulator and ACD for DDPSK

where $E_{S}$ is the energy per symbol, $T$ is the symbol period, $\omega_{O}$ is the frequency offset, the $\varphi_{n}$ is the modulated phase for the $n^{\text {th }}$ symbol, $\theta_{0}$ is a constant phase offset and $n(t)$ represents the complex noise process. In the above equation, a rectangular pulse shape is considered for the PSK modulator and it is assumed that the received signal is not distorted by the filter. This is a reasonable assumption when the filter bandwidth is large enough to include the signal bandwidth completely [94]. The received signal $r(t)$ is multiplied by the conjugate of the signal which is delayed by one symbol period to achieve $R(t)$ as follows.

$$
R(t)=r(t) r(t-T)^{*}=\frac{E_{S}}{T} e^{j\left(\omega_{0} T+\Delta \varphi_{n}\right)}+n^{\prime}(t),
$$

where $T$ is the symbol period and $r(t-T)$ is the signal during the $(n-1)^{t h}$ symbol. $\Delta \varphi_{n}=\varphi_{n}-\varphi_{n-1}$ is the difference between the modulated phase for the $n^{t h}$ and the $(n-1)^{t h}$ symbols. Integrating $R(t)$ over a symbol period, $X(n T)$, which is the output of the first stage of the ACD for the $n^{t h}$ and the $(n-1)^{t h}$ symbol can be expressed as follows.

$$
X(n T)=E_{S} e^{j\left(\omega_{0} T+\Delta \varphi_{n}\right)}+\tilde{n}^{\prime},
$$

where $\tilde{n}^{\prime}$ is the integral of the noise process and is a complex random variable. As can be seen, the frequency offset term is removed and is converted to a constant phase offset which depends on the frequency offset $\left(\omega_{O} T\right)$. This constant phase term can be eliminated with a stage of differential detection which forms the second stage of the ACD in Figure 3.5. The output of the second stage, which is the decision variable for the $n^{\text {th }}$ received symbol, is achieved as follows.

$$
d_{n}=X(n T) X^{*}((n-1) T)=E_{S}^{2} e^{j \Delta^{2} \varphi_{n}}+n^{\prime \prime}
$$

where $\Delta^{2} \varphi_{n}=\Delta \varphi_{n}-\Delta \varphi_{n-1}$ and $n^{\prime \prime}$ represents the noise components. As can be seen, the decision variable achieved in this way is only dependent on the double 
difference of $\varphi_{n}$. To be able to detect the transmitted information from the decision variable in (3.13), the information is encoded in the double difference of the phases of the transmitted symbols. A double differential encoder for MPSK is depicted in Figure 3.5. From Figure 3.5 it can be concluded that, the encoder is achieved from cascading two differential encoders. Writing $\varphi_{n}$ according to Figure 3.5, we have:

$$
\begin{aligned}
& \alpha_{n}=\beta_{n}-\beta_{n-1} \\
& \beta_{n}=\varphi_{n}-\varphi_{n-1}
\end{aligned}
$$

Using (6.1), it can be shown that the phase of $d_{n}$ (which is $\Delta^{2} \varphi_{n}$ ) is actually $\alpha_{n}$. If $d_{n}$ is expressed as in (3.13), the transmitted symbol can be obtained using the following decision criterion which is the same one used for conventional MPSK detection [45].

$$
\hat{\alpha_{n}}=\underset{\alpha_{i} \in A}{\arg \max } \operatorname{Re}\left[d_{n} \times \exp \left(-j \alpha_{i}\right)\right]
$$

where $A=\left\{\frac{2 \pi i}{M} \mid i=0, \ldots, M-1\right\}$ is the set of possible symbol phases for MPSK modulation. At least a $2 \mathrm{~dB}$ performance loss is the sacrifice compared to DPSK, to achieve frequency offset tolerance with DDPSK. A simple method to improve the performance of the DDPSK technique is proposed in [94]. The improvement is achieved by changing the delay of the second stage of the double differential encoder and the first stage of the ACD demodulator from $T$ to $2 T$. This extra delay changes the noise samples involved in the two stages of differential decoding and decreases their correlation [93]. As a result, the BER performance improvement is obtained without any additional complexity.

\subsubsection{Synchronization FOR PSK}

In the previous section, a window synchronization algorithm for the DFT-based demodulator for FSK was explained. The window synchronization algorithm is based on selecting the best set of samples. To have a fair comparison between the complexity of the two methods, a synchronization algorithm for PSK is introduced in this section and used in our simulations.

A discrete time model of a DDPSK demodulator in Figure 3.5 is shown in Figure 3.6. It is assumed that sampling frequency is $N$ times the symbol rate (there are $N$ samples per symbol). The sampling frequency is proportional to the bandwidth of the LPF prior to the demodulator and also determines the offset tolerance of the filter (this will be discussed in the next section). For each incoming sample at the input of the demodulator, a value is generated which is a sample of the output of the first stage. The summation (an integrate and dump filter) needs to 


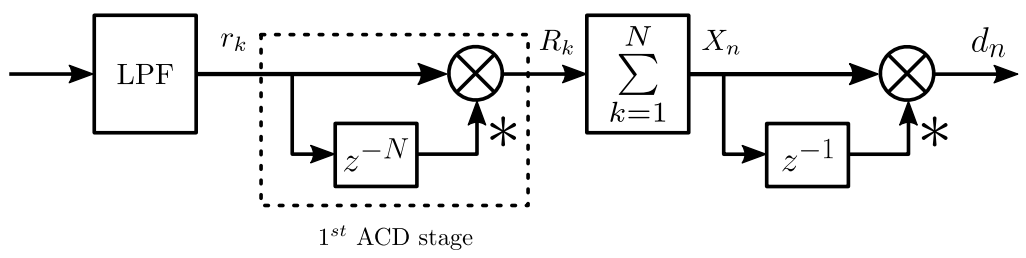

Figure 3.6: The block diagram of ACD for DDPSK

sum $N$ samples at the output of the first stage and sends the result to the next stage. To provide $X_{n}$ (which is derived from the $n^{t h}$ and the $(n-1)^{t h}$ symbols), the summation block should add the correct set of samples which all have the same information phase $\Delta \varphi_{n}$.

The challenge of selecting the correct set of samples for summation is similar to the window synchronization problem for the DFT-based demodulator. To clarify, the sequence of samples at the output of the first stage are shown in Figure 3.7 when $N=4$. The shown samples are derived from symbols $n-2$ to $n+1$ (e.g. $\Delta \varphi_{n-1}=\varphi_{n-1}-\varphi_{n-2}$ is from symbols $n-1$ and $n-2$ ). The possible selections for the sets of samples to be added in the summation block are shown by double headed arrows. These resemble the window positions discussed in the window synchronization algorithm for FSK demodulator in section 3.3.2 (see Figure 3.3). For simplicity these possible selections (double headed arrows in Figure 3.7) are called windows here.

Each line of the arrows demonstrates a specific delay value for the windows (a specific offset value for integrate and dump in terms of samples). As can be seen in Figure 3.7, only one of the set of windows are aligned to a set of samples such that all samples in the set have the same phase information $\left(\Delta \varphi_{n-1}, \Delta \varphi_{n}\right.$ or $\left.\Delta \varphi_{n+1}\right)$. These windows are shown by solid black double headed arrows. The rest of the windows include samples with different phase information. For instance, the middle dashed red arrow in the figure includes three samples with $\Delta \varphi_{n}$ and one sample with $\Delta \varphi_{n-1}$. Thus, using the windows shown by the dashed red arrows leads to inter-symbol interference and degrades the performance of the demodulator.

In the following, we derive a synchronization for PSK which targets at selecting a set of samples (correct window) which all have the same phase information $(\Delta \varphi)$. According to (3.10), the input samples of the demodulator during the $n^{t h}$ symbol are as follows.

$$
r_{k}=\sqrt{\frac{E_{S}}{N}} e^{j\left(\omega_{0} k T_{s}+\varphi_{n}+\theta_{0}\right)}+\eta_{k},(n-1) N<k \leq n N
$$

where $r_{k}$ is the $k^{\text {th }}$ sample of the input of the demodulator, $\varphi_{n}$ is the modulated phase during the $n^{t h}$ symbol and $T_{s}$ is the sampling period. $\eta_{k}$ are noise samples. 


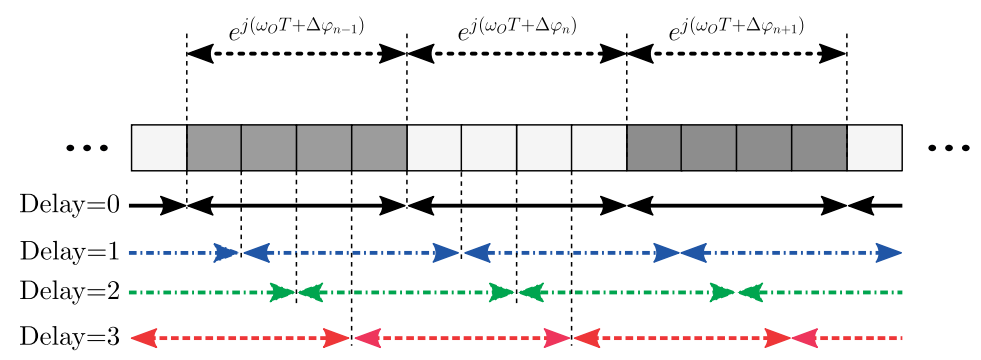

Figure 3.7: The sequence of samples at the output of the first stage. Double headed arrows show the possible selections for the sets of samples to be added in the summation block.

Using (3.11) and (3.16), the samples at the output of the first stage when the $n^{\text {th }}$ and the $(n-1)^{t h}$ symbols are multiplied are as follows.

$$
R_{k}=r_{k} r_{k-N}^{*}=\frac{E_{S}}{N} e^{j\left(\omega_{O} T+\Delta \varphi_{n}\right)}+\eta_{k}^{\prime},
$$

where $\eta_{k}^{\prime}$ represents the noise components. Every $N$ samples, the phase of $R_{k}$ changes depending on the difference between the modulated phase of the received symbols, $\Delta \varphi_{n}$. A sequence of $3 N$ samples of $R$ is shown in Figure 3.8. Double headed arrows show windows for three different delays, $0, d$ and $N-1$. The sums over the two first windows with delay $d$, which are shown by $w_{1}(d)$ and $w_{2}(d)$ are calculated as follows (assuming no noise).

$$
\begin{aligned}
w_{1}(d) & =\sum_{i=d+1}^{N+d} R_{i} \\
& =\sum_{i=d+1}^{N} \frac{E_{S}}{N} e^{j\left(\omega_{O} T+\Delta \varphi_{n-1}\right)}+\sum_{i=N+1}^{N+d} \frac{E_{S}}{N} e^{j\left(\omega_{O} T+\Delta \varphi_{n}\right)} \\
& =(N-d) \frac{E_{S}}{N} e^{j\left(\omega_{O} T+\Delta \varphi_{n-1}\right)}+d \frac{E_{S}}{N} e^{j\left(\omega_{O} T+\Delta \varphi_{n}\right)} \\
w_{2}(d) & =\sum_{i=N+d+1}^{2 N+d} R_{i} \\
& =\sum_{i=N+d+1}^{2 N} \frac{E_{S}}{N} e^{j\left(\omega_{0} T+\Delta \varphi_{n}\right)}+\sum_{i=2 N+1}^{2 N+d} \frac{E_{S}}{N} e^{j\left(\omega_{O} T+\Delta \varphi_{n+1}\right)} \\
& =(N-d) \frac{E_{S}}{N} e^{j\left(\omega_{0} T+\Delta \varphi_{n}\right)}+d \frac{E_{S}}{N} e^{j\left(\omega_{0} T+\Delta \varphi_{n+1}\right)}
\end{aligned}
$$




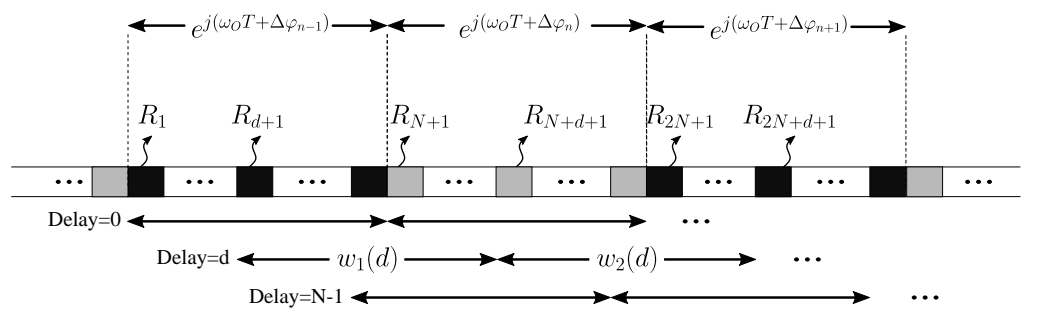

Figure 3.8: A sequence of $3 N$ samples of $R$. Double headed arrows show windows for three different delays, $0, d$ and $N-1$.

Now, assume that the preamble is designed such that the $\Delta \varphi_{n}=\varphi_{n}-\varphi_{n-1}$ toggles between 0 and $\pi$ for each $N$ samples. For instance, in Figure 3.8, let's consider $\Delta \varphi_{n-1}=0, \Delta \varphi_{n}=\pi$ and $\Delta \varphi_{n+1}=0$. So the black and grey squares show the samples of $R$ for which $\Delta \varphi=0$ and $\Delta \varphi=\pi$, respectively. Replacing the values for $\Delta \varphi_{n-1}, \Delta \varphi_{n}$ and $\Delta \varphi_{n+1}$ in (3.18) and (3.19) and noticing that $e^{j 0}=1$ and $e^{j \pi}=-1$, the following expressions are obtained.

$$
\begin{gathered}
w_{1}(d)=(N-2 d) \frac{E_{S}}{N} e^{j \omega_{O} T} \\
w_{2}(d)=-(N-2 d) \frac{E_{S}}{N} e^{j \omega_{O} T}
\end{gathered}
$$

It can be seen that $|w 1|=|w 2|=(N-2 d) \frac{E_{S}}{N}$ and it will be maximum if $d=0$. In general, every $N$ samples, the value of $R_{k}$ toggles between $\frac{E_{S}}{N} e^{j \omega_{O} T}$ and $-\frac{E_{S}}{N} e^{j \omega_{O} T}$. The magnitude of summation for a set of $N$ samples of $R_{k}$ is maximum when all of them have the same sign (see arrows for Delay $=0$ in Figure 3.8). Figure 3.9 shows the value of the summations over different windows. The color of the circles corresponds to the color of the window which is represented by them (see Figure 3.7). The gray line shows how the magnitude of summation changes for different windows. As can be seen the magnitude has maximum value for black solid arrows in Figure 3.7.

Based on the above discussion, the synchronization algorithm can be summarized as follows. Assume that the length of the preamble is $L$, then the output of the first stage $R_{k}$ toggles $L-1$ times (every $N$ sample) during preamble reception. A moving average filter calculates the $w_{m}(d)$ as follows.

$$
w_{m}(d)=\sum_{i=(m-1) N+d+1}^{m N+d} R_{i}
$$

Then, for each delay value $d$ the $W(d)$ is calculated as follows. 


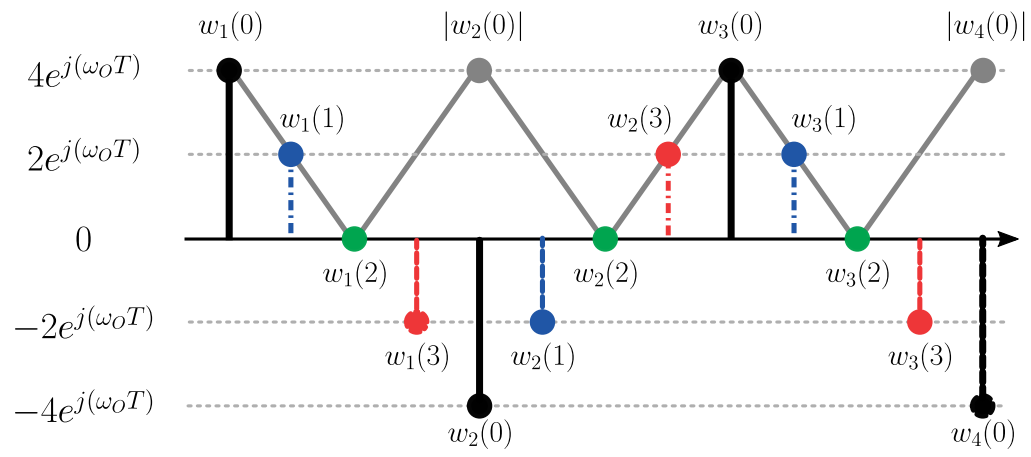

Figure 3.9: The values of summation over different windows of $R_{k}$ samples. The colors correspond to the window (arrow) colors in Figure 3.7.

$$
W(d)=\sum_{m=1}^{L-2}\left|w_{m}(d)\right|
$$

The delay value which maximizes the $W(d)$ is the correct delay and determines the proper set of samples for summation in Figure 3.6 to achieve $X_{n}$. Once this value is determined during the preamble, it can be used for the rest of the packet.

\subsection{Scaling Offeset Tolerance (BER and Complexity Evalu- ATION)}

\subsubsection{BER and Offret Tolerance}

Since the proposed method for FSK uses a window synchronization phase to acquire $k_{0}$ and $k_{1}$ (the bins corresponding to $f^{0}$ and $f^{1}$, respectively), the effect of frequency offset is already removed. As explained in the previous section, the Double Differential PSK (DDPSK) demodulator also eliminates the effect of frequency offset on the received signal. So in principle, both methods are capable of tolerating any value of frequency offset. As explained in Chapter 2, when an offset tolerant demodulator is used, the main parameter which determines the frequency offset tolerance is the bandwidth of the low-pass filter prior to the demodulator. A filter with bandwidth $B_{F}$ (similar to Chapter 2) is shown in Figure 3.10. The sampling frequency (for complex samples) is set to $F_{s}=2 B_{F}=F$ to avoid aliasing and noise folding. $B_{t}$ indicates the transition band of the filter which is non-zero for practical filters. The signal bandwidth and the maximum tolerable frequency offset are shown by $B_{S}$ and $\pm f_{O, \text { max }}$, respectively.

If $B_{t}=0$ (an ideal filter), the sampling frequency should meet the following criterion.

$$
F_{s} \geq B_{S}+2\left|f_{O, \max }\right|
$$




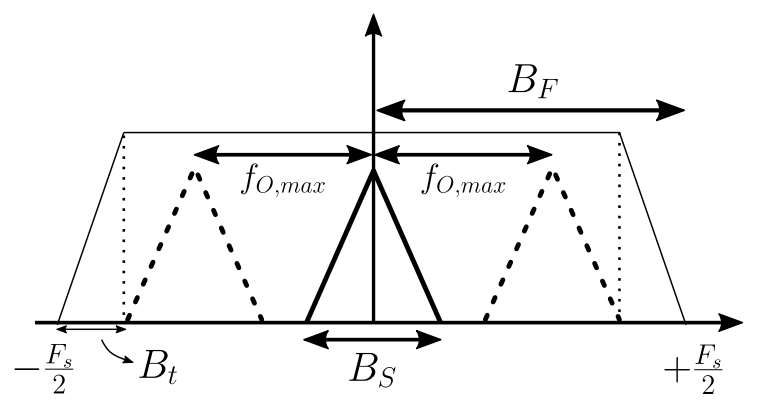

Figure 3.10: The relation between signal bandwidth $\left(B_{S}\right)$, sampling frequency $\left(F_{s}\right)$, filter bandwidth $\left(B_{F}\right)$ and maximum tolerable frequency offset $\left(f_{O, \max }\right)$

Here, we assume that the sampling frequency, $F_{s}=N R_{S}$, is an integer multiple of the symbol rate $R_{S}$ (equal to the bit rate for BFSK) and $N$ is the number of samples per symbol. Thus, according to (3.24), the amount of the frequency offset, which can be tolerated by the demodulator, is tuned only by changing the filter bandwidth, $B_{F}$ (and $N$ accordingly). The maximum tolerable frequency offset and the range of the normalized tolerable frequency offset can be obtained as follows.

$$
\begin{gathered}
\left|f_{O, \text { max }}\right|=R_{S} \frac{N-B_{S} / R_{S}}{2} \\
-\frac{N-B_{S} / R_{S}}{2} \leq \frac{f_{O}}{R_{S}} \leq+\frac{N-B_{S} / R_{S}}{2}
\end{gathered}
$$

The range of the tolerable frequency offset for each modulation scheme can be obtained by substituting the corresponding signal bandwidth in (3.26). The approximate null-to-null bandwidth $\left(B_{S}\right)$ of BFSK is $f_{\text {sep }}+2 R_{S}[103]$ and, here, the frequency separation is assumed to be equal to the symbol rate $\left(f_{\text {sep }}=R_{S}\right)$. Thus, the range of the tolerable frequency offset normalized to the symbol rate for BFSK is:

$$
-\frac{N-3}{2} \leq \frac{f_{O}}{R_{S}} \leq+\frac{N-3}{2}
$$

The offset tolerance of a DDPSK demodulator can be obtained in a similar way by replacing $B_{S}$ with the null-to-null bandwidth of PSK which is $2 R_{S}$ when rectangular pulse shaping is used [103]. It is worth mentioning that for a practical filter, $B_{t} \neq 0$ which decreases the offset tolerance for the same sampling frequency; nevertheless, using a larger $B_{F}$ to compensate for non-zero $B_{t}$ and consider $F_{s}=2 B_{F}$ extends offset tolerance to the desired value. 


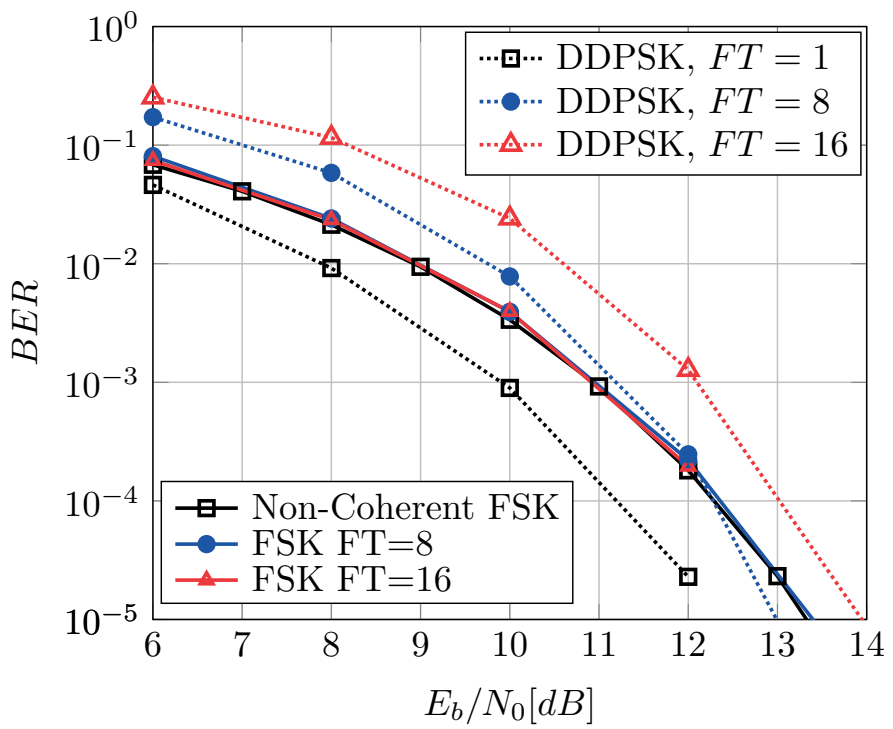

Figure 3.11: The BER curves for DFT-Based and DDPSK demodulator in presence of different filter bandwidths

In the following results, $F_{s}=F$ and its value normalized to the symbol rate, $F T$, is used for presenting the simulation results. The effect of increasing the filter bandwidth is modeled as increased noise bandwidth resulting in increased noise power levels. The distortion of the filter is neglected which is reasonable for large bandwidths. To show the performance of the offset tolerant demodulators for different filter bandwidths, the BER curves are derived for $F T=8,16$. The BER curves for the DFT-based FSK demodulator and the ACD for DDPSK are shown in Figure 3.11. The preamble length $L$ is set to 16 for both demodulators. For DDPSK the BER curve for $F T=1$ is also shown. Actually, $F T=1$ is also called coherent DDPSK when the LPF is exactly matched to the signal. However, this is not a realistic assumption in presence of a frequency offset and rectangular pulse shape [94]. We use $F T=1$ and no frequency offset as a reference of the best BER performance of the DDPSK demodulator. Because of the minimum filter bandwidth the noise power at the input of the demodulator is minimal as well [93]. Additionally, as a lower bound for the FSK demodulator performance, the BER curve of a non-coherent FSK demodulator [45] is also shown in Figure 3.11. It is the same as the non-coherent demodulator (correlator based demodulator) discussed in the previous chapter.

It can be seen that the BER curves for the DFT-Based demodulator match those of non-coherent BFSK detection. However, the BER performance of the DDPSK degrades when the filter bandwidth increases. For $F T=1$, DDPSK can achieve a BER performance which is $1 \mathrm{~dB}$ better than the DFT-Based demodulator. 


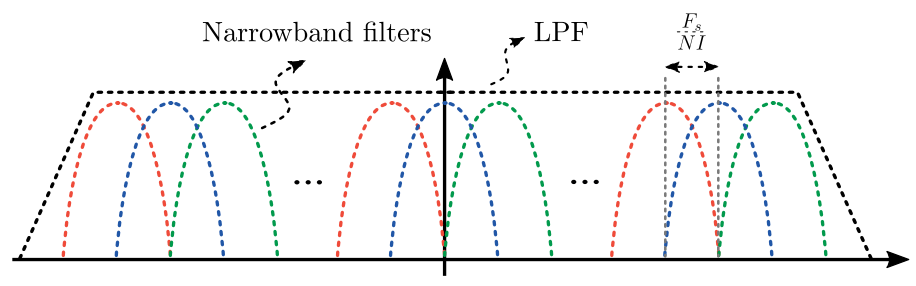

Figure 3.12: The filter bank interpretation of a DFT

Nonetheless, when $F T$ increases, the DDPSK performance degrades and becomes worse than the DFT-Based demodulator.

The robustness of the DFT-based demodulator against additional noise (included by a wider filter) can be justified via interpreting the DFT as a filter bank shown in Figure 3.12. The number of the narrowband filters equals the number of the DFT points which is proportional to the sampling frequency. Since the sampling frequency increases proportional to the filter bandwidth (so does the DFT points), the bandwidth of these narrowband filters remain constant regardless of the bandwidth of the LPF filter before the demodulator. Therefore, although the noise power increases at the output of the LPF, the noise power in each narrowband filter remains constant. The calculated DFT values and, thus, the performance of the demodulator is determined by the noise power in these narrowband filters [45]. As a result, increasing the bandwidth of the filter does not degrade the BER performance.

To have a better comparison for different values of $F T$, the $E_{b} / N_{0}$ required to achieve $B E R=0.1 \%$ is shown in Figure 3.13. According to Figure 3.13, the required $E_{b} / N_{0}$ does not change for the DFT-based demodulator while it increases for DDPSK. For $F T=16$ this difference is more than $1 \mathrm{~dB}$ and it increases for higher $F T$ values. For $F T=64$, the difference reaches to more than $3 \mathrm{~dB}$.

For a clear view on the effect of $F T$ on the offset tolerance, the BER of both demodulators is presented for different frequency offset values. Figure 3.14 shows the BER of the DFT-based demodulator and the DDPSK demodulator for $E_{b} / N_{0}=11 \mathrm{~dB}$ and $F T=8,16$. In these simulations the symbol rate is 100 Sym./s. The samples pass through a low-pass filter and, then, are decimated to have 8 and 16 samples per symbol depending on $F T=N$. The stop-band of the FIR low-pass filter used prior to the demodulator is set to $400 \mathrm{~Hz}$ and $800 \mathrm{~Hz}$ for $F T=8$ and $F T=16$, respectively $\left(B_{F}=400,800\right.$ as shown in Figure 3.10). Using (3.26), when $B_{t}=0$, the maximum tolerable frequency offset for PSK (FSK) is approximately calculated as $\pm 300 \mathrm{~Hz}( \pm 250 \mathrm{~Hz})$ and $\pm 700 \mathrm{~Hz}( \pm 650$ $\mathrm{Hz}$ ) for $N=8$ and $N=16$, respectively. As can be seen, the BER performance of the demodulators starts deteriorating from frequency offset values lower than the expected tolerance (around $200 \mathrm{~Hz}$ and $600 \mathrm{~Hz}$ ) which is a consequence of the non-zero $B_{t}$ of the filter. As expected, the BER does not change due to 


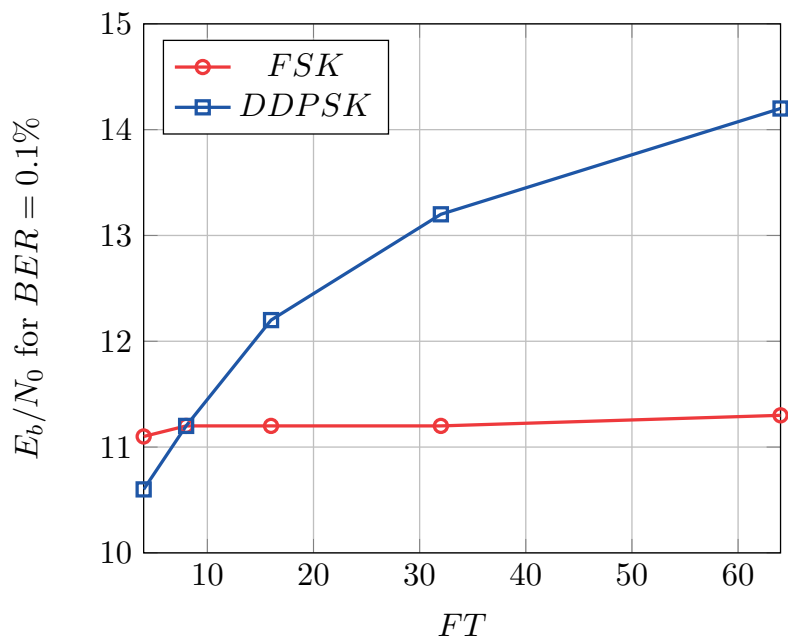

Figure 3.13: $E_{b} / N_{0}$ required for $B E R=0.1 \%$ for different $F T$

frequency offset as far as the signal is properly captured by the filter. The BER performance loss for DDPSK when $F T$ increases from 8 to 16 is also seen in this figure.

\subsubsection{COMPLEXITY}

In this section, an approximation of the complexity of the DDPSK and the FSK demodulators in terms of complex operations is derived. If $N$ is a power of two, $N I$ is also a power two (remember that $I=8$ is chosen here) and the DFT is calculated using a Fast Fourier Transform (FFT) which is an efficient method for implementing a DFT. In the following calculations, the zero-padding factor is shown as parameter $I$ for clarity. The preamble length is shown by $L$.

\section{DFT-based FSK demodulator}

For implementation of the DFT calculation block, we assume that the Fast Fourier Transform (FFT) algorithm is used. It is an efficient implementation of the DFT when the DFT points are a power of two. To calculate an NIpoint FFT which is required in the synchronization algorithm, $(N I / 2) \log _{2}(N I)$ complex multiplications and $N I \log _{2}(N I)$ complex additions are required. If $N$ (and thus, $N I$ ) is not a power of two, the $N I$ can be rounded to the next power of two for FFT calculations (it means adding more zeros). For instance if $N=24, N I=192$, the number of FFT points is considered to be 256 .

In the synchronization phase where the frequency bins for symbol detection are selected, for each preamble symbol, $N$ different FFTs are calculated which makes a total of $(L-1) N$ FFTs for synchronization. When the frequency bins 


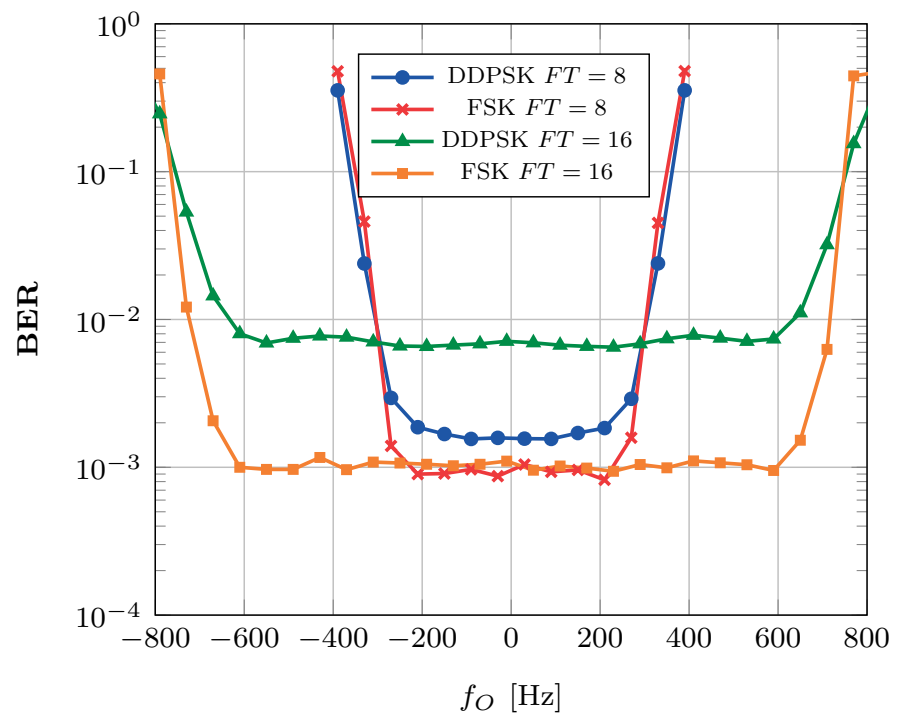

Figure 3.14: The BER versus frequency offset for the DFT-based and DDPSK demodulator when $F T=8,16$ and $E_{b} / N_{0}=11 \mathrm{~dB}$.

for detection are selected, in the detection phase only two bins of the DFT need to be calculated. In this case, calculating the complete FFT is not efficient. An implementation based on the basic DFT definition leads to $N$ complex multiplications and $N-1$ complex additions per DFT bin (notice that, due to zeropadding, only $N$ samples are non-zero when an $N I$-point DFT bin is calculated). Thus, overall $2 N$ complex multiplications and $2 N-2$ complex additions are needed to calculate the DFT bins for each symbol detection. It is worth mentioning that the conventional DFT-based demodulator as presented in [44] needs $(N I / 2) \log _{2}(N I)$ complex multiplications per symbol for detection as it needs all bins of the DFT.

\section{DDPSK}

For the synchronization of the DDPSK, during a preamble of length $L$, there are $(L-1)$ multiplications between symbols. Each symbol has $N$ samples so there are in total $N(L-1)$ complex multiplications $(N(L-1)$ different values for $\left.R_{k}\right)$. There are $N$ different delay values $(d=0, \ldots, N-1)$ for each window and according to (3.23), there are $L-2$ windows. As a result, in total $(L-2) N$ summations must be calculated. The sum of $R_{k}$ values for all possible window delays during the preamble can be implemented similar to a moving average filter. When $w_{q}$ for $\left\{R_{q}, R_{q+1}, \ldots, R_{q+N-1}\right\}$ is calculated, the sum over the next window $w_{q+1}\left(\left\{R_{q+1}, \ldots, R_{q+N-1}, R_{q+N}\right\}\right)$ is calculated using two complex additions as follows. 
Table 3.1: The number of Complex Multiplication (CM) and Complex Addition (CA) when $N$ is the number of samples per symbol, $I$ is the zero-padding factor for the FSK demodulator and $L$ is the preamble length.

\begin{tabular}{|c|c|c|c|}
\cline { 3 - 4 } \multicolumn{2}{c|}{} & Synchronization & Detection \\
\hline \multirow{2}{*}{ DFT-based demodulator } & $\mathrm{CM}$ & $\left((L-1) I N^{2}\right) / 2 \log _{2}(N I)$ & $2 N$ \\
\cline { 2 - 4 } & $\mathrm{CA}$ & $(L-1) I N^{2} \log _{2}(N I)$ & $2 N-2$ \\
\hline \multirow{2}{*}{ DDPSK } & $\mathrm{CM}$ & $N(L-1)$ & $N+1$ \\
\cline { 2 - 4 } & $\mathrm{CA}$ & $N(2 L-3)-3$ & $N-1$ \\
\hline
\end{tabular}

$$
w_{q+1}=w_{q}-R_{q}+R_{q+N}
$$

In this way, for the first window, $N-1$ complex additions are required and the rest of the $N(L-2)-1$ windows are calculated using two complex additions. Thus, in total, the synchronization for PSK needs $N(2 L-3)-3$ complex additions.

For detection of each symbol in DDPSK, the $N$ samples of two consecutive symbols are multiplied while in the second stage only one multiplication is needed. This leads to $N+1$ complex multiplications for detection of each symbol. Besides, $N-1$ complex additions are required to calculate the summation of $N$ values of $R_{k}$.

The complexity based on the number of Complex Multiplications (CM) and Complex Additions (CA) is presented in Table 3.1. The first column shows the operations needed for synchronization and the second column lists operations needed for detecting each symbol.

Complexity in terms of the number of Complex Multiplications (CM) for different values of $F T=N$ is shown for both methods in Figure 3.15 (notice that the vertical axis is on the logarithmic scale). Since the synchronization is done once in a packet, its complexity is divided by the packet length to achieve the complexity per symbol. To see the effect of different packet lengths, the complexity is shown for two different packet lengths. The complexity of the DFT-based demodulator for $N=64$ and a packet length of 250 symbols (which is in the same order as the packet lengths in UNB applications) is almost 150 times the DDPSK complexity. However, DDPSK for $N=64$ leads to a $3 d B$ loss compared to the DFT-Based demodulator and more than $4 d B$ loss compared to DDPSK with $F T=1$.

The complexity of the demodulator refers to the digital complexity (which is more affordable in new technology nodes due to low power consumption) and the energy loss in the BER performance refers to the transmission power. The former puts pressure on the receiver while the latter increases transmitted power consumption. Assessing the trade-off and choosing the best method between the 


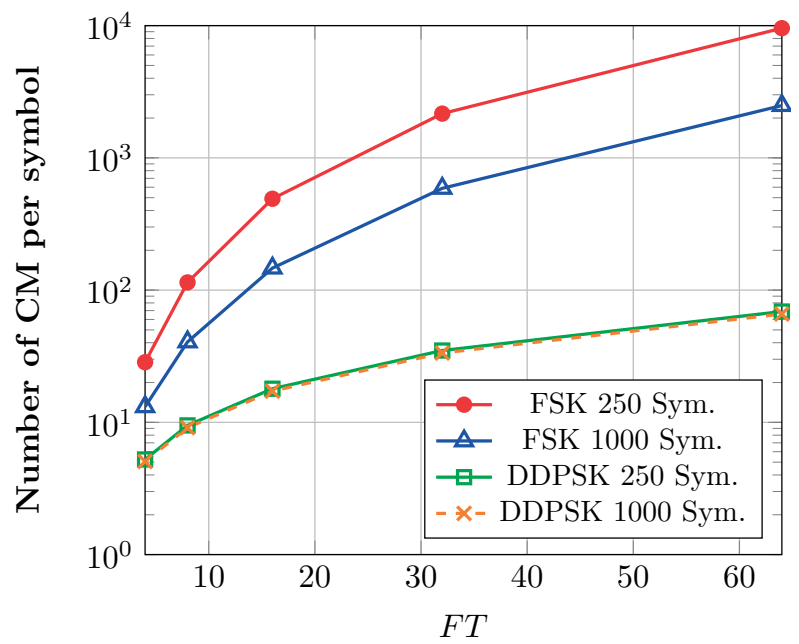

Figure 3.15: The number of complex multiplications required for calculating each symbol

DDPSK and DFT-Based demodulator, needs to take various parameters of the specific target application into account and can be decided at design time.

FSK and PSK both are used in UNB applications and the behavior of the investigated demodulators shows that they are two extreme cases; large BER performance degradation (PSK) and significant complexity (FSK). This raises an interesting question: is it possible to modify these two demodulators such that their limitations become milder and both can be efficiently used if required? This means finding a less complex design for the DFT-Based demodulator (with minor or no sacrifice in the BER performance) and improving the BER performance of DDPSK compared to what is achievable now. These questions are answered in the next two chapters by proposing two different techniques. One alleviates the $E_{b} / N_{0}$ loss in DDPSK when a wide filter is used and the other focuses on an efficient implementation of the DFT-Based demodulator.

\subsection{CONCLUSION}

This chapter investigated two offset tolerant demodulators for FSK and PSK which are capable of tolerating a large frequency offset (multiple times the symbol rate). For FSK, a DFT-based demodulator was designed using a simple detection method. To synchronize the DFT window in the presence of a large frequency offset, an existing DFT-based window synchronization was utilized. For PSK, a Double Differential scheme (DDPSK) and an autocorrelation based demodulator (ACD) were considered. To ensure a fair complexity comparison between these two demodulators, a synchronization scheme for the DDPSK was 
considered as well. These demodulators with their synchronization schemes were simulated for different filter bandwidths and their BER performance and complexity were compared. It was seen that the DFT-based demodulator is robust against increased noise bandwidth due to a wider filter while imposing considerable complexity. In contrast, DDPSK provides a low complexity solution which is sensitive to the filter bandwidth and has significant BER performance degradation in case of large filters (more than $2 \mathrm{~dB}$ loss when $F T$ changes from 1 to 16). In the next two chapters, two solutions are proposed to overcome these limitations of the DFT-Based demodulator and DDPSK. 


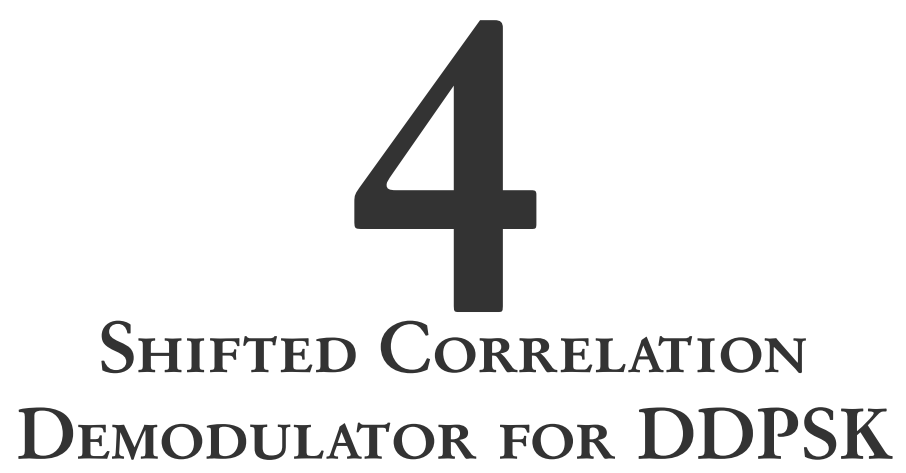

\begin{abstract}
The autocorrelation based demodulator (ACD) for DDPSK is a demodulator which can tolerate frequency offset. However, to tolerate a large frequency offset, the bandwidth of the low-pass filter before the demodulator must increase. It was shown in the previous chapter that this increased filter bandwidth leads to a larger noise bandwidth and degrades BER performance. In this chapter, a new method is proposed to overcome this problem. Instead of one path of ACD, samples at the output of the filter go through multiple paths with adjusted delay and summation blocks in the ACD. The sum of the outputs of these paths provide the input to the detector with an increased SNR compared to the conventional structure. Using the proposed method, the $E_{b} / N_{0}$ required for a specific $B E R$ remains independent of the filter bandwidth if the target BER is less than 0.01 .
\end{abstract}

\title{
4.1 INTRODUCTION
}

The autocorrelation based demodulator (ACD) for second-order differential PSK also known as Double Differential PSK (DDPSK) has been introduced as a demodulator which can detect phase information independent of frequency offset [93]. As explained in the previous chapter, when using an offset tolerant demodulator, the bandwidth of the filter before the demodulator should increase in accordance with the desired range of the frequency offset tolerance. On the other hand, as the noise bandwidth is proportional to the filter bandwidth, the BER performance deteriorates when filter bandwidth increases [94].

In our search for demodulator scalability in terms of frequency offset tolerance, a novel method is proposed in this chapter. The proposed method can overcome

This chapter is based on [S:4]. 
the effect of the increased noise bandwidth when widening the filter prior to the DDPSK demodulator. As a result, increasing the range of offset tolerance does not lead to BER performance degradation which is the case for the conventional method. In addition to simulations, a mathematical approach is followed to analyze noise components and to prove effectiveness of the proposed method.

In the next section, the proposed demodulators for DDPSK are briefly reviewed. Before explicating the proposed method, a conventional ACD for DDPSK is investigated in section 4.3 to achieve a clear view on the reason of the BER performance degradation. This overview takes us to section 4.4 and 4.5 which elaborate on the proposed method and its BER performance, respectively. Simulation results are presented in section 4.6. Although the method improves the BER performance, it adds complexity. The trade-off between BER performance and complexity is investigated in section 4.7 and concluding remarks are drawn in the last part of this chapter, section 4.8 .

\subsection{Related Work}

The ACD for DDPSK can tolerate frequency offset at the expense of BER performance degradation compared to BPSK and DPSK. Different methods have been proposed to improve the performance of DDPSK and make its BER closer to DPSK or BPSK. A Generalized Likelihood detector and Maximum a Posteriori (MAP) detector for DDPSK are investigated in [98, 99]. It is shown that these methods are identical to the simple heuristic detector (which is the same detection scheme used in the autocorrelation based demodulator in the previous chapter). In [84] a variation of a DDPSK demodulator is introduced where the integrator is prior to the differential detection. Moreover, the differential detection is replaced with a multiple symbol sequence detection. Although this method improves the BER performance, it only works when the frequency offset is less than $25 \%$ of the data rate.

In [94], the second stage of the DDPSK after the integrator is replaced with a multiple symbol detector for DPSK. In [12], a Maxmimum Likelihood metric is achieved for multiple symbol detecton of DDPSK which is independent of the frequency offset. To get close to the DPSK performance, these methods considerably increase complexity. As a simpler solution, a Decision-Feedback based detection is proposed in [49] which uses the previously detected symbols to improve the demodulator performance. Using proper weighting factors in the feedback loops, a BER performance close to coherent BPSK can be achieved.

All of the above methods bridge the gap in the performance of DDPSK and BPSK to some extent; however, all of them will experience a performance loss in case of a large frequency offset and wider filter. This loss is not due to the variable phase (as it is solved with all of these methods) but as a consequence of the increased noise bandwidth. Methods in [12, 49], mentioned above, are affected much worse than a conventional ACD by a wide filter. These methods rely on a 
single sample representation of the signal. The single sample representation with matched filter is only valid if the frequency offset is very small. If the bandwidth of the low-pass filter prior to the demodulator increases, the noise variance of the sample increases proportional to the bandwidth of the filter.

For example, with the method proposed in [12], the BER of DDPSK becomes almost equal to the BER of the coherent BPSK with the same SNR (of course with much higher complexity by increasing the observation length). The BER curve of the coherent BPSK is actually the lower bound for the samples with that SNR. However, when a wider filter is used, the $E_{b} / N_{0}$ required to have the same SNR at the demodulator input is increased proportionally to the filter bandwidth. Thus, increasing the filter bandwidth by a factor of two leads to $3 \mathrm{~dB}$ SNR degradation for the same $E_{b} / N_{0}$. As a consequence, the mentioned lower bound will be shifted by $3 \mathrm{~dB}$ making it almost the same as DDPSK [12]. Therefore, although these methods are effective in case of a filter bandwidth equal or close to the signal bandwidth (i.e. frequency offset less than the symbol rate) their performance degrades drastically when the filter becomes wider.

In contrast, the conventional autocorrelation based demodulator (ACD) [93, 94] does not assume a single sample representation. In ACD for DDPSK we can use more than one sample (the number of samples per symbol depends on the bandwidth of the filter). Then, the final filtering is actually the integration which is done after the first stage of autocorrelation. This filter (integrator) is matched to the signal (if a rectangular pulse is used) as the samples have zero frequency after the first autocorrelation stage. As a result, a conventional ACD for DDPSK faces less drastic performance loss when the filter bandwidth increases. However, it still performs poorly when the filter bandwidth becomes several times the symbol rate and it needs to be modified for tolerating large frequency offset. In the next section, we focus on distribution of noise components in the outputs of the first and the second stage of the ACD. The problem is defined based on this understanding which helps us to explain our proposed method.

\subsection{Problem Statement}

The block diagrams of a double differential encoder and an autocorrelation based demodulator (ACD) for Double Differential PSK with a low-pass filter prior to the demodulator are shown in Figure4.1 (at the top and the bottom of the figure, respectively). This is the same as what was introduced in Chapter 3. The double differential encoder puts the information in the double difference of the transmitted symbol phase. The received samples of the double differentially encoded signal are filtered and pass through two consecutive differential decoders. The first stage of the ACD converts the frequency offset to a constant phase offset by correlating each symbol with the previous symbol. Subsequently, the second stage removes the remaining phase offset [93]. Thus, the effect of frequency offset is removed from the final decision variable $d_{n}$. 

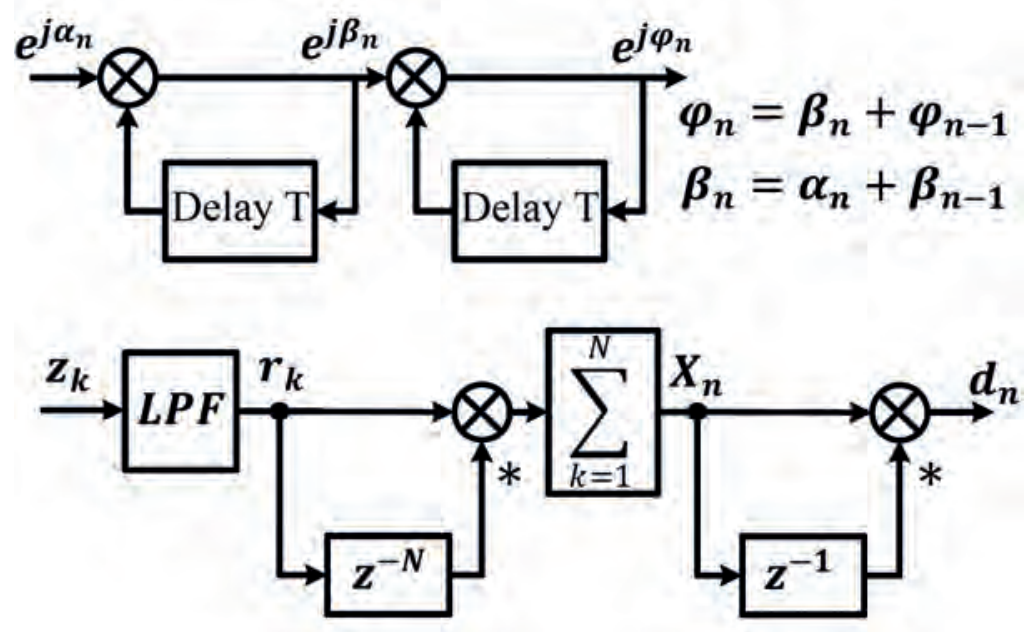

Figure 4.1: Block diagram of a double differential encoder with phase relations and an ACD for DDPSK, $\mathrm{T}$ is the symbol period, $\mathrm{N}$ is the number of samples per symbol and $(*)$ denotes complex conjugate.

As mentioned in Chapter 3, a simple modification to DDPSK, which is achieved without additional complexity, was introduced in [94]. It is obtained by increasing the delay of the second stage of the encoder and the first stage of the demodulator in Figure 4.1 by one symbol period (changing $T$ to $2 T$ and $z^{-N}$ to $z^{-2 N}$, respectively). In the following calculations and the proposed model, a single delay element and $z^{-N}$ is used (the $T-T$ method) for the simplicity of presentation. However, it can be applied to the model with double delay and $z^{-2 N}$ (the $T-2 T$ method) by only changing the delay values accordingly.

Considering the discussion in Chapter 3, it is assumed that the signal passes through a low-pass filter with a bandwidth of $B_{F}$ before the demodulator. The minimum sampling rate after the filter must be $F=2 B_{F}$ (according to the Nyquist theorem) for complex samples. Similar to [94], $F$ is assumed to be equal to an integer multiple of the symbol rate and we use its value normalized to the symbol rate $F T$ (where $T$ is the symbol period). Thus, there are $F T=N$ complex samples per symbol after the filter and at the input of the ACD. Considering an AWGN channel and a filter bandwidth $B_{F}=F / 2$, such a sampling frequency ensures that noise samples are uncorrelated for all $N$ samples per symbol. In case of zero frequency offset, LPF can be replaced with a matched filter to maximize the SNR before the demodulator and one sample per symbol is sent to the demodulator (which will be two consecutive differential decoders). This case is shown by $F T=1$ and has the best BER performance. In the presence of a large frequency offset, a wider filter is required so $F>>1 / T$.

The baseband representation of the samples of a phase modulated signal (PSK, 
DPSK or DDPSK) at the input of the demodulator (see Figure 4.1) during the $n^{\text {th }}$ symbol is:

$$
r_{k, n}=s_{k, n} e^{j\left(\omega_{0} k T_{s}+\theta\right)}+\eta_{k, n}
$$

where $r_{k, n}$ is the $k^{t h}$ sample from the $n^{t h} \operatorname{symbol}(k=1, \ldots, N)$. Considering rectangular pulse shaping and neglecting ISI (which is reasonable for large $B_{F}$ ), $s_{k, n}=s_{n}=e^{j \varphi_{n}}$ where $\varphi_{n}=2 \pi i / M, i=1,2, \ldots, M-1$ for MPSK. $\omega_{O}=2 \pi f_{O}$ is the frequency offset, $T_{s}$ is the sampling period and $\theta$ is a constant phase offset. Without loss of generality the signal amplitude is considered to be one to simplify notations. The $\eta_{k, n}$ components for all $k$ are independent circularly symmetric Gaussian random variables with $\sigma^{2}=N \sigma_{1}^{2}$ where $\sigma_{1}^{2}$ is the noise power in case of $F T=1$. In other words, real and imaginary parts of $\eta_{k, n}$ are independent Gaussian random variables $\mathscr{N}\left(0, N \sigma_{1}^{2} / 2\right)$. The factor $N$ in $\sigma^{2}$ is due to the fact that the noise bandwidth when $F T=N$ is $N$ times the noise bandwidth in case of $F T=1$.

In an $\mathrm{ACD}$, the received samples of consecutive symbols are correlated over a symbol period. This provides the output of the first stage of the ACD as follows.

$$
X_{n}=\sum_{k=1}^{N} r_{k, n} r_{k, n-1}^{*}=S_{n}+\left(H_{s}\right)_{n}+\left(H_{\eta}\right)_{n}
$$

where:

$$
\begin{gathered}
S_{n}=\sum_{k=1}^{N} s_{n} s_{n-1}^{*} e^{j \omega_{O} N T_{s}} \\
\left(H_{s}\right)_{n}=\sum_{k=1}^{N}\left(s_{n} \eta_{k, n-1}^{*}+s_{n-1}^{*} \eta_{k, n}\right) \\
\left(H_{\eta}\right)_{n}=\sum_{k=1}^{N} \eta_{k, n} \eta_{k, n-1}^{*}
\end{gathered}
$$

$S_{n}$ is the only signal component and its power is $N^{2}$ (proportional to the magnitude of the signal component). $\left(H_{s}\right)_{n}$ is the sum of signal-by-noise components. Since all noise samples are independent, all the components in the second summation are independent random variables $\mathscr{C} \mathscr{N}\left(0, \sigma^{2}\right)$ or equivalently $\mathscr{C} \mathscr{N}\left(0, N \sigma_{1}^{2}\right)$. Thus, $\left(H_{s}\right)_{n}$ is in total a $\mathscr{C} \mathscr{N}\left(0,2 N \sigma^{2}\right)$ random variable. The ratio of the power of the signal component to the power of $\left(H_{s}\right)_{n}$ (which is part of noise) is equal to $N / 2 \sigma^{2}=1 / 2 \sigma_{1}^{2}$. This ratio does not change with $N$. Thus, it can be concluded that the other noise component i.e. $\left(H_{\eta}\right)_{n}$ is responsible for the performance degradation when $N$ increases. As $\left(H_{\eta}\right)_{n}$ is the multiplication of two Gaussian complex random variables, it is not Gaussian anymore. The details about the distribution of the noise-by-noise component is mathematically explained in section 4.5. The next section elaborates on the proposed demodulator to overcome this $N=F T$-dependent noise term. 


\subsection{The Proposed Demodulator}

According to the previous section the third term of $X_{n}$ in (4.2) is responsible for an increase is the noise power which is dependent on $N$. Assume that more replicas of the final decision variable are generated with correlated signal components and uncorrelated $\left(H_{\eta}\right)$ components. Then, a final decision variable achieved by adding these replicas has a better effective SNR because of the coherent addition of signal components and the incoherent addition of $\left(H_{n}\right)$ components. For this purpose, we start with generating replicas of $X_{n}$ (using the existing samples) for which the signal terms are correlated and terms resulting from noise-by-noise multiplications are uncorrelated. In this section, the concept is explained and, in the next section, the effectiveness of the proposed method is shown mathematically.

Let's start with the first stage of autocorrelation. The basic idea is illustrated in Figure 4.2. The $(n-1)^{t h}$ and the $n^{\text {th }}$ symbols are shown in this figure. Each square shows one sample of a symbol and, in the figure, four samples per symbol are considered. In the first row under the symbols, the calculation of $X_{n}$ as in the conventional demodulator is shown. In this case the samples are aligned in both symbols i.e. $r_{k, n-1}$ is multiplied by $r_{k, n}$. Now consider a one sample increase in the delay of the first stage decoder (shown in the second row). It is equivalent to shifting the samples of the $(n-1)^{t h}$ symbol to the right for one sample with respect to the samples of the $n^{\text {th }}$ symbol and then do the multiplications. This generates $\left.X_{n, p}^{R}\right|_{p=1}$ in Figure 4.2. In the same way, by decreasing the delay by one sample (shifting the $(n-1)^{t h}$ symbol to the left), $\left.X_{n, p}^{L}\right|_{p=1}$ is calculated. It should be noticed that by shifting symbols by one sample, the number of multiplications decreases to $N-1$ and $\left.X_{n, p}^{R}\right|_{p=1}$ and $\left.X_{n, p}^{L}\right|_{p=1}$ are the sum of $N-1$ terms.

Shifting and correlating can be extended to a shift of $p$ samples to the right or left to achieve $X_{n, p}^{R}$ and $X_{n, p}^{L}$, respectively. This is actually increasing and decreasing (respectively for $X_{n, p}^{R}$ and $X_{n, p}^{L}$ ) the delay of $N$ samples in Figure 4.1 while adjusting the limits of the summation block to make sure that each term in the summation corresponds to the same pair of symbols. It is, indeed, adding duplicate paths of correlation between the signal and its delayed versions with adjusted delay and summation blocks. $X_{n, p}^{R}$ and $X_{n, p}^{L}$ can be written as follows.

$$
\begin{gathered}
X_{n, p}^{R}=\sum_{k=p+1}^{N} r_{k, n} r_{k-p, n-1}^{*}=S_{n, p}^{R}+\left(H_{s}\right)_{n, p}^{R}+\left(H_{\eta}\right)_{n, p}^{R} \\
X_{n, p}^{L}=\sum_{k=1}^{N-p} r_{k, n} r_{k+p, n-1}^{*}=S_{n, p}^{L}+\left(H_{s}\right)_{n, p}^{L}+\left(H_{\eta}\right)_{n, p}^{L}
\end{gathered}
$$



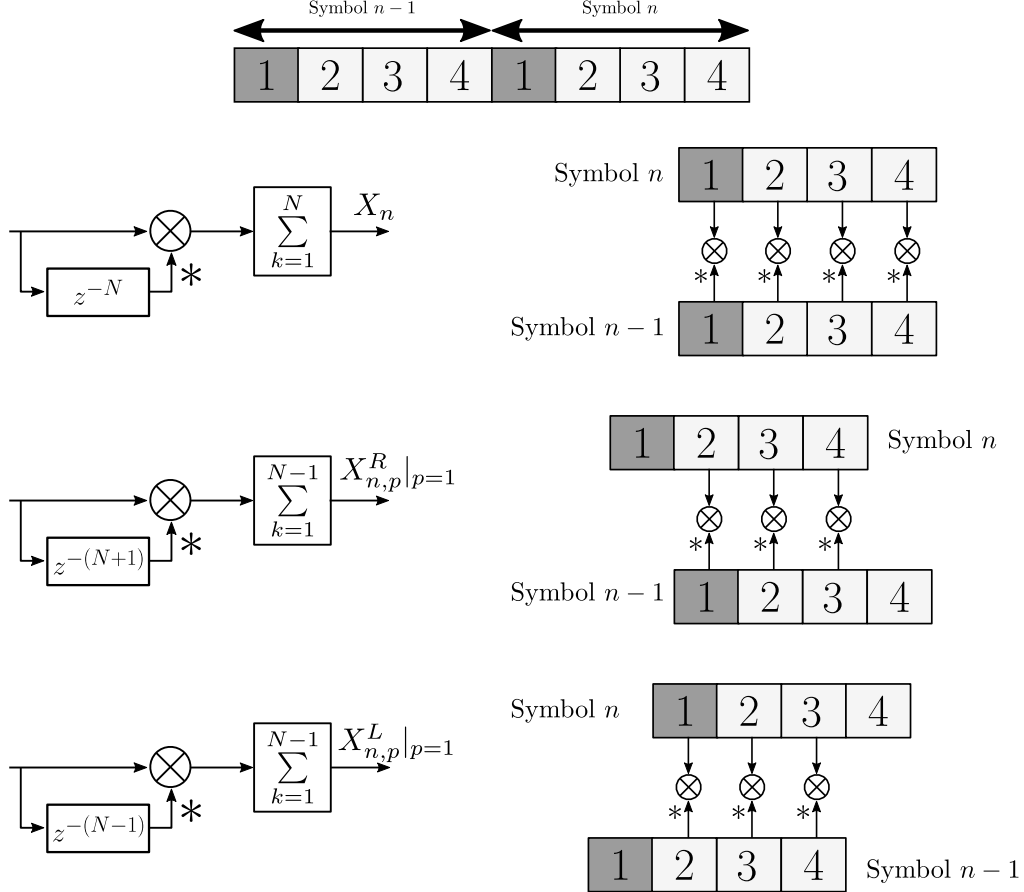

Figure 4.2: The concept of the first stage in the proposed demodulator

where $S_{n, p}^{R}$ and $S_{n, p}^{L}$ are the signal components; $\left(H_{s}\right)_{n, p}^{R}$ and $\left(H_{s}\right)_{n, p}^{R}$ are the noiseby-signal components and $\left(H_{\eta}\right)_{n, p}^{R}$ and $\left(H_{\eta}\right)_{n, p}^{L}$ are the noise-by-noise components as defined in the following equations.

$$
\begin{gathered}
S_{n, p}^{R}=\sum_{k=p+1}^{N} s_{k, n} e^{j\left(\omega k T_{s}+\theta\right)} s_{k-p, n-1}^{*} e^{-j\left(\omega\left(k T_{s}-(N+p) T_{s}\right)+\theta\right)} \\
S_{n, p}^{L}=\sum_{k=1}^{N-p} s_{k, n} e^{j\left(\omega k T_{s}+\theta\right)} s_{k+p, n-1}^{*} e^{-j\left(\omega\left(k T_{s}-(N-p) T_{s}\right)+\theta\right)} \\
\left(H_{s}\right)_{n, p}^{R}=\sum_{k=p+1}^{N}\left(s_{k, n} \eta_{k-p, n-1}^{*}+s_{k-p, n-1}^{*} \eta_{k, n}\right) \\
\left(H_{s}\right)_{n, p}^{L}=\sum_{k=1}^{N-p}\left(s_{k, n} \eta_{k+p, n-1}^{*}+s_{k+p, n-1}^{*} \eta_{k, n}\right) \\
\left(H_{\eta}\right)_{n, p}^{R}=\sum_{k=p+1}^{N} \eta_{k, n} \eta_{k-p, n-1}^{*}
\end{gathered}
$$




$$
\left(H_{\eta}\right)_{n, p}^{L}=\sum_{k=1}^{N-p} \eta_{k, n} \eta_{k+p, n-1}^{*}
$$

The terms $S_{n, p}^{R}$ and $S_{n, p}^{L}$ for all $p$ are correlated. In the previous section, we showed that the ratio of the power of the signal component to the power of $\left(H_{s}\right)_{n}$ is independent of $N$; thus, let us just focus on $\left(H_{\eta}\right)_{n, p}^{R}$ and $\left(H_{\eta}\right)_{n, p}^{L}$ here $\left(\left(H_{s}\right)_{n, p}^{L}\right.$ and $\left(H_{s}\right)_{n, p}^{R}$ will be discussed in the next section). Each term constituting $\left(H_{\eta}\right)_{n, p}^{R}$ and $\left(H_{\eta}\right)_{n, p}^{L}$ is a multiplication of two different and independent Gaussian complex random variables. These new random variables are independent and a detailed discussion about their distribution follows in the next section.

For now, it can be seen that the shifted correlation outputs $\left(X_{n, p}^{R}\right.$ and $\left.X_{n, p}^{L}\right)$ provide us with more replicas of $X_{n}$ with correlated signal components and independent noise-by-noise components. Hence, adding $X_{n, p}^{R}$ and $X_{n, p}^{L}$ for all $p$ can lead to a decision metric with higher effective SNR. There is only one issue left that must be taken into account.

Assuming a signal model similar to (4.1), $S_{n, p}^{R}$ and $S_{n, p}^{L}$ are as follows.

$$
\begin{gathered}
S_{n, p}^{R}=\sum_{k=p+1}^{N} e^{j\left(\Delta \varphi_{n}\right)} e^{j\left(\omega_{O}(N+p) T_{s}\right)} \\
S_{n, p}^{L}=\sum_{k=1}^{N-p} e^{j\left(\Delta \varphi_{n}\right)} e^{j\left(\omega_{O}(N-p) T_{s}\right)}
\end{gathered}
$$

where $\Delta \varphi_{n}=\varphi_{n}-\varphi_{n-1}$ and $T_{s}$ is the sampling period. As can be seen, the constant phase offset is removed but there is still a phase offset at the output of the first stage $\left(\omega_{O}(N \pm p) T_{s}\right)$ which depends on $\omega_{O}$, $p$ and the direction of the $\operatorname{shift}(R$ or $L)$.

If $\omega_{O}=0$, the phases of all signal components are equal to $\Delta \varphi_{n}$. Thus, all $X_{n, p}^{R(L)}$ (which means $X_{n, p}^{R}$ or $X_{n, p}^{L}$ ) can be added to make a decision variable to detect $\Delta \varphi_{n}$. This will work if a DPSK is used and is capable of achieving an effective SNR equal to the SNR in case of $F T=1$ (this will be shown in section 4.5). Improved SNR assures a BER performance practically independent of the filter bandwidth.

If $\omega_{O} \neq 0$, since this constant phase term $\left(\omega_{O}(N \pm p) T_{s}\right)$ depends on $p$, it is not possible to simply add all $X_{n, p}^{R(L)}$ and send them to the next stage of the ACD. Therefore, for each $p$ and direction of shift, the shifted correlation of the symbols $n$ and $n-1\left(X_{n, p}^{R(L)}\right)$ must be first correlated with the shifted correlation of the symbols $n-1$ and $n-2\left(X_{n-1, p}^{R(L)}\right)$. This second stage is visualized in Figure $4 \cdot 3$. 


\begin{tabular}{|c|c|c|c|c|c|c|c|c|c|c|}
\hline \multicolumn{4}{|c|}{ Symbol $n-2$} & \multicolumn{3}{|c|}{ Symbol $n-1$} & \multicolumn{4}{|c|}{ Symbol $n$} \\
\hline 1 & 2 & 3 & 4 & 1 & & 4 & 1 & 2 & 3 & 4 \\
\hline
\end{tabular}
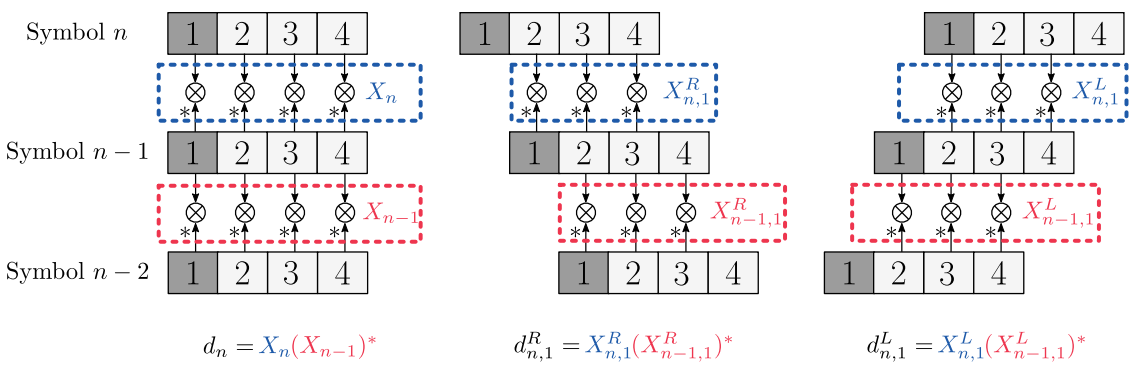

Figure 4.3: The concept of the second stage in the proposed demodulator

The decision variable for each path, $d_{n, p}^{R(L)}$ is as follows.

$$
d_{n, p}^{R(L)}=S_{n, p}^{R(L)} S_{n-1, p}^{R(L)}+H_{n o i s e}
$$

where $H_{\text {noise }}$ represents all noise terms.

Considering $d_{n, p}^{R}$, the signal component is:

$$
\begin{aligned}
S_{n, p}^{R}\left(S_{n-1, p}^{R}\right)^{*} & =\left(\sum_{k=p+1}^{N} e^{j\left(\Delta \varphi_{n}\right)} e^{j\left(\omega_{O}(N+p) T_{s}\right)}\right)\left(\sum_{k=p+1}^{N} e^{j\left(\Delta \varphi_{n-1}\right)} e^{j\left(\omega_{O}(N+p) T_{s}\right)}\right)^{*} \\
& =(N-p)^{2} e^{j\left(\Delta \varphi_{n}-\Delta \varphi_{n-1}\right)}
\end{aligned}
$$

As can be seen, the phase of the signal component in $d_{n, p}^{R(L)}$ is only determined by $\left(\Delta \varphi_{n}-\Delta \varphi_{n-1}\right)$ which is the same phase difference used in the conventional ACD for DDPSK. Therefore, it is possible to add all $d_{n, p}^{R(L)}$ and make the decision based on $d_{n, \text { Total }}$ which is defined as follows.

$$
d_{n, \text { Total }}=d_{n}+\sum_{p=1}^{N-1}\left(d_{n, p}^{R}+d_{n, p}^{L}\right)
$$

where $d_{n}$ is the decision variable achieved by the path equivalent to the conventional demodulator. The block diagram of a demodulator based on our discussions is demonstrated in Figure 4.4. The overlaying rectangles show the duplicate paths for different $p$ values. In the next section, the error probability is investigated and the effectiveness of the proposed demodulator is shown mathematically. 


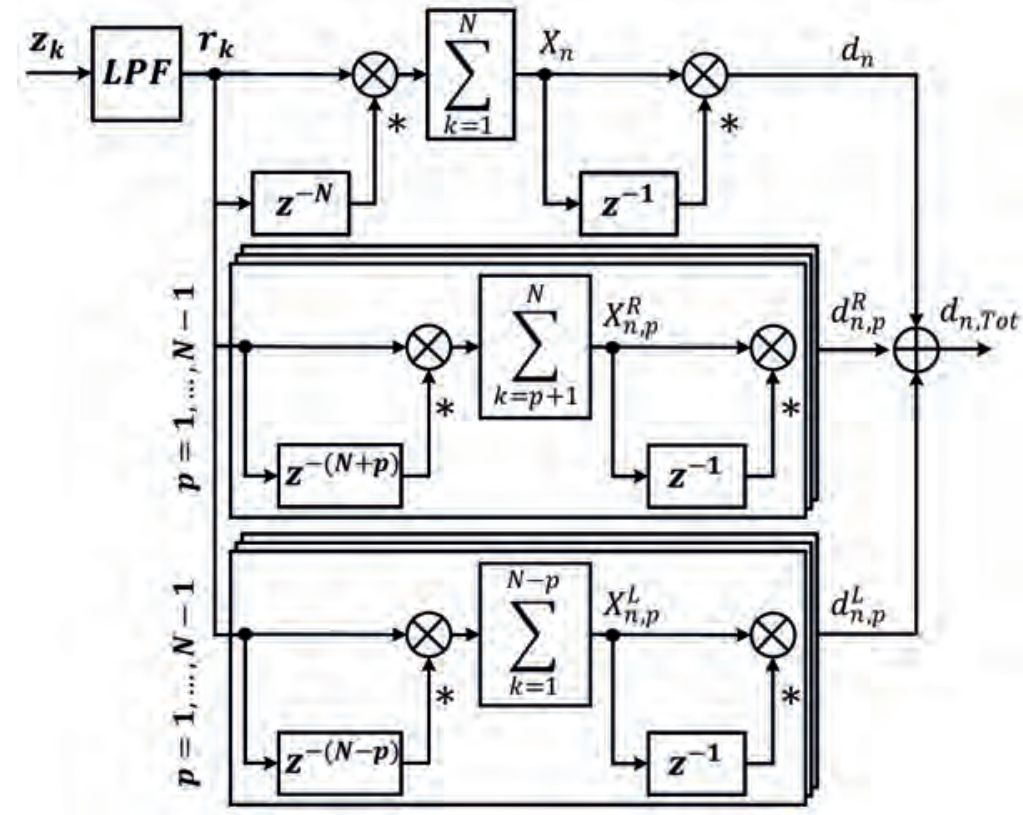

Figure 4.4: Block diagram of the proposed demodulator for DDPSK

\subsection{Error Probability}

The proposed method is designed for DDPSK and it removes the effect of the wide filter particularly by improving the first stage of the ACD. As mentioned earlier, the single-stage ACD can be used for demodulating a DPSK signal when $\omega_{O}=0$. Although our main target is DDPSK, we also consider single-stage ACD for DPSK in the analysis of the proposed method. It helps to demonstrate how the proposed method affects the SNR after the first stage of the ACD. For simpler representation, the frequency and phase offset are not included in the signal models in this section. This does not violate the generality of the formulations because those components do not affect the power of the signal and noise.

\subsubsection{Single-Stage ACD}

For a single stage $\mathrm{ACD}$, all $X_{n, p}^{R(L)}\left(X_{n, p}^{R}\right.$ or $X_{n, p}^{L}$ ) values and $X_{n}$ (the output of the path without shifting) in Figure4.2 are added directly to form $X_{n, T o t}$.

$$
X_{n, T o t}=X_{n}+\sum_{p=1}^{N-1}\left(X_{n, p}^{R}+X_{n, p}^{L}\right)
$$

It is difficult (if not impossible) to derive a closed form expression for the error 
probability of the proposed method. Therefore, to prove its effectiveness, an alternative approach is adopted. The signal and noise components of $X_{n, T o t}$ are modeled. Then, the ratio of the signal power to the power of each noise component is calculated for $F T=1$ and $F T=N$. As the distributions of the noise components are the same in both cases (it is shown in the sequel), equal signal to noise ratio is equivalent to similar error probability. First, the two basic assumptions are repeated:

(I). The sampling frequency equals $F$ for each real and imaginary part $(F T=$ $N$ complex samples per symbol). This means that all noise samples $\left(\eta_{k, n}\right)$ are independent circularly symmetric Gaussian noise $\mathscr{C} \mathscr{N}\left(0, \sigma^{2}\right)$ i.e. $\operatorname{Re}\left\{\eta_{k, n}\right\}$ and $\operatorname{Im}\left\{\eta_{k, n}\right\}$ are independent Gaussian random variables $N\left(0, \sigma^{2} / 2\right)$.

(II). Rectangular pulse shaping is assumed and ISI is ignored which is reasonable when $F T$ is large. So the signal component of all samples in a symbol is the same.

Using the signal model (4.1) and replacing terms within (4.19) with their expansions, after some mathematical manipulations we have:

$$
X_{n, T o t}=S_{T o t}+\left(H_{s}\right)_{T o t}+\left(H_{\eta}\right)_{T o t}
$$

where $S_{T o t}$ is the signal component while $\left(H_{s}\right)_{T o t}$ and $\left(H_{\eta}\right)_{T o t}$ are two noise components resulting from signal-by-noise and noise-by-noise multiplications, respectively. These three components in (4.20) are as follows:

$$
\begin{aligned}
S_{T o t}= & \sum_{k=1}^{N} s_{k, n} s_{k, n-1}^{*} \\
& +\sum_{p=1}^{N-1}\left(\sum_{k=p+1}^{N} s_{k, n} s_{k-p, n-1}^{*}+\sum_{k=1}^{N-p} s_{k, n} s_{k+p, n-1}^{*}\right) \\
= & N^{2} s_{n} s_{n-1}^{*} \\
\left(H_{s}\right)_{T o t}= & \sum_{k=1}^{N}\left(s_{k, n} \eta_{k, n-1}^{*}+s_{k, n-1}^{*} \eta_{k, n}\right) \\
& +\sum_{p=1}^{N-1} \sum_{k=p+1}^{N}\left(s_{k, n} \eta_{k-p, n-1}^{*}+s_{k-p, n-1}^{*} \eta_{k, n}\right) \\
& +\sum_{p=1}^{N-1} \sum_{k=1}^{N-p}\left(s_{k, n} \eta_{k+p, n-1}^{*}+s_{k+p, n-1}^{*} \eta_{k, n}\right) \\
= & \sum_{k=1}^{N} N s_{n-1}^{*} \eta_{k, n}+\sum_{k=1}^{N} N s_{n} \eta_{k, n-1}^{*}
\end{aligned}
$$




$$
\left(H_{\eta}\right)_{T o t}=\sum_{k=1}^{N} \eta_{k, n} \eta_{k, n-1}^{*}+\sum_{p=1}^{N-1} \sum_{k=p+1}^{N} \eta_{k, n} \eta_{k-p, n-1}^{*}
$$

$$
\begin{aligned}
& +\sum_{p=1}^{N-1} \sum_{k=1}^{N-p} \eta_{k, n} \eta_{k+p, n-1}^{*} \\
= & \sum_{k=1}^{N} \sum_{i=1}^{N} \eta_{i, n} \eta_{k, n-1}^{*}
\end{aligned}
$$

As a result of assumption (I), all individual terms within (4.22) i.e. $N s_{n-1}^{*} \eta_{k, n}$ and $N s_{n} \eta_{k, n-1}^{*}$ are independent circularly symmetric Gaussian random variables $\mathscr{C} \mathscr{N}\left(0, N^{2} \sigma^{2}\right)$; thus:

$$
\left(H_{s}\right)_{T o t}=\mathscr{C} \mathscr{N}\left(0,2 N^{3} \sigma^{2}\right)
$$

$\operatorname{In}\left(H_{\eta}\right)_{T o t}$, all terms $\left(\eta_{i, n} \eta_{k, n-1}^{*}\right)$ are also independent complex random variables where the real and imaginary parts have the same distribution. First we discuss the distribution of the real part. In $\operatorname{Re}\left\{\left(H_{\eta}\right)_{T o t}\right\}$ each component can be written as follows:

$$
\operatorname{Re}\left\{\eta_{i, n} \eta_{k, n-1}^{*}\right\}=\eta_{i, n}^{R e} \eta_{k, n-1}^{R e}+\eta_{i, n}^{I m} \eta_{k, n-1}^{I m}
$$

where the superscripts $R e$ and $I m$ denote real part and imaginary part, respectively. $\eta_{i, n}^{R e}, \eta_{k, n-1}^{R e}, \eta_{i, n}^{I m}$ and $\eta_{k, n-1}^{I m}$ are independent Gaussian random variables $\left(\mathscr{N}\left(0, \sigma^{2} / 2\right)\right)$ according to assumption (I).

To obtain the distribution of the product of two independent Gaussian random variables $x$ and $y$ with zero mean and similar variance $\left(\sigma_{x}^{2}\right), x y$ can be written as $x y=(x+y)^{2} / 4-(x-y)^{2} / 4$ where $x+y$ and $x-y$ are independent Gaussian variables with zero mean and $2 \sigma_{x}^{2}$ variance. The square of such a Gaussian random variable has a gamma distribution with shape and rate parameters equal to $1 / 2$ and $1 / \sigma_{x}^{2}$, respectively $\left(\Gamma\left(1 / 2,1 / \sigma_{x}^{2}\right)\right)[54]$. Thus, the two terms of $x y$ are independent gamma distributed random variables $\Gamma\left(1 / 2,4 / \sigma_{x}^{2}\right)$. Moreover, the sum of two gamma random variables, $\Gamma\left(\alpha_{1}, \beta\right)$ and $\Gamma\left(\alpha_{2}, \beta\right)$, is a gamma random variable with shape parameter $\alpha 1+\alpha 2$ and rate parameter $\beta,(\Gamma(\alpha 1+\alpha 2, \beta))$ [54]. Considering the distribution of $\eta_{i, n}$ and $\eta_{k, n-1}^{*}$ (which is $\mathscr{C} \mathscr{N}\left(0, \sigma^{2}\right)$ ) and the above calculations, $\operatorname{Re}\left\{\eta_{i, n} \eta_{k, n-1}^{*}\right\}$ is the difference of two independent random variables with a $\Gamma\left(1,8 / \sigma^{2}\right)$ distribution. Hence, $\operatorname{Re}\left\{\left(H_{\eta}\right)_{T o t}\right\}$ is obtained as:

$$
\operatorname{Re}\left\{\left(H_{\eta}\right)_{T o t}\right\}=\sum_{i=1}^{N^{2}} R_{i}-\sum_{i=1}^{N^{2}} Q_{i}
$$

where $R_{i}$ and $Q_{i}$ are independent random variables with gamma distribution $\left(R_{i}, Q_{i} \sim \Gamma\left(1,8 / \sigma^{2}\right)\right)$. Consequently, the distribution of $\operatorname{Re}\left\{\left(H_{\eta}\right)_{T o t}\right\}$ will be equal to difference of two independent random variables with a $\Gamma\left(N^{2}, 8 / \sigma^{2}\right)$ distribution. 
Subtracting two independent gamma random variables $\Gamma(1 / \lambda, 1 / \sqrt{\lambda \theta})$ results in a random variable with Double Gamma Difference $(D G D(\lambda, \theta))$ distribution with a variance of $2 \theta$ [9]. So, the overall distribution of $\operatorname{Re}\left\{\left(H_{\eta}\right)_{\text {Tot }}\right\}$ is a Double Gamma Difference distribution, $D G D\left(1 / N^{2}, N^{2} \sigma^{4} / 64\right)$. The variance (power)

of the noise component with such a distribution is $N^{2} \sigma^{4} / 32$. Doing similar calculations, $\operatorname{Im}\left\{\left(H_{\eta}\right)_{T o t}\right\}$ also has a $D G D\left(1 / N^{2}, N^{2} \sigma^{4} / 64\right)$ distribution. Since the real and the imaginary parts are independent, the total power of $\left(H_{\eta}\right)_{T o t}$ is $N^{2} \sigma^{4} / 16$. Following the same procedure, the power of the noise components for $F T=1$ are $2 \sigma_{1}^{2}$ and $\sigma_{1}^{4} / 16$ for $\left(H_{s}\right)_{T o t}$ and $\left(H_{\eta}\right)_{T o t}$, respectively, where $\sigma_{1}^{2}$ is the noise power (at the input of $\mathrm{ACD}$ ) when $F T=1$.

According to (4.21), the power of the signal component in $X_{n, T o t}$ when $F T=N$ is $N^{4}$ times its value in the $F T=1$ case. Besides, it is clear that $\sigma^{2}=N \sigma_{1}^{2}$ (for $F T=N$, the noise spectral density is the same while its bandwidth is multiplied by $N$ ). For convenience in further analysis, we use a specific notation of SNR where the ratio of the power of the signal component to the power of each noise component is considered separately. $S N R_{H_{s}}$ and $S N R_{H_{\eta}}$ represent this ratio for $H_{s}$ and $H_{\eta}$ components, respectively. Taking (4.24) and (4.26) into account, we have the following equations for the SNR corresponding to different noise components in $X_{n, \text { Total }}$ when $F T=N$ :

$$
\begin{gathered}
S N R_{H_{s}}=\frac{N^{4} P_{s, 1}}{2 N^{3}\left(N \sigma_{1}^{2}\right)}=\frac{P_{s, 1}}{2 \sigma_{1}^{2}} \\
S N R_{H_{\eta}}=\frac{N^{4} P_{s, 1}}{N^{2} \sigma^{4} / 16}=\frac{P_{s, 1}}{\sigma_{1}^{4} / 16}
\end{gathered}
$$

where $P_{s, 1}$ is the power of the signal component in $X_{n, T o t}$ when $F T=1$. As can be seen, the SNR corresponding to both noise components $\left(\left(H_{s}\right)_{\text {Tot }}\right.$ and $\left.\left(H_{\eta}\right)_{T o t}\right)$ in the proposed method when $F T=N$ are the same as SNR values in case of $F T=1$. It means that the BER performance for the proposed method is equal for both $F T=1$ and $F T=N$.

To clarify the difference between the proposed method and the conventional method (the method without the shifted correlation), the SNR values corresponding to $H_{s}$ and $H_{\eta}$ in the output of a conventional single-stage ACD with $F T=N$ are calculated. In this case, the output is simply equal to $X_{n}(4.2)$. As mentioned in section $4 \cdot 3$, for $F T=N$, the power of the signal component in $X_{n}$ is $N^{2} P_{s, 1}$ and the power of $\left(H_{s}\right)_{n}$ in $X_{n}$ is $2 N \sigma^{2}$ where $\sigma^{2}=N \sigma_{1}^{2}$ (see explanations following (4.2)). Additionally, considering (4.5) and the above discussion about the noise distributions, $\operatorname{Re}\left(H_{\eta}\right)_{n}$ and $\operatorname{Im}\left(H_{\eta}\right)_{n}$ have $D G D\left(1 / N, N \sigma^{4} / 64\right)$ which leads to a total power of $N \sigma^{4} / 16$ for $\left(H_{\eta}\right)_{n}$ (notice that $\left(H_{\eta}\right)_{n}$ has $N$ components while $\left(H_{\eta}\right)_{T o t}$ has $N^{2}$ components). Therefore, $S N R_{H_{s}}$ and $S N R_{H_{\eta}}$ are achieved as follows. 


$$
S N R_{H_{s}}=\frac{N^{2} P_{s, 1}}{2 N\left(N \sigma_{1}^{2}\right)}=\frac{P_{s, 1}}{2 \sigma_{1}^{2}}
$$

$$
S N R_{H_{\eta}}=\frac{N^{2} P_{s, 1}}{N \sigma^{4} / 16}=\frac{P_{s, 1}}{N \sigma_{1}^{4} / 16}
$$

As shown by (4.30), the ratio of the signal power to the power of $\left(H_{\eta}\right)_{\text {Tot }}$ for the proposed method (see (4.28)) is $N$ times its ratio for the conventional single-stage ACD with only one path, whereas, as clarified by (4.27) and (4.29) this ratio for $\left(H_{s}\right)_{\text {Tot }}$ is the same for the proposed method as for the conventional method. In the proposed method, although the $H_{\eta}$ components are uncorrelated, the individual terms in $H_{s}$ are correlated. As can be seen in (4.29), even for the conventional ACD, $S N R_{H_{s}}$ is equal for both $F T=1$ and $F T=N$. In other words, $H_{\eta}$ (and not $H_{s}$ ) is responsible for the difference in BER performance for various $F T$ values and the proposed method removes the effect of $H_{\eta}$. This confirms our claim in the previous section.

\subsubsection{Two-STAGE ACD}

The output of each path in the proposed demodulator $\left(d_{n, p}^{R(L)}\right.$ in Figure 4.4) is composed of different components including signal-by-signal, signal-by- $H_{s}$, signal-by- $H_{\eta}, H_{s}-$ by- $H_{\eta}$ and $H_{\eta}$-by- $H_{\eta}$. For large SNR, the terms including multiplication of noise components resulting from the first stage $\left(H_{s}-\right.$ by- $H_{\eta}$ and $H_{\eta}$-by- $\left.H_{\eta}\right)$ are negligible; thus, $d_{n, p}^{R}$ and $d_{n, p}^{L}$ can be approximated as follows:

$$
\begin{aligned}
d_{n, p}^{R(L)} \approx & S_{n, p}^{R(L)} S_{n-1, p}^{R(L)} \\
& +S_{n, p}^{R(L)}\left(\left(H_{s}\right)_{n-1, p}^{R(L)}\right)^{*}+\left(S_{n-1, p}^{R(L)}\right)^{*}\left(H_{s}\right)_{n, p}^{R(L)} \\
& +S_{n, p}^{R(L)}\left(\left(H_{\eta}\right)_{n-1, p}^{R(L)}\right)^{*}+\left(S_{n-1, p}^{R(L)}\right)^{*}\left(H_{\eta}\right)_{n, p}^{R(L)}
\end{aligned}
$$

where $S_{n, p}^{R(L)},\left(H_{s}\right)_{n, p}^{R(L)}$ and $\left(H_{\eta}\right)_{n, p}^{R(L)}$ are signal, signal-by-noise and nois-by-noise components of $X_{n, p}^{R(L)}$, respectively. As shown before, the proposed method does not affect $S N R_{H_{s}}$, so only the effect of noise components with $H_{\eta}$ (the third line in $(4 \cdot 31))$ is analyzed here.

According to (4.16), the proposed method detects the transmitted symbol based on $d_{n, \text { Total }}$ which is the sum of $d_{n}$ and $d_{n, p}^{R(L)}$ for all $p$. The rest of the mathematical calculations focus on the sum of $S_{n, p}^{R(L)}\left(\left(H_{\eta}\right)_{n-1, p}^{R(L)}\right)^{*}$ components in $d_{n, \text { Total }}$ for different values of $p$ (in the remainder referred to as $S \times H_{\eta}^{*}$ ) and the same can be derived for $\left(S_{n-1, p}^{R(L)}\right)^{*}\left(H_{\eta}\right)_{n, p}^{R(L)}$. Since $S_{n, p}^{R(L)}=(N-p) s_{n} s_{n-1}^{*} \triangleq(N-p) z_{n}$, 
the corresponding noise component in the demodulator output is:

$$
\begin{aligned}
S \times H_{\eta}^{*}= & N z_{n}\left(H_{\eta}\right)_{n}+\sum_{p=1}^{N-1} z_{n}(N-p)\left(\left(H_{\eta}\right)_{n, p}^{R}\right)^{*} \\
& +\sum_{p=1}^{N-1} z_{n}(N-p)\left(\left(H_{\eta}\right)_{n, p}^{L}\right)^{*}
\end{aligned}
$$

Since all $\eta_{k, n} \eta_{i, n-1}^{*}$ are independent random variables, $\left(H_{\eta}\right)_{n}$ and $\left(\left(H_{\eta}\right)_{n, p}^{R(L)}\right)^{*}$ are complex random variables (DGD distribution for real and imaginary parts) with a variance of $N \sigma^{4} / 16$ and $(N-p) \sigma^{4} / 16$, respectively (this is derived using the same process as in the previous section). As the signal component, $z_{n}$, is a constant complex number, where $z_{n} z_{n}^{*}=1(4.1)$, the variance of each term inside the sum over $p$ in $(4.32)$ would be $(N-p)^{3} \sigma^{4} / 16$ and the variance of the first term on (4.32) is $N^{3} \sigma^{4} / 16$. All terms in the right hand side of (4.32) are independent, so the total power is:

$$
P_{\left(S \times H_{\eta}^{*}\right)}=\frac{\sigma^{4}}{16}\left(N^{3}+\sum_{p=1}^{N-1} 2(N-p)^{3}\right)=\frac{N^{2}\left(N^{2}+1\right)}{2} \frac{\sigma^{4}}{16}
$$

When $F T=N$, the signal component $S_{\left(d_{n}\right)_{T o t}, F T=N}$ in $d_{n, T o t}$ which is the final output of the demodulator (see (4.18)) equals to:

$$
\begin{aligned}
S_{\left(d_{n}\right)_{T o t}, F T=N} & =S_{n}+\sum_{p=1}^{N-1} S_{n, p}^{R}\left(S_{n-1, p}^{R}\right)^{*}+\sum_{p=1}^{N-1} S_{n, p}^{L}\left(S_{n-1, p}^{L}\right)^{*} \\
& =z_{n} z_{n-1}^{*}\left(N^{2}+2 \sum_{p=1}^{N-1}(N-p)^{2}\right) \\
& =z_{n} z_{n-1}^{*}\left(\frac{N\left(2 N^{2}+1\right)}{3}\right)
\end{aligned}
$$

To achieve the last line of (4.34), Faulhaber's formula for the sum of squares of $N$ first positive integers $(1,2, \ldots, N)$ can be used. When $F T=1$ the signal component is $z_{n} z_{n-1}^{*}$ so, according to (4.34), the power of the signal component in the output of the proposed demodulator, when $F T=N$, is $N^{2}\left(2 N^{2}+1\right)^{2} / 9$ times the power of the signal component for $F T=1, P_{s, 1}$. Thus, from (4.33) $S N R_{S \times H_{\eta}^{*}, F T=N}$ is:

$$
S N R_{S \times H_{\eta}^{*}, F T=N}=\frac{32}{9} \frac{N^{2}\left(2 N^{2}+1\right)^{2} P_{s, 1}}{N^{2}\left(N^{2}+1\right) \sigma^{4}}
$$

Based on (4.35), $S N R_{S \times H_{\eta}^{*}, F T=1}(N=1)$ can be derived. 


$$
S N R_{S \times H_{\eta}^{*}, F T=1}=16 \frac{P_{s, 1}}{\sigma_{1}^{4}}
$$

Considering that $\sigma^{2}=N \sigma_{1}^{2}$ and from (4.35) and (4.36), the ratio of the SNR corresponding to the $S \times H_{\eta}^{*}$ component for $F T=N$ and $F T=1$ can be calculated as:

$$
\frac{S N R_{S \times H_{\eta}^{*}, F T=N}}{S N R_{S \times H_{\eta}^{*}, F T=1}}=\frac{8 N^{4}+8 N^{2}+2}{9 N^{4}+9 N^{2}}
$$

The limit of the above ratio when $N \rightarrow \infty$ is 0.89 (only $11 \%$ loss compared to $F T=1$ ) while for $N=2$ this ratio is 0.9 . Using only $X_{n}$ as the output and similar to $(4.30)$, the same ratio (as in (4.37)) for conventional DDPSK will be equal to $1 / N$. Although $S N R_{S \times H_{n}^{*}, F T=N}$ for the proposed method is not equal to the case of $F T=1$, it is much higher than its value for the conventional method. This higher SNR leads to an improved BER performance for the proposed method compared to the conventional DDPSK. Besides, the minimum SNR, related to the discussed noise components for the proposed demodulator, equals 0.89 times its value for $F T=1$ even in case of arbitrarily large $N$.

\subsection{Simulation Results and BER Performance Analysis}

To demonstrate the performance of the multipath ACD demodulator and to verify the mathematical calculations presented in the previous section, the BER curves for DPSK and DDPSK are presented in this section. Figure 4.5 and Figure 4.6 show BER curves for Binary DPSK (DBPSK) and Binary DDPSK (DDBPSK), respectively. Similar to [94], BER curves are presented for different values of $F T$ including $F T=1,8,16$. For comparison, the BER curves for the conventional demodulator are also demonstrated.

As can be seen in Figure 4.5, for $F T=1$ in a conventional ACD, the BER performance is the same as a non-coherent DBPSK; whereas, for $F T=8$ and $F T=16$ the performance degrades considerably. As can be seen, the $E_{b} / N_{0}$ for a $B E R=0.1 \%$ increases by $2 d B$ and $3 d B$ for $F T=8$ and $F T=16$, respectively. Using the proposed structure for a single-stage ACD, the BER curves for both $F T$ values exactly match the BER curve for $F T=1$ which matches the conclusion of our discussion in the previous section.

For DDBPSK, the proposed method is applied to $T-2 T$ DDBPSK and the results are shown for $F T=1,8,16$ in Figure 4.6. The proposed method is applied to the $T-2 T$ DDBPSK by replacing $N$ with $2 N$ in the delays of the first stage of the proposed demodulator (see Figure 4.4). To demonstrate the improvement achieved by the proposed method in case of DDBPSK, the BER curves of a conventional demodulator for $F T=8,16$ are illustrated. It can be seen that for $F T=16$ the proposed method saves $E_{b} / N_{0}$ required for $B E R=0.1 \%$ by almost 


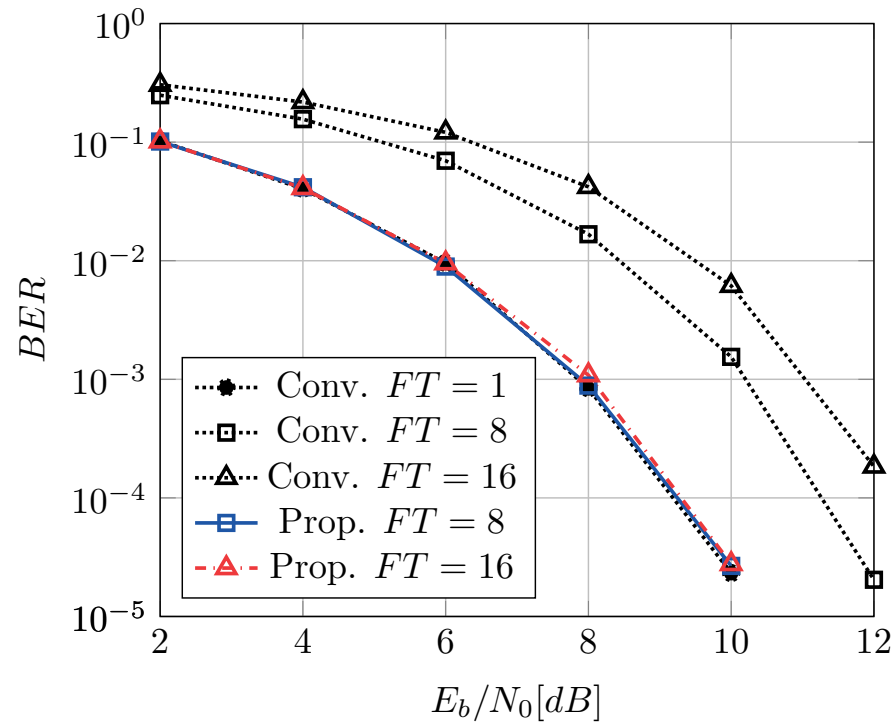

Figure 4.5: BER performance of the proposed method for Differential BPSK and single-stage ACD.

$2 \mathrm{~dB}$. The slight difference in BER performance between $F T=8$ and $F T=16$ in the $E_{b} / N_{0}$ values lower than $9 d B$ is caused by the terms generated by the higher powers of the noise components. These are the noise components neglected in our calculations in the previous section. For higher $E_{b} / N_{0}$, the effect of these values decreases such that the BER curves get closer to that of $F T=1$.

The small deviation from $F T=1$ which remains even for large $E_{b} / N_{0}$ is due to the decrease in the $S N R_{S \times H_{n}^{*}, F T=N}$ (compared to $F T=1$ case), which was shown in the previous section, as well as the higher powers of noise components which were not considered in our calculations.

In Figure 4.7 the proposed method is evaluated for larger values of $F T=N$ by considering the $E_{b} / N_{0}$ required for $B E R=0.1 \%$. For the conventional demodulator, when the filter bandwidth equals 64 times the symbol rate $(F T=$ $N=128$ ), there is $5.5 d B$ loss compared to $F T=1$ which achieves the $B E R=$ $0.1 \%$ with $E_{b} / N_{0}=10 \mathrm{~d} B$. However, the loss for the proposed method is less than $0.5 \mathrm{~dB}$. This small loss is the result of higher order components that were ignored in (4.31). According to the previous chapter, if the transition band of the filter is zero (an ideal filter), FT $=128$ means that the demodulator can tolerate a frequency offset up to around \pm 63 times the symbol rate. 


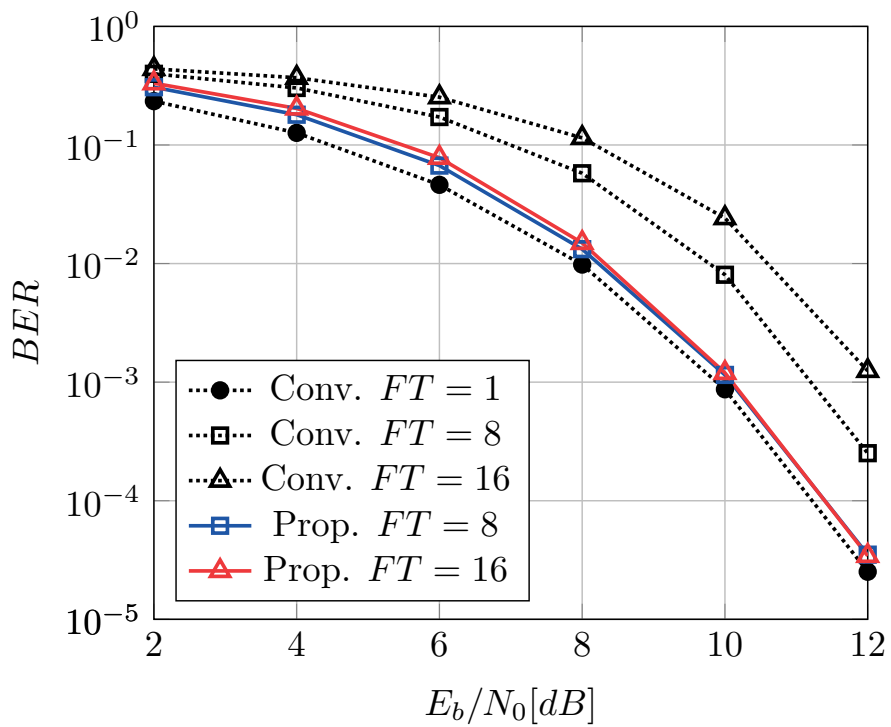

Figure 4.6: BER performance of the proposed method for Double Differential BPSK and two-stage ACD

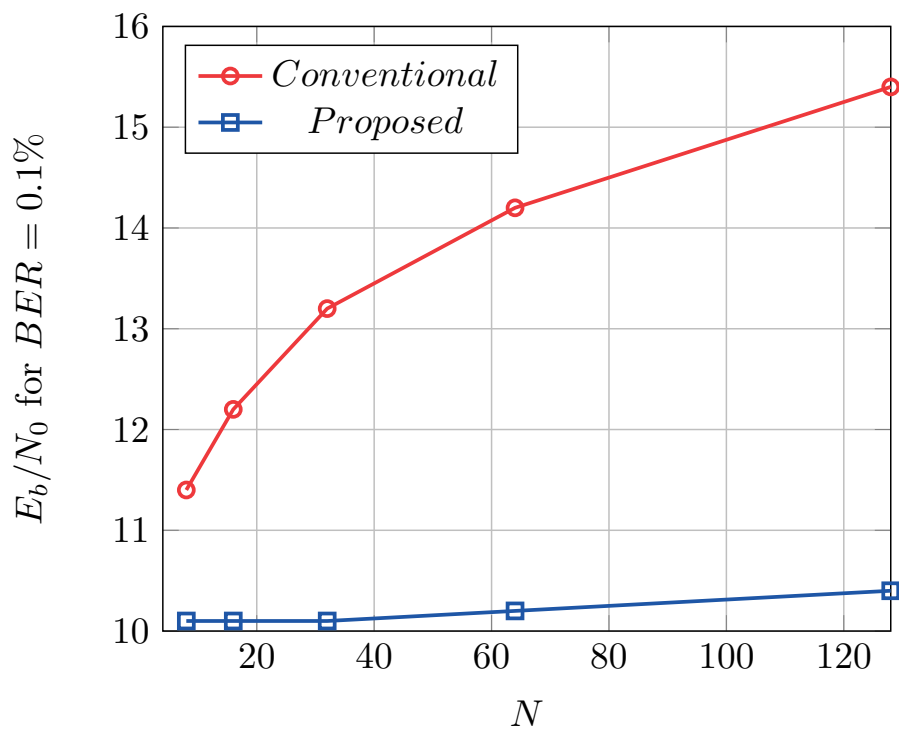

Figure 4.7: The $E_{b} / N_{0}$ required for a $B E R=0.1 \%$ when $N$ increases 


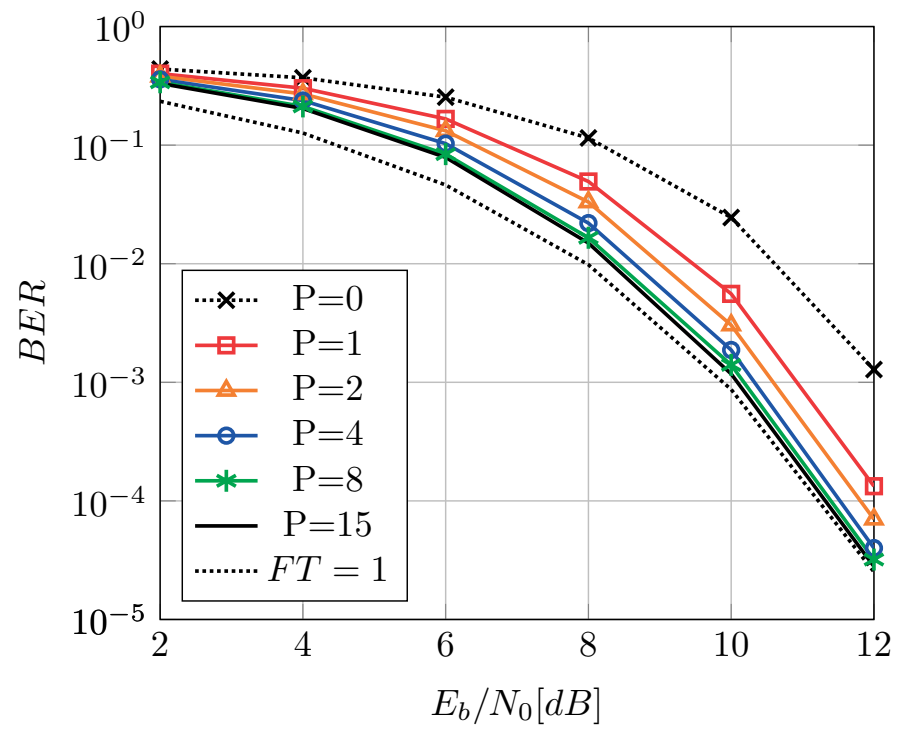

According to the results presented so far, the BER performance of the proposed demodulator remains almost constant when the filter bandwidth increases. Obviously, this improvement is achieved at the cost of increased digital complexity. Inspecting the structure of the proposed demodulator, one may recognize a tradeoff between the complexity and the BER performance. When all paths are used i.e. $p=1, \ldots, N-1$, the BER performance is almost the same as $F T=1$. Nevertheless, all paths do not contribute equally to the performance improvement.

When $p$ increases, the number of samples used for calculating $X_{n, p}^{R(L)}$ decreases due to shifting symbols relative to each other (see Figure 4.2). It means that the $X_{n, p}^{R(L)}$ for higher values of $p$ generates fewer uncorrelated replicas of the noiseby-noise component. If a compromise for the BER performance is acceptable, fewer paths can be used. Each path adds $N-p$ complex multiplications in the first stage and one complex multiplication in the second stage.

To see this effect, the BER curves in case of $F T=16$ and $F T=64$ for different values of $P$ (where $p=1, \ldots, P$ ) are shown in Figure 4.8 and 4.9 , respectively.

These figures show that only adding one path (in each direction) contributes to almost half of the saving in the $E_{b} / N_{0}$ required for $B E R=0.1 \%$. It is interesting that for $P=N / 2$ the BER curve is hardly distinguishable from the best case $(P=N-1)$. However, the complexity for $P=N / 2$ is $75 \%$ of the complexity 


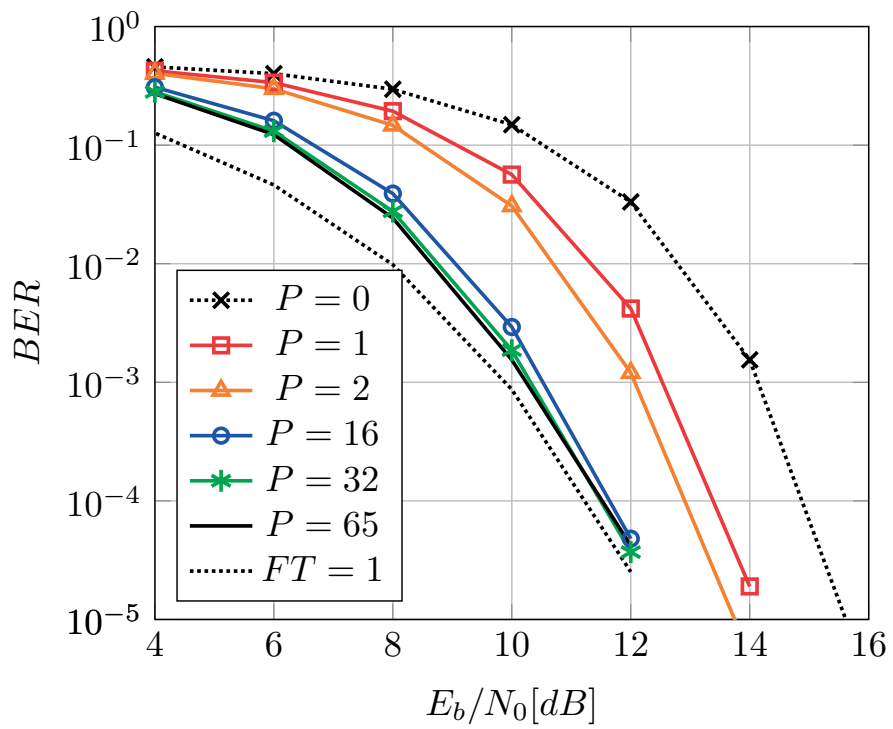

Figure 4.9: The BER performance of the proposed demodulator for different values of $P$ where $p=1, \ldots, P$ and $F T=64$
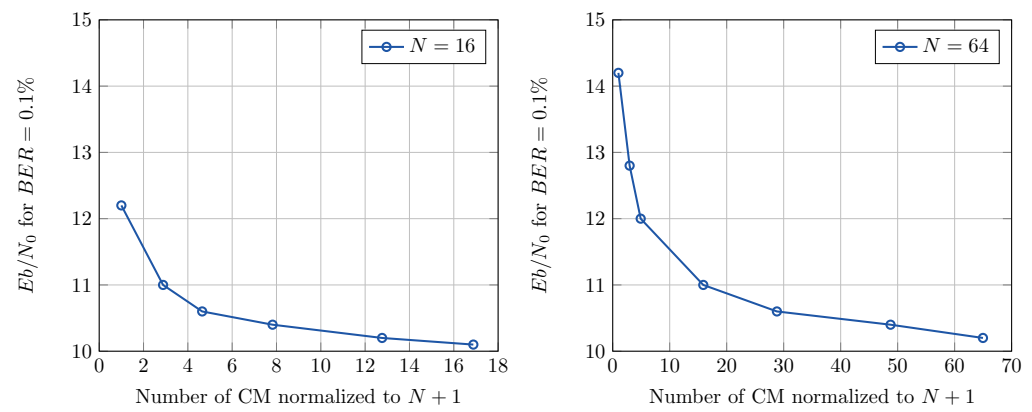

Figure 4.10: Performance and complexity trade-off for $N=16$ and $N=64$ (CM=Complex Multiplication).

of the proposed demodulator with $P=N-1$. To clarify the complexity and performance trade-off, the $E_{b} / N_{0}$ required for $B E R=0.1 \%$ versus complexity is illustrated in Figure 4.10 for $F T=16$ and $F T=64$. The complexity is calculated in terms of the number of complex multiplications and is normalized to the complexity of the conventional demodulator (equivalent to $P=0$ ). In both cases for a complexity around $50 \%$ of the complexity of $P=N-1$ the $E_{b} / N_{0}$ required for $B E R=0.1 \%$ is only around $0.5 \mathrm{~dB}$ higher than its value for $F T=1$ which is $10 \mathrm{~dB}$ (see Figure 4.8 and Figure 4.9). 


\subsection{Conclusion}

The BER performance of the autocorrelation demodulator (ACD) for DDPSK in the presence of a large frequency offset was considered. The bandwidth of the filter before the demodulator should be selected according to the target range for the frequency offset tolerance. Although DDPSK is able to tolerate frequency offset, the wide filter increases the noise bandwidth at the input of the demodulator which leads to BER performance degradation. The proposed demodulator adds additional paths to the conventional DDPSK demodulator and the sum of demodulated signals in these paths is sent to the detector. As shown in this chapter, the proposed method increases $S N R$ at the input of the detector and compensates for SNR loss resulting from the wider filter. Therefore, the BER performance remains unchanged for all values of the filter bandwidth. The simulation results revealed that the proposed method leads to more than $2 \mathrm{~dB}$ improvement in the BER performance in case the bandwidth of the filter is 8 times the symbol rate $(F T=16)$. For a wider filter i.e. a wider range of frequency offset tolerance, this gain increases. The complexity of the proposed method was also investigated and the trade-off between the BER performance and complexity was presented. 


\title{
5 \\ Low COMPLEXITY IMPLEMENTATION OF THE FSK DEMODULATOR
}

\begin{abstract}
The DFT-based demodulator for BFSK has been introduced as an offset tolerant demodulator capable of tolerating a large Carrier Frequency Offset (CFO). However, it was shown in Chapter 3 that its limitation in case of a large frequency offset is its complexity. The main source of complexity, is calculating the DFT of a window which slides over the preamble. In this chapter, the complexity is alleviated by considering the window synchronization algorithm and its implementation together. First, a new window synchronization algorithm is proposed which is designed such that an efficient class of the implementations of the Sliding DFT(SDFT), called Single Bin SDFT (SB-SDFT) in this chapter, can be used. Moreover, a stable implementation of the SB-SDFT is designed to enable zero-padding which is required by the demodulator. The complexity of the proposed algorithm implemented using the SB-SDFT, scales more efficiently compared to the conventional algorithm when the range of tolerable CFO increases. Using the proposed method, for a CFO tolerance in the order of 14.5 times the symbol rate $( \pm 1.45 \mathrm{kHz}$ for a symbol rate equal to $100 \mathrm{~Hz}$ ), the number of complex operations is reduced by more than $85 \%$ (and memory by $90 \%$ ) compared to the conventional method.
\end{abstract}

\subsection{INTRODUCTION}

A DFT-based demodulator for BFSK was introduced in Chapter 3. As discussed earlier, the prominent drawback is that a complex synchronization scheme is used to properly select the samples of a symbol for which the DFT is calculated. The computational complexity of the synchronization stems from the DFT calculation for a window which is sliding over the synchronization preamble. Various methods have been proposed for efficient implementation of the DFT

This chapter is based on [S:2] and [S:6]. 
with a sliding window $[20,24,26,29-31,42,48,53,72,73,78-80,85,100,101]$. Investigating these techniques, which are explicated in section 5.3 , reveals that the most efficient design can be obtained if the synchronization algorithm only requires a subset of the DFT bins.

In light of these facts, a low complexity synchronization algorithm is proposed in this chapter and a modified stable Single-Bin Sliding DFT (SB-SDFT) calculator is introduced for its implementation. Furthermore, the complexity of the proposed synchronization algorithm implemented using the SB-SDFT structure is analyzed and compared to an efficient implementation of the conventional algorithm.

First a brief review of the DFT-based demodulator and the conventional synchronization algorithm from Chapter 3 are explained in the next section. Section $5 \cdot 3$ looks into related work on the efficient implementation of a sliding DFT while jointly motivating the design of the proposed synchronization algorithm and the proposed SB-SDFT. Subsequently, the proposed synchronization algorithm is presented in Section 5.4. Next, section 5.5 elaborates on the proposed SB-SDFT and derives its stabilized version. The complexity analysis follows in Section 5.6 to obtain expressions for the number of operations and memory use. Numerical results and discussions are included in section 5.7. Finally, section 5.8 concludes this chapter.

\subsection{A Brief Review of the Problem}

\subsubsection{Demodulator and Synchronization Algorithm}

In this section, the DFT-based demodulator and the window synchronization algorithm from Chapter 3 are (briefly) explained again to ease the reading. The block diagram of the DFT based demodulator is shown in Figure 5.1 (this is the same block diagram from Chapter 3 and is repeated for easier reference). Signal samples pass through a low-pass filter prior to the demodulator. To tolerate large Carrier Frequency Offset (CFO), the low-pass filter might be much wider than the signal bandwidth. Moreover, complying with the Nyquist criterion necessitates a higher sampling frequency. Assuming an ideal brick-wall filter, the signal samples just before the demodulator are as follows.

$$
r_{m}[n]=\exp \left(j\left(2 \pi\left(f_{m}+f_{O}\right) n T_{s}+\theta\right)\right)
$$

where $r_{m}[n]$ is the $n^{t h}$ sample $(n=0, \ldots, N-1)$ of the $m^{\text {th }}$ symbol; $f_{m}$ is transmitted frequency during $m^{\text {th }}$ symbol which is either $f_{0}$ or $f_{1}$; $\theta$ is the phase offset, $f_{O}$ is the frequency offset and $T_{s}$ is the sampling period. After the filter, a proper set of samples (all belonging to the same symbol) is selected by a rectangular window, padded with zeros and sent to the DFT calculation block 


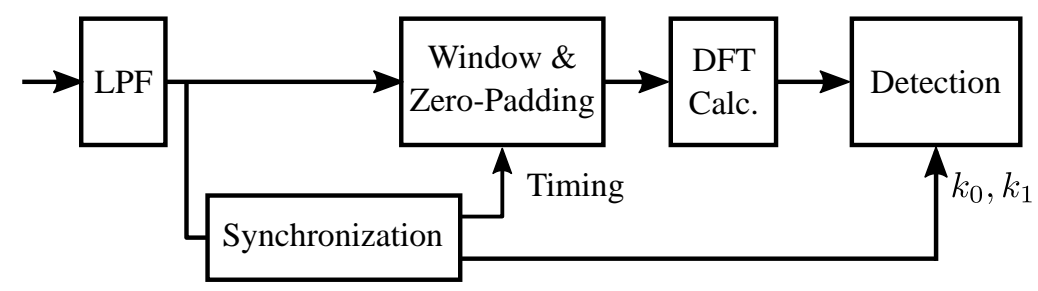

Figure 5.1: Block diagram of the DFT-based demodulator from Chapter 3

to achieve the following.

$$
X_{k, m}=\sum_{n=0}^{N I-1} \mathbf{x}_{m}^{I}[n] \exp \left(-j \frac{2 \pi k n}{N I}\right)
$$

where, $X_{k, m}$ is the $k^{t h}$ bin of the DFT calculated for $m^{t h}$ symbol. $I$ is the zero-padding factor which means that $N$ samples of the signal are padded by $N(I-1)$ zeros to achieve a zero-padded sequence $\mathbf{x}_{m}^{I}$ with length $N I$ as the input of the DFT. Finally, the decision for each symbol of BFSK is made based on the magnitudes of $X_{k, m}$. Zero-padding is used to improve the bin resolution of the DFT as explained in Chapter 3. The frequency separation of the BFSK modulation is assumed to be equal to the symbol rate $\left(f_{1}=+1 / 2 T\right.$ and $f_{0}=$ $-1 / 2 T$ in $(5.1))$.

Two phases can be distinguished in the demodulator operation. First, the window must be aligned to the received symbols; otherwise, frequency components of the adjacent symbols introduce inter-symbol interference. This window alignment is done during the synchronization phase. Furthermore, the DFT frequency bins corresponding to the frequency of symbol one $\left(k_{1}\right)$ and zero $\left(k_{0}\right)$ of the BFSK modulation are determined in this phase [89]. In the detection phase, the DFT is calculated for the samples of each symbol. For each symbol the decision is made by comparing the magnitude of the DFT bins $k_{1}$ and $k_{0}$.

The synchronization algorithm discussed in Chapter 3 has been introduced by Hara et al. [44] and, in the rest of this chapter, is referred to as Hara synchronization or the conventional synchronization algorithm. It uses a preamble of alternating ones and zeros. The number of symbols in the preamble is denoted by $L$. For each symbol, windows with different delay values, between 0 and $N-1$, are considered. Only one of these windows is fully aligned with one symbol and others include samples of two consecutive symbols. The DFT is calculated for each window. Figure 5.2 illustrates three symbols of the preamble and the windows in case of four samples per symbol (this is the same block diagram from Chapter 3 and is repeated for easier reference). It also shows how the spectrum varies for different delays. The symbols of preamble are called Even and Odd depending on their index $m=1, \ldots L$. All DFT magnitudes cor- 
responding to Odd symbols (Even symbols) in the preamble are added together to achieve the following.

$$
\begin{gathered}
S_{i}^{O}(k)=\sum_{n=1}^{L / 2} X_{k, i}^{2 n-1} \\
S_{i}^{E}(k)=\sum_{n=1}^{L / 2} X_{k, i}^{2 n}
\end{gathered}
$$

where $i=0, \ldots, N-1$ is the window delay. This is visualized in Figure 5.2. The double-headed arrows denote windows. Each row of arrows correspond to a certain delay value as shown in the figure. Solid (dotted) arrows show the windows for an Odd (Even) symbol with different delay values. In fact, (5.3) and (5.4) calculate the sum of the DFT magnitudes for solid windows and the sum of those for dotted windows, respectively.

To synchronize the window, the Hara algorithm finds the delay value for which $R_{i}$ in the following equation is maximized.

$$
R_{i}=\left[S_{i}^{E}\left(k_{i}^{E}\right)-S_{i}^{E}\left(k_{i}^{O}\right)\right]+\left[S_{i}^{O}\left(k_{i}^{O}\right)-S_{i}^{O}\left(k_{i}^{E}\right)\right],
$$

where $k_{i}^{E}$ and $k_{i}^{O}$ are the bins with maximum magnitude in $S_{i}^{E}$ and $S_{i}^{O}$, respectively. Finding the maximum of (3.9) is simply finding the delay value which maximizes the difference shown by $D$ in Figure 5.2. When the delay value is found, the $k_{1}$ and $k_{0}$ can be achieved from $k_{i}^{O}$ and $k_{i}^{E}$, respectively.

\subsubsection{A Quick LoOK Aт Complexity}

The number of complex multiplications (CM) and complex additions (CA) required for the synchronization and detection phases are listed in Table 5.1 for a system with $N=I=8$ and $L=16$ (the same as Chapter 3). The detailed calculations for complexity can be found in Chapter 3. Synchronization is done once in a packet so its complexity must be divided by the number of symbols in a packet to achieve the complexity per symbol. According to Table 5.1, the packet length for which detection complexity per symbol exceeds synchronization complexity is more than 1400 symbols. This is much larger than the packet length in UNB communications (in order of 200 symbols). Moreover, the operations required for synchronization are done in a shorter period of time compared to the detection phase which leads to high instantaneous power consumption as a consequence of higher clock frequencies. This makes it unsuitable for applications where maximum instantaneous power is limited. Thus, the complexity of the synchronization algorithm is always dominant and needs to be alleviated.

As mentioned before in the Hara synchronization algorithm, the DFT is calculated for a window which slides over the preamble. This plays the paramount role in the complexity of the demodulator but can be implemented efficiently 


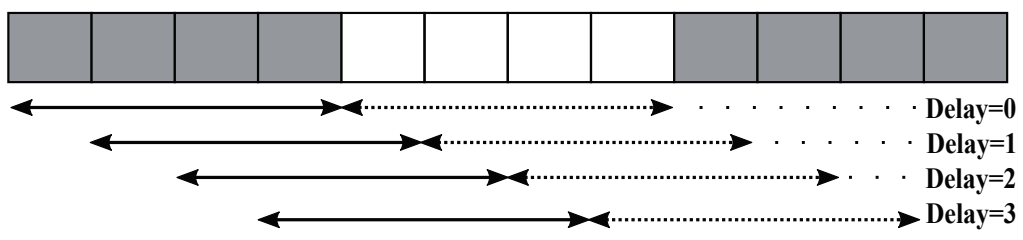

Odd Symbols ( $\longrightarrow$ ) $\square$ Even Symbols ( ......)
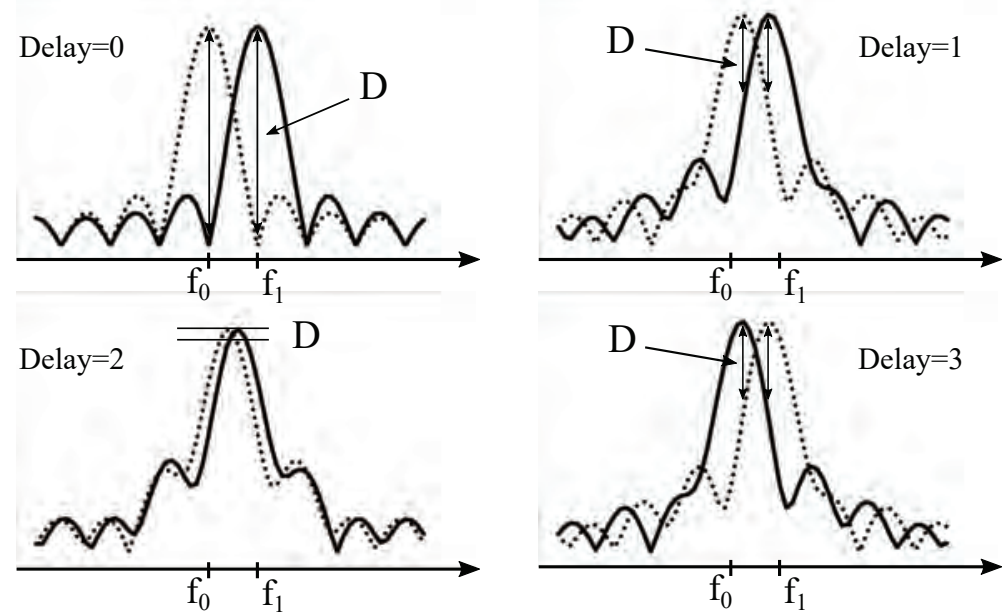

Figure 5.2: The first three symbols of a preamble, the windows of different delay values for the first two symbols and an example of spectrum variation when there are 4 samples per symbol. Solid and dotted lines show windows and spectra of odd and even symbols, respectively.

Table 5.1: Complexity of different phases in the demodulator for $N=I=8$ and $L=16$. CM and CA stand for complex multiplication and complex addition, respectively.

\begin{tabular}{c|c|c}
\hline Demodulator Phase & CA & CM \\
\hline Detection (/Symbol) & 14 & 16 \\
\hline Synchronization (/Packet) & 46080 & 23040 \\
\hline
\end{tabular}

using Sliding DFT algorithms. The next section elaborates on the efficient implementation of the Sliding DFT reviewing related literature. 


\subsection{Related Work on the Sliding DFT (SDFT)}

Calculating the DFT for a window which is sliding over a sequence is called Sliding DFT (SDFT). As a computationally intensive block, many researchers have investigated the efficient implementation of the SDFT. In case of a sliding window, only one sample (or a few samples) is (are) different between the current set of input samples of the DFT and the previous set. Exploiting this property, various methods have been proposed $[20,24,26,29-31,42,48,53,72,73,78-$ $80,85,100,101]$. By reusing calculations done for each window, the DFT of the next window can be calculated in a more efficient way. For a better understanding, we categorize the methods into two general groups. The first group, Complete SDFT (C-SDFT), includes algorithms that calculate all the DFT bins for each window in a single structure; whereas, the second category, SingleBin SDFT (SB-SDFT), covers algorithms which derive only a single bin of the DFT. These categories are explained in the next two subsections. Although the final design in this work belongs to the SB-SDFT category, review of the C-SDFT methods is necessary. It helps us to derive an efficient implementation of the Hara synchronization algorithm (the algorithm explained in the previous section) which is used as a benchmark for complexity comparison. In the last subsection, the conclusions that can be drawn from literature are pointed out. This subsection also describes how this insight provokes the design of the proposed algorithm and the proposed SB-SDFT.

\subsubsection{Complete SDFT (C-SDFT)}

One of the initial examples of an SDFT has been introduced in $[20,30,31]$. Based on the conventional Decimation-in-Time FFT structure and storing calculated intermediate values, this method decreases the complexity of the FFT calculation for the new coming sample from $\mathscr{O}\left(N \log _{2} N\right)$ in the FFT to $\mathscr{O}(N)$ [30]. However, it increases the required memory as it needs to store all the intermediate values. In our complexity analysis in section 5.6 , this structure is considered for the implementation of the Hara synchronization algorithm; therefore, it is worth to explain it in more detail. Figure 5.3 shows the FFT structure used in [30] where the old window samples are shown in a dashed box (in grey) and the white circles show butterflies of the FFT. Samples $x(n-16)$ to $x(n-1)$ (inside the dashed box) belong to the $(n-1)^{t h}$ window. When sample $x(n)$ is added, samples $x(n-15)$ to $x(n)$ are included in the $n^{\text {th }}$ window and the FFT for them must be calculated. It can be seen that all required butterflies except for the highlighted ones can be derived from butterfly outputs of the $(n-1)^{t h}$ window. In other words, when the window slides one sample, only one butterfly from each group of butterflies using the same twiddle factor (those in one dashed oval) needs to be calculated. For an $N$-point FFT, only $N-1$ butterflies must be calculated when the window slides for one sample. This method is one of the most efficient ways to calculate the C-SDFT and is called Sliding FFT (SFFT) hereafter. 


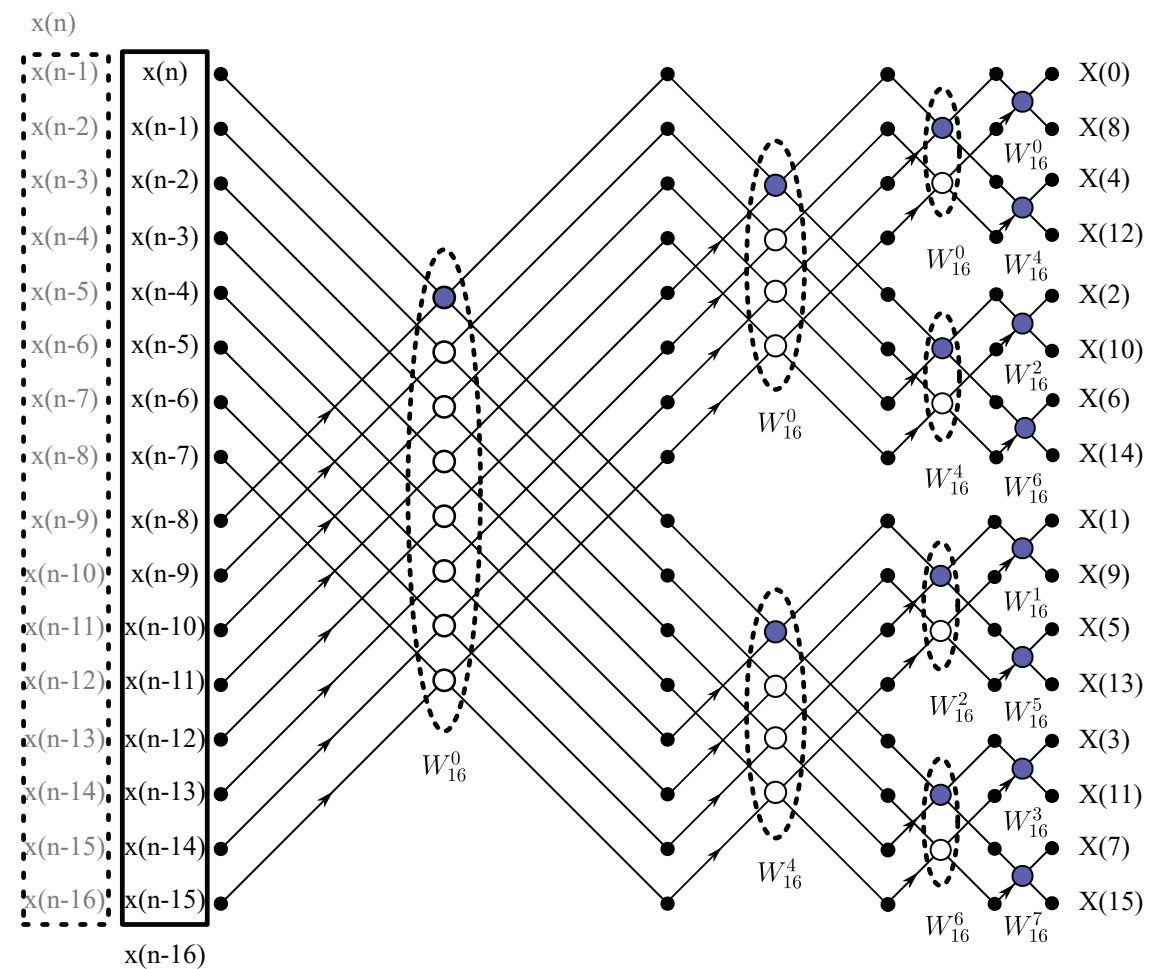

Figure 5.3: The Sliding FFT structure introduced by [30]. The dashed box in the left includes the samples belonging to the old window. When the window slides one sample, the samples in the solid box will be new inputs to the FFT. The butterflies enclose in each dashed oval are called a group of butterfly. Each group uses the same twiddle factor for calculations.

Interpreting the FFT structure as a prism, Wang et al. [100] proposed a method to calculate the SDFT. Although its complexity is more than that of the SFFT in [30], it can be implemented in parallel for faster calculation [101] which is not necessary in our target application as it involves very low data rates. Rewriting the sliding DFT definition and using time shift properties, Montoya et al. have proposed a sliding DFT method with almost 50\% reduction in the number of complex multiplications compared to the SFFT [73]. This idea is also extended to a Radix-4 decomposition in [72] which achieves even more savings at the cost of limiting the DFT points to a power of four. Despite the complexity reduction achieved in $[72,73]$, these cannot be used when zero-padding is involved which is the case in our target application. A guaranteed stable recursive algorithm for calculation of all bins is proposed in [78] which is based on a single bin recursive structure from the same author in [80]. This method is only applicable in a hopping scenario where each window hops $N / 4$ samples ( $N$ is the DFT size). 


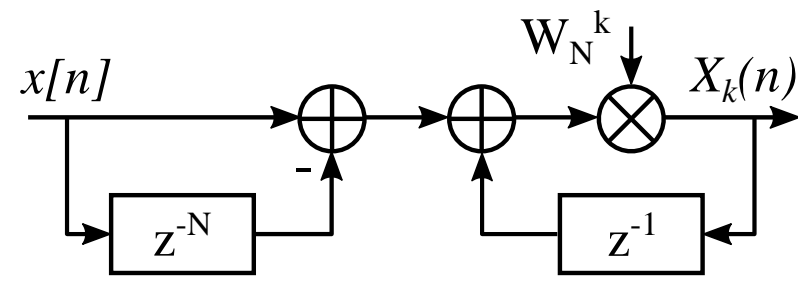

Figure 5.4: The simplest form of the SB-SDFT structure [48]

Another recursive algorithm for the C-SDFT is introduced in [53] utilizing the observer theory in the control systems. The algorithm calculates the C-SDFT while solving the stability problem associated with recursive SDFTs with less memory than what is required by the SFFT. However, it cannot work when zero-padding is used in the input sequence. Among the C-SDFT methods, the technique in [30] (the first mentioned method in this section) is the best one for implementing the Hara synchronization algorithm due to its simplicity and possibility of using zero-padding.

\subsubsection{Single Bin SDFT (SB-SDFT)}

The second group of SDFT algorithms are those which focus on calculating a single bin of the DFT (SB-SDFT). The core idea of such systems is based on the shifting property of the Fourier transform [48]. To clarify, consider the $N$-point DFT for $\mathbb{X}_{n}=\{x(n-N+1), \ldots, x(n)\}$ as follows

$$
X_{k}(n)=\sum_{i=0}^{N-1} x(n-N+i+1) W_{N}^{-k i},
$$

where $X_{k}(n)$ is the $k^{t h}$ DFT bin of $\mathbb{X}_{n}$ and $W_{N}=\exp (j 2 \pi / N) . X_{k}(n)$ can be written in terms of $X_{k}(n-1)$, which is the DFT for $\mathbb{X}_{n-1}=\{x(n-N), \ldots, x(n-$ $1)\}$, as follows.

$$
X_{k}(n)=X_{k}(n-1) W_{N}^{k}-x(n-N) W_{N}^{k}+x(n) W_{N}^{-(N-1) k}
$$

Noticing that $W_{N}^{N k}=1,(5 \cdot 7)$ can be rewritten as:

$$
X_{k}(n)=W_{N}^{k}\left(X_{k}(n-1)-x(n-N)+x(n)\right)
$$

The block diagram of a filter which realizes (5.8) is depicted in Figure 5.4. It calculates the $k^{t h}$ bin of an $N$-point DFT by implementing the DFT series for each bin as an IIR filter.

With each incoming sample (each filter iteration), the value of the $k^{t h}$ bin of an $N$-point DFT is updated for the last $N$ samples. As a result, when the window 
hops one sample, only one complex multiplication and two complex additions are required to obtain the $k^{\text {th }}$ bin for the new set of samples.

As can be seen, there is a feedback loop with a pole on the unity circle of the complex plane. This pole makes the system conditionally stable. In real applications, the limited precision of the twiddle factors $\left(W_{N}^{k}\right)$ can push the pole out of the unit circle and cause instability. In [48] a damping factor, $r$, is used to force the pole inside the unit circle while compromising the precision of the DFT calculation and causing errors. Nonetheless, if the damping factor is chosen close to one, the error can be kept small enough for many applications. Due to using the damping factor $r$, the method in [48] is also called rSDFT. To overcome the stability problem without compromising precision, a modulated SB-SDFT algorithm (modulated-SDFT) has been introduced in [26]. In this method the time-domain modulation duality of the Fourier Transform is used to shift any desired frequency component to the zero frequency. In this way, the pole in the feedback loop is equal to one and the filter will always be stable. Although both stability and precision are addressed in this method, the complexity increases considerably since the input samples need to be modulated with a proper twiddle factor.

\subsubsection{Concluding Literature}

Considering previous work on the sliding DFT, one may conclude that C-SDFT methods are more efficient if all bins of the DFT are required; however, if a subset of the bins is needed, the complexity of an SB-SDFT is lower [79] (less than half of all bins when the SB-SDFT is compared to the SFFT). The Hara synchronization algorithm needs all bins of an NI-point DFT. Now, if a new algorithm is designed which only uses a subset of the bins belonging to an NI-point DFT, the complexity can be decreased using an SB-SDFT implementation. This is the incentive for designing the proposed window synchronization algorithm.

On the other hand, as mentioned in Chapter 3, zero-padding is necessary for synchronization and detection. None of the mentioned SB-SDFT algorithms can be used together with zero-padding ${ }^{1}$. Hence, for efficient implementation of the proposed algorithm a modified SB-SDFT is needed that incorporates zeropadding. In the next section, a synchronization algorithm is proposed which only needs a subset of the NI-point DFT bins. In section 5.5, a stable SB-SDFT structure is derived for efficient implementation of the proposed algorithm.

\footnotetext{
${ }^{1}$ When working on the idea of the low complexity synchronization algorithm and its implementation in [S:6], there was no previous paper on the Sliding DFT with zero-padding. In the process of final thesis preparation, we noticed that a work on the SB-SDFT with zero-padding [23] was independently presented in another conference almost at the same time our work [S:6] was presented.
} 


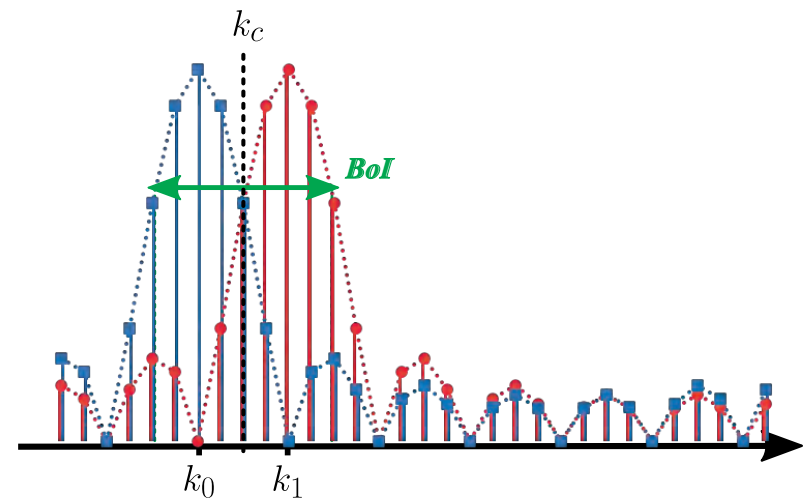

Figure 5.5: The spectrum of the signal and the BoI

\subsection{The Proposed Window Synchronization Algorithm}

So far, it has been concluded that using only a subset of the bins of an NI-point DFT for window synchronization reduces complexity compared to the Hara synchronization method. Before introducing the proposed synchronization algorithm, it is needed to check whether it is feasible to only rely on a subset of bins for synchronization (without losing signal information). This is done in the first subsection where a set called Bins of Interest (BoI) is defined and the general concept of the proposed synchronization algorithm is introduced. Afterwards, the algorithm is described in detail.

\subsubsection{Bins of Interest and the Proposed Synchronization Concept}

In the presence of a large frequency offset (multiple times the symbol rate) a filter wider than (multiple times) the signal bandwidth before the demodulator is needed to capture the signal. It means that the sampling frequency should be higher than the actual information bearing bandwidth of the signal. On the other hand, using zero-padding increases the number of bins that must be calculated including those which are out of the signal bandwidth. As a result of this oversampling and zero-padding, only a small part of the spectrum calculated by an NI-point DFT includes signal information. These bins of the spectrum are called Bins of Interest hereafter and BoI for each DFT is defined as the set which includes these bins. Since the signal information resides in the BoI, solely the BoI can already provide a correct synchronization without loss of information. The BoI is in the vicinity of the frequency offset in the baseband signal which is called center frequency $f_{c}$ in the following $\left(f_{c}=f_{O}\right)$. This is shown in Figure 5.5 for a case where the sampling frequency is 8 times the symbol rate and a zero-padding factor of 4 is used (32-point DFT). In Figure 5.5, the $k_{0}, k_{1}$ and $k_{c}$ correspond to the frequencies $f_{0}, f_{1}$ and $f_{c}$, respectively. 


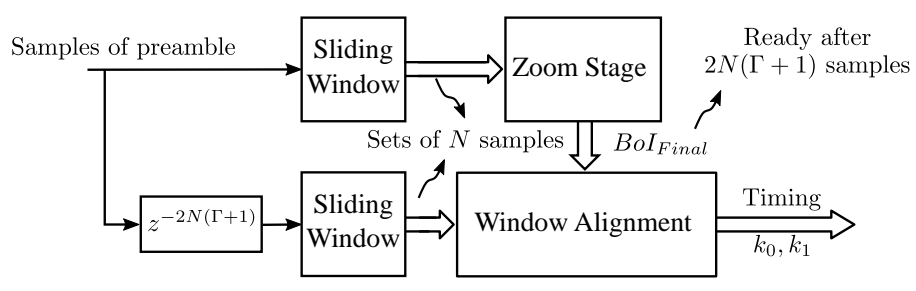

Figure 5.6: The block diagram of the proposed synchronization concept

Since the frequency offset is assumed to be unknown, there is no prior knowledge about the whereabouts of the BoI in the spectrum. The proposed method aims first at finding the BoI to limit the number of DFT bins required for the synchronization later on. Using a subset of the DFT bins, not only SB-SDFT algorithms can be used to decrease complexity but also fewer bins need to be stored during synchronization which considerably decreases memory requirements.

In the proposed synchronization concept an extra process is added to the synchronization, splitting it into two stages; the zoom stage and the window alignment stage. The concept of the proposed synchronization method is shown in Figure 5.6. Input samples pass through a sliding rectangular window providing sequences of length $N$. First, in the zoom stage (explained in the next subsection), the BoI of an NI-point DFT called BoI $I_{\text {Final }}$ is detected. Secondly, BoI $I_{\text {Final }}$ is sent to the window alignment stage where a proper window delay is obtained only calculating the DFT bins in $B o I_{\text {Final }}$ and using the algorithm illustrated in Figure 5.2. To compensate for the delay introduced by the zoom stage, an equivalent delay $\left(z^{-2 N(\Gamma+1)}\right)$ has been inserted before the Window Alignment block. It enables the algorithm to reuse the same samples used in the zoom stage for the window alignment. It avoids an increased number of preamble symbols for the proposed synchronization. In the next subsection, the zoom stage is explained in detail.

\subsubsection{The Zoom Stage}

The target of this stage is to find the BoI of an NI-point DFT $\left(B o I_{\text {Final }}\right)$ which covers the DFT bins corresponding to the BFSK modulation frequencies $k_{1}$ and $k_{0}$. For this purpose, a step-by-step zooming approach is utilized which searches for the center bin in an NI-point DFT, $k_{c, F i n a l}$. The center bin is the bin closest to the center frequency $\left(f_{c}\right)$ of the signal. The $B o I_{\text {Final }}$ is calculated using the $k_{c, \text { Final }}$. Before elaborating on the step-by-step zooming algorithm, parameters are defined. $T$ is the symbol period and $N$ is the number of samples per symbol. $I=2^{\Gamma}$ is the zero-padding factor. As mentioned earlier, the zero-padding factor for the DFT-based demodulator is selected to be $I=8$, yet, for the proposed algorithm it can be any power of two, $2^{\Gamma}$. Moreover, the frequency separation of the BFSK modulation is equal to the symbol rate $\left(f_{1}=f_{c}+1 / 2 T\right.$ and $f_{0}=$ $f_{c}-1 / 2 T, f_{c}=f_{O}$ in baseband). 


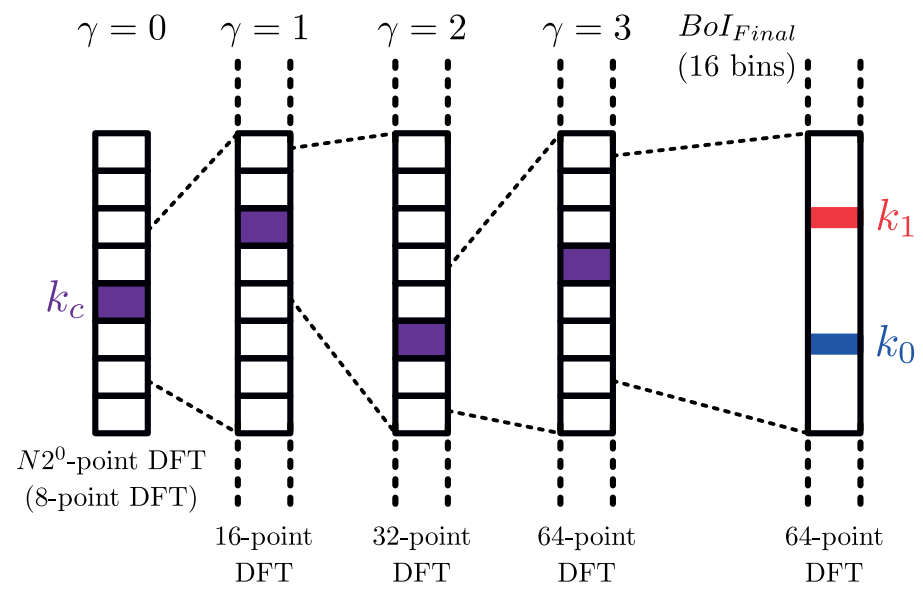

Figure 5.7: A visual description of step-by-step zooming for $N=8$ and $I=8$ (or $\Gamma=3)$.

The zoom stage includes $\Gamma+1$ steps while the zero-padding factor at step $\gamma$ is equal to $2^{\gamma}(\gamma=0, \ldots, \Gamma)$. A visual illustration of the step-by-step zooming is shown in Figure 5.7. In this figure $N=8$ and $I=8$ which is equivalent to $\Gamma=3$. At each step $\gamma$, the bin $k_{c}^{\gamma}$ of an $N 2^{\gamma}$-point DFT, which is closest to $f_{c}$, is detected based on calculating only the bins within $B o I_{\gamma}$. Then, an estimate of the next step center bin, $\hat{k}_{c}^{\gamma+1}$, is calculated using $k_{c}^{\gamma}$. Following on that, $\hat{k}_{c}^{\gamma+1}$ is exploited to determine the BoI for step $\gamma+1, B o I_{\gamma+1}$. Subsequently, in step $\gamma+1$, the actual center bin $k_{c}^{\gamma+1}$ is detected by calculating only a subset of the bins of an $N 2^{\gamma+1}$-point DFT in $B o I_{\gamma+1}$. Continuing this procedure, the center frequency of an $N I$-point DFT is obtained which is used to find $B o I_{F i n a l}$.

The zoom stage starts with the first step, $\gamma=0$, which uses an $N$-point DFT i.e. with a zero-padding factor of one (or no zeros). The BoI for the first step $(\gamma=0)$ includes all the bins of the $N$-point DFT. All points of the $N$-point DFT are required in the very first step because the center frequency can be anywhere in the spectrum that can be covered by the sampling frequency. Thus, we need to look at all bins at the first step and then we can limit ourselves to the $B o I_{\gamma}$ calculated for further steps. Methods for calculating $k_{c}^{\gamma}$, calculating $\hat{k}_{c}^{\gamma+1}$ from $k_{c}^{\gamma}$ and determining $B o I$ are explicated as follows.

\section{Calculating $k_{c}^{\gamma}$}

At step $\gamma$, a window of length $N$ slides for $2 N$ hops (in shifts of one sample) over the preamble (this is equal to the number of samples for two symbols) and the DFT is calculated for each window. For each one-sample shift of the window, only the bins of an $\mathrm{N} 2^{\gamma}$-point DFT inside $\mathrm{BoI}_{\gamma}$ are calculated. So there will be 
$2 N$ DFTs for which the bin with maximum magnitude is changing between $f_{1}$ and $f_{0}$. An example of the windows for $N=4$ and how the spectrum changes can be seen in Figure 5.2.

The magnitudes of all these DFTs for each bin are added. Then, the bin for which this sum is maximum is closest to the center frequency $k_{c}^{\gamma}$. Thus, $k_{c}^{\gamma}$ is obtained as follows.

$$
k_{c}^{\gamma}=\underset{k \in B O I_{\gamma}}{\arg \max } \mathscr{X}_{k}^{\gamma},
$$

where:

$$
\mathscr{X}_{k}^{\gamma}=\sum_{i=0}^{2 N-1}\left|X_{k, i}^{\gamma}\right|^{2}
$$

$X_{k, i}^{\gamma}$ is the DFT for the $k^{t h}$ bin and window $i=0, \ldots, 2 N-1$ in step $\gamma$. The first window can start from any part of either a "one symbol" or a "zero symbol" in the preamble. Proving why the bin achieved using this method is actually the center bin is dealt with in Appendix A.

\section{Calculating $\hat{k}_{c}^{\gamma+1}$ from $k_{c}^{\gamma}$}

The zero-padding factor is doubled between two consecutive steps. It means that zeros are added so that the length of the sequence over which DFT is calculated (including zeros) becomes twice of its value in the previous stage. This actually adds a new "interpolated" bin between each two bins of an $N 2^{\gamma}$-point DFT to achieve an $N 2^{\gamma+1}$-point DFT. Therefore, the center bin in step $\gamma+1$ can be estimated as $\hat{k}_{c}^{\gamma+1}=2 k_{c}^{\gamma}$. Notice that $f_{c}$ might not be matched to a bin due to the arbitrary CFO. In this case two adjacent bins close to the $f_{c}$ have the largest magnitudes among all (the magnitudes are exactly the same if $f_{c}$ is exactly in the middle of the two bins and there is no noise). In such cases a noisy received signal and leakage causes the calculated $\hat{k}_{c}^{\gamma+1}$ to deviate from the actual center bin in step $\gamma+1$. That is why the center frequency should be detected step-by-step so that the final center frequency bin in the $N I$-point DFT is selected correctly. $\hat{k}_{c}^{\gamma+1}$ is used to determine $B o I_{\gamma+1}$ as follows.

\section{Determining BoI $I_{\gamma+1}$ and BoI Final}

To account for any erroneous detection due to the spectral leakage and noise, $I$ bins (equal to the zero-padding factor) in the vicinity of the estimated center bin are considered as follows.

$$
B o I_{\gamma+1}=\{k \mid k \in[\min (a, b), \max (a, b)]\},
$$

where:

$$
\begin{array}{rr}
a=\left(\hat{k}_{c}^{\gamma+1}-I / 2\right) & \bmod \left(N 2^{\gamma+1}\right) \\
b=\left(\hat{k}_{c}^{\gamma+1}+I / 2-1\right) & \bmod \left(N 2^{\gamma+1}\right)
\end{array}
$$


In (5.12) and (5.13), mod is modulo operator.

In the final step, when $k_{c}^{\text {Final }}$ is calculated, the final BoI used for the window alignment stage $\left(B o I_{\text {Final }}\right)$ is determined. $B o I_{\text {Final }}$ should include the frequency bins corresponding to symbols 1 and 0 of the BFSK modulated signal $\left(k_{1}\right.$ and $k_{0}$, respectively). Since the frequency separation of the BFSK modulation is equal to the symbol rate $\left(R_{\text {Sym }}\right)$, when the zero-padding factor is $I$ and the sampling frequency is $N R_{\text {Sym }}$, there are $I-1$ bins between $k_{1}$ and $k_{0}$. To reduce the effect of noise, more than $I$ bins are considered. If the number of bins in the $B o I_{\text {Final }}$ (its cardinality) is denoted by $\left|B o I_{\text {Final }}\right|$, the $B o I_{\text {Final }}$ is determined as follows.

$$
B o I_{\text {Final }}=\{k \mid k \in[\min (d, e), \max (d, e)]\},
$$

where:

$$
\begin{array}{rr}
d=\left(k_{c, \text { Final }}-\mid \text { BoI }_{\text {Final }} \mid / 2\right) & \bmod (N I) \\
e=\left(k_{c, \text { Final }}+\left|B_{0} I_{\text {Final }}\right| / 2-1\right) & \bmod (N I)
\end{array}
$$

The size of BoI Final is optimized to achieve the best BER performance using simulations which are presented in section 5.7.

A detailed block diagram of the proposed synchronization algorithm is also shown in Figure 5.8 (top part is the zoom stage and the bottom is the window alignment). Both the zoom stage and the window alignment stage receive a set of $N$ samples from a sliding window as shown in Figure 5.6. The sets are the result of a rectangular window which slides over the preamble by one-sample shifts. The zoom stage is composed of three blocks. The first block calculates the DFT, the second calculates $k_{c}^{\gamma}$ and the third calculates $\hat{k}_{c}^{\gamma+1}$ and $B o I_{\gamma+1}$. The BoI Final is the output of the zoom stage which is sent to the window alignment stage. The window alignment stage is similar to the algorithm explained in section 5.2 (see Chapter 3 for more detail). Getting samples of windows with different delays over the preamble, the DFT calculation block calculates the DFT magnitudes for bins in BoI Final . These values are passed to the next block which calculates $R_{i}$ similar to (5.5)(where $0 \leq i \leq N-1$ are the window delays) but only for the bins in $B o I_{\text {Final }}$. Finally, the third block finds the $i$ value for which $R_{i}$ is maximum and finds $k_{0}$ and $k_{1}$ in the $B o I_{\text {final }}$. This will be the proper window delay (the timing information) and, together with $k_{0}$ and $k_{1}$, it is used during the detection phase. For the DFT calculation blocks in the zoom and the window alignment stages, an SB-SDFT calculator can be used which is introduced in the next section.

\subsection{The Proposed SB-SDFt with Zero-Padding}

In the previous section, a synchronization algorithm was presented. The second issue mentioned in the conclusion of the literature review for efficient implementation of the SDFT is an SB-SDFT scheme in the presence of zero-padding. 

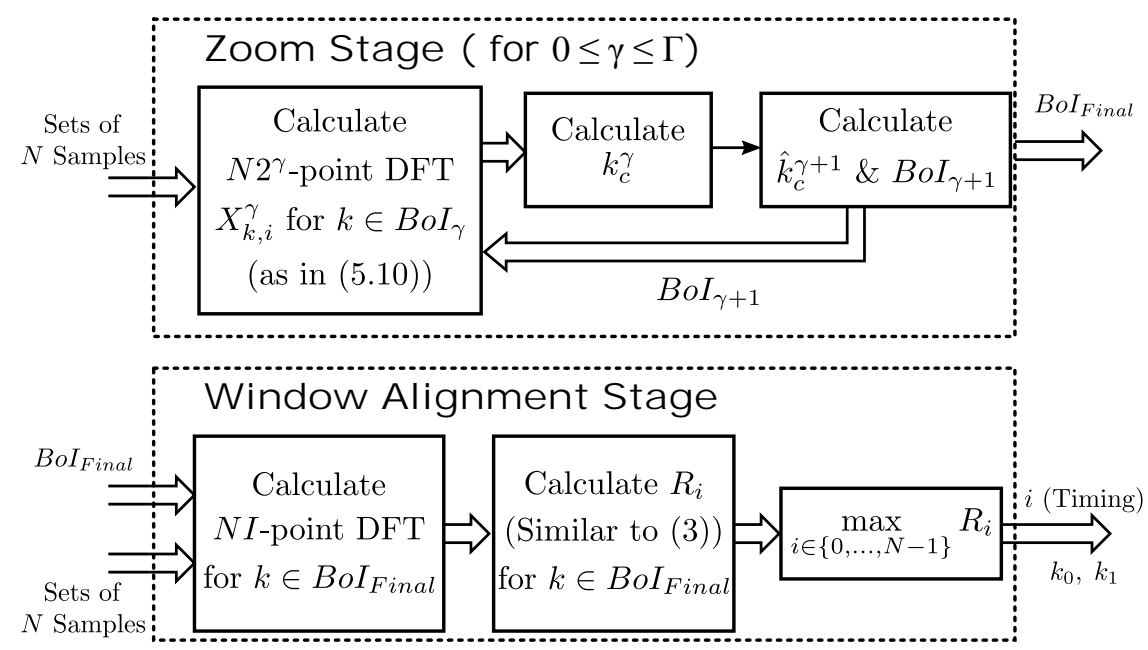

Figure 5.8: The block diagram of the proposed synchronization algorithm; BoI stands for Bins of Interest

Aforementioned implementations for an SB-SDFT (see section 5.3) do not take the zero-padding into account. The main derivation of these algorithms is based on the periodicity of the twiddle factors. When each set of the samples is padded with zeros before the DFT calculation, this periodicity is violated due to zeros appended to the samples sequence. This leads to incorrect calculation by SBSDFT schemes. For the proposed synchronization algorithm, a new SB-SDFT algorithm is needed to incorporate zero-padding. A procedure similar to the conventional SB-SDFT derivation in $[26,48]$ and $[78]$ is followed.

The $k^{t h}$ bin of an $M$-point DFT over a set of $N$ samples, $\mathbb{X}_{n}=\{x(n-N+$ $1), \ldots, x(n)\}$, which are padded with $M-N$ zeros, is as follows.

$$
X_{k}(n)=\sum_{i=0}^{N-1} x(n-N+i+1) W_{M}^{-k i}
$$

where $W_{M}=e^{j \frac{2 \pi}{M}}$ and the last $M-N$ terms of the sum are ignored as they are zero. The $k^{t h}$ DFT bin in (5.17) can be obtained using the $k^{t h}$ DFT bin for sample set $\mathbb{X}_{n-1}=\{x(n-N), \ldots, x(n-1)\}$ as follows.

$$
X_{k}(n)=X_{k}(n-1) W_{M}^{k}-x(n-N) W_{M}^{k}+x(n) W_{M}^{-(N-1) k}
$$

where $X_{k}(n-1)$ is the $k^{t h}$ DFT bin for $\mathbb{X}_{n-1}$. When there is no zero-padding, $N=M$ and $W_{M}^{-(N-1) k}$ in the last term of (5.18) can be simplified to $W_{M}^{k}$ leading to the known SB-SDFT equation (see (5.8)). If zero-padding is used, this simplification is not valid anymore. This is the violation of the periodicity mentioned above and necessitates a modified version of the SB-SDFT. In case of zero-padding, $N \neq M$ and (5.18) can be written as follows. 


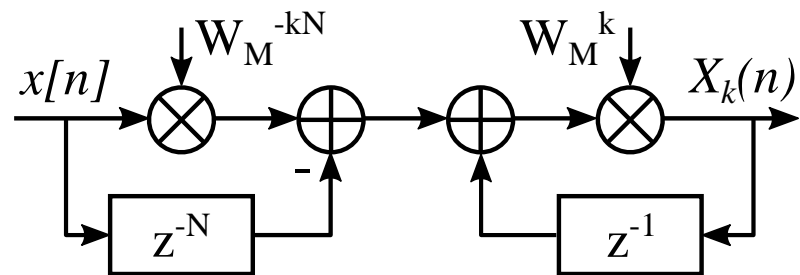

Figure 5.9: The modified SB-SDFT filter

$$
X_{k}(n)=W_{M}^{k}\left(X_{k}(n-1)-x(n-N)+x(n) W_{M}^{-N k}\right)
$$

Equation (5.19) can be seen as a filter taking samples of $x$ and generating the $k^{t h}$ DFT bin while sliding the window by one sample. The transfer function of the filter is:

$$
H(z)=\frac{W_{M}^{-k N}-z^{-N}}{W_{M}^{-k}-z^{-1}}
$$

The block diagram of such a filter is also depicted in Figure 5.9. The SB-SDFT with zero-padding has an extra multiplication in the first loop compared to the SB-SDFT in Figure 5.4. Similar to the conventional SB-SDFT in [48], it has a pole on the unity circle. The stability of this modified version of SB-SDFT can be guaranteed using a damping factor $r$ (similar to the idea proposed in [48]). Another method is to extend this derivation to achieve a modified version of the modulated-SDFT introduced in [26]. The former compromises precision while the latter increases complexity almost twice. Using a damping factor causes an error in the calculated DFT values. In our proposed method the exact value of the DFT is not the target but the relative value of different bins is important. As a result, to avoid the complexity of the modulated-SDFT, the modified SB-SDFT method is stabilized by adding a damping factor. To change the pole from $W_{M}^{k}$ to $r W_{M}^{k}$, the $z$ in $(5.20)$ is replaced with $z / r$. This leads to the following transfer function.

$$
H(z)=\frac{W_{M}^{-k N}-r^{N} Z^{-N}}{W_{M}^{-k}-r z^{-1}}
$$

The block diagram of the stable filter is shown in Figure 5.10. For each loop, one multiplication between a complex number and a real number is added which is composed of two real multiplications. The extra multiplications in the second loop can be integrated into the twiddle factor. To do so, the nominator and the denominator of the transfer function are multiplied with $r^{-1}$ to achieve: 


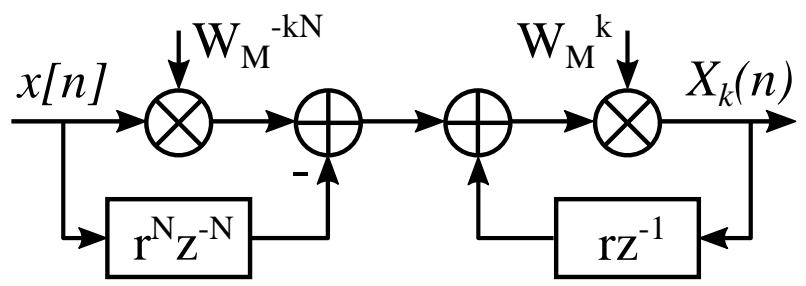

Figure 5.10: The modified SB-SDFT filter stabilized using $r$

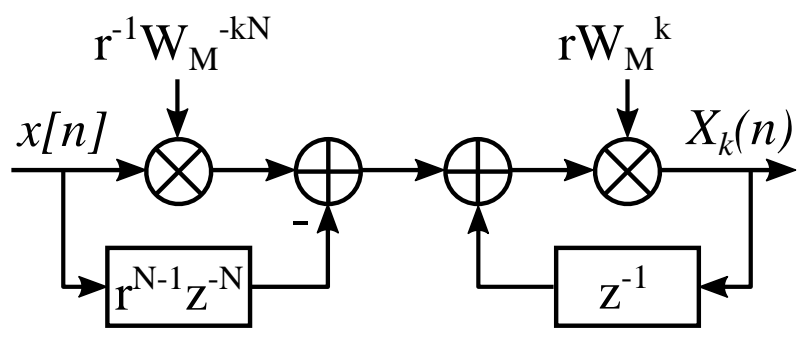

Figure 5.11: Modified and stabilized SB-SDFT filter with reduced multiplication

$$
H(z)=\frac{r^{-1} W_{M}^{-k N}-r^{N-1} z^{-N}}{r^{-1} W_{M}^{-k}-z^{-1}}
$$

The final block diagram is shown in Figure 5.11. Interestingly, the one extra multiplication compared to (5.20) which is in the first loop is independent of the bin number and can be shared between filters which are calculating different DFT bins. The effect of the damping factor on the demodulator performance and the proper value for it are investigated using simulations presented in section $5 \cdot 7$.

\subsection{Complexity Analysis}

In the first subsection, the number of complex multiplications and complex additions (shown by CM and CA, respectively) required for the DFT calculation are obtained. Next, the memory usage is calculated including the memory needed to compute the Sliding-DFT and the memory required to store $S_{i}^{O}$ and $S_{i}^{E}$ (in (5.3) and (5.4), respectively) and the twiddle factors. In the final design, the zeropadding factor $I$ is 8 ; however, in the following, parameter $I$ is used for clarity. The proposed SB-SDFT is shown again for easier reference in Figure 5.12. The dotted circles in Figure 5.12 demonstrate different operations and/or memory required for the calculation of the SB-SDFT. Notice that the calculations shown in Figure 5.12, are executed for each bin $k$ of the DFT; however, when several bins are calculated, the result of these operations/memory might be reused for 


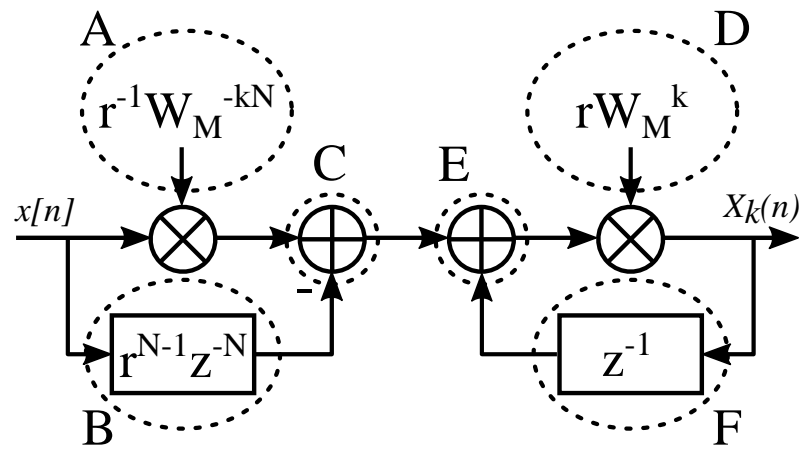

Figure 5.12: Modified and stabilized SB-SDFT filter with reduced multiplication

multiple bins i.e. that part of the block can be shared between a few bins. This is further explained in the following analysis wherever applicable.

\subsubsection{COMplex Operations}

The complexity of the proposed method is separately calculated for the zoom and the window alignment stage while explaining the operations shown by the dotted circles of Figure 5.12. Each time that a new value for the DFT bin is calculated is called an iteration. Each iteration updates the DFT value based on the input sample and the last $N-1$ samples of the input sequence (in total $N$ samples). In the following, multiplication and addition refer to complex operations unless stated otherwise.

\section{Zoom Stage:}

A: The twiddle factor in $A$ for the last step $\gamma=\Gamma$ is $r^{-1} W_{N I}^{-k N}$ for bin $k$ which is written as $r^{-1} W_{I}^{-k}$. Since $W_{I}=\exp (j 2 \pi / I)$ is always a point on the unit circle within the complex plane, its powers have only $I$ different values regardless of the number of calculated bins. Since $r^{-1} W_{I}^{-(k+I / 2)}=-r^{-1} W_{I}^{-k}$, only half of the twiddle factor multiplications, $0.5 I$, need to be calculated and the rest are simply sign conversions (multiplications with $+/-j$ are still considered as a complete complex multiplication). The last step of the zoom stage has the largest zeropadding factor and consequently, the largest number of different twiddle factors. So the number of multiplications within operation $A$ is the largest in the last step. To simplify the complexity expressions we consider $0.5 \mathrm{I}$ multiplications for the other steps of the zoom stage as well. So $A$ leads to $(0.5 I)(\Gamma+1)$ multiplications per iteration for all bins and all steps together.

B: The operation in $B$ is a real-by-complex multiplication and we approximate it by 0.5 complex multiplication. It can be shared between all bins at each step. For all steps together, it leads to $0.5(\Gamma+1)$ multiplications for each iteration and all bins together. 
$\mathrm{C}$ : Based on the above discussion, the output of $A$ may have at most $I$ different values in each step for all bins and $B$ can be shared with all bins. As a result, $C$, leads to $I$ additions (at most) per iteration in each step for all bins together. So $C$ leads to $I(\Gamma+1)$ additions per iteration for all bins and all steps together.

$\mathrm{D} / \mathrm{E}$ : These parts together include one multiplication and one addition per bin and per iteration. In the zoom stage, at the first step, $N$ bins are calculated and for the other $\Gamma$ steps $I$ bins are calculated $(N+I \Gamma$ in total). Thus, for the zoom stage, per iteration and for all bins together $D$ and $E$ lead to $N+I \Gamma$ multiplications and additions, respectively.

Iterations: remember that for each step of the zoom stage the window shifts $2 N$ samples (i.e. $2 N$ iterations). Starting from the initial state of zero, $N$ iterations are required to generate the DFT value for the first window of $N$ samples. Then, each iteration gives the SB-SDFT for the next window. As a result, there are $3 N$ iterations for each bin at each step of the zoom stage. Now, considering the above discussion and the number of iterations, the total number of multiplications and additions for the zoom stage $\left(C M_{Z_{o o m}}\right.$ and $C A_{Z o o m}$, respectively) are obtained as follows.

$$
\begin{gathered}
C M_{\text {Zoom }}=3 N(0.5(I+1)(\Gamma+1)+(N+I \Gamma)) \\
C A_{\text {Zoom }}=3 N(I(\Gamma+1)+(N+I \Gamma))
\end{gathered}
$$

\section{Window Alignment Stage:}

A: In the window alignment stage, the zero-padding factor is $I$. Following the same reasoning as the zoom stage, $A$ needs $0.5 I$ multiplications per iteration for all bins together.

B: In total, this part needs two real multiplications or 0.5 complex multiplication per iteration for all bins together.

C: Similar to what was mentioned for the zoom stage, $C$ leads to $I$ complex additions per iteration for all bins together.

D/E: In the window alignment stage, $\left|B o I_{\text {Final }}\right|$ bins are calculated. Thus, per iteration and for all bins together $D$ and $E$ lead to $\left|B o I_{\text {Final }}\right|$ multiplications and additions, respectively.

Iterations: The window alignment stage needs $N(L-1)$ iterations where $L$ is the length of the preamble ${ }^{2}$. Following the same reasoning as for the zoom stage, $N$ extra iterations are required when starting from the initial state of zero so the alignment stage needs $N L$ iterations for each bin. The number of multiplications and additions for the window alignment stage $\left(C M_{\text {Align }}\right.$ and $C A_{\text {Align }}$, respectively) are:

\footnotetext{
${ }^{2} N$ delays for each symbol, from symbol 1 to symbol $L-1$ of the preamble.
} 


$$
\begin{gathered}
C M_{\text {Align }}=N L\left(0.5(I+1)+\left|B o I_{\text {Final }}\right|\right) \\
C A_{\text {Align }}=N L\left(I+\left|B o I_{\text {Final }}\right|\right)
\end{gathered}
$$

\subsubsection{MEMORY}

Since the zoom stage and the window alignment stage are not executed in parallel, the registers (memory elements) used in one can be used in the other as well. Here, we only focus on the window alignment stage as it needs more memory and therefore, dictates the total required memory for calculating the SDFT. For the Sliding DFT each filter needs a memory of size $N$ for samples and a delay of one for the previous DFT value (See $B$ and $F$ in Figure 5.11). The memory within $B\left(z^{-N}\right.$ with length $\left.N\right)$ can be shared between all bins and the second is unique for each bin. Additionally, the delay block in Figure 5.6 requires storing $2 N(\Gamma+1)$ samples. All these values have two parts (real and imaginary), so the required memory for calculating $\operatorname{SDFT}\left(M_{\text {Calc }}\right)$ is as follows.

$$
M_{\text {Calc }}=2\left(N+\left|B o I_{\text {Final }}\right|+2 N(\Gamma+1)\right)
$$

where $\left|B o I_{\text {Final }}\right|$ is the cardinality of the set of $B o I$ used in the window alignment stage. The proposed synchronization algorithm needs to store the results of the accumulation of the DFT magnitudes for $\left|B o I_{\text {Final }}\right|$ bins and $N$ delay values. This memory is denoted by $M_{A c c}$ and is derived as follows.

$$
M_{A c c}=2\left|B o I_{\text {Final }}\right| N
$$

The factor two comes from the two sets of magnitudes that must be accumulated for odd and even symbols.

For an $N I$-point DFT, $W_{N I}=\exp \left(\frac{j 2 \pi}{N I}\right)$ which means that $W_{N I}^{-k}$ takes $N I$ different values. Since $W_{N I}^{-(k+N I / 2)}=-W_{N I}^{-k}$, only half of these values should be stored from which two values are equal to 1 and $j$ for $k=0$ and $k=N I / 4$, respectively. As a result, $N I / 2-2$ complex twiddle factors should be stored so the required memory is $N I-4$.

\subsubsection{COMPARISON}

As mentioned in section 5.3, the SFFT method in [30] provides an efficient implementation of the Hara synchronization algorithm. The complexity of the SFFT for each iteration can be found in [30]. Due to zero-padding, in a few first stages of the SFFT some operations have zero inputs and are not executed which leads to a pruned structure with reduced complexity. The number of iterations for the SFFT in the Hara synchronization algorithm can be derived based on a reasoning similar to what was used above for the window alignment stage. Taking these into account, the complexity of the Hara synchronization 
can be calculated. The detailed calculations are included in Appendix B and only the final results are presented here for comparison.

The number of operations and memory required for the Hara synchronization and the proposed synchronization algorithms are presented in Table 5.2. For the total number of operations only the term with the largest power of $N$ is included in the table. Although the number of CM/CA increases with $N^{2}$ for both methods, in practical cases, the factor of $N^{2}$ in the Hara method is much larger than that of the proposed method. Considering $L=16$ and $I=8$, the factor of $N^{2}$ in the number of complex multiplications required for the Hara synchronization (implemented using SFFT) is more than 40 times the factor of $N^{2}$ in the complexity of the proposed synchronization algorithm. Furthermore, the total memory of the Hara method (with SFFT) increases with $N^{2}$ while the total memory of the proposed method increases with $N$. These differences between the complexity stem from the fact that in the proposed synchronization algorithm, in contrast with the Hara synchronization, the number of the calculated DFT bins for the window alignment does not change with $N$ and is constant $\left(B o I_{\text {Final }}\right)$. The numerical results and comparison of complexity for different values of $N$ are presented in the next section.

\subsection{Simulation Results and Discussion}

\subsubsection{Design Parameters}

According to our discussions in the previous sections, two parameters need yet to be determined. Those are the number of bins in the BoI Final and the damping factor $r$ for the SB-SDFT. The most important performance metric for our system is the overall BER of the demodulator. Therefore, BER values obtained by simulations are utilized to determine the appropriate design parameters. As stated in 5.4 , in all simulations of this section $I=8$ and $L=16$ similar to Chapter 3. Figure 5.13 shows how the BER of the demodulator changes relative to $\mid$ BoI Final $\mid$ for different values of $E_{b} / N_{0}$. The damping factor is considered to be $r=1$ in these simulations. As can be seen in Figure 5.13, the bit error rate value hardly changes when the number of bins in the $B o I_{F i n a l}$ is increased to more than 14. For a safety margin the number of bins in the BoI Final is considered to be 16 .

The value of the damping factor determines the error at the output of the SBSDFT filter. As mentioned earlier, the damping factor introduces errors to the calculated values for the DFT which may degrade the performance of the demodulator. This can be solved by choosing $r$ very close to one. Additionally, the number of samples which pass through the SB-SDFT filter plays an important role in the value of $r$. Due to the recursive behavior of the filter, the error accumulation increases when an SB-SDFT is applied to a long sequence of samples. Thus, for a constant preamble length, the appropriate value for $r$ also depends on the number of samples per symbol. As explained in Chapter $3, N$ is propor- 


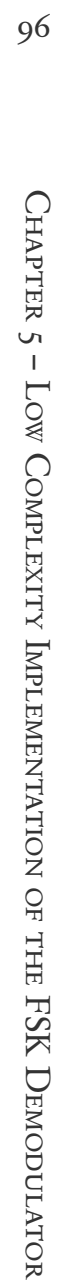

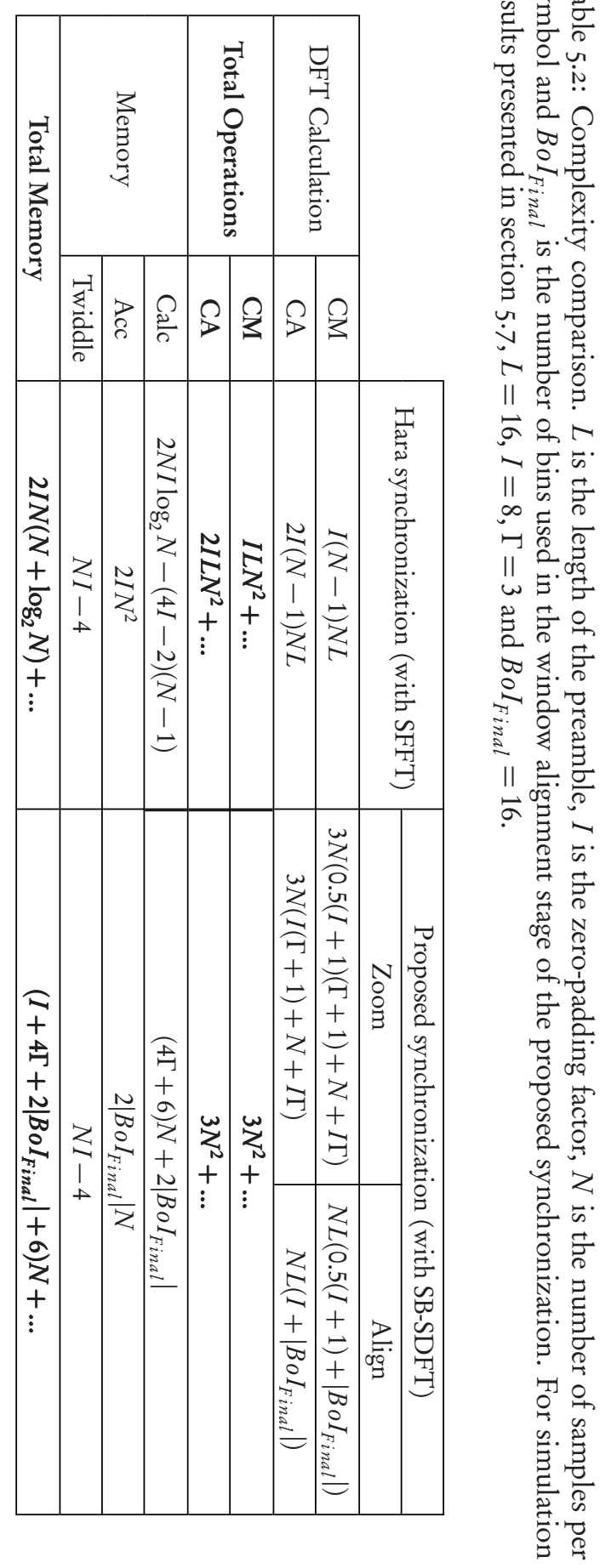




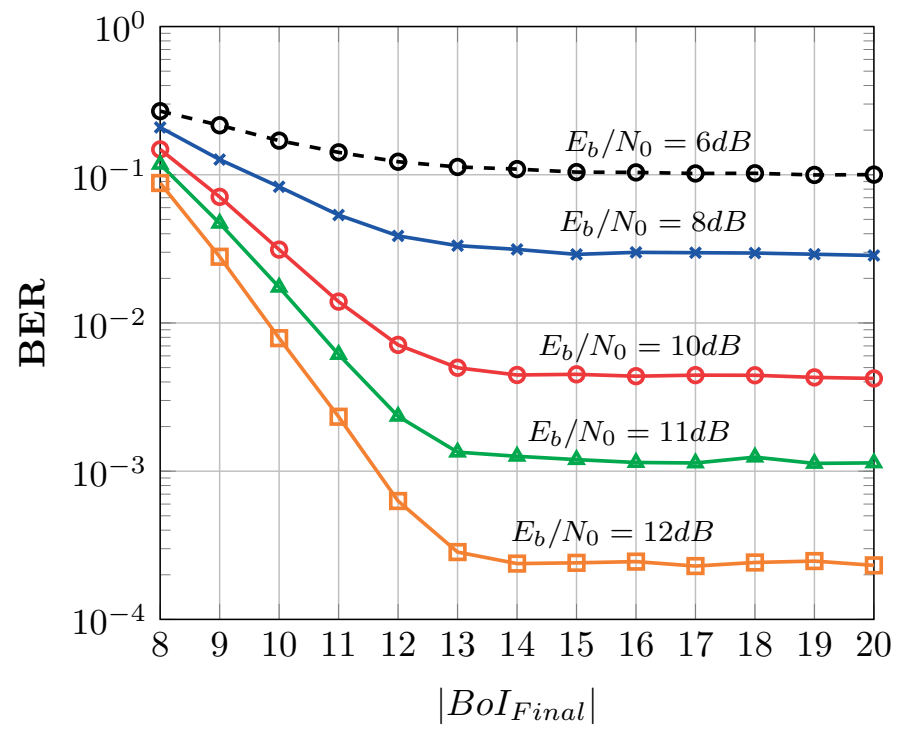

Figure 5.13: The BER values for the demodulator, using the proposed synchronization and the proposed SB-SDFT, for different sizes of the BoI Final when $N=I=8, L=16$ and $r=1$.

tional to the bandwidth of the filter before the demodulator so it determines the tolerable frequency offset. Figure 5.14 illustrates the BER at $E_{b} / N_{0}=11 d B$ (corresponding to $B E R \approx 0.1 \%$ ) for different values of $N$ and $r$ when $L=16$ and $I=8$. According to Figure 5.14, $r=0.999$ guarantees a consistent performance up to a sampling frequency more than 100 times the symbol rate.

\subsubsection{BER PERformance}

To evaluate the BER performance and compare it with the conventional algorithm (Hara synchronization), the proposed algorithm is applied to a DFT-based demodulator with parameters similar to [89]. The sampling frequency is $8 R_{S y m}$ where $R_{\text {Sym }}$ is the symbol rate while $I=8$. The total system is simulated for an AWGN channel. It is assumed that the samples are the output of a brick-wall low-pass filter so the noise samples are uncorrelated.

The BER performance of a DFT-based demodulator using the proposed synchronization and the Hara synchronization ([89]) algorithms is depicted in Figure 5.15. As can be seen, the performance of the proposed method is only slightly worse at high BER values; however, for practical BER values in the order of $10^{-3}$ or smaller, it performs the same (within a range $<0.1 \mathrm{~dB}$ ) as the Hara method.

Figure 5.16 illustrates the BER for a range of frequency offsets values up to twice the symbol rate and different $E_{b} / N_{0}$. It can be seen that the demodulator with the 


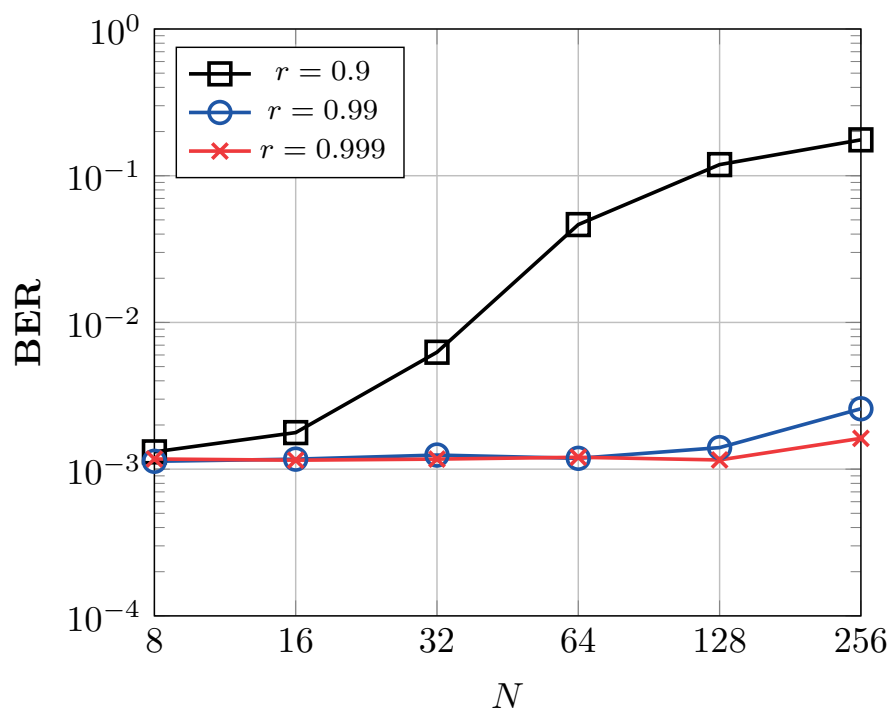

Figure 5.14: The BER of the demodulator with the proposed synchronization which is implemented using the proposed stable SB-SDFT. $E b / N_{0}=11 \mathrm{~dB}$, $I=8$ and $L=16$.

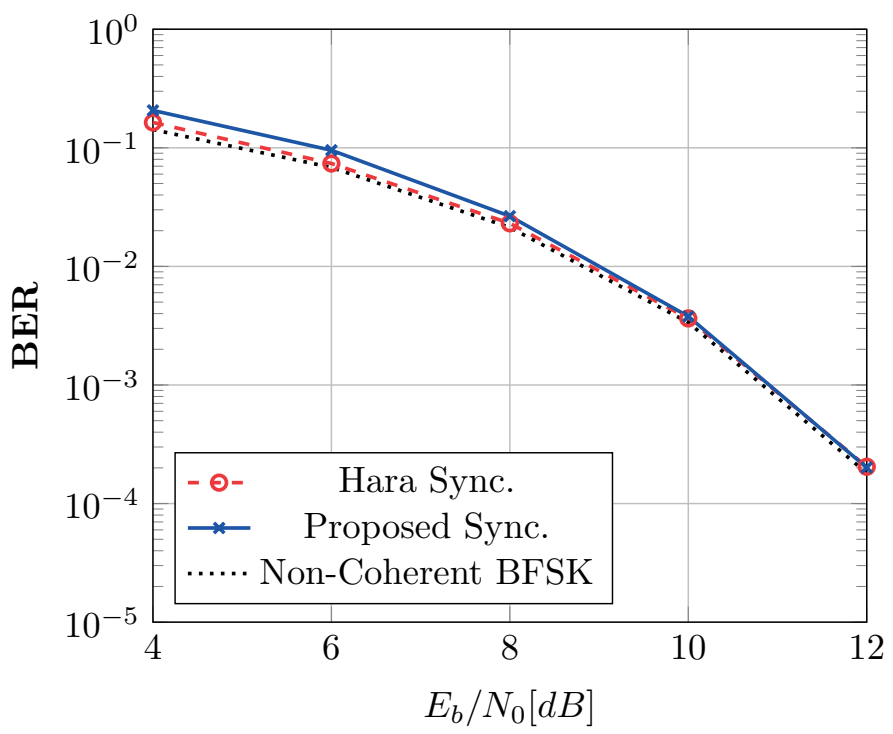

Figure 5.15: The BER curves for the DFT-based demodulator in Chapter 3 with the proposed synchronization and Hara synchronization. 


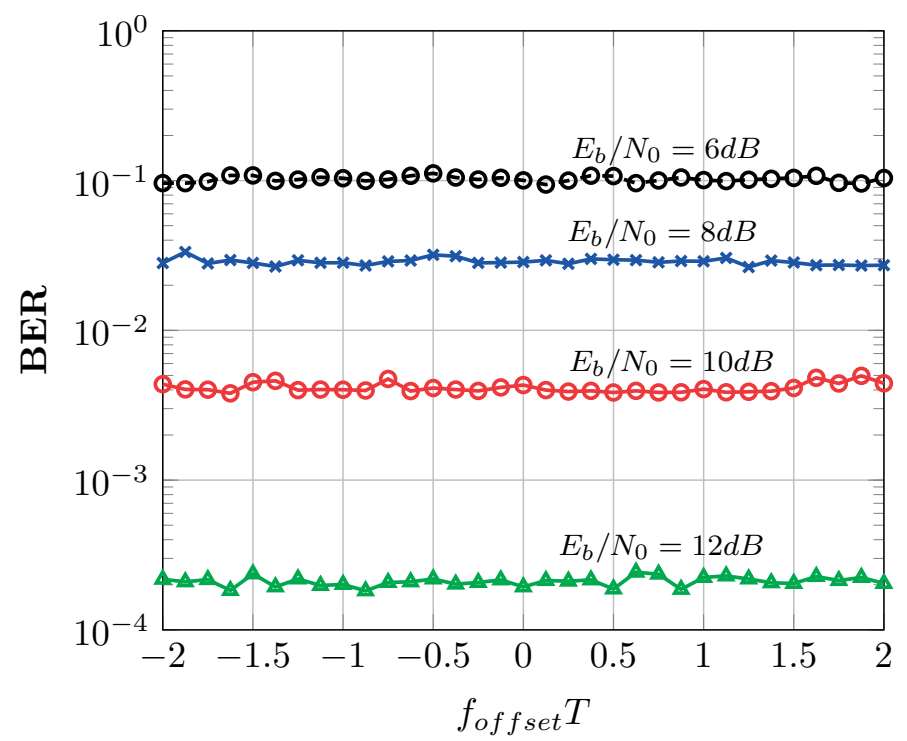

Figure 5.16: The BER of the demodulator with the proposed synchronization algorithm for different frequency offsets normalized to the symbol rate $\left(f_{\text {of } f \text { set }} T\right)$.

proposed synchronization algorithm can tolerate frequency offset as expected.

Figure 5.17 shows the BER performance of the proposed method for different values of $N$ and $E_{b} / N_{0}$. For these simulations $I=8, L=16$ and $r=0.999$. It is seen that the increase of $N$ does not affect the BER performance of the demodulator using the proposed synchronization algorithm and the proposed SB-SDFT. Notice that increasing $N$ means that the bandwidth of the filter prior to the demodulator increases (see discussion in Chapter 3 ). This means that the noise bandwidth increases. As can be seen in the results, this increased noise bandwidth does not degrade the BER performance. This can be justified by looking at the DFT as a bank of narrowband filters as discussed in Chapter 3.

Finally, Figure 5.18 illustrates the performance of the demodulator with the proposed synchronization in Rayleigh and Rician fading $(K=4)$ channels. It can be seen that a demodulator using the proposed synchronization performs the same as a demodulator with Hara synchronization algorithm.

\section{$5 \cdot 7 \cdot 3$ Complexity}

Figure 5.19 shows the number of complex operations and memory required for both methods and different values of $N$ while $I=8,\left|B o I_{\text {Final }}\right|=16$ and $L=16$ (both axes are on logarithmic scale). Furthermore, Figure 5.20 shows the improvement achieved using the proposed algorithm and SB-SDFT. The improvement is defined as the percentage of the reduced operations relative to 


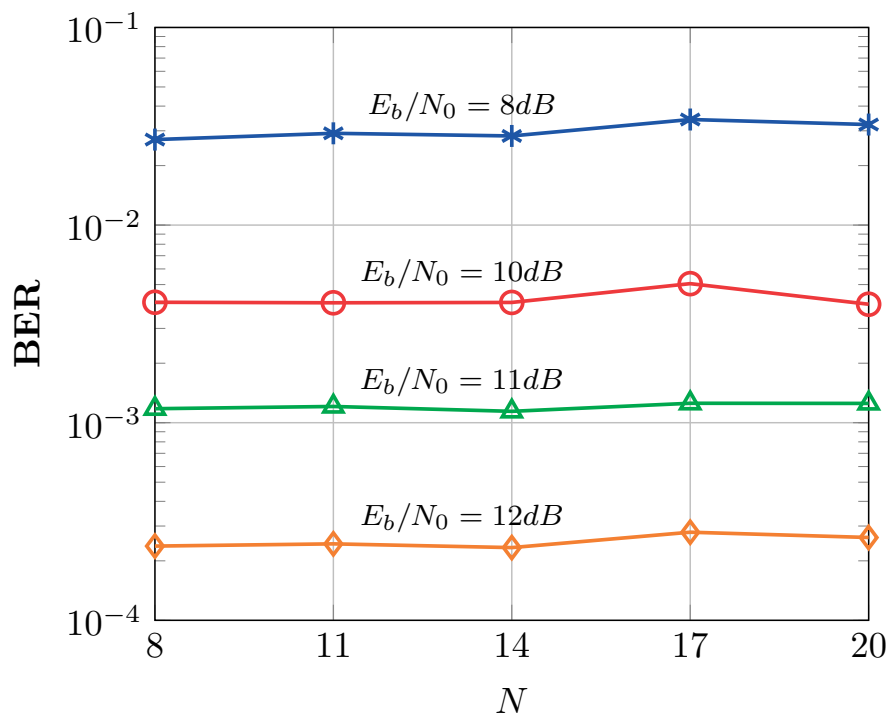

Figure 5.17: The BER of the demodulator with the proposed synchronization for different values of $N$ and $E_{b} / N_{0}$ while $I=8, L=16$ and $r=0.999$.

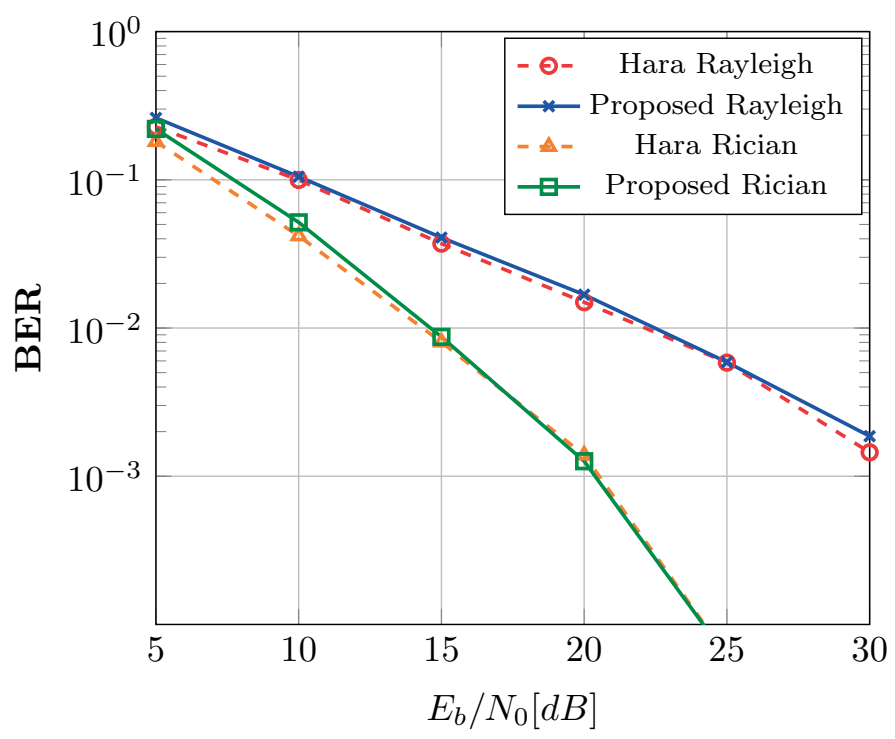

Figure 5.18: The BER curves for the DFT-based demodulator with the proposed synchronization and Hara synchronization in a Rayleigh and a Rician $(\mathrm{K}=4)$ channel. In this simulations $N=8, I=8, L=16$ and $r=0.999$. 
the number of operations in the Hara method implemented using the SFFT (e.g. $\left.100 \% \times\left(C M_{\text {Hara }}-C M_{\text {Proposed }}\right) / C M_{\text {Hara }}\right)$. Notice that the values of $N$ are selected to be a power of two as required by the SFFT implementation of the Hara method; however, this is not necessary for the efficient implementation of the proposed algorithm. For $N=8$, which was used to achieve BER curves, the proposed method reduces the number of complex additions, complex multiplications and memory by $68 \%, 47 \%$ and $61 \%$, respectively (compared to the efficient implementation of the Hara algorithm).

Figure 5.19 and Figure 5.20 show that the difference between the complexity of the proposed method (with SB-SDFT) and the Hara algorithm (with SFFT) increases when $N$ increases. One of the reasons is that only the number of DFT bins calculated in the first step of the zoom stage depends on $N$. For the next steps of the zoom stage and the alignment stage, the number of the calculated bins is constant for all $N$ ( $I$ and $\left|B o I_{\text {Final }}\right|$, respectively). Although the number of samples involved in the DFT calculation is still related to $N$, the complexity grows slower as the number of bins required to be calculated remains constant. That is why the difference between the complexity of the proposed synchronization and the Hara synchronization increases when $N$ increases. For a sampling frequency which is 32 times the symbol rate, almost $85 \%$ saving can be achieved in arithmetic operations (and around $90 \%$ in the memory). The nullto-null bandwidth of a BFSK modulated signal ( $B_{S}$ in Figure 3.10) for a frequency separation of $f_{\text {sep }}=R_{\text {Sym }}$ is $3 R_{\text {Sym }}$ [103]. If a very steep filter is considered $\left(B_{t}=\right.$ 0 in Figure3.10) such a sampling frequency means that a frequency offset around $\pm 14.5 R_{\text {Sym }}$ can be tolerated. As shown in Figure 5.15 and Figure 5.18, the DFTbased demodulator which uses the proposed synchronization implemented using SB-SDFT has a BER performance similar to the DFT-based demodulator with the Hara synchronization; thus, the proposed synchronization and its efficient implementation reduce the complexity without any sacrifice in the performance.

\subsection{CONCLUSion}

In this chapter, a new synchronization algorithm was proposed to decrease the complexity of the synchronization for the DFT-based FSK demodulator. Using a step-by-step zooming technique, the proposed algorithm only requires a subset of the DFT bins. Consequently, efficient Single Bin Sliding DFT (SB-SDFT) implementations can be used. Besides, a stable SB-SDFT was introduced to incorporate zero-padding. The proposed algorithm and its implementation using the SB-SDFT for an oversampling factor of e.g. $N=8$, obtains $47 \%, 68 \%, 61 \%$ saving in the number of complex multiplications, complex additions and memory usage compared to the conventional window synchronization algorithm (Hara synchronization) while achieving the same BER performance. The relative reduction in the complexity further increases when larger $N$ is required. The higher the sampling frequency (the larger value of $N$ ), the larger frequency offset can be tolerated. For a frequency offset tolerance about \pm 14.5 times the 


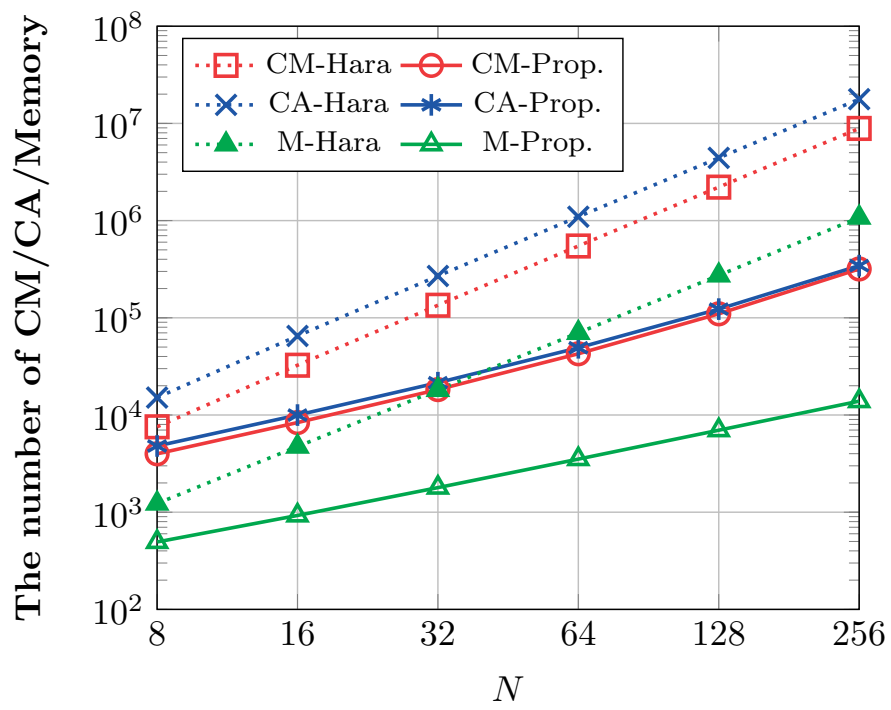

Figure 5.19: The complexity of Hara algorithm implemented using SFFT (shown by Hara) and the proposed algorithm implemented using the proposed SB-SDFT (shown by Prop.) in terms of complex additions (CA), complex multiplications $(\mathrm{CM})$ and memory (M).

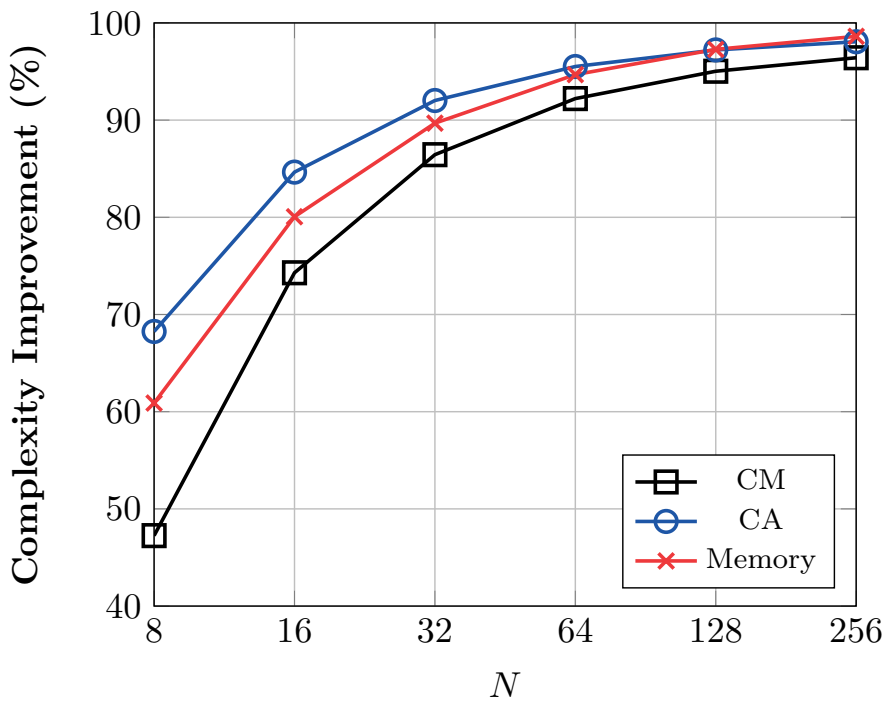

Figure 5.20: The improvement in complexity achieved by the proposed method implemented using the proposed SB-SDFT. 
symbol rate which requires an oversampling factor $N=32$, the number of complex operations is reduced by more than $85 \%$ (and memory by $90 \%$ ) compared to the conventional method (Hara synchronization implemented using SFFT). 
104 


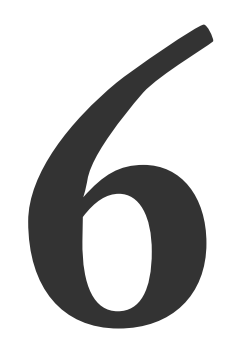

\title{
Hybrid Modulation AND TIME Diversity
}

\begin{abstract}
Carrier frequency offset (CFO) and time-varying fading channels are two problems that ultra-narrowband solutions for the emerging Low Power Wide Area Networks (LPWAN) need to tackle. To overcome CFO, offset tolerant demodulators have been proposed for both PSK and FSK modulations. To combat channel distortion, time or frequency diversity together with coding techniques can be utilized. To afford the redundancy required for these solutions, a lower effective bit-rate has to be accepted or a bigher bit-rate is required. Higher order PSK and FSK considerably compromise energy and bandwidth, respectively. As a third solution, hybrid modulation has been proposed which can increase the bit-rate in a more power and bandwidth efficient way than PSK and FSK, respectively. In this work, an offset tolerant demodulator is proposed for hybrid modulations combining Double Differential $M$-ary PSK (DDMPSK) and Differential L-ary FSK (DLFSK). The proposed demodulator is evaluated for different combinations of $M$ and $L$ in a timevarying channel using a time diversity technique. Through different scenarios the hybrid demodulator is compared to DDMPSK. The results demonstrate that at $B E R=0.1 \%$, the hybrid modulation can improve BER performance compared to higher order DDMPSK in all scenarios.
\end{abstract}

\subsection{INTRODUCTION}

In the previous chapters, offset tolerant demodulators were investigated to overcome Carrier Frequency Offset (CFO) in UNB communication systems. In addition to CFO, UNB systems are vulnerable to temporal fading due to their long symbol time. As shown in [71], for a low data rate system, a time-varying channel can even cause an error floor. As a consequence of time-varying behav-

This chapter is based on [S:1] and [S:5]. 
ior, channel estimation at the beginning of a packet is not reliable. Furthermore, using multiple pilot sequences for channel estimation imposes prohibitive overhead due to short packets used in these systems (as short as 200 symbols $[38,55]$ ).

In such circumstances, diversity techniques can be utilized to improve performance [71]. Space diversity requires multiple antennas and RF chains which makes it improper for a low power and area constrained receiver. Frequency diversity using techniques such as frequency hopping is another option. In a highly time-variant channel, time diversity can be achieved using channel coding and interleaving. In either of these two techniques redundant information must be transmitted.

As discussed in the previous chapters, offset tolerant demodulators have been proposed for both FSK and PSK modulations and they can be employed in UNB communication systems which are susceptible to CFO. However, transmitting redundant information increases either the time required to transmit a packet of information bits or the bit-rate. Longer transmission time, increases on-time of the $\mathrm{RF}$ front-end which plays a significant role in the overall power consumption. Therefore, a higher raw bit-rate is preferred. One solution is using higher order PSK. The downside of this solution is loss in the BER performance. As an alternative a higher order FSK can be employed which compromises bandwidth for a better BER performance. Hybrid frequency/phase modulation is a third possible solution which has been suggested in literature $[17,34,35,52,57,87]$.

In this chapter, an offset-tolerant modulator and demodulator for hybrid modulation is proposed to tackle frequency offset and temporal fading at the same time. The demodulator is designed for a combination of LFSK and MPSK. To get insight into the performance of the hybrid modulation, the demodulator is evaluated for various combinations of MPSK and LFSK while time-varying Rayleigh and Rician channel models are considered. Moreover, the bandwidth compromise of the hybrid modulation compared to PSK modulation is taken into account through investigating an equal bandwidth scenario.

The rest of this chapter is organized as follows. The next section reviews existing work on the hybrid modulation technique. Section 6.3 elaborates on the design of the hybrid demodulator in the general cases of MPSK and LFSK and its tolerable frequency offset. A mathematical formulation and error floor expressions are presented in section 6.4 to show how the proposed demodulator performs in a time-varying channel and why using FSK is better than solely using higher order PSK. The system including time diversity is designed and explicated in section 6.5. Simulation results and discussions for an AWGN channel and a time-varying channel with different scenarios are included in sections 6.6 and 6.7, respectively. Finally, section 6.8 concludes this chapter. 


\subsection{ReLATED WORK}

As mentioned in Chapter 1 and Chapter 2, the hybrid frequency/phase modulation has been investigated as an alternative option to change the power and bandwidth efficiency trade-off of higher order PSK and FSK. A combination of LFSK and MPSK can benefit from the energy efficiency of higher order FSK while it alleviates the LFSK spectral deficiency with higher order PSK.

One of the early examples of frequency/phase modulation (FPSK) was proposed in [87]. This idea was later formulated in $[59,93]$ as an L-orthogonal signaling method where both frequency and phase are modulated. For FPSK signals to be orthogonal for any order of phase modulation, the frequency separation should be equal to the symbol rate [34,93]. In [52], a signal is generated by combining $N$ different frequencies each of which is modulated using one of the $M$ possible phases of MPSK and a coherent receiver for such modulation scheme is proposed. Using various combinations of frequency tones and phase modulation, $M^{N}$ different modulated signals can be generated. For higher energy efficiency, a modification to the proposed modulation is introduced called expurgated phase codes. It only uses combinations which have minimum correlation [52]. This modified version decreases bandwidth efficiency but improves the BER performance. Using only half of the code combinations (E4-FPSK) the modified version can achieve more than $4 \mathrm{~dB}$ improvement compared to 8PSK which provides the same modulation order. The bandwidth efficiency is though decreased by $30 \%$.

In [34], a non-coherent demodulator for a frequency/phase modulation scheme is proposed using non-coherent FSK and Differential PSK (DPSK). During each symbol, the frequency is modulated with one of the L possible tones of LFSK and phase is differentially modulated with MPSK. It is shown that using a combination of FSK and DPSK requires lower $E_{b} / N_{0}$ compared to higher order PSK when achieving the same BER performance. For instance, using a combination of BFSK and QPSK instead of 8PSK, which provides the same modulation order, achieves almost $6 \mathrm{~dB}$ energy saving at $\mathrm{BER}=0.1 \%$ [34]. This improvement is achieved at the cost of $30 \%$ decrease in bandwidth efficiency. The performance of FPSK in slow and flat fading channels is investigated in [35]. It shows that, in a flat and slow Rayleigh fading channel, FPSK is still better than higher order PSK but the improvement achieved in the BER performance is less compared to what is achieved in an AWGN channel.

Despite all the work in literature, no offset tolerant demodulator has been designed for the FPSK modulation scheme capable of tolerating CFO which is much larger than the symbol rate. Furthermore, the performance of FPSK in a time-varying channel has not been investigated. In this chapter, an offset tolerant demodulator is designed for the frequency/phase modulation (FPSK) and its performance in a time-varying channel using a time diversity scheme is evaluated. The next section elaborates on the design of the modulator, demodulator and 
Table 6.1: Parameters and notation

\begin{tabular}{|c|c|}
\hline FSK order & $L$ \\
\hline PSK order & $M$ \\
\hline Symbol rate & $R_{S}$ \\
\hline Bit-rate & $R_{b}$ \\
\hline Energy per symbol & $E_{S}$ \\
\hline Energy per bit & $E_{b}$ \\
\hline Energy per information bit & $E_{b}^{I n f}$ \\
\hline Sampling time & $T_{s}$ \\
\hline Sampling frequency & $F_{s}$ \\
\hline Symbol duration & $T$ \\
\hline FSK frequency separation & $f_{s e p}$ \\
\hline$n^{t h}$ symbol modulated frequency & $\omega_{n}=2 \pi f_{n}$ \\
\hline$n^{t h}$ symbol modulated phase & $\varphi_{n}$ \\
\hline Packet length & $L_{P}$ \\
\hline Frequency offset & $\omega_{O}=2 \pi f_{O}$ \\
\hline Samples per symbol & $N$ \\
\hline
\end{tabular}

the range of tolerable frequency offset.

\subsection{Offeset Tolerant Hybrid Demodulator}

The proposed offset tolerant demodulator makes it possible to employ hybrid modulation in applications which are susceptible to large frequency offset (multiple times the symbol rate). To explain offset tolerant demodulator design, first the hybrid modulator is explained. Then, an offset tolerant demodulator for this modulation is achieved and the range of frequency offset that can be tolerated is analyzed.

\subsubsection{Modulator Design}

For offset tolerant hybrid modulation, a combination of Differential LFSK (DLFSK) and Double Differential MPSK (DDMPSK) is utilized. These techniques and the reason for choosing differential and double differential encoding will be clarified later when the demodulator is derived. The parameters and symbols used in the signal model are summarized in Table 6.1.

A hybrid modulator modulates both phase and frequency based on the transmitted bits. The block diagram of the proposed modulator is shown in Figure 6.1a. The modulator receives two sets of bits; $B_{F S K}$ is the set of bits modulated over 
FSK and $B_{P S K}$ is the set of bits modulated using PSK. In the upper branch, a simple MPSK modulator and double differential encoder are used. First, the bits are mapped to symbols to achieve $\alpha_{n}=2 \pi \mathrm{m} / M$ which is the modulated phase for the $n^{t h}$ symbol and $m=0, \ldots, M-1$. Then, mapped symbols go through two cascaded differential encoders to generate $e^{j \beta_{n}}$ and $e^{j \varphi_{n}}$, respectively. $e^{j \varphi_{n}}$ is the phase of transmitted DDMPSK signal during the $n^{\text {th }}$ symbol. In each differential encoder the modulated MPSK signal is multiplied with its delayed version (one symbol period delay). The relations between $\alpha, \beta$ and $\varphi$ are as follows.

$$
\begin{aligned}
& \alpha_{n}=\beta_{n}-\beta_{n-1} \\
& \beta_{n}=\varphi_{n}-\varphi_{n-1}
\end{aligned}
$$

In the lower branch, bits are mapped to integers (symbols), $\gamma_{n}$, which correspond to the frequency indexes of an LFSK modulation; $\gamma_{n} \in 0, \ldots, L-1$. In the LFSK differential demodulator (explained later) the difference between two consecutive symbol frequencies is detected; thus, the symbols should be coded into the difference of consecutive symbol frequencies. For this purpose a differential encoder is applied to the frequency index $\gamma_{n}$ to generate the final transmitted frequency index as follows.

$$
\lambda_{n}=\left(\gamma_{n}+\lambda_{n-1}\right) \bmod (L)
$$

In (6.2), $(x) \bmod (L)$ shows the remainder of $x$ divided by $L$. When the index is determined, the frequency of LFSK during the $n^{\text {th }}$ symbol period is obtained as follows.

$$
f_{n}=-f_{\text {sep }} / 2+\left(\lambda_{n}+1-L / 2\right) f_{\text {sep }}
$$

where $f_{\text {sep }}$ is the FSK frequency separation. Using (6.1) and (6.3), the baseband equivalent of the transmitted signal during the $n^{\text {th }}$ symbol, $s(t)$, is as follows.

$$
s(t)=\sqrt{E_{S} / T} \exp \left[j\left(\omega_{n} t+\varphi_{n}+\theta_{n}\right)\right],(n-1) T \leq t<n T
$$

where $2 \pi f_{n}$ is replaced by $\omega_{n}$ for easier notation and $\theta_{n}$ is the initial phase at the beginning of the $n^{\text {th }}$ symbol including a constant phase offset. Similar to other frequency/phase modulation schemes, the frequency separation of LFSK is considered to be equal to the symbol rate to guarantee orthogonality [34].

\subsubsection{Demodulator Design}

To derive the proposed offset tolerant demodulator for the hybrid modulation, we start with the samples of the signal at the input of the demodulator. Here, we assume that the sampling frequency $F_{s}=N R_{S}$, is an integer multiple of the 


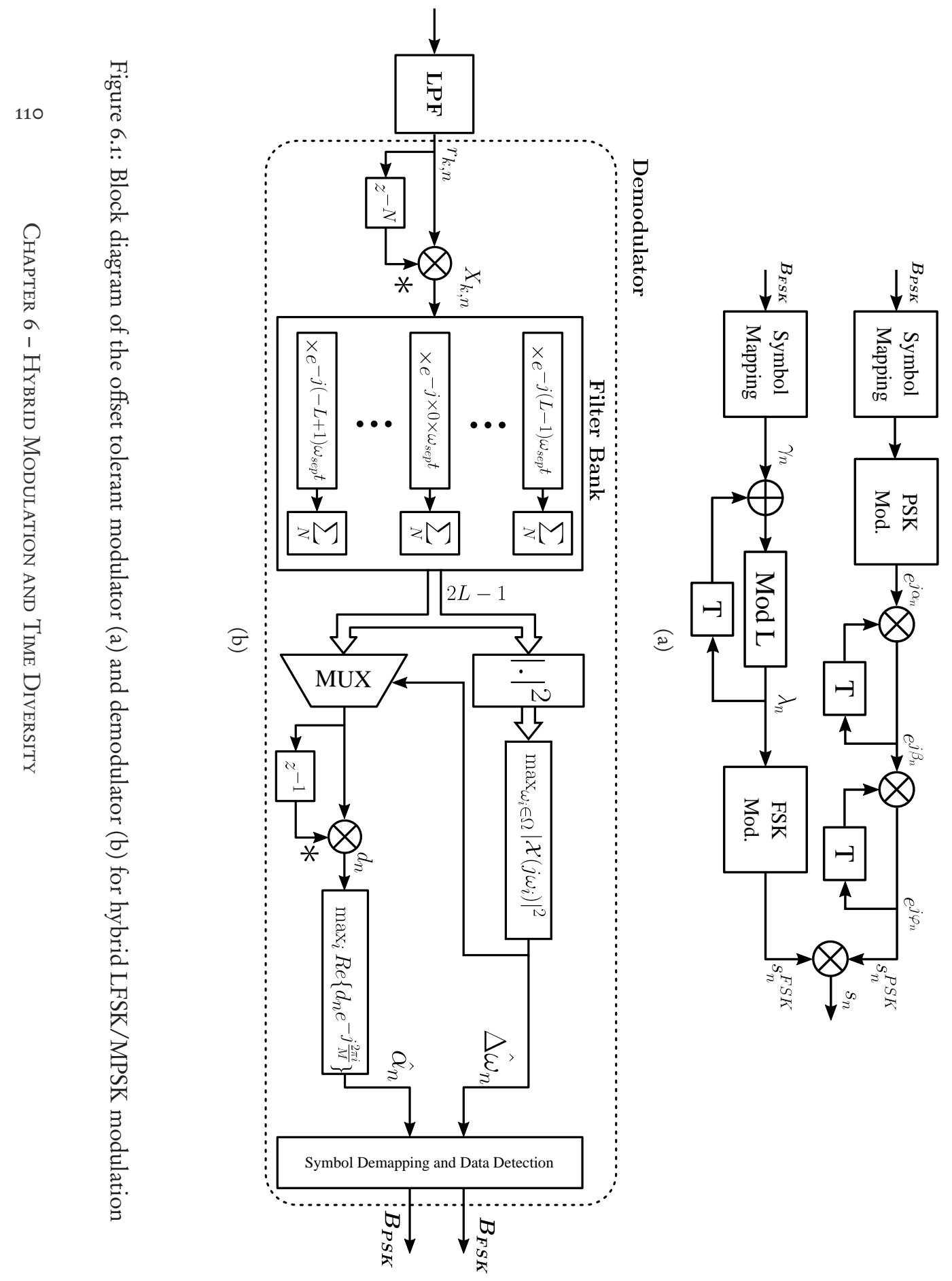


symbol rate $R_{S}$, and $N$ is the number of samples per symbol. The effect of the sampling frequency on the offset tolerance is elaborated later; but for now, it is assumed that $F_{s}$ is large enough to meet the Nyquist criterion even in the presence of frequency offset.

Considering (6.4), the input samples during the $n^{\text {th }}$ symbol (assuming no noise) are as follows:

$$
r_{k, n}=\sqrt{E_{S} / N} \exp \left[j\left(\left(\omega_{n}+\omega_{O}\right)\left((n-1) T+k T_{s}\right)+\varphi_{n}+\theta_{n}\right)\right]
$$

where $r_{k, n}$ is the $k^{t h}$ sample of the $n^{t h} \operatorname{symbol}(k=1, \ldots, N)$ and the rest of the parameters are as specified in Table 6.1.

The block diagram of the proposed demodulator is shown in Figure 6.1b. Samples pass through a Low-Pass Filter (LPF) before the demodulator which is wide enough to include the signal in the presence of frequency offset. The LPF is not part of the demodulator; nevertheless, it is shown in the figure for future reference. In our analysis, the effect of the filter is only considered as changing the noise bandwidth and it is assumed that this filter does not cause any ISI as indicated before.

Before describing the details of the block diagram, the demodulator is derived mathematically. In the first step, an autocorrelation demodulator similar to the one used for DDMPSK [94] is applied to the signal (see DDPSK demodulator in Chapter 3). In this step, the samples of each symbol are multiplied by the conjugate of the samples from the previous symbol. The samples after multiplication, $X_{k, n}$, are as follows.

$$
X_{k, n}=r_{k, n} r_{k, n-1}^{*}=\frac{E_{S}}{N} \exp \left[j\left(\Delta \omega_{n} k T_{s}+\Theta_{n}\right)\right]
$$

where $X_{k, n}$ is the $k^{t h}$ sample of $X_{n}$ after multiplication when the $n^{\text {th }}$ and the $(n-1)^{t h}$ symbols are considered. $\Theta_{n}$ is as follows.

$$
\Theta_{n}=\left(\omega_{n}+\omega_{O}\right) T+\Delta \omega_{n}(n-2) T+\Delta \varphi_{n}+\Delta \theta_{n},
$$

where $\Delta \omega_{n}=\left(\omega_{n}-\omega_{n-1}\right)=2 \pi\left(f_{n}-f_{n-1}\right)$ is the difference between frequencies of two consecutive symbols and $\Delta \varphi_{n}=\varphi_{n}-\varphi_{n-1}$ is the difference between the information bearing phase of the signal in these two symbols. $\Delta \theta_{n}=\theta_{n}-\theta_{n-1}$ is the integral of the frequency during the $(n-1)^{t h}$ symbol; thus, $\Delta \theta_{n}=\left(\omega_{n-1}+\right.$ $\left.\omega_{O}\right) T$. So $\Theta_{n}$ can be rewritten as:

$$
\Theta_{n}=\left(\omega_{n}+\omega_{n-1}+2 \omega_{O}\right) T+\Delta \omega_{n}(n-2) T+\Delta \varphi_{n},
$$

Replacing $\omega_{n}$ and $\omega_{\text {sep }}$, respectively, with $2 \pi f_{n}$ and $2 \pi f_{\text {sep }}$ in (6.3), it can be seen that $\omega_{n}-\omega_{n-1}=\left(\lambda_{n}-\lambda_{n-1}\right) \omega_{\text {sep }}$. Using (6.2) and modular arithmetic, the relation between $\lambda_{n}$ and $\gamma_{n}$ can be written as follows. 


$$
\lambda_{n} \equiv \gamma_{n}+\lambda_{n-1}(\bmod L)
$$

$$
\gamma_{n} \equiv \lambda_{n}-\lambda_{n-1}(\bmod L)
$$

Since $0 \leq \gamma_{n}<L-1, \gamma_{n}$ can be achieved by calculating $\left(\lambda_{n}-\lambda_{n-1}\right) \bmod (L)$. Considering LFSK modulation as designed earlier, $\Delta \omega_{n}$ is an integer multiple of $\omega_{\text {sep }}$ and it can have $2 L-1$ different values in the set $\Omega=\left\{-(L-1) \omega_{\text {sep }}, \ldots, 0, \ldots,(L-\right.$ 1) $\left.\omega_{\text {sep }}\right\}$. From (6.6), it can be seen that the frequency component of $X_{k, n}$ only depends on FSK modulated data but not on the $\mathrm{CFO}\left(\omega_{O}\right)$.

Remember that in the designed modulator $\omega_{\text {sep }}=2 \pi / T$; thus, the frequencies in $\Omega$ are all multiples of $2 \pi / T, 2 \pi R_{S}$. If the Discrete Fourier Transform (DFT) is calculated for $N$ samples of $X_{n}$ during one symbol period (i.e. $X_{k, n}$ for $k=$ $1, \ldots, N$ ), the DFT bin resolution is $F_{s} / N=R_{S}$ (the box shown as Filter Bank in Figure $6.1 \mathrm{~b}$ calculates the required DFT bins). Hence, the information bearing frequency $\left(\Delta \omega_{n}\right)$ can be obtained using the Discrete Fourier Transform (DFT) of $X_{n}$ as follows [44].

$$
\Delta \hat{\omega}_{n}=\underset{\omega_{i} \in \Omega}{\arg \max }\left|\mathscr{X}\left(\omega_{i}\right)\right|^{2},
$$

where $\Omega=\left\{-(L-1) \omega_{\text {sep }}, \ldots, 0, \ldots,(L-1) \omega_{\text {sep }}\right\}$ is the set of possible values of $\Delta \omega_{n}$ for LFSK and $\mathscr{X}\left(\omega_{i}\right)$ is the Discrete Fourier Transform of $X_{n}$. To proceed, the value of $\Delta \hat{\omega}_{n}$ is substituted in the definition of the DFT for $X_{n}$ :

$$
\begin{aligned}
\mathscr{X}_{n}\left(j \Delta \hat{\omega}_{n}\right) & =\sum_{k=0}^{N-1} X_{k, n} \exp \left[-j \Delta \hat{\omega}_{n} k T_{s}\right] \\
& =E_{S} \exp \left[j \Theta_{n}\right],
\end{aligned}
$$

where $\Theta_{n}$ is calculated by (6.8). Each possible value of $\Delta \omega_{n}$ is exactly equal to one of the DFT bins (and not a value between them). As a result, the frequency component of $X_{n}, \Delta \omega_{n}$, is completely removed in (6.12) and the phase component can be used for further analysis without any distortion. Notice that this would not have been possible if the frequency of $X_{n}$ had not been exactly on a specific DFT bin.

After determining $\Delta \omega_{n}$ and removing the frequency, it is time to extract the information encoded in the phase of the signal. The residual phase component $\left(\Theta_{n}\right)$ includes two different parts (see (6.8)), one depends on the modulated phase of two adjacent symbols $\left(\Delta \varphi_{n}\right)$ and the other depends on the current and the previous symbol frequency and the $\mathrm{CFO}\left(\left(\omega_{n}+\omega_{n-1}+2 \omega_{O}\right) T+\Delta \omega_{n}(n-2) T\right)$.

As mentioned before, the frequency separation in the LFSK is equal to the symbol rate; $f_{\text {sep }}=1 / T$. Consequently, the term $\Delta \omega_{n}(n-2) T$ in (6.8) is 
always an integer multiple of $2 \pi$; thus, it can be ignored and $\Theta_{n}$ is simplified to:

$$
\Theta_{n}=\left(\omega_{n}+\omega_{n-1}+2 \omega_{O}\right) T+\Delta \varphi_{n},
$$

To eliminate the effect of the modulated frequency and the frequency offset on $\Theta_{n}$, another differential detection is used. Let us define $d_{n}$ as follows.

$$
\begin{aligned}
d_{n} & =\mathscr{X}_{n}\left(\Delta \hat{\omega}_{n}\right) \mathscr{X}_{n-1}^{*}\left(\Delta \hat{\omega}_{n-1}\right) \\
& =E_{S}^{2} \exp \left[j\left(\Theta_{n}-\Theta_{n-1}\right)\right]
\end{aligned}
$$

The phase component of $d_{n}$ can be written as follows.

$$
\Theta_{n}-\Theta_{n-1}=\left(\Delta \omega_{n}+\Delta \omega_{n-1}\right) T+\Delta^{2} \varphi_{n},
$$

where $\Delta \omega_{n-1}=\omega_{n-1}-\omega_{n-2}$ and $\Delta^{2} \varphi_{n}=\Delta \varphi_{n}-\Delta \varphi_{n-1}$. As can be seen in (6.15) the phase component has two terms. The first one, $\left(\Delta \omega_{n}+\Delta \omega_{n-1}\right) T$, can be ignored as it equals an integer multiple of $2 \pi$ (see the explanation before (6.13)). The second term is the double difference of the signal phase over three consecutive symbols (the same difference as used in DDPSK) and merely depends on the transmitted data using MPSK. According to (6.1), $\Delta^{2} \varphi_{n}=\alpha_{n}$ and thus:

$$
\hat{\alpha_{n}}=\arg \left(d_{n}\right)
$$

which, in the presence of noise, can be estimated using the following familiar decision rule for MPSK [94].

$$
\hat{\alpha_{n}}=\underset{\alpha_{i} \in A}{\arg \max } \operatorname{Re}\left\{d_{n} \times \exp \left(-j \alpha_{i}\right)\right\},
$$

where $A=\left\{\frac{2 \pi i}{M} \mid i=0, \ldots, M-1\right\}$ is the set of possible symbol phases for an MPSK modulation.

Now, the block diagram in Figure 6.1b can be explained based on (6.5)-(6.17). The received signal passes through an autocorrelation stage to achieve $X_{k, n}$ as in (6.6). Afterwards, the signal passes through $2 L-1$ different branches each of which including a frequency shift equal to one of the frequencies in $\Omega$ (see (6.11)) proceeding by an integrate and dump block (shown as summations). Each branch in this filter bank of Figure 6.1b is actually generating a DFT bin.

After the filter bank the upper branch calculates the magnitude of the filter output (DFT magnitude) and the max block determines the $\Delta \hat{\omega}_{n}$ based on (6.11). The result of the $\max$ block is used to select the proper path using a multiplexer (MUX) block in the lower branch. This selected output of the filter bank goes to the next stage of the differential decoding to achieve $d_{n}$ as in (6.14). Subsequently, another max block determines the MPSK symbol $\hat{\alpha}_{n}$ according 


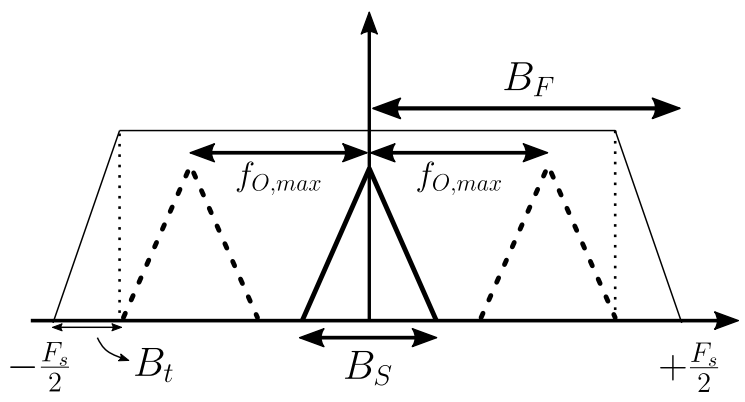

Figure 6.2: The relation between signal bandwidth $\left(B_{S}\right)$, sampling frequency $\left(F_{s}\right)$, filter bandwidth $\left(B_{F}\right)$ and maximum tolerable frequency offset $\left(f_{O, \max }\right)$

to (6.17). Finally, the detected symbols using phase and frequency are demapped to achieve the initial bits transmitted using MPSK and LFSK modulations.

It is worth mentioning that the branches in the filter bank add much less complexity compared to the DFT. The middle branch corresponding to $\Delta \omega_{n}=0$ includes only additions. Furthermore, the multiplication factors for the frequency shift in the lower half of the branches in Figure $6.1 \mathrm{~b}$, are complex conjugates of the upper half. This means that the values of the multiplications in the upper half can be reused for the lower one as well. Thus, for LFSK only $L-1$ branches include complex multiplications.

\subsubsection{Tolerable Frequency Offset}

As shown above, the effect of frequency offset on the frequency and the phase of the received signal is removed from corresponding decision variables (see (6.6) and (6.15), respectively). So in principle, the derived demodulator performs independent of the frequency offset. The main parameter which determines the frequency offset tolerance is the bandwidth of the low-pass filter prior to the demodulator (see Figure 6.1b).

Similar to what discussed in Chapter 3, a filter with bandwidth equal to $B_{F}$ as shown in Figure 6.2 is assumed. The sampling frequency (for complex samples) is set to $F_{s}=2 B_{F}$. $B_{t}$ represents the transition band of the filter which is not zero for practical filters. The signal bandwidth (null-to-null) and the maximum tolerable frequency offset are shown by $B_{S}$ and $\pm f_{O, \text { max }}$, respectively.

In Chapter 3 (section 3.5.1), it was shown that, for $B_{t}=0$, the range of the maximum tolerable frequency offset normalized to the symbol rate is (see (3.26)):

$$
-\frac{N-B_{S} / R_{S}}{2} \leq \frac{f_{O}}{R_{S}} \leq+\frac{N-B_{S} / R_{S}}{2}
$$


For hybrid modulation, the signal bandwidth, $B_{S}$, is mainly determined by the bandwidth of the FSK modulation [34]. For the proposed modulator/demodulator, the bandwidth of LFSK can be approximated (null-to-null) as follows [103] (notice that a rectangular pulse is used).

$$
B_{S}=(L-1) f_{\text {sep }}+2 R_{S}
$$

where $f_{\text {sep }}$ and $R_{S}$ are the frequency separation of the FSK modulation and the symbol rate, respectively. In the proposed system, $f_{\text {sep }}=R_{S}$ which simplifies the signal bandwidth approximation to $B_{S}=(L+1) R_{S}$. Consequently, the range of the tolerable frequency offset normalized to the symbol rate is:

$$
-\frac{N-L-1}{2} \leq \frac{f_{O}}{R_{S}} \leq+\frac{N-L-1}{2}
$$

For any $L$, the frequency offset tolerance of the proposed demodulator only depends on $N$ (which is determined by the filter bandwidth) and can be modified by increasing the bandwidth of the filter prior to the demodulator as well as the sampling frequency.

\subsection{The Proposed Demodulator in a Time-Varying Chan- NEL}

One motivation for hybrid modulation is a higher power efficiency compared to higher order PSK. On the other hand, the offset tolerant demodulator targets UNB applications which are vulnerable to temporal fading due to their long symbol period. This raises the question whether using the hybrid method with the proposed demodulator instead of higher orders of PSK has benefits when a time-varying channel is considered. To answer this question, the performance of the hybrid demodulator is analyzed in a time-varying fading channel.

The improvement of hybrid modulation in an AWGN channel and a flat fading and time-invariant Rayleigh channel has been analytically shown in literature $[16,34,35,57]$. However, to the best of our knowledge the performance of hybrid modulation in a time-varying channel has not been investigated. A timevarying channel also called frequency dispersive channel, is encountered when either the transceivers move or transceivers are fixed but the surrounding objects (scatterers) move. It implies that the received signal has a randomly varying phase and amplitude. The time varying phase is translated to a random frequency modulation which distorts the signal and leads to an error even for $S N R=\infty$ [71]. This irreducible error is called, error floor, in this work.

In [36], the performance of simple receivers for FSK and PSK is considered in a time-varying channel for low data rate applications. It is shown that, in contrast with DPSK, non-coherent FSK does not suffer from an error floor in time-varying channels. This property makes non-coherent FSK an attractive 
option for a time-varying channel. Nevertheless, the demodulators in [36] cannot tolerate frequency offset and need carrier frequency synchronization. To tolerate frequency offset, an autocorrelation structure is used in our proposed demodulator. This makes it impossible to directly extend the results of [36] to the proposed design.

In the proposed demodulator, for differential detection of FSK, a signal is multiplied with the conjugate of its delayed version. As a consequence of this stage (as shown later), a time-varying fading channel causes an error floor in the proposed method for FSK detection. In the sequel, it is shown that the proposed offset tolerant FSK demodulator can still achieve a lower error floor compared to Double Differential PSK (DDPSK) which is an offset tolerant demodulator for PSK. As a result, the better performance of a hybrid solution over higher order PSK also holds when the error floors of a hybrid method (with our proposed demodulator) and higher order DDPSK in a time-varying channels are considered.

A discrete time signal model is used for the mathematical formulation. Due to stages of differential detection it is very difficult (if not impossible) to achieve a closed form expression for the error probability in a time-varying channel. In this section, we focus on deriving an approximate but illustrative model to show the lower error floor that can be achieved using the utilized differential FSK demodulation compared to double differential PSK demodulator. For this purpose the error floors for Double Differential Binary PSK (DDBPSK) and Differential Binary FSK (DBFSK) are derived and compared.

According to the previous section, at the output of the first stage of autocorrelation (where the samples $X_{k, n}$ are generated in Figure 6.1b) for a Binary FSK modulation, $\Delta \omega_{n}$ for two consecutive symbols is either 0 or $\pm \omega_{\text {sep }} . F_{s}=4 R_{b}$ is considered in our calculations which means there are four samples per symbol $(N=4)$. For ease of notation, we consider the received signal for two consecutive symbols with a single indexing (instead of double indexing as in (6.5)) as follows.

$$
y_{k}=h_{k} r_{k}, k=1, . ., 8,
$$

where $h_{k}$ is the $k^{t h}$ sample of the complex channel gain. The $y_{k}$ samples belong to the $(n-1)^{t h}$ symbol when $1 \leq k \leq 4$ and represent samples of the $n^{\text {th }}$ symbol when $5 \leq k \leq 8$.

To model the time-varying channel, a first order autoregressive model, AR(1), similar to what is suggested in $[10,36]$ is employed. Therefore, $h_{k}$ can be written in terms of its previous sample $\left(b_{k-1}\right)$ :

$$
h_{k}=a h_{k-1}+\sqrt{1-a^{2}} \eta_{k},
$$

where $\eta_{k}$ is a circularly symmetric Gaussian random variable $\mathscr{C} \mathscr{N}\left(0, \sigma_{b}^{2}\right)$ and $a$ is the normalized autocorrelation of the channel, $R(\tau)$, for $\tau=T / 4$ (which is the sampling period) in our formulation. 
Let $H=\left[b_{1}, h_{2}, \ldots, b_{8}\right]$ denote the vector including all eight samples of the channel gain over two consecutive symbols. Denoting the first element by $h_{1}=b$ and applying (6.22) recursively, element $h_{m}$ of $H$ (for $\left.m=2,3, . ., 8\right)$ is obtained in terms of $h$.

$$
H(m)=h_{m}=a^{m-1} b+\sum_{i=1}^{m-1} a^{i-1} \sqrt{1-a^{2}} \eta_{m-i},
$$

The output of the first stage of the demodulator can be calculated as follows $(k=1, \ldots, 4)$ :

$$
Y_{k}=y_{k+4} y_{k}^{*}=b_{k+4} r_{k+4} b_{k}^{*} r_{k}^{*}
$$

Substituting $h_{k}$ from (6.23) into (6.24) and ignoring terms including $\eta_{i} \eta_{j}$, we achieve the following equations for $Y_{k}, k=1, . ., 4$ :

$$
\begin{aligned}
Y_{k}= & a^{2(k+1)}|h|^{2} X_{k}+a^{k+3} \sum_{i=1}^{k-1} a^{i-1} \sqrt{1-a^{2}} b \eta_{k-i}^{*} X_{k} \\
& +a^{k-1} \sum_{i=1}^{k+3} a^{i-1} \sqrt{1-a^{2}} b^{*} \eta_{k+4-i} X_{k}
\end{aligned}
$$

where $X_{k}=r_{k+4} r_{k}^{*}$ and $k=1, \ldots, 4$.

From (6.25) it can be seen that $Y_{k}$ includes random terms in addition to the signal component which is a consequence of random variables $\eta_{i}$. Hereafter, for error floor calculations, the random terms including $\eta_{i}$ are called distortion and the ratio of signal power to the power of these random terms is referred to as $S D R$ (Signal to Distortion Ratio).

Considering Binary FSK in Figure 6.1b, $Y_{k}$ passes through three branches of filtering to achieve $F_{-1}, F_{0}$ and $F_{+1}$ which are filter bank outputs corresponding to $-\omega_{\text {sep }}, 0$ and $+\omega_{\text {sep }}$, respectively. Due to the $X_{k}$ component in all distortion terms and the correlation between random terms in samples of $Y_{k}$ (resulting from the recursive nature of the channel model), the distortion power is not the same at the output of all filters.

The distortion caused by a time-varying channel manifests itself as a random variable with a non-uniform power spectral density. The main part of the distortion power is concentrated in the filter matched to the signal frequency $\left(\mathscr{X}_{n}\left(\Delta \hat{\omega}_{n}\right)\right)$ and the amount of distortion power in the adjacent filters is much smaller. In case the signal is at e.g. $F_{+1}$, the distortion power in the non-adjacent filter $\left(F_{-1}\right)$ is even negligible.

Based on this distortion, the error probability for BFSK is calculated. Since the Gaussian (receiver) noise is assumed to be zero, this error probability provides us with an error floor. The frequency component of $X_{k}$ in (6.25) is either $\Delta \omega_{n}=0$ 
or $\Delta \omega_{n}= \pm \omega_{\text {sep }}$. When $\Delta \omega_{n}=0$ the error occurs if either $\left|F_{+1}\right|>\left|F_{0}\right|$ or $\left|F_{-1}\right|>\left|F_{0}\right|$. On the other hand, when $\Delta \omega_{n}$ is $+\omega_{\text {sep }}$ or $-\omega_{\text {sep }}$, the error happens if $\left|F_{0}\right|>\left|F_{+1}\right|$ or $\left|F_{0}\right|>\left|F_{-1}\right|$, respectively. $\Delta \omega_{n}$ is zero if two consecutive BFSK symbols are the same; which has a probability of $1 / 2$. If symbols are different, $\Delta \omega_{n}$ will be either $+\omega_{\text {sep }}$ or $-\omega_{\text {sep }}$ with a probability of $1 / 4$ for each. Therefore, the probability of error is as follows:

$$
\begin{aligned}
P_{e, B F S K}= & 0.5\left[P\left(\left|F_{+1}\right|>\left|F_{0}\right| \mid \Delta \omega_{n}=0\right)\right. \\
& \left.+P\left(\left|F_{-1}\right|>\left|F_{0}\right| \mid \Delta \omega_{n}=0\right)\right] \\
& +0.25 P\left(\left|F_{0}\right|>\left|F_{-1}\right| \mid \Delta \omega_{n}=-\omega_{d}\right) \\
& +0.25 P\left(\left|F_{0}\right|>\left|F_{+1}\right| \mid \Delta \omega_{n}=+\omega_{d}\right)
\end{aligned}
$$

Each error probability in (6.26) is similar to the error probability for noncoherent BFSK when a matched filter (correlation) based demodulator is used [45]. However, there is a significant difference that must be taken into account. The conventional error probability function of non-coherent BFSK (based on matched filters [45]) is derived based on the assumption of equal noise power density in all filters which is not the case here. This problem can be solved by calculating the noise power in the matched filter and the adjacent filter and recalculate the error probabilities using the modified noise power for each filter. Each individual term in (6.26) is in the form of $P\left(\left|F_{x}\right|>\left|F_{y}\right|\right)$ (where $F_{x}$ and $F_{y}$ are adjacent filters) conditioned on the fact that the signal is in $F_{y}$. Therefore, the derivation of all the probabilities in (6.26) are the same and the mathematical derivation is continued for $P_{e}=P\left(\left|F_{+1}\right|>\left|F_{0}\right| \mid \Delta \omega_{n}=0\right)$.

According to the demodulator structure in Figure 6.1b, and in case $\Delta \omega_{n}=0, F_{0}$ and $F_{+1}$ can be written as follows.

$$
\begin{aligned}
& F_{0}=\sum_{k=1}^{4} Y_{k}=\sqrt{P_{S}} e^{-j \Delta \varphi_{n}}+\sqrt{P_{N, 0}} \eta_{0}^{T} \\
& F_{+1}=\sum_{k=1}^{4} Y_{k} e^{-j\left(\Delta \omega_{s e p} k T_{s}\right)} \approx \sqrt{P_{N,+1}} \eta_{1}^{T}
\end{aligned}
$$

where $\eta_{0}^{T}$ and $\eta_{1}^{T}$ are $\mathscr{C} \mathscr{N}(0,1), e^{-j \Delta \varphi_{n}}$ denotes the modulated phase and $E_{S} / N$ is considered to be one without loss of generality. $P_{S}, P_{N, 0}$ and $P_{N,+1}$ are as follows.

$$
\begin{array}{r}
P_{S}=S(a)|b|^{4} \\
P_{N, 0}=\Upsilon_{0}(a)|b|^{2} \sigma_{b}^{2}\left(1-a^{2}\right) \\
P_{N,+1}=\Upsilon_{+1}(a)|b|^{2} \sigma_{b}^{2}\left(1-a^{2}\right)
\end{array}
$$


where $S(a), \Upsilon_{0}(a)$ and $\Upsilon_{+1}(a)$ are functions of $a$ which is $J_{0}\left(2 \pi v_{\max } T / 4\right)$ for a Rayleigh fading channel with a Jakes spectrum and a maximum Doppler shift of $\cup_{\max }$. To simplify notations, in the following, $S(a), \Upsilon_{0}(a)$ and $\Upsilon_{+1}(a)$ are shown by $S, \Upsilon_{0}$ and $\Upsilon_{+1}$, respectively. The process of deriving (6.27)-(6.31) and detailed expressions are presented in Appendix C.

$P\left(\left|F_{+1}\right|>\left|F_{0}\right| \mid \Delta \omega_{n}=0\right)$ (denoted by $P_{e}$ in the following) can be written as follows [45].

$$
P_{e}=\int_{0}^{+\infty} P\left(\left|F_{+1}\right|>\left|F_{0}\right||| F_{0} \mid\right) f_{\left|F_{0}\right|}\left(\left|F_{0}\right|\right) d\left|F_{0}\right|
$$

where $f_{\left|F_{0}\right|}\left(\left|F_{0}\right|\right)$ is the probability density function (pdf) of $\left|F_{0}\right|$ when $\Delta \omega_{n}=0$. Notice that the signal component of $F_{+1}$ is negligible and is ignored in (6.28). Since $\eta_{1}^{T} \sim \mathscr{C} \mathscr{N}(0,1)$, it is concluded that $F_{+1} \sim \mathscr{C} \mathscr{N}\left(0, P_{N,+1}\right)$. Hence, $\left|F_{+1}\right|$ has a Rayleigh distribution with $\sigma^{2}=P_{N,+1} / 2$. Thus, we have:

$$
P\left(\left|F_{+1}\right|>\left|F_{0}\right||| F_{0} \mid\right)=\int_{\left|F_{0}\right|}^{\infty} f_{F_{1}}(x) d x=\exp \left(\frac{-\left|F_{0}\right|^{2}}{P_{n,+1}}\right)
$$

where $f_{F_{1}}(x)$ is the pdf of $\left|F_{+1}\right|$ (when $\Delta \omega_{n}=0$ ) as follows.

$$
f_{F_{1}}(x)=\frac{2 x}{P_{N,+1}} \exp \left(-\frac{x^{2}}{P_{N,+1}}\right), x \geq 0
$$

Substituting (6.33) into (6.32), using values in (6.29)-(6.31) and following a procedure similar to calculations in [45] (equations (7.231)-(7.243)) for non-coherent orthogonal modulations, the following equation for the error probability can be obtained.

$$
P_{e}=\frac{P_{N,+1}}{P_{N,+1}+P_{N, 0}} \exp \left(-\frac{P_{S}}{P_{N,+1}+P_{N, 0}}\right)
$$

Substituting $P_{S}, P_{N, 0}$ and $P_{N,+1}$ from (6.29)-(6.31) we have:

$$
P_{e}=\frac{\Upsilon_{+1}}{\Upsilon_{+1}+\Upsilon_{0}} \exp \left(-\frac{S|b|^{2}}{\left(\Upsilon_{+1}+\Upsilon_{0}\right)\left(1-a^{2}\right) \sigma_{b}^{2}}\right)
$$

Now, we have the error probability which depends on $|b|^{2}$. To achieve the average error probability we need to integrate it over $|h|^{2}$. For each set of three symbols, $h$ is a Gaussian complex random variable $h \sim \mathscr{C} \mathscr{N}\left(0, \sigma_{b}^{2}\right)$ according to the definition of a Rayleigh channel. Thus, it is concluded that $|h| \sim \operatorname{Rayleigh}\left(\sigma_{b}^{2} / 2\right)$ 
and $|h|^{2} \sim \operatorname{Exp}\left(1 / \sigma_{h}^{2}\right)$ where Exp is the exponential distribution with the following pdf:

$$
f(x)=\frac{1}{\sigma_{b}^{2}} \exp \left(-\frac{x}{\sigma_{b}^{2}}\right), x \geq 0
$$

Replacing $|h|^{2}$ with $x$ in (6.36) and using (6.37), the average error probability $\overline{P_{e}}$ is achieved as follows [97].

$$
\begin{aligned}
\bar{P}_{e} & =\int_{0}^{\infty} P_{e}(x) f(x) d x \\
& =\frac{\left(1-a^{2}\right) \Upsilon_{+1}}{\left(1-a^{2}\right)\left(\Upsilon_{0}+\Upsilon_{+1}\right)+S}
\end{aligned}
$$

As mentioned earlier, each individual probability in (6.26) is derived using the same process and their values are the same. Thus, the error floor $\left(E F_{D B F S K}\right)$ is obtained by replacing each probability term in (6.26) with $\overline{P_{e}}$ from (6.38).

$$
E F_{D B F S K}=\frac{3}{2} \frac{\left(1-a^{2}\right) \Upsilon_{+1}}{\left(1-a^{2}\right)\left(\Upsilon_{0}+\Upsilon_{+1}\right)+S}
$$

Now, we need to calculate the error floor for DDBPSK. In a DDBPSK demodulator, the signal after the first stage is equal to $F_{0}$ which is used as the input of the second stage. According to (6.27), $F_{0}$ has a signal component and a Gaussian noise (distortion) component. It can be seen as a signal with an SNR equal to $P_{S} / P_{N, 0}$. Since for DDBPSK, the second stage is simply differential detection of antipodal symbols, the error probability is derived as follows [97].

$$
P_{e, D P S K}=\frac{1}{2} \frac{1}{1+\overline{S N R}},
$$

To calculate the error floor, it is assumed that there is no receiver noise (AWGN). As mentioned earlier, the Gaussian random variables in the above equations are a consequence of time-variation of the channel. Thus, instead of SNR in (6.40), we may use the term, SDR (Signal to Distortion Ration), which is as follows.

$$
S D R=\frac{P_{S}}{P_{N, 0}}=\frac{S|h|^{2}}{\Upsilon_{0}\left(1-a^{2}\right) \sigma_{b}^{2}}
$$

Considering the pdf of $|h|^{2}$ as in (6.37) and integrating over $|b|^{2}$, the average SDR is: 


$$
\overline{S D R}=\frac{S}{\Upsilon_{0}\left(1-a^{2}\right)}
$$

Substituting (6.42) into (6.40), the error floor for DDBPSK is achieved as follows.

$$
E F_{D D B P S K}=\frac{\Upsilon_{0}\left(1-a^{2}\right)}{2 \Upsilon_{0}\left(1-a^{2}\right)+2 S},
$$

Figure 6.3 demonstrates the error floor for different values of the maximum Doppler shift in a Rayleigh fading channel with a Jakes Doppler spectrum. Solid lines show values obtained from (6.39) and (6.43) while dashed lines are simulation results. It can be seen that the theoretical and simulation results are in agreement while there is a slight difference for the higher values of $v_{\max }$. In our approximations the multiplication of noise terms is ignored. This assumption does not hold for large values of Doppler shift which increases the variance of the random component in (6.22). Thus, as $u_{\max }$ increases, the theory slightly deviates from simulations. Figure 6.3 demonstrates that the error floor achieved by DBFSK using the proposed demodulator is lower than the one that can be obtained by the conventional DDBPSK. Increasing the modulation order of DDPSK is equivalent to using a higher order DPSK for which the input signal is modeled as (6.27). This leads to even higher error. As a result, if modulation order is increased by combining DLFSK and DDPSK, the error floor is expected to be lower compared to the error floor achieved when increasing the order of DDPSK.

\subsection{System Design for Time Diversity}

In UNB communications, the proposed offset tolerant demodulator can be utilized to increase the bit-rate and employ channel coding and interleaving so that the time diversity which is provided by a time-varying channel is exploited.

In a general case of using time diversity, a system similar to Figure 6.4a can be employed. First, information bits are sent to the channel encoder. Then, an interleaver is used to make sure that the coded bits belonging to one codeword are distributed in time and experience different channel fading conditions. In the next step, the sequence of bits is split into two sets; $B_{P S K}$ and $B_{F S K}$. The former set is modulated using MPSK and the latter is sent using LFSK. The bits in $B_{P S K}$ and $B_{F S K}$ are modulated using the hybrid modulator and transmitted. After passing through the channel and adding AWGN noise, the samples of the signal enter the demodulator. The output of the demodulator is fed to the Mix and De-Interleave block which puts the two sets of the detected bits together and deinterleaves them. In the last step, the channel decoder uses the detected and deinterleaved bits to extract information bits. In the block diagram of Figure 6.4a any channel coding can be used. 


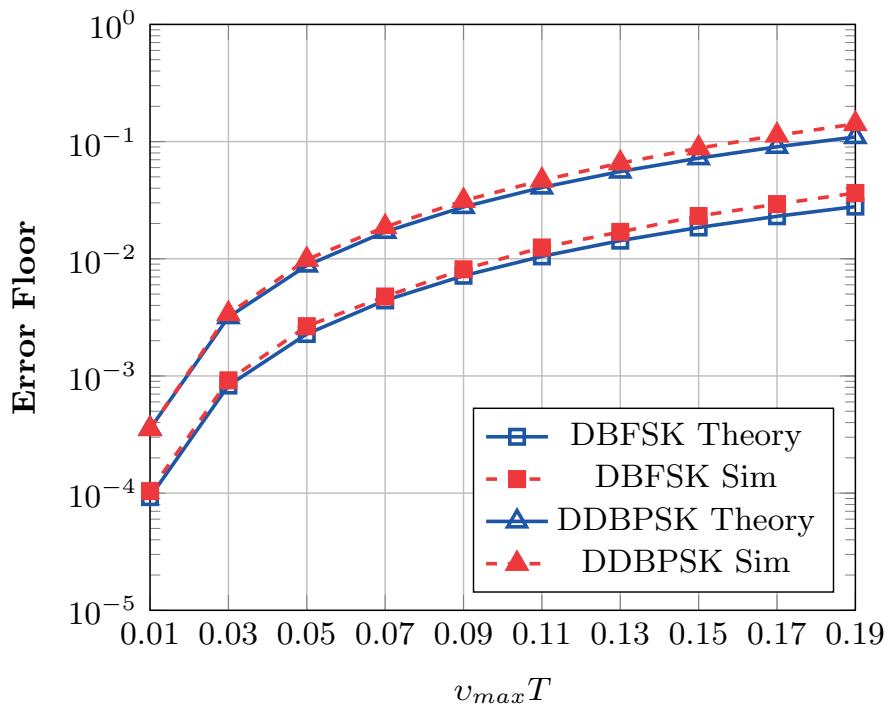

Figure 6.3: Simulated and theoretical error floor versus normalized maximum Doppler shift in a Rayleigh fading channel with Jakes Doppler spectrum

In this work we focus on the simplest form of channel coding which is repetition coding. Assuming a packet of information bits, the increased bit-rate is used to re-transmit the information packet several times during the same packet-time that is needed for BFSK (2FSK) or BPSK (2PSK) modulation to transmit the same set of information bits. Figure $6.4 \mathrm{~b}$ and Figure $6.4 \mathrm{c}$ show the scheme used in the dashed box A of Figure 6.4a. Notice that hybrid LFSK/MPSK modulation makes it possible to transmit $\log _{2}(M)+\log _{2}(L)$ replicas of the information bits in the same packet time. Another way of increasing the bit-rate is using higher order DDPSK (DDMPSK). In the following sections, the BER performance of hybrid modulation is compared to different orders of DDMPSK. Using only DDMPSK the number of replicas is $\log _{2}(M)$ (see Figure 6.4b). Figure 6.4c visualizes how the bits in each replica of information packet are split between $B_{F S K}$ and $B_{P S K}$.

In case of temporal fading, the replicas transmitted at different time instances experience different channel fading and making decisions considering these instances will improve the BER performance. In the system shown in Figure 6.4a, in a general case, the bits of a codeword (which are interleaved and split in time) are de-interleaved after demodulation and detection. For a repetition coding scheme, an option for the decoder is majority voting. For each information bit, voting over various detected replicas determines the final value. However, in this way all replicas have an equal effect on the final decision regardless of their corresponding instantaneous SNR. Including the effect of the instantaneous SNR value decreases the probability of error in the final decision. Assume that the 

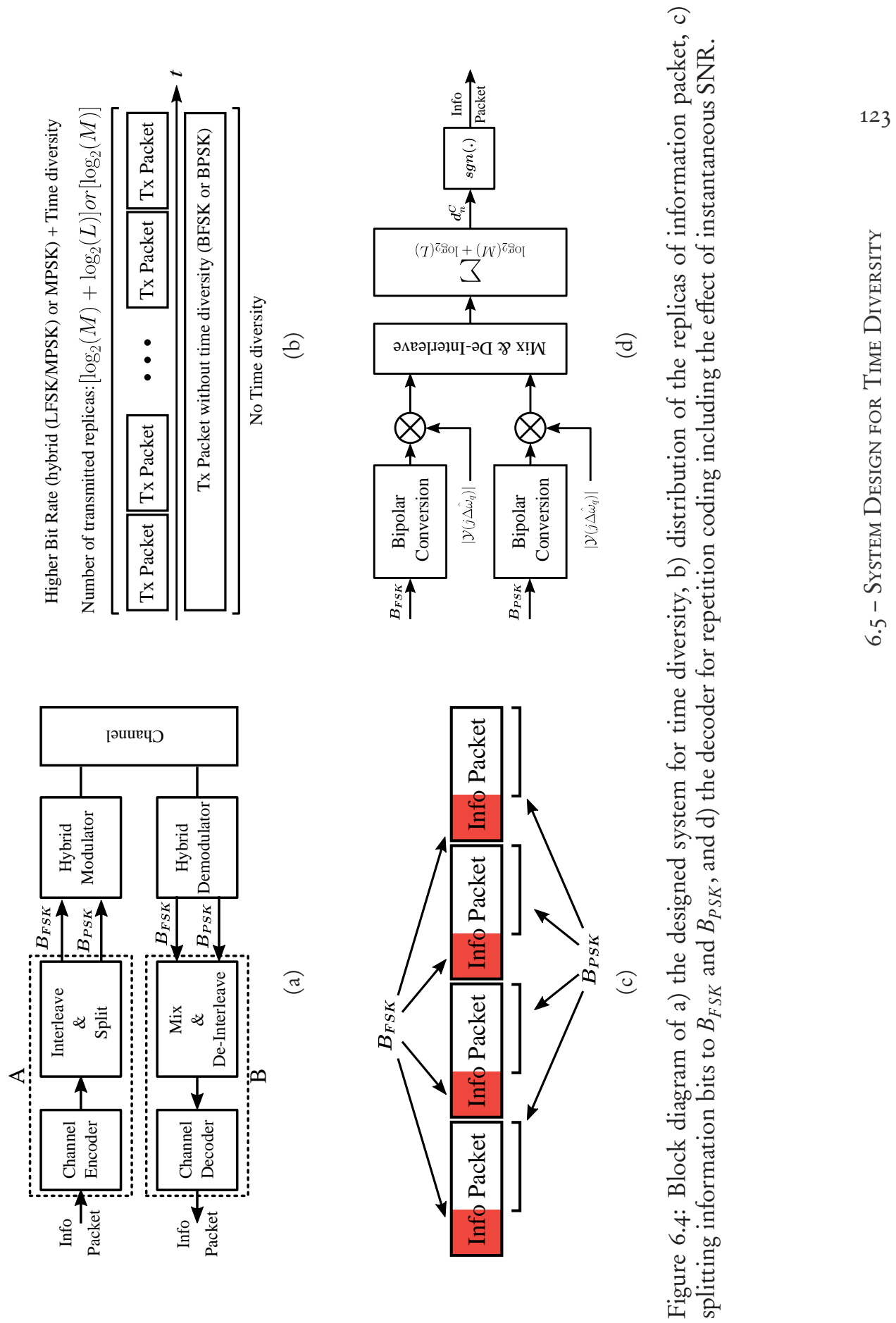
fading channel coefficient is equal to $h_{n}$ for the $n^{\text {th }}$ symbol. Then, the received signal $y_{n}$ is as follows:

$$
y_{n}=h_{n} r_{n}
$$

where $r_{n}$ is the same as in (6.5). The instantaneous received signal power (which determines the instantaneous SNR) is proportional to $\left|h_{n}\right|^{2}$. Using calculations similar to (6.6) and (6.12), an equivalent to $\mathscr{X}_{n}\left(\Delta \hat{\omega}_{n}\right)$ in presence of a fading channel, denoted by $\mathscr{Y}_{n}\left(\Delta \hat{\omega}_{n}\right)$, can be derived as follows.

$$
\begin{aligned}
\mathscr{Y}_{n}\left(\Delta \hat{\omega}_{n}\right) & =\sum_{k=0}^{N-1} Y_{k, n} \exp \left[-j \Delta \hat{\omega}_{n} k T_{s}\right] \\
& =E_{S} / N \exp \left[j \Theta_{n}\right] \sum_{k=0}^{N-1} h_{k, n} b_{k, n-1}^{*}
\end{aligned}
$$

where $Y_{k, n}$ is the $k^{t h}$ sample of $Y_{n}=y_{n} y_{n-1}^{*}$. In the proposed demodulator, the decision for FSK is made based on finding the maximum of $\left|\mathscr{Y}_{n}\left(j \omega_{i}\right)\right|$. The output of the filter chosen based on this maximum, $\mathscr{Y}_{n}\left(\Delta \hat{\omega}_{n}\right)$, is sent to the next stage of differential decoder to detect DDMPSK. Assuming that the fading coefficients are constant during three symbols $(n, n-1$ and $n-2)$ and equal to $h_{n},\left|\mathscr{Y}_{n}\left(\Delta \hat{\omega}_{n}\right)\right|$ is proportional to the received signal power $\left(E_{S} / N\right)\left|h_{n}\right|^{2}$ during these three symbols.

To determine the transmitted bit, the detected replicas of the $i^{\text {th }}$ information bit are weighted and added as follows (which resembles to maximum ratio combining).

$$
d_{i}^{C}=\sum_{q=1}^{Q}\left|\mathscr{Y}_{q}\left(j \Delta \hat{\omega}_{q}\right)\right| b_{q}
$$

where $b_{q} \in\{-1,+1\}$ is the bipolar conversion of the detected bits. $q$ is the index of the symbol with which the $q^{t h}$ replica of the $i^{t h}$ information bit is transmitted. $Q$ is the number of replicas of the $i^{\text {th }}$ information bit which are transmitted, $Q=\log _{2}(L)+\log _{2}(M)$. Since the bipolar coded bits are used in obtaining $d_{i}^{C}$, the information bit is determined by the sign of $d_{i}^{C}$.

To summarize, for a replica of an information bit experiencing channel coefficient $h_{n}$ during its transmission, the influence of $\left|h_{n}\right|^{2}$ is included using a weighted voting. The detailed block diagram of the dashed box B in Figure 6.4a based on (6.46) is demonstrated in Figure 6.4d. For each symbol, the detected bits in LFSK and MPSK are sent to the mixing and decoding block with their corresponding, $\left|\mathscr{Y}_{n}\left(\Delta \hat{\omega}_{n}\right)\right|$. The detected bits are converted to bipolar format. Each $b_{q}$ is weighted according to (6.46) and sent to the deinterleaver. Since 
repetition coding is used, the de-interleaved sequence includes $\log _{2}(M)+\log _{2}(L)$ replicas of each information bit in a row. Thus, $d_{i}^{C}$ is obtained by adding these values in the summation block. In the final step, a signum function $(\operatorname{sgn}()$. determines the information bit.

\subsection{BER Performance in AWGN Channel}

The main motivation for the proposed offset-tolerant demodulator is achieving a better BER performance compared to higher order MPSK modulation in ultranarrowband applications. The proposed hybrid demodulator is compared with an offset tolerant demodulator for MPSK (DDMPSK). For MPSK modulation, the Double Differential MPSK (DDMPSK) introduced in [94] is utilized. The DDMPSK modulator is the same as the upper branch of the modulator in Figure 6.1a. The DDMPSK demodulator is composed of two stages of differential decoding similar to the first stage of the demodulator in Figure 6.1b.

For an AWGN channel, the information bits are split and sent to the hybrid modulator without any repetition or interleaving. After the signal passes through an AWGN channel, it is demodulated and the transmitted data is detected using a hybrid demodulator.

To simplify comparison, the combinations of hybrid modulations are divided into two sets. One including 2PSK and 4PSK (shown by $2 \mathrm{P}$ and $2 \mathrm{~F}$, respectively) and their combinations with 2FSK and 4FSK (Figure 6.5a); whereas, the other set includes 8PSK and 16PSK (shown by $8 \mathrm{P}$ and 16P, respectively) and their combinations with 2FSK and 4FSK (Figure 6.5b). In AWGN channel simulations, each transmitted bit is an information bit with $E_{b}^{\operatorname{Inf}}$ energy (notice that no repetition is used in AWGN channel). Moreover, each symbol in the hybrid modulation represents $\log _{2}(L)+\log _{2}(M)$ bits; thus, the energy transmitted per symbol is $E_{S}=\left(\log _{2}(L)+\log _{2}(M)\right) E_{b}^{I n f}$. In the following a combination of LFSK and MPSK is shown by $L F M P$, while MPSK or LFSK are denoted by $M P$ and $L F$, respectively. $L F x P$ denotes a set of combinations of LFSK with MPSK for all $M$.

As can be seen in Figure 6.5, increasing the FSK order improves the BER performance. It is also much more power efficient compared to increasing the PSK order (red dotted curves) when a certain overall modulation order $\left(\log _{2}(L)+\right.$ $\left.\log _{2}(M)\right)$ is the target. According to Figure 6.5, using the proposed offset tolerant demodulator, hybrid modulation achieves a better performance compared to the offset tolerant demodulator for MPSK (DDMPSK). For instance, 2F4P and 8PSK both achieve the same modulation order; however, 2F4P saves almost $6 \mathrm{~dB}$ energy at BER $=0.1 \%$ compared to DD8PSK. This energy saving compared to the higher order MPSK is the same as what has been shown in $[34,52,57]$.

Substituting $N=10$ and $L=2,4$ in (3.25)-(6.20), the maximum tolerable frequency offset normalized to the symbol rate, $f_{O, \max } / R_{S}$, for $2 \mathrm{FxP}$ and $4 \mathrm{FxP}$ is 


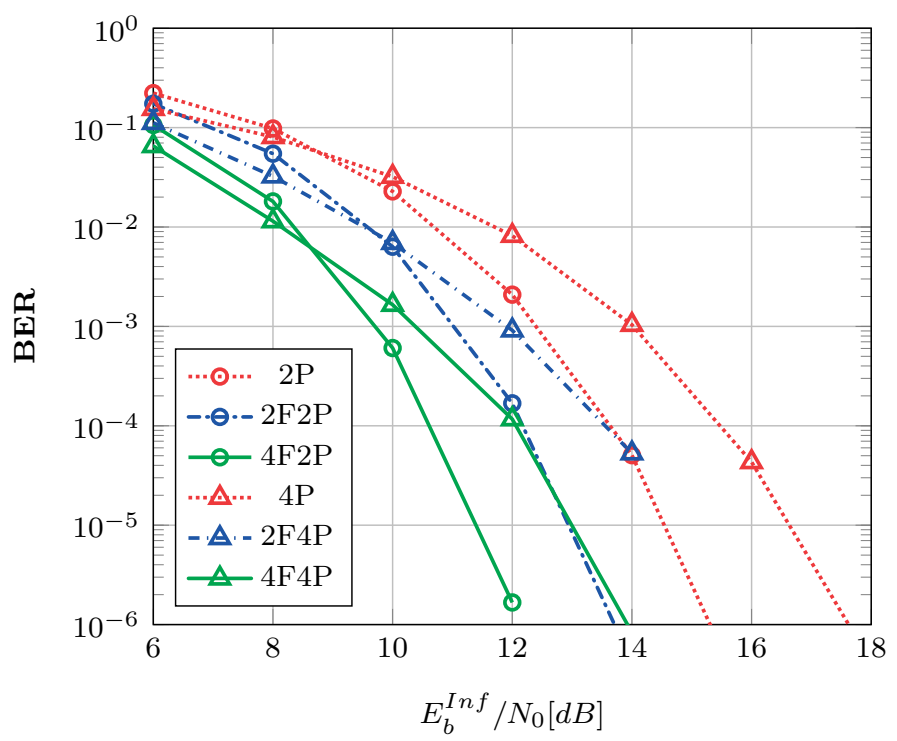

(a)

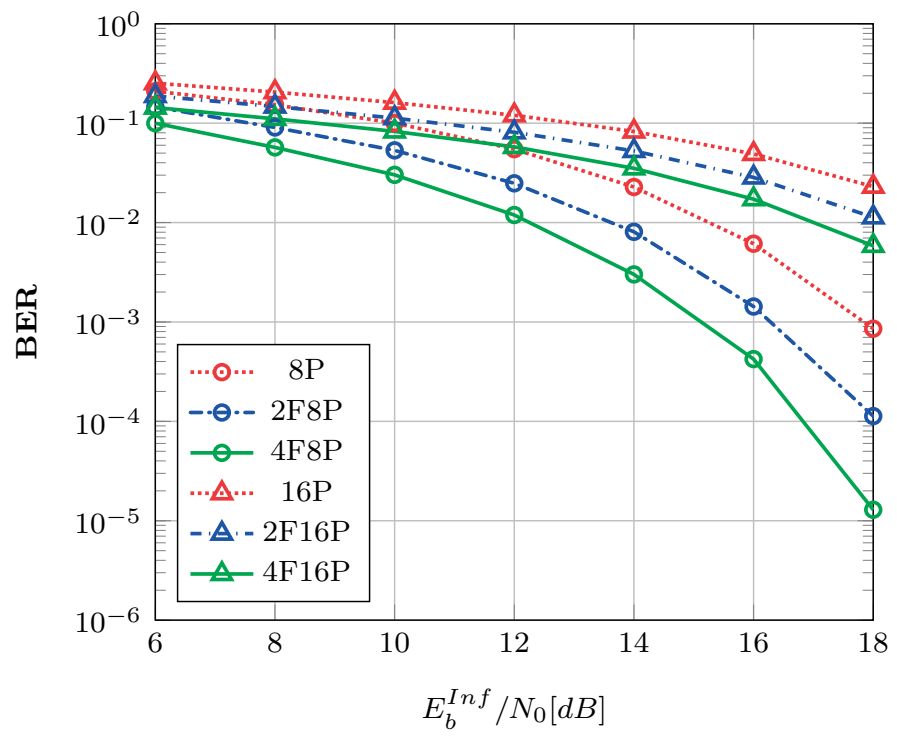

(b)

Figure 6.5: BER curves for hybrid modulation in an AWGN channel for combination of 2FSK and 4FSK with a) 2PSK and 4PSK, b) 8PSK and 16PSK 


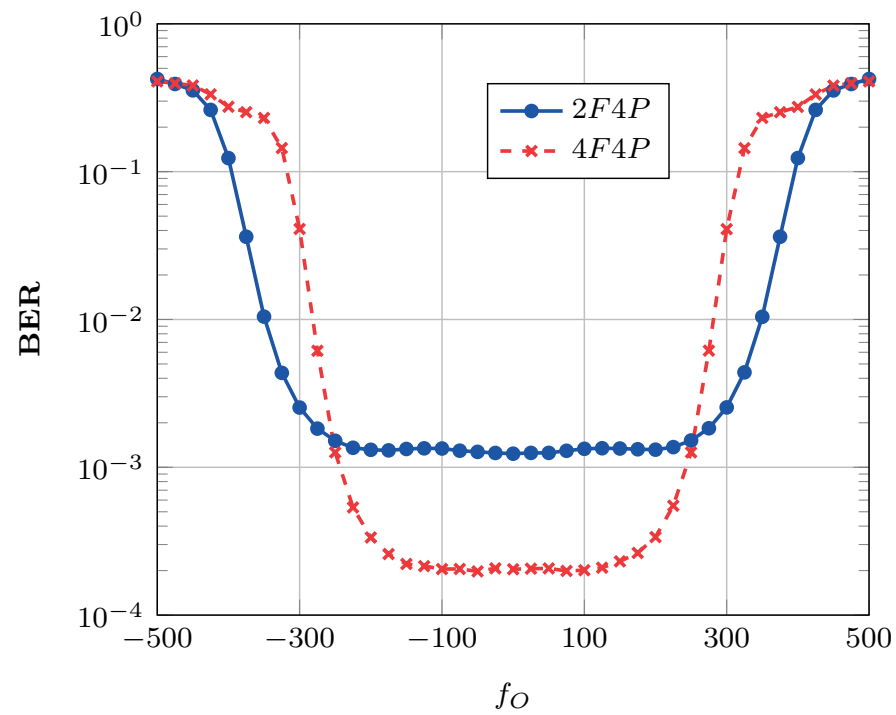

Figure 6.6: BER performance of hybrid modulation for 2F4P and 4F4P. An inverse-sinc low-pass filter (see Figure 6.1b) is used with $B_{t}=100 \mathrm{~Hz}$ and $B_{F}=1$ $\mathrm{kHz}$ while $R_{S}=100 \mathrm{Sym} . / \mathrm{s}$ and $F_{s}=1 \mathrm{kHz}$

3.5 and 2.5, respectively. The ability of the proposed demodulator to tolerate frequency offset is demonstrated in Figure 6.6 where BER values for $2 \mathrm{~F}_{4} \mathrm{P}$ and $4 \mathrm{~F} 4 \mathrm{P}$ at $E_{b} / N_{0}=12 \mathrm{~dB}$ can be seen when $-500 \mathrm{~Hz}<f_{O}<+500 \mathrm{~Hz}$. To show the effect of the filter frequency response on the offset tolerance, an inverse-sinc low-pass filter is considered with $B_{t} \approx 100 \mathrm{~Hz}$ and $B_{F}=500 \mathrm{~Hz}$. The symbol rate is $100 \mathrm{Sym} . / \mathrm{s}$ and $F_{s}=1 \mathrm{kHz}$. As can be seen the BER is constant for $\left|f_{\mathrm{O}}\right|<250 \mathrm{~Hz}$ and $f_{\mathrm{O}}<150 \mathrm{~Hz}$, in case of $2 \mathrm{~F} 4 \mathrm{P}$ and $4 \mathrm{~F} 4 \mathrm{P}$, respectively. These values are $100 \mathrm{~Hz}$ less than the expected values which is a consequence of nonzero $B_{t}$ equal to $100 \mathrm{~Hz}$. The increase in the BER for a larger frequency offset is not a consequence of the demodulator characteristics but due to lack of coverage by the filter. To solve this, a wider filter can be used and the sampling frequency must increase accordingly.

\subsection{BER Performance in a Time-Varying Channel}

To configure the system and perform comparisons, a few assumptions, criteria and definitions are introduced. This is necessary to clarify further discussions and guarantee a fair comparison. These are summarized as follows:

1. It is assumed that a constant number of information bits is required to be transmitted denoted by $T X^{\operatorname{Inf}}$. 
2. A reference symbol rate, $R_{S}^{R e f}$, is defined as a baseline. This is equal to the information bit-rate $R_{b}^{\operatorname{Inf}}$ in case of a simple 2PSK transmission.

3. A reference packet time, $T_{P}^{R e f}$, is defined. The packet time in this simplest case (2PSK) is the time needed to send $T X^{\operatorname{Inf}}$ information bits with a symbol rate equal to $R_{S}^{R e f}$. The symbol rate is equal to the bit-rate in this simplest case. The target is to keep $T_{P}^{R e f}$ constant as it determines the RF circuit power consumption which is a significant contributor to the node power consumption. As a result, transmitting more bits for redundancy just by prolonging the overall transmission time is not included in this comparison as it is considered not to be efficient.

4. A constant energy per information bit is considered as $E_{b}^{\operatorname{Inf}}$. The target is to improve performance without increasing the energy transmitted per information bit. In other words, redundant information is sent with lower energy to make sure that the overall transmitted energy per information bit remains constant. This affects the energy per transmitted symbol which is discussed further for each scenario.

5. Higher order PSK and the hybrid modulation scheme are used to increase the raw bit-rate and transmit redundant information for time diversity. Since in this work we use repetition coding, Repetition Order (RO) is defined as the ratio of the achieved raw bit-rate and $R_{S}^{R e f}$. This is, in fact, the inverse of the code rate. For instance, 2PSK has a repetition order of 1 and a combination of 2FSK and 4PSK (shown by 2F4P) provides a repetition order of 3 ( 1 from 2 FSK and 2 from 4 PSK).

In our simulations, $T X^{\operatorname{Inf}}$ equals 240 which is in the same order of short packet lengths used in UNB applications $[2,55]$. Besides, it is divisible by the repetition orders achieved using hybrid combinations and higher order MPSK which are discussed here. This makes the analysis easier and more straightforward without loss of generality. The reference symbol rate $R_{S}^{R e f}$ is equal to $100 \mathrm{Sym} . / \mathrm{s}$ which is also the same as in $[2,55]$. This makes $T_{P}^{R e f}$ equal to $2.4 \mathrm{~s}$. The rest of the scenarios and parameters are built up according to this baseline. In each application, these parameters can be set accordingly. Then, the insight provided by the following results can be used to understand the trade-offs and choose the best modulation scheme.

The Rayleigh fading (with Jakes spectrum) channel is considered as the worst case channel. A popular application for UNB communication is fixed wireless links. In these links the transmitter and the receiver nodes are static and the temporal fading is caused by movement of the scatterers (such as cars, people and foliage in the wind). Several measurement campaigns and a large amount of research has been performed to model the channel in such applications in different environments $[5,40,51,77]$ (though they do not specifically consider UNB). Elaborating on the channel models and their analysis in fixed wireless 
links is not in the scope of this thesis. Nevertheless, to illustrate the performance of the proposed method in different channel characteristics, simulation results for a more realistic channel model in fixed wireless scenarios are presented as well. According to $[28,77]$, in a fixed wireless link, the channel model is more similar to Rician channel and the Doppler shift has a higher distribution density around zero. Hence, a more realistic channel model is a Rician channel with a rounded Doppler spectrum rather than a Rayleigh channel with the Jakes spectrum of which simulation results will also be presented [28].

The error floor analysis in the previous section was performed for the worst case which is a Rayleigh channel. The BER performance for both Rayleigh and Rician channel based on simulations will be presented in the following. The $\mathrm{K}$-factor in a Rician channel depends on several parameters. In our simulations, for the Rician channel, we have considered $K=1$ which means that the power of the line-of-sight component is equal to the power summed over the multipath components. Furthermore, in the scenarios with the highest Doppler spread considered for a fixed wireless channel in [28], the maximum Doppler shift is around $2 \mathrm{~Hz}$. To show the effect of a higher maximum Doppler shift, the proposed demodulator is also evaluated for $D_{\max }=5 \mathrm{~Hz}$. In a time-invariant channel, using time-diversity does not lead to improvement as it does not add any diversity; therefore, the results for a time-invariant channel are not presented.

For MPSK, four different values of $M$ are considered $(M=2,4,8,16)$. This set of modulation orders will clearly show the intended trade-offs. For LFSK, $L=2,4$ are considered since small L-values impose lower bandwidth overhead. These values for $\mathrm{M}$ and $\mathrm{L}$ form eight different combinations for hybrid modulation which are analyzed in the simulations. The number of samples per symbol is considered to be $N=10$. Table 6.2 summarizes the basic simulation parameters.

As mentioned earlier, increasing the raw bit-rate, in an efficient way, is a motivation for hybrid modulation. For MPSK modulation, the ratio of the raw bit-rate to $R_{S}^{R e f}$, what we defined as repetition order (RO), is equal to $\log _{2}(M)$. For hybrid modulation composed of LFSK and MPSK, this repetition order equals:

$$
R O=\log _{2}(L)+\log _{2}(M)
$$

Table 6.3 demonstrates the combinations and the repetition order which can be achieved using them. In the following subsections, two different scenarios are considered for evaluating hybrid modulation in a time-varying fading channel; constant symbol rate and equal bandwidth.

\subsubsection{Constant Symbol Rate Scenario}

The symbol rate of a communication link determines the effect of a time-varying channel on the BER performance. In fact, the severity of the temporal fading depends on the maximum Doppler shift normalized to the symbol rate $\left(D_{\max } T\right)$ 
Table 6.2: Simulation parameters

\begin{tabular}{|c|c|}
\hline$T X_{\text {Inf }}$ & 240 \\
\hline$R_{S}^{\text {Ref }}$ & 100 \\
\hline$T_{P}^{\text {Ref }}[\mathrm{s}]$ & 2.4 \\
\hline Maximum Doppler shift $\left(D_{\max }\right)[\mathrm{Hz}]$ & 2,5 \\
\hline K factor for Rician channel & 1 \\
\hline$N($ Sample $/$ Symbol $)$ & 10 \\
\hline$L$ & 2,4 \\
\hline$M$ & $2,4,8,16$ \\
\hline
\end{tabular}

Table 6.3: Different combinations and the corresponding repetition order

\begin{tabular}{|c|c|c|c|}
\hline & No FSK & 2FSK & 4FSK \\
\hline No PSK & - & 1 & 2 \\
\hline 2PSK & 1 & 2 & 3 \\
\hline 4PSK & 2 & 3 & 4 \\
\hline 8PSK & 3 & 4 & 5 \\
\hline 16PSK & 4 & 5 & 6 \\
\hline
\end{tabular}

[71]. Therefore, to keep the effect of temporal fading constant for comparison, the symbol rate is maintained constant in this scenario. Since the bandwidth usage in UNB communications is not critical, it is reasonable to assume that the bandwidth increase due to using FSK modulation can be afforded. The higher repetition order achieved by hybrid modulation or higher order DDMPSK is used for adding time diversity with repetition coding using the system in Figure 6.4 .

As the symbol rate and packet time (the third criterion mentioned at the beginning of this section) are constant in this scenario, the number of symbols that are transmitted remains constant and equal to $T X^{\operatorname{Inf}}$. For any modulation in Table 6.3, the repetition order $(R O)$ shows the number of replicas that can be transmitted for each information bit. Based on the fourth criterion, the $E_{b}^{\operatorname{Inf}}$ is kept constant which requires $E_{b}^{\operatorname{Inf}}$ to be distributed over all redundant bits. As a result, energy per transmitted bit, $E_{b}$, is $E_{b}^{I n f} / R O$. Finally, each symbol includes $R O$ bits so the energy per transmitted symbol in our scenario is equal to the energy per information bit.

For a better performance, error correcting codes are usually used instead of simple repetition coding. Hence, the interesting part of the BER curve without error correction code is around $B E R=0.1 \%$. To provide an overview, the $E_{b}^{\operatorname{Inf}} / N_{0}$ 
required for $B E R=0.1 \%$ for Rayleigh and Rician channels and $D_{\text {max }}=2,5 \mathrm{~Hz}$ are shown in Figure 6.7 (the BER curves for different combinations and channel models can be found in Appendix D). The horizontal axis shows the order of PSK modulation. Each bar represents stand alone PSK (red), PSK in combination with 2FSK (blue) and PSK in combination with 4FSK (green). When the PSK order equals 1 (the leftmost bars in Figure 6.7), there is no phase modulation so these cases refer to pure FSK modulation. The bars hit the maximum of the vertical axis when the error floor is higher than $B E R=0.1 \%$ (i.e. they never reach $B E R=0.1 \%$ ). It is clearly seen that 4 PSK performs the best when combinations with FSK (blue and green bars) are considered. Furthermore, increasing FSK order improves performance for all PSK orders. It is also seen that for hybrid modulation, 4 is the best choice for PSK order. It increases diversity but degrades BER performance less significantly compared to 8PSK and 16PSK.

\subsubsection{Equal Bandwidth Scenario}

The improvement in the BER performance of hybrid modulation, compared to DDMPSK, is obtained at the expense of higher bandwidth usage. Increase in bandwidth is not prohibitive in UNB applications; that is why, in the previous subsection, it is not considered in comparisons. To include different bandwidth usage of these two modulations in our evaluation, an equal bandwidth scenario is also investigated.

In this scenario the bandwidth of the hybrid modulation is considered as the reference and the symbol rate of the DDMPSK is changed such that it occupies the same bandwidth as the hybrid method. Then, the modulations with equal bandwidth are compared (they may achieve different repetition orders while occupying the same bandwidth). According to (6.19) from section 6.3, for a symbol rate equal to $R_{S}^{R e f}=100$ Sym./s, combining 2FSK and 4FSK with MPSK (2FxP and $4 \mathrm{FxP}$ ), the null-to-null bandwidths become $3 R_{S}^{R e f}$ and $5 R_{S}^{R e f}$, respectively. MPSK modulation with a null-to-null bandwidth equal to $3 R_{S}^{R e f}$ and $5 R_{S}^{R e f}$ can have a symbol rate equal to $1.5 R_{S}^{R e f}$ and $2.5 R_{S}^{R e f}$, respectively (notice that a rectangular pulse shape is assumed and, therefore, PSK null-to-null bandwidth is twice the symbol rate). Using these higher symbol rates for MPSK, the number of transmitted symbols during $T_{P}^{R e f}$ is increased and this can be used to transmit redundant information in the same packet time.

So starting from a given PSK modulation scheme, the repetition order can be increased in two ways:

1. FSK in combination with PSK: It increases the bandwidth of the signal and the repetition order (symbol rate remains $R_{S}^{R e f}$ ).

2. MPSK with higher symbol rate (fast MPSK): This means that the raw bitrate is increased and more bits can be transmitted during $T_{P}^{R e f}$. This also increases bandwidth. 


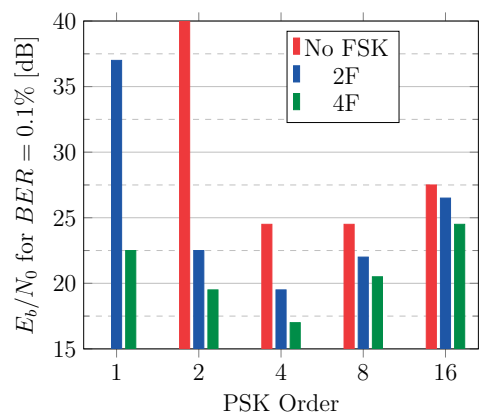

(a) Rayleigh with $D_{\max }=2 \mathrm{~Hz}$

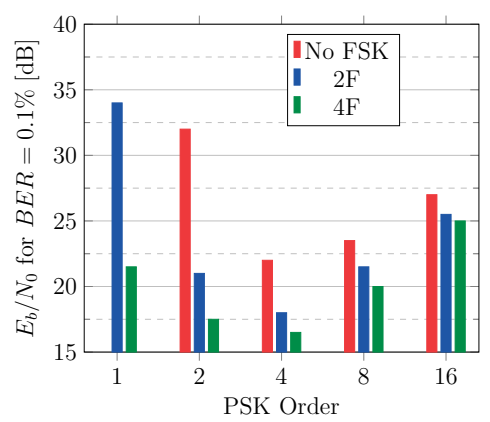

(c) Rice with $K=1$ and $D_{\max }=2 \mathrm{~Hz}$

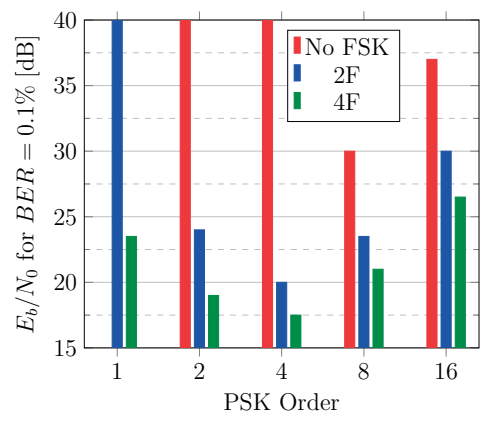

(b) Rayleigh with $D_{\max }=5 \mathrm{~Hz}$

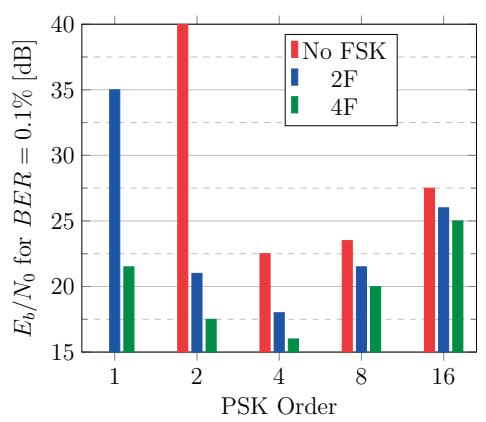

(d) Rice with $K=1$ and $D_{\max }=5 \mathrm{~Hz}$

Figure 6.7: $E_{b}^{\operatorname{Inf}} / N_{0}$ required for $B E R=0.1 \%$ in constant symbol rate scenario. The channel model is Rayleigh with Jakes Doppler spectrum for (a) and (b). The channel model is Rice with $K=1$ and Rounded Doppler spectrum for (c) and (d). The horizontal axis is the order of PSK and order equal to 1 means there is only FSK modulation.

With a symbol rate equal to $R_{S}$ for an MPSK modulated signal, the total number of transmitted symbols $\left(T X_{S y m}\right)$ during the reference packet time $\left(T_{P}^{R e f}\right)$ and the achieved $R O$ are as follows.

$$
\begin{gathered}
T X_{\text {Sym }}=T X_{\text {Inf }} \times\left(R_{S} / R_{S}^{R e f}\right) \\
R O=\frac{T X_{\text {Sym }} \times \log _{2}(M)}{T X_{\text {Inf }}}=\log _{2}(M) \times\left(R_{S} / R_{S}^{R e f}\right)
\end{gathered}
$$




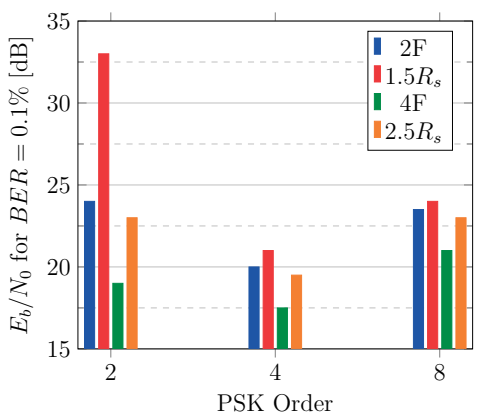

(a) Rayleigh with $D_{\max }=2 \mathrm{~Hz}$

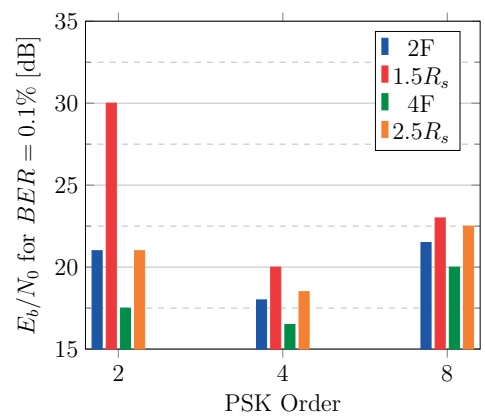

(c) Rice with $K=1$ and $D_{\max }=2 \mathrm{~Hz}$

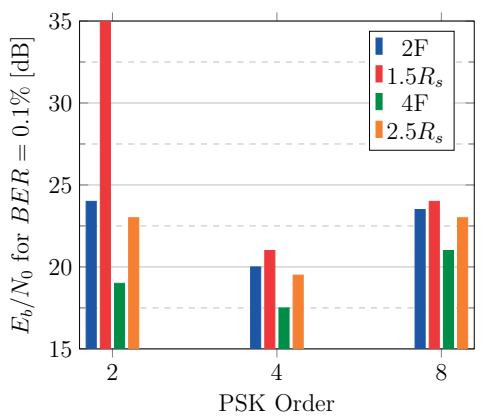

(b) Rayleigh with $D_{\max }=5 \mathrm{~Hz}$

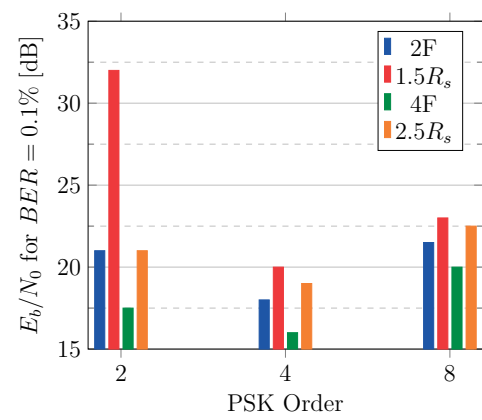

(d) Rice with $K=1$ and $D_{\max }=5 \mathrm{~Hz}$

Figure 6.8: $E_{b}^{\text {Inf }} / N_{0}$ required for $B E R=0.1 \%$ in equal bandwidth scenario. The channel model is Rayleigh with Jakes Doppler spectrum for (a) and (b). The channel model is Rice with $K=1$ and Rounded Doppler spectrum for (c) and (d). The horizontal axis is the order of PSK.

Remember that the symbol rate for FPSK remains $R_{S}^{R e f}$ and the repetition order is achieved using (6.47). Different combinations of LFSK and MPSK as well as different combinations of MPSK with higher symbol rates are shown in Table 6.4. The value in each cell of the table shows the repetition order that can be achieved with the corresponding combination.

For hybrid modulations in this scenario, the symbol rate does not change and will be the same as $R_{S}^{R e f}$. So a constant packet time (assumption 3) means that the number of symbols that are transmitted remains constant and equal to $T X^{I n f}$. To determine energy per transmitted symbol, similar reasoning as for constant symbol rate scenario can be used for the hybrid modulation in the equal bandwidth scenario. Thus, energy per transmitted bit $E_{b}$, is $E_{b}^{\operatorname{Inf}} / R O$ and energy per transmitted symbol (for hybrid modulation) is $E_{b}^{\text {Inf }}$. 
Table 6.4: Repetition order that can be achieved with each combination

\begin{tabular}{|c|c|c|c|c|}
\hline & 2FSK & $1.5 R_{\mathrm{S}}$ & 4FSK & $2.5 \mathrm{R}_{\mathrm{S}}$ \\
\hline 2PSK & 2 & 1.5 & 3 & 2.5 \\
\hline 4PSK & 3 & 3 & 4 & 5 \\
\hline 8PSK & 4 & 4.5 & 5 & 7.5 \\
\hline
\end{tabular}

In case of MPSK modulation with higher symbol rate (fast MPSK), the energy per transmitted symbol is derived as follows. For a repetition order $R O$, the energy per transmitted bit is $E_{b}^{I n f} / R O$. Each transmitted symbol includes $\log _{2}(M)$ bits so the energy per transmitted symbol $E_{S}$ is:

$$
E_{S}=\log _{2}(M) \times \frac{E_{b}^{I n f}}{R O},
$$

Substituting $R O$ from (6.49) into (6.50), $E_{S}$ is obtained.

$$
E_{S}=E_{b}^{\operatorname{Inf}} \times \frac{R_{S}^{R e f}}{R_{S}}
$$

This is used as symbol energy in the simulations which guarantees that the energy per information bit remains constant. The results are shown in terms of $E_{b}^{\operatorname{Inf}}$. There are three factors which affect the BER performance in this scenario; 1) repetition order obtained using the same bandwidth, 2) the energy per transmitted symbol and 3) the effect of temporal fading which is proportional to $D_{\max } T$ and depends on symbol rate variations.

The consequence of a higher symbol rate in case of MPSK is that the energy per symbol $\left(E_{S}\right)$ is lower (see (6.51)). This makes the redundant information transmitted using higher symbol rate PSK less effective. On the other hand, the effect of temporal fading is proportional to $D_{\max } T$ (the maximum Doppler shift normalized to the symbol rate) which is an advantage for higher symbol rate PSK compared to hybrid modulation (the symbol rate remains constant for hybrid modulation). An increased symbol rate has a similar effect as decreasing the $D_{\max }{ }^{1}$.

For an overview of different cases in the equal bandwidth scenario, the $E_{b}^{\operatorname{Inf}} / N_{0}$ required to achieve $B E R=0.1 \%$ is shown in Figure 6.8 (the BER curves for different combinations and channel models can be found in Appendix D). The

\footnotetext{
${ }^{1}$ The effect of increasing the symbol rate to $1.5 R_{S}^{R e f}$ and $2.5 R_{S}^{R e f}$ on $D_{\max } T$ is similar to decreasing $D_{\max }$ to $D_{\max } / 1.5$ and $D_{\max } / 2.5$ when the symbol rate is constant.
} 
horizontal axis in the diagrams shows the PSK order while each bar shows either the combination of that PSK order with FSK (blue and green bars) or using solely PSK with a higher symbol rate (red and orange bars). Similar to the constant symbol rate scenario, 4PSK performs the best among all PSK orders. Furthermore, a combination with FSK needs less $E_{b}^{I n f} / N_{0}$ compared to fast PSK in case of equal bandwidth occupation. The difference is not large in a Rayleigh channel and particularly for combinations with 2FSK; however, it grows when a combination with 4FSK or a Rician channel are considered. Similar to the constant symbol rate scenario, among the considered combinations, 4F4P performs the best with (at least) around $2 \mathrm{~dB}$ improvement compared to $2.5 R_{S}$ MPSK.

\subsection{CONCLUSION}

The effects of a time-varying channel in combination with Carrier Frequency Offset (CFO) were considered simultaneously for ultra-narrowband (UNB) communications. An offset tolerant demodulator was proposed for a general case of hybrid LFSK/MPSK modulation using Differential LFSK and Double Differential MPSK (DDMPSK). Hybrid modulation increases the raw bit-rate in a more power efficient manner compared to higher order MPSK. The increased bit-rate can be exploited by introducing channel coding and diversity techniques to improve the performance of a UNB system in time-varying fading channels. The higher bit-rate achieved by hybrid modulation was used for a simple repetition of the information packet during the same packet time. The system was simulated in Rayleigh and Rician channels with maximum Doppler shifts equal to $2 \mathrm{~Hz}$ and $5 \mathrm{~Hz}$. The BER performance of the proposed demodulator was compared with an offset tolerant Double Differential MPSK demodulator in different scenarios. It was shown that at $\mathrm{BER}=0.1 \%$, a hybrid modulation scheme performs better than the MPSK modulation in all cases by at least $1 \mathrm{~dB}$ and up to more than $10 \mathrm{~dB}$ depending on the modulation order, scenario, channel model and maximum Doppler shift. Using a simple repetition coding and time diversity, a combination of 4FSK and 4PSK (4F4P) is the best combination among the investigated modulations. Considering all cases, $4 \mathrm{~F} 4 \mathrm{P}$ requires $2 \mathrm{~dB}$ less $E_{b} / N_{0}$ than the best DDMPSK at BER $=0.1 \%$. In this work, repetition coding was used for its simplicity. The BER performance and complexity trade-offs, when using other coding schemes, requires further study. Moreover, using the proposed hybrid demodulator in combination with frequency diversity is an interesting direction for future research. 


\section{7 \\ CONCLUSION}

Low Power Wide Area Networks (LPWANs) are an emerging type of wireless networks which realize long range and low data rate communication for IoT applications. Ultra-Narrowband (UNB) communication is one of the interesting technologies for implementation of LPWAN. It uses unlicensed spectrum, offers robustness against interference and provides low cost communication with a wide coverage. Although the very low data rate in UNB communication relaxes some aspects of the transceiver design (such as lower clock frequencies in the baseband processing), it poses other challenges. The extremely narrow bandwidth of the signal in UNB communications (in the order of a few hundred hertz) makes UNB susceptible to Carrier Frequency Offset (CFO) and temporal fading.

A mismatch between the transmitter and the receiver oscillators or the movement of the transceiver nodes relative to each other can cause CFO. Offset tolerant demodulators have been proposed in literature as a solution to the problem of $\mathrm{CFO}$ and as an alternative for precise but power hungry carrier frequency recovery. However, most of these methods are designed for cases where the frequency offset is in the order of the signal bandwidth or even smaller than that. Such a value for CFO in a UNB communication system requires very precise crystals with thermal compensation which are not cost and energy efficient.

Temporal fading is the variation of the channel during transmission time. The challenge of a time-varying fading channel is more severe for UNB communication due to their long symbol periods and transmission times. Even if wireless nodes are fixed, which is usually the case for the UNB communication systems, movement of the scatterers of the signal (surrounding objects) leads to a time-varying channel. Time and frequency diversity techniques combined with channel coding and interleaving can be utilized to improve the reliability of the communication in a time-varying fading channel. Nevertheless, these schemes require transmitting redundant bits which makes the transmission time in a UNB system even longer.

This thesis focused on addressing the above mentioned challenges in the down- 
link of a UNB system using offset tolerant demodulators. This chapter summarizes the contributions and presents some possible directions for future research. First, in the next section the contributions are summarized and the research questions introduced in Chapter 1 are answered. Then, recommendations for future research are proposed for further investigation.

\subsection{Summary}

\subsubsection{Offset Tolerant Demodulators for UNB}

As mentioned earlier, most of the offset tolerant demodulators assume that the $\mathrm{CFO}$ is in the order of the signal bandwidth or even smaller. In case of UNB communication, such a CFO still needs high precision carrier recovery and costly crystals. Therefore, to combat the CFO while using low cost crystals and avoiding high precision carrier recovery, offset tolerant demodulators for UNB need to tolerate very large frequency offsets. That is why the first research question presented in Chapter 1 was defined as follows.

1. What are the limitations of offset tolerant demodulators, which can tolerate large frequency offset, for UNB communications?

The answer to this question is sought in Chapter 3 where we considered two different modulation schemes that are mostly used in UNB communications, namely, FSK and PSK. The goal was to investigate demodulators which can tolerate arbitrarily large frequency offsets given that the low-pass filter before the demodulator captures the signal properly in the presence of CFO. The BER performance and the complexity of the two demodulators were investigated to find out their limitations when large CFO tolerance is required.

Among various FSK demodulators, a demodulator based on the Discrete Fourier Transform (DFT) was selected as the one with potential to tolerate large frequency offset. Therefore, a DFT-based demodulator was designed for BFSK using a simple detection algorithm. An existing DFT-based window synchronization scheme was used for the DFT-based demodulator. This synchronization scheme is necessary to tolerate large frequency offset and in our design it is also used to determine the bins of the DFT corresponding to the transmitted BFSK frequencies.

For PSK modulation an autocorrelation based demodulator (ACD) was used. Using Double Differential encoding for PSK and ACD as demodulator, the decision metric will be independent of the frequency offset value; thus, a Double Differential PSK signal can be detected in the presence of a relatively large frequency offset (multiple time the symbol rate). To have a fair comparison between the complexity of the FSK and PSK demodulators, a synchronization algorithm was designed and used for PSK which is independent of the frequency offset.

The BER performance of these two demodulators, when a large offset tolerance is needed, were evaluated. In the presence of a large CFO, a wide low-pass filter 
is required prior to the demodulators so that the signal can be captured. This increases the noise power at the input of the demodulator. It was shown that the BER performance of DDPSK degrades as a consequence of the increased noise power. The simulation results showed that the $E_{b} / N_{0}$ required for $B E R=0.1 \%$ increases by $2 \mathrm{~dB}$ when the range of tolerable offset changes from around $\pm 3 R_{S}$ to $\pm 15 R_{S}(N=8$ and $N=32)$. In contrast, the BER performance of the DFTbased demodulator is not affected by the bandwidth of the low-pass filter. For the DFT-based demodulator the DFT works as a filter bank. Since the number of DFT points increases when the tolerable offset increases, the bandwidth of each of these filters remains constant independent of the filter before the demodulator. Thus, the BER performance of the DFT-based demodulator does not change.

The complexity of the two demodulators was calculated in terms of complex multiplications and additions required for synchronization and symbol detection. It was shown that the complexity of the DDPSK increases linearly with the number of samples per symbol, $N$. On the other hand, the complexity of the DFT-based demodulator is in the order of $N^{2} \log _{2}(N)$ which is considerably larger than the complexity of the DDPSK demodulator. The number of samples per symbol is proportional to the range of tolerable frequency offset; therefore, when the range of tolerable frequency offset increases, the complexity of the DFT-based demodulator increases drastically. As an example, the complexity of the DFT-based demodulator for each symbol detection (assuming a packet length of 250 symbols) becomes 20 times larger when the range of tolerable offset changes from $\pm 3 R_{S}$ to $\pm 15 R_{S}(N=8$ and $N=32)$.

The simulation results and discussions showed that a DDPSK demodulator is limited by its BER performance degradation while the DFT-based demodulator can lead to a drastic increase of complexity when the range of offset tolerance increases. To enable large offset tolerance, these limitations need to be overcome.

\subsubsection{Scalable Offeset Tolerance}

The scalable offset tolerance was introduced as one of the main targets of this work. As defined in Chapter 2, scalable offset tolerance means achieving the same BER performance for a certain $E_{b} / N_{0}$ independent of the CFO. According to the previous subsection, the investigated offset tolerant demodulators for PSK and FSK both have limitations in presence of arbitrarily large frequency offset. The BER performance of the DDPSK demodulator degrades when a large offset tolerance is required. On the other hand, the complexity of the DFT-based demodulator extremely increases if a larger offset tolerance is required. These limitations raised the second research question:

2. How can we circumvent the limitations of the demodulators to achieve scalable offset-tolerance?

The solutions for demodulator limitations were discussed in Chapter 4 and Chapter 5 and are summarized in the following. 


\section{Shifted correlation for DDPSK}

In Chapter 4, the BER performance of the autocorrelation demodulator (ACD) for DDPSK in the presence of a large frequency offset was considered. Although DDPSK is able to tolerate frequency offset, the wide filter required for a large offset tolerance includes more noise which leads to BER performance degradation. To find a solution, first, the problem was defined by investigating the ACD and the noise distribution at its output. It was shown that the main reason for dependence of the BER performance on the filter bandwidth is the multiplication of noise components in the first autocorrelation stage.

To overcome this problem, a shifted correlation method was proposed in Chapter 4. It is assumed that the sampling frequency at the output of the filter and the input of the demodulator satisfies the Nyquist criterion with respect to the filter bandwidth. Thus, when the filter is wider than the signal bandwidth the symbols are oversampled. The proposed method exploits this oversampling by introducing additional correlation paths to the conventional DDPSK demodulator. In each path, there are two stages similar to the conventional demodulator. In the first stage, the $n^{\text {th }}$ symbol is correlated with a shifted version of the samples of the $(n-1)^{t h}$ symbol. The result of this stage passes through another differential decoder. The outputs of these paths are added together and this sum is used to make the final decision. As shown in Chapter 4, the proposed method increases the SNR at the input of the detector and compensates for the SNR loss resulting from the increased filter bandwidth. Therefore, the BER performance remains almost unchanged for all values of the filter bandwidth. The simulation results revealed that the proposed method leads to more than $2 d B$ improvement in BER performance when the bandwidth of the filter is 16 times the symbol rate (which implies that the offset tolerance is around $\pm 7 R_{S}$ ). For wider filters i.e. wider range of frequency offset tolerance, this gain increases further.

\section{Low complexity window synchronization for FSK}

It was shown in Chapter 3 that the main drawback of the DFT-based offset tolerant demodulator is its complexity. The high complexity stems from the window synchronization algorithm which is used for this demodulator. The window synchronization algorithm is necessary for the proper functioning of the demodulator; however, its complexity is prohibitive for tolerating a large frequency offset. The existing synchronization algorithm for this demodulator is complex as it involves calculating the DFT of a sliding window called Sliding DFT (SDFT).

A variety of algorithms have been proposed for efficient implementation of the SDFT. In our literature review in Chapter 5, these algorithms were divided into two categories, Complete SDFT (C-SDFT) and Single Bin SDFT (SB-SDFT). The former class computes all bins of the DFT altogether while the latter can be used to calculate each bin separately. A review on the SDFT calculating 
structures in Chapter 5 showed that if the number of calculated bins of an $N$ point DFT is sufficiently small, the SDFT can be implemented efficiently using SB-SDFT structure.

In light of these facts, a new synchronization algorithm was proposed where the number of the DFT bins required for synchronization is reduced. Actually, due to zero-padding and oversampling, only a limited number of bins called the Bins of Interest (BoI) include signal information which is explained in Chapter 5. To find the BoI, a step-by-step zooming algorithm was proposed. Furthermore, the DFT-based demodulator introduced in Chapter 3, needs zero-padding for proper performance and the synchronization algorithm should also use zero padding for correct operation. However, existing SB-SDFT structures do not consider zero-padding. That is why a modified version of SB-SDFT was also proposed in Chapter 5 to incorporate zero padding. The stability of the proposed SB-SDFT method was also considered and a stable version using a damping factor was introduced.

Using the proposed algorithm and its implementation based on the proposed SB-SDFT calculator, the complexity increases slower compared to the conventional synchronization algorithm when the range of tolerable $\mathrm{CFO}$ increases. It was shown that for an oversampling factor of eight the proposed algorithm obtains $68 \%, 48 \%, 61 \%$ saving in the number of complex additions, complex multiplications and memory compared to the conventional synchronization algorithm while achieving the same BER performance. Based on simulation results presented in Chapter 5, for an oversampling factor of 32, the numbers of complex multiplications and complex additions are reduced by more than $85 \%$ (and memory more by $90 \%$ ). This oversampling factor corresponds to an offset tolerance about \pm 14.5 times the symbol rate.

\subsubsection{Offeset Tolerance and Temporal Fading}

As mentioned earlier in this chapter, temporal fading is another problem which must be considered in UNB communication. The third research question was focused on addressing CFO and temporal fading simultaneously.

3. How can we handle the temporal fading in UNB communication and achieve the required redundancy for diversity while using offset tolerant demodulators?

Using diversity techniques is a solution to temporal fading; however, it requires transmitting redundant information. Thus, we either need to increase the raw bit-rate or increase the transmission time. A longer transmission time means that the RF part is on for a longer time which increases power consumption. On the other hand, increasing the bit-rate using higher order PSK degrades the BER performance while using higher order FSK increases the bandwidth.

A third solution to increase the raw bit-rate is hybrid frequency/phase modulation which has been proposed in literature. It changes the power and band- 
width efficiency trade-off by providing more bandwidth efficiency than higher order FSK and more power efficiency (from a BER performance perspective) compared to higher order PSK. The increased bit-rate can be exploited by introducing channel coding and diversity techniques to improve the performance of a UNB system in time-varying fading channels.

To tolerate $\mathrm{CFO}$, an offset tolerant hybrid demodulator was proposed in chapter 6. Different combinations of FSK and PSK orders were simulated using AWGN channels. It was shown that for the combination of BFSK and QPSK, the proposed demodulator can achieve $6 \mathrm{~dB}$ improvement at $B E R=0.1 \%$ compared to DD8PSK which provides the same modulation order (3 bits per symbol).

To alleviate the effect of temporal fading, a time diversity scheme was proposed using the offset tolerant hybrid demodulator. The increased bit-rate achieved by hybrid modulation was used for re-transmitting the information bits during the same packet time. The hybrid demodulator was simulated for different combinations of FSK and PSK. Rayleigh and Rician channel models were considered with maximum Doppler shifts equal to $2 \mathrm{~Hz}$ and $5 \mathrm{~Hz}$. Two different scenarios were considered for simulations: a constant symbol rate scenario and an equal bandwidth scenario.

It was shown that at $B E R=0.1 \%$, a hybrid modulation scheme performs better than higher order DDPSK modulation in all cases by at least $1 \mathrm{~dB}$ and up to more than $10 \mathrm{~dB}$ depending on the modulation order, scenario, channel model and maximum Doppler shift. Using simple repetition coding and time diversity, a combination of 4FSK and 4PSK (4F4P) is the best combination among the investigated modulations. Considering all cases, at $B E R=0.1 \%, 4 \mathrm{~F} 4 \mathrm{P}$ needs $2 \mathrm{~dB}$ less $E b / N_{0}$ than the best Double Differential MPSK.

\subsection{Main Contributions}

The main contributions of this thesis can be summarized as follows:

»We investigated the performance and complexity of two offset tolerant demodulators for PSK and FSK which are capable of tolerating frequency offsets larger than the signal bandwidth. A DFT-based demodulator was designed for FSK and an autocorrelation based double differential demodulator was considered for PSK. The evaluations clarified the limitations of these methods which were addressed in the rest of the work (Chapter 3 ).

»We developed and evaluated a shifted correlation scheme for DDPSK demodulation. It alleviates the BER performance loss of the conventional autocorrelation based DDPSK demodulator in the presence of a wide filter and large frequency offset (Chapter 4).

»We developed and evaluated a low complexity synchronization algorithm for the DFT-based demodulator. This algorithm uses a subset of the DFT bins for synchronization. For efficient implementation of the proposed 
synchronization algorithm a Single Bin Sliding DFT (SB-SDFT) structure with zero-padding was designed as well (Chapter 5 ).

»We developed and evaluated an offset tolerant demodulator for hybrid frequency/phase modulation and designed a system based on time-diversity to overcome temporal fading. The hybrid modulation was utilized to increase the raw bit-rate which was exploited to re-transmit the information packet during the same packet time. This system exploits the time-diversity which is provided by a time-varying channel and improves the BER performance (Chapter 6).

\subsection{Recommendations FOR Future WORK}

In this thesis, we mainly focused on a constant frequency offset. If the variations in frequency offset remain sufficiently small, the proposed demodulator will not be affected. For the DFT-based demodulator a variable CFO causes deviation of FSK frequencies from the DFT bins determined during synchronization. These variations can be tolerated as far as they remain smaller than the bin resolution of the DFT during the whole packet. For DDPSK the variable offset can be tolerated as far as the change in the $\mathrm{CFO}$ during three symbols remains negligible. For UNB applications a largely time-varying CFO is not very likely as the transmitter and the receiver are mainly fixed. Nevertheless, it is interesting to look for techniques to complement the proposed demodulators for low data rate applications where largely time-varying CFO exists. For the DFT-based demodulator, the adjacent bins of the bins selected in the synchronization phase can be monitored during the packet. In this way, using a decision directed scheme, if the adjacent bins have a higher average value during a few symbols, they can be used as new bins for decision making. For the DDPSK, the linear variation in frequency offset shows itself as a constant phase at the output of the second stage. To solve this, a decision directed method might be used to correct for this phase offset and avoid performance loss.

As another solution for temporal fading, a frequency diversity technique can be used. It may use a hybrid modulation scheme and in addition to transmitting signal over different time periods, it can transmit redundant information using different carrier frequencies (a sort of frequency hopping scheme). If the differences between frequencies are selected carefully and are larger than the coherence bandwidth of the channel, this will add diversity and can improve performance of the system. Although leveraging frequency diversity e.g. using frequency hopping adds complexity, it is an interesting choice when the time variation of a channel is too slow to provide the required diversity.

In the proposed scheme for temporal fading (Chapter 6), the repetition coding scheme was used. Although it offers considerable simplicity, its ability to improve performance is limited. It is anticipated that a better BER performance can be achieved if a more complicated channel coding scheme is used. Since the 
processing power of the communication node in the downlink is limited, the trade-off between the decoder complexity and the performance improvement must be evaluated. On the other hand, the short packet length affects the performance of most channel coding schemes as they are usually optimized for long packets. Finding a proper coding scheme for a short block length is an active research topic in the field of Ultra-Reliable Low Latency (URLL) communications for 5G. This formulates an interesting question for future research on finding low code-rate and simple channel coding schemes which are suitable for the proposed system.

In this work we focused on an offset tolerant demodulator and how it can handle frequency offset in the physical layer. It would be interesting to look into the opportunities that such offset tolerant demodulators provide in the design of the MAC layer and network layer. In UNB communication systems such as Sigfox, the downlink communication is dependent on an uplink initiation. One of the reasons for this approach is the frequency uncertainty in the communication node. If an offset tolerant demodulator is used, the restrictions on the downlink communication might be relaxed. On the other hand, if the fading problem can be overcome efficiently using a diversity scheme (such as the one proposed in Chapter 6), the reliability of the downlink communication in a UNB network increases. Nevertheless, figuring out how this can be exploited to improve the overall capacity and/or reliability of a UNB network, needs further investigation of the MAC- and network layers. 


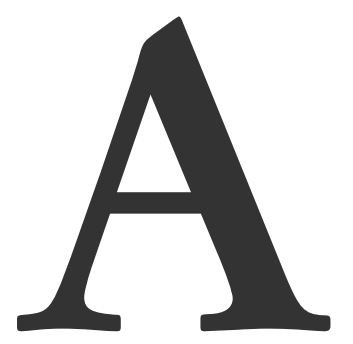

\section{Mathematical Proof for $k_{c}$ Calculation Algorithm}

According to the explanation in Chapter 5, to achieve $k_{c}^{\gamma}$ at each step, the magnitudes of DFTs for a sliding window with $2 N$ one-sample shifts are added. Then, $k_{c}^{\gamma}$ is the bin for which this sum has a maximum value (see (5.9) and (5.10)). In this appendix, it is mathematically shown that this sum over DFT magnitudes has a maximum at the center frequency. This is correct as long as all the $2 \mathrm{~N}$ windows are in the preamble. The first window can start in any part of either a one symbol or a zero symbol in the preamble.

As the zero-padding factor changes during the zoom stage, (5.9) and (5.10) must be shown for the general case of all zero-padding factors in different steps of the zooming algorithm. For this purpose the concept of the Discrete Time Fourier Transform (DTFT) is used here. DTFT is a version of the Fourier Transform which maps discrete time samples to a continuous frequency domain. The DTFT of a discrete function in the time domain, shown by $x[n]$, is denoted by $X(\Omega)$ where $\Omega=2 \pi f / F_{s}(-\pi<\Omega<\pi)$ is the frequency normalized to the sampling frequency, $F_{s}$. The $k^{t h}$ bin of the DFT calculated for $N$ samples with zeropadding factor $I$, actually is the value $X(\Omega)$ of DTFT for effectively $N$ samples, sampled at $f=(k / N I) F_{s}(\Omega=2 \pi k / N I)$ [82]. Using the concept of the DTFT, (5.9) and (5.10) can be written as follows.

$$
\Omega_{c}=\max _{\Omega \in[-\pi .+\pi]} \mathscr{X}(\Omega)
$$

where:

$$
\mathscr{X}(\Omega)=\sum_{i=0}^{2 N-1}\left|X_{i}(\Omega)\right|^{2}
$$

$X_{i}(\Omega)$ is the DTFT for the $i^{t h}$ window and $\Omega_{c}=2 \pi f_{c} / F_{s}=\omega_{c} T_{s}$. Because (A.1) and (A.2) are generalized versions of (5.9) and (5.10), which are true for all zero- 


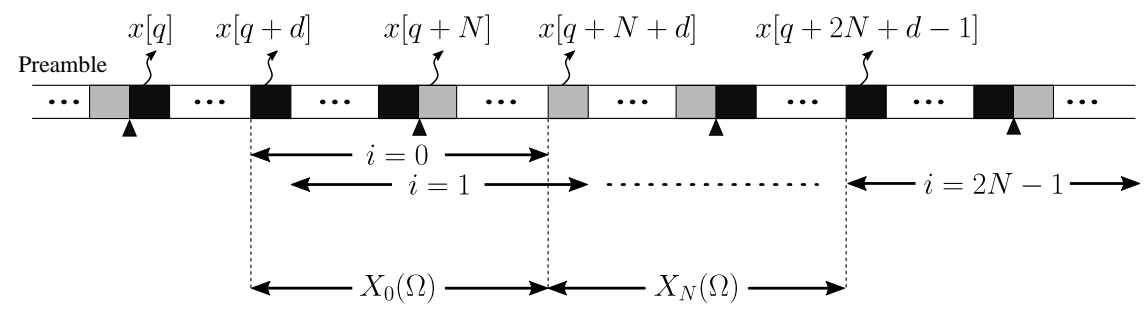

$\square$ Sample of Odd Symbols $\left(f_{1}\right) \square$ Sample of Even Symbols $\left(f_{0}\right)$

Figure A.1: A part of the preamble and the windows used for calculating $X_{k, i}^{\gamma}$ in the zoom stage. Three complete symbols $(1,0,1)$ with a part of the symbol before the first one and a part of the symbol after the last one are shown. The boundaries of the symbols are shown by triangle markers. Black and grey squares show samples of Odd and Even symbols, respectively. Double-headed arrows are windows for which the DFT or the DTFT are calculated. $i=0$ to $i=2 N-1$ are the windows used for the sum in (5.10). The two windows shown by $X_{0}(\Omega)$ and $X_{N}(\Omega)$ are windows used for the mathematical proof of the algorithm.

padding factors, the superscript $\gamma$ from (5.10) is removed in (A.2). If it is proved that $\mathscr{X}(\Omega)$ has a maximum at $\Omega_{c}$, then, the sum in $(5.10)$ has a maximum at the bin closest to $f_{c}$ (called $k_{c}$ ) for each zero-padding factor. This can be concluded considering that the DFT values for each $k$ and window are actually samples of the DTFT.

\section{A.1 Calculating the Components of $\mathscr{X}(\Omega)$}

The DTFT of a signal $x[n]$ over $N$ samples is as follows.

$$
X(\Omega)=\sum_{n=0}^{N-1} x[n] e^{-j \Omega n}, \Omega=2 \pi f / F_{s}
$$

Fig. A.1 depicts a part of the preamble which is used for mathematical derivation. Black squares are the samples of an Odd symbol $\left(f_{1}\right)$ and gray ones belong to an Even symbol $\left(f_{0}\right)$. Each double-headed arrow stands for a window (set of samples) of length $N$ which is used for calculating the DTFT. In general, during the zoom stage, the window is not synchronized yet. Thus, the set of sliding windows in (A.2) does not necessarily start from the beginning of a symbol. To account for this, it is assumed that the first window from the set of $2 N$ windows starts from a window with delay $d$ from the beginning of a symbol. This is shown with $i=0$ in Fig. A.1. The first sample of that symbol is shown by $x[q]$. The following procedure is executed considering windows $i=0$ to $i=2 N-1$ and calculating the sum of the magnitudes of the DTFTs for these windows. The 
window $i=0$ starts in an Odd symbol; nevertheless, the same mathematical formulation can be achieved if the black and gray samples are interchanged in Fig. A.1 i.e. if $i=0$ starts in an Even symbol. This generalization is possible because of the specific frequency separation for BFSK which is equal to the symbol rate.

Now, let us consider a pair of windows in the summation of (A.2) with delay $d$ of the odd symbol $\left(f_{1}\right)$ and the same delay for the next even symbol $\left(f_{0}\right)$ (i.e. $i=0$ and $i=N$ in (A.2)). In Fig. A.1 these are shown by $X_{0}(\Omega)$ and $X_{N}(\Omega)$. If $G_{0}(\Omega)$ is defined as follows:

$$
G_{0}(\Omega)=\left|X_{0}(\Omega)\right|^{2}+\left|X_{N}(\Omega)\right|^{2}
$$

Then, $\mathscr{X}(\Omega)$ can be derived in terms of $N$ similar pairs and different $G_{m}$ as:

$$
\mathscr{X}(\Omega)=\sum_{m=0}^{N-1} G_{m}(\Omega)
$$

where $G_{m}(\Omega)=\left|X_{m}(\Omega)\right|^{2}+\left|X_{m+N}(\Omega)\right|^{2}$. First, $G_{0}(\Omega)$ is analyzed. Considering BFSK modulation, the samples for the $i=0$ and $i=N$ window are as follows.

$$
\begin{gathered}
\mathbf{x}_{0}[n]= \begin{cases}e^{j\left(\omega_{1}(n+d+q) T_{s}\right)} & 0 \leq n<N-d \\
e^{j\left(\omega_{0}(n+d+q-N) T_{s}+\theta_{1}\right)} & N-d \leq n<N\end{cases} \\
\mathbf{x}_{N}[n]= \begin{cases}e^{j\left(\omega_{0}(n+d+q) T_{s}+\theta_{1}\right)} & 0 \leq n<N-d \\
e^{j\left(\omega_{1}(n+d+q-N) T_{s}+\theta_{0}+\theta_{1}\right)} & N-d \leq n<N\end{cases}
\end{gathered}
$$

where $\omega_{1}$ and $\omega_{0}$ are $2 \pi f_{1}$ and $2 \pi f_{0}$, respectively. In (A.6) and (A.7), $\theta_{1}=$ $\omega_{1} N T_{s}$ and $\theta_{0}=\omega_{0} N T_{s}$ are actually accumulated phase at the end of an Odd symbol and Even symbol, respectively. Without loss of generality, it is assumed that the initial phase before $x[q]$ (in Fig. A.1) is zero. Using $\mathbf{x}_{0}[n], \mathbf{x}_{N}[n]$ and the definition of the DTFT in (A.3), $X_{0}(\Omega)$ and $X_{N}(\Omega)$ are calculated.

$$
\begin{aligned}
X_{0}(\Omega)= & \sum_{n=0}^{N-d-1} e^{j\left(\omega_{1} T_{s}(n+d+q)-\Omega n\right)} \\
& +\sum_{n=N-d}^{N-1} e^{j\left(\omega_{0} T_{s}(n+d+q)-\Omega n\right)} \\
X_{N}(\Omega)= & \sum_{n=0}^{N-d-1} e^{j\left(\omega_{0} T_{s}(n+d+q)+\omega_{1} N T_{s}-\Omega n\right)} \\
& +\sum_{n=N-d}^{N-1} e^{j\left(\omega_{1} T_{s}(n+d+q)+\omega_{1} N T_{s}-\Omega n\right)}
\end{aligned}
$$


Notice that in (A.6) and (A.7), $\theta_{1}-\omega_{0} N T_{s}$ and $\theta_{0}-\omega_{1} N T_{s}$ are equal to $+\left(\omega_{1}-\right.$ $\left.\omega_{0}\right) N T_{s}$ and $-\left(\omega_{1}-\omega_{0}\right) N T_{s}$, respectively. Remember from Chapter 3 and Chapter 5 that the frequency separation of BFSK is equal to the symbol rate $R_{\text {sym }}=1 / T$ and $T_{s} / T=1 / N$. So $\pm\left(\omega_{1}-\omega_{0}\right) N T_{s}$ are removed from the phase of the signals since they equal $\pm 2 \pi$. Now that the primary expressions for $X_{0}(\Omega)$ and $X_{N}(\Omega)$ are obtained, a new variable is introduced to simplify the rest of the proof.

\section{A.2 Definition of $\alpha$}

The main target is to prove that $\mathscr{X}(\Omega)$ has a maximum at $\Omega_{c}=\omega_{c} T_{s}$. Since the frequency separation of BFSK is equal to the symbol rate $\left(1 / T=1 / N T_{s}\right), \omega_{1} T_{s}$ and $\omega_{0} T_{s}$ can be written in terms of center frequency $\omega_{c}$ as follows.

$$
\omega_{0} T_{s}=\omega_{c} T_{s}-\pi / N, \omega_{1} T_{s}=\omega_{c} T_{s}+\pi / N,
$$

Now that $\omega_{0} T_{s}$ and $\omega_{1} T_{s}$ are written in the form of deviations from $\omega_{c} T_{s}$, let us define $\alpha$ as the deviation of $\Omega$ from $\Omega_{c}$.

$$
\alpha=\Omega-\omega_{c} T_{s}
$$

In the following, the variable $\Omega$ is changed to $\omega_{c} T_{s}+\alpha$ and (A.4), (A.5), (A.8) and (A.9) are formulated as functions of $\alpha$. These new functions are denoted by a tilde over their name (for instance $\mathscr{X}(\Omega)$ changes to $\tilde{\mathscr{X}}(\alpha)$ ). When all functions are formulated in terms of $\alpha$, instead of proving that there is a maximum at $\Omega=\Omega_{c}$ for $\mathscr{X}(\Omega)$, it must be proved that $\tilde{\mathscr{X}}(\alpha)$ has a maximum at $\alpha=0$. This simplifies the calculations, particularly, it enables us to eliminate the terms related to $\omega_{c} T_{s}$ which is seen in the expressions for $\omega_{1} T_{s}$ and $\omega_{0} T_{s}$ (A.10) as well.

By change of variable to $\alpha$, (A.5) can be written as:

$$
\tilde{\mathscr{X}}(\alpha)=\sum_{m=0}^{N-1} \tilde{G}_{m}(\alpha)
$$

For now, we focus on $\tilde{G}_{0}(\alpha)=\left|\tilde{X}_{0}(\alpha)\right|^{2}+\left|\tilde{X}_{N}(\alpha)\right|^{2}$. Substituting $\omega_{1} T_{s}$ and $\omega_{0} T_{s}$ from (A.10) to (A.8) and (A.9) while replacing $\Omega$ with $\omega_{c} T_{s}+\alpha$ we have:

$$
\begin{aligned}
\tilde{X}_{0}(\alpha)= & e^{j\left(\omega_{c} T_{s}+\frac{\pi}{N}\right)(d+q)} \sum_{n=0}^{N-d-1} e^{j\left(\frac{\pi}{N}-\alpha\right) n} \\
& +e^{j\left(\omega_{c} T_{s}-\frac{\pi}{N}\right)(d+q)} \sum_{n=N-d}^{N-1} e^{-j\left(\frac{\pi}{N}+\alpha\right) n}
\end{aligned}
$$




$$
\begin{aligned}
\tilde{X}_{N}(\alpha)= & -e^{j\left[\left(\omega_{c} T_{s}-\frac{\pi}{N}\right)(d+q)+\omega_{c} T\right]} \sum_{n=0}^{N-d-1} e^{j-\left(\frac{\pi}{N}+\alpha\right) n} \\
& -e^{j\left[\left(\omega_{c} T_{s}+\frac{\pi}{N}\right)(d+q)+\omega_{c} T\right]} \sum_{n=N-d}^{N-1} e^{j\left(\frac{\pi}{N}-\alpha\right) n}
\end{aligned}
$$

where $T=N T_{s}$ and the terms which are independent of $n$ are taken out of summation. As a result of the change of variable, $\pi$ appears in the phase of the exponential functions in (A.9) which is converted to a negative sign before the summations in (A.14). For a complex function $f(x),|f(x)|^{2}=f(x) f(x)^{*}$ where $f(x)^{*}$ is the complex conjugate of $f(x)$. Thus, the magnitudes of $\tilde{X}_{0}(\alpha)$ and $\tilde{X}_{N}(\alpha)$ are as follows.

$$
\begin{aligned}
\left|\tilde{X}_{0}(\alpha)\right|^{2}= & \sum_{n, p=0}^{N-d-1} e^{j\left(\frac{\pi}{N}-\alpha\right)(n-p)}+\sum_{n, p=0}^{d-1} e^{-j\left(\frac{\pi}{N}+\alpha\right)(n-p)} \\
& +2 \sum_{n=0}^{N-d-1} \sum_{p=N-d}^{N-1} \cos (\Theta(n, p)-\alpha(n-p)) \\
\left|\tilde{X}_{N}(\alpha)\right|^{2}= & \sum_{n, p=0}^{N-d-1} e^{-j\left(\frac{\pi}{N}+\alpha\right)(n-p)}+\sum_{n, p=0}^{d-1} e^{j\left(\frac{\pi}{N}-\alpha\right)(n-p)} \\
& +2 \sum_{n=0}^{N-d-1} \sum_{p=N-d}^{N-1} \cos (-\Theta(n, p)-\alpha(n-p))
\end{aligned}
$$

where $\Theta(n, p)=\frac{\pi}{N}(2(d+q)+n+p)$ and the following equality is used:

$$
\sum_{m, k=0}^{M-1} a_{m} b_{k}=\sum_{m, k=0}^{M-1} a_{k} b_{m}=\left(\sum_{m=0}^{M-1} a_{m}\right)\left(\sum_{m=0}^{M-1} b_{m}\right)
$$

The first summation of (A.16) and the second summation of (A.15) can be rewritten as follows.

$$
\begin{gathered}
A(\alpha)=\sum_{n, p=0}^{N-d-1} e^{-j\left(\frac{\pi}{N}+\alpha\right)(n-p)}=\sum_{n, p=0}^{N-d-1} e^{j\left(\frac{\pi}{N}+\alpha\right)(n-p)} \\
B(\alpha)=\sum_{n, p=0}^{d-1} e^{-j\left(\frac{\pi}{N}+\alpha\right)(n-p)}=\sum_{n, p=0}^{d-1} e^{j\left(\frac{\pi}{N}+\alpha\right)(n-p)}
\end{gathered}
$$


To obtain (A.18) and (A.19), first the negative sign before $j$ is dissolved to $(n-p)$ to make $(p-n)$; then, using a simple change of indexes in summation (see (A.17)), $(p-n)$ is converted to $(n-p)$. Also, using $\cos (x)=\cos (-x)$, the third term of $\left|\tilde{X}_{N}(\alpha)\right|^{2}$ can be written as follows.

$$
C(\alpha)=2 \sum_{n=0}^{N-d-1} \sum_{p=N-d}^{N-1} \cos (\Theta(n, p)+\alpha(n-p))
$$

Taking (A.18)-(A.20) into account, $\left|\tilde{X}_{0}(\alpha)\right|^{2}$ and $\left|\tilde{X}_{N}(\alpha)\right|^{2}$ can be written based on $A(\alpha), B(\alpha)$ and $C(\alpha)$.

$$
\begin{gathered}
\left|\tilde{X}_{0}(\alpha)\right|^{2}=A(-\alpha)+B(\alpha)+C(-\alpha) \\
\left|\tilde{X}_{N}(\alpha)\right|^{2}=A(\alpha)+B(-\alpha)+C(\alpha)
\end{gathered}
$$

This simplified notation of $\left|X_{0}(\alpha)\right|^{2}$ and $\left|X_{N}(\alpha)\right|^{2}$ shapes the basis of the final stage of our proof.

\section{A.3 Proving $\alpha=0$ Is Maximum}

For a function $f(x)$ to have a maximum at $x_{0}$, its first and second derivatives at $x_{0}$ must be zero and negative, respectively. Hence, in the following we prove:

»Statement I: $\left.\frac{d}{d \alpha} \tilde{\mathscr{X}}(\alpha)\right|_{\alpha=0}=0$

» Statement II: $\left.\frac{d^{2}}{d \alpha^{2}} \tilde{X}(\alpha)\right|_{\alpha=0}<0$

From (A.21) and (A.22), $\left|\tilde{X}_{0}(\alpha)\right|^{2}$ and $\left|\tilde{X}_{N}(\alpha)\right|^{2}$ can be written as $F(\alpha)$ and $F(-\alpha)$, respectively. Besides, $\tilde{G}_{0}(\alpha)$ can be written as $F(\alpha)+F(-\alpha)$. It is known from the chain rule of derivative that $\frac{d}{d x} f(g(x))=g^{\prime}(x) f^{\prime}(g(x))$. Thus, $\frac{d}{d \alpha} F(-\alpha)=$ $-F^{\prime}(-\alpha)$. So the first derivative of $\tilde{G}_{0}(\alpha)$ is as follows.

$$
\frac{d}{d \alpha} \tilde{G}_{0}(\alpha)=F^{\prime}(\alpha)-F^{\prime}(-\alpha)
$$

For $\alpha=0$ the right side of (A.23) is zero. Notice that all derivations so far are based on a general case of $d$, and, as mentioned earlier, the same procedure can be followed if the $i=0$ window starts from an Even symbol. The main reason that allows this is the frequency separation which is equal to the symbol rate. This means the result of (A.23) can be generalized to any $\tilde{G}_{m}(\alpha)$. Considering the definition of $\tilde{\mathscr{X}}(\alpha)$ from (A.12), statement I is proved as follows: 


$$
\left.\frac{d}{d \alpha} \tilde{X}(\alpha)\right|_{\alpha=0}=\left.\sum_{m=0}^{N-1} \frac{d}{d \alpha} \tilde{G}_{m}(\alpha)\right|_{\alpha=0}=0
$$

Statement I shows that $\alpha=0$ is an extremum (maximum or minimum) for $\tilde{\mathscr{X}}(\alpha)$. To further prove that $\alpha=0$ is a maximum (and not a minimum), it must be shown that its second derivative is negative. Taking another derivative from both sides of (A.23) according to the chain rule, the $\frac{d^{2}}{d \alpha^{2}} \tilde{G}_{0}(\alpha)$ is derived as follows.

$$
\frac{d^{2}}{d \alpha^{2}} \tilde{G}_{0}(\alpha)=F^{\prime \prime}(\alpha)+F^{\prime \prime}(-\alpha)
$$

Moreover, for any $d,\left|\tilde{X}_{0}(\alpha)\right|^{2}=F(\alpha)$ and $\left|\tilde{X}_{N}(\alpha)\right|^{2}=F(-\alpha)$ have a maximum in $-\pi / N \leq \alpha \leq \pi / N$ (see the spectra between $f_{0}$ and $f_{1}$ in Fig. 5.2) which means both have a negative second derivative in this interval including $\alpha=0$. As a result, $\frac{d^{2}}{d \alpha^{2}} \tilde{G}_{m}(\alpha)$ is negative at $\alpha=0$ so statement II holds as follows.

$$
\left.\frac{d^{2}}{d \alpha^{2}} \tilde{\mathscr{X}}(\alpha)\right|_{\alpha=0}=\left.\sum_{m=0}^{N-1} \frac{d^{2}}{d \alpha^{2}} \tilde{G}_{m}(\alpha)\right|_{\alpha=0}<0
$$

From statements I and II, it is concluded that the sum in (A.2) has a maximum at $\alpha=0$ or $\Omega=\omega_{c} T_{s}$. 


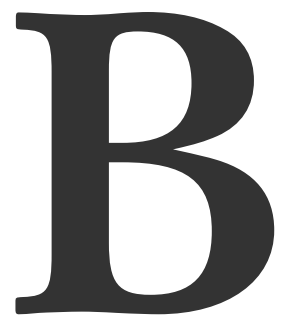

\section{Complexity OF Hara SYNCHRONIZATION WITH SFFT}

In the conventional synchronization algorithm NI-point FFTs are used. As mentioned in Chapter 5, an SFFT structure similar to [30] is the best option for the conventional algorithm. However, the zero-padding in our application allows us to devise a simpler version of the SFFT in [30]. Due to zero-padding, in the first few stages of the FFT some butterflies have zero inputs and the others have only one non-zero input which means they simply need to pass this nonzero input without any operations. Here, these stages are called pruned stages. A similar simplification to the FFT structure (and not SFFT) was introduced in [65].

To clarify, let us take a look at a 16-point FFT architecture where $N=4$ and $I=4$. Figure B.1 shows a pruned architecture of this FFT which is the modified version of the SFFT in [30]. The black nodes which are not connected to any edges are zeros. When the sample $x(n)$ is added, only high-lighted butterflies and the data over solid black lines need to be calculated (or passed through) and the rest of the nodes and the dotted grey lines may use previously calculated values. Notice that in the first and the second stages (pruned stages), butterflies only pass through the input. That is why the number of multiplications for the SFFT in case of zero-padding will be less than what is reported in [30].

When a zero-padding factor of $I$ is utilized, there are $\log _{2} I$ pruned stages which do not need any calculation whereas they are counted in the complexity analysis of [30]. To have a correct complexity analysis for the conventional algorithm, the computations in pruned stages must be reduced from the overall complexity of the SFFT. As shown in [30], for the $s^{t h}$ stage $\left(s=1, \ldots, \log _{2} N I\right)$ of the Radix$2 N I$-point FFT structure, there are $2^{s-1}$ groups of butterflies. A group of butterflies consists of those butterflies which use the same twiddle factor and can be seen in the figure as a set of circles close to each other. Each group is shown with a dashed circle in Figure B.1. From each group, one butterfly needs to be calculated in each update of the window, so the number of butterflies required 


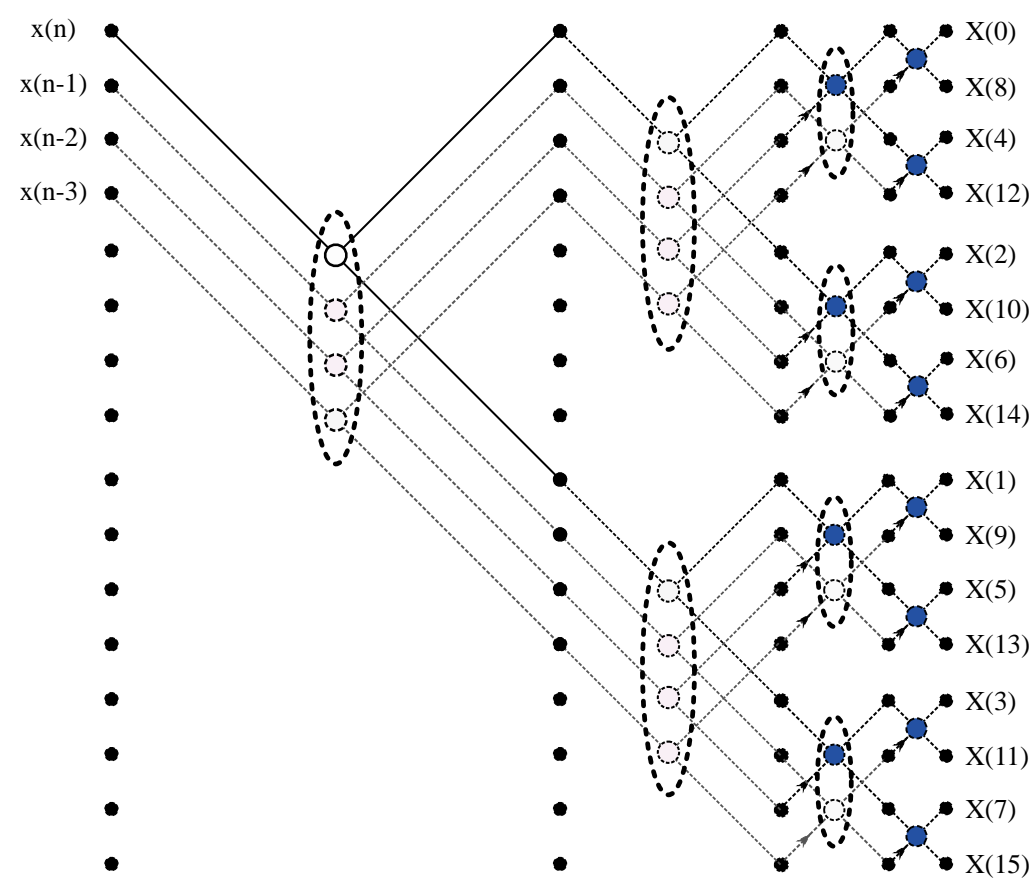

Figure B.1: The pruned sliding FFT

for each update in the $N I$-point $\operatorname{SFFT}\left(B F_{S F F T}\right)$ is:

$$
B F_{S F F T}=\sum_{s=1}^{\log _{2}(N I)} 2^{s-1}=N I-1
$$

In case of zero-padding, the first $\log _{2} I$ stages do not need computation (they just pass through the inputs); thus, the number of butterflies which must be calculated in each update of the SFFT with zero-padding is:

$$
B F_{S F F T}^{\text {Pruned }}=\sum_{s=1}^{\log _{2}(N I)} 2^{s-1}-\sum_{s=1}^{\log _{2}(I)} 2^{s-1}=N I-I
$$

Starting from the initial state of zero, the SFFT needs $N$ updates at the beginning so that the FFT for the first set of $N$ samples is ready. Notice that the samples are sent into the SFFT one by one and it takes $N$ updates to have all samples of the first window as inputs. Afterwards, considering $L$ as the length of the preamble, $N(L-1)$ updates are required for sliding the window over the whole preamble ${ }^{1}$ making a total of $N L$ updates.

\footnotetext{
${ }^{1} N$ delays for each symbol, from symbol 1 to symbol $L-1$ of the preamble.
} 
Each butterfly is composed of one complex multiplication and two complex additions. So the number of complex multiplications and additions for calculating the SFFT are as follows.

$$
\begin{gathered}
C M_{S F F T}=N L \times B F_{S F F T}^{\text {Pruned }} \\
C A_{S F F T}=2 \times N L \times B F_{S F F T}^{\text {Pruned }}
\end{gathered}
$$

Now that the required number of operations are calculated for each part of synchronization, memory usage is calculated in the following.

In case of the SFFT, for the outputs of each butterfly (two outputs) in the FFT which is not calculated during an SFFT update, two memories are needed to store their values from the previous window [30]. As discussed earlier, for all first $\log _{2} I$ stages of the pruned SFFT only $N-1$ previous input samples must be stored as these stages only pass through input samples. For the other $\log _{2} N$ stages, there are $N I / 2$ butterflies per stage and $(N I / 2) \log _{2} N$ butterflies in total. Among these only $I(N-1)$ butterflies are calculated according to above discussion and the rest use the stored value from the previous window calculations; thus, the required memory for calculation of SFFT is equal to:

$$
M_{\text {Calc }}^{S F F T}=2 \times\left[(N-1)+2 \times\left((N I / 2) \log _{2} N-I(N-1)\right)\right],
$$

where the first term in the right side of the equation is for storing the previous samples and the second one is the required memory for butterfly outputs. The factor two before the brackets in (B.5) is the result of the fact that the values are complex and need two memories for storage. Besides, the factor two in the second term inside the bracket shows the number of outputs for each butterfly.

To compute the memory used for the accumulation of DFT bin values, $M_{A c c}^{S F F T}$, it should be noticed that for each delay value two sets of memories are used to store the accumulated DFT bins magnitudes; odd and even symbols. For the conventional algorithm, each DFT has NI bins. So, considering $N$ delay values we have:

$$
M_{A c c}^{S F F T}=2 I N^{2}
$$




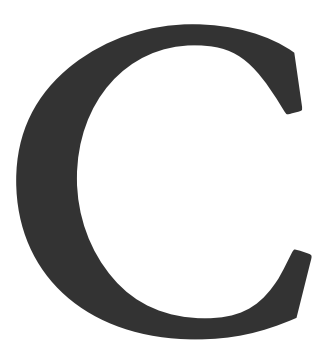

\section{Hybrid Demodulator in Time-Varying Channel}

In this appendix the detailed expressions for $F_{0}$ and $F_{1}$ are derived. Substituting $Y_{k}$ from (6.25) to (6.27) and (6.28) we have:

$$
\begin{aligned}
F_{0}= & \sum_{k=1}^{4} a^{2(k+1)}|h|^{2} X_{k} \\
& +\sum_{k=1}^{4}\left(a^{k+3} \sum_{i=1}^{k-1} a^{i-1} \sqrt{1-a^{2}} b \eta_{k-i}^{*} X_{k}\right) \\
& +\sum_{k=1}^{4}\left(a^{k-1} \sum_{i=1}^{k+3} a^{i-1} \sqrt{1-a^{2}} b^{*} \eta_{k+4-i} X_{k}\right) \\
F_{1}= & \sum_{k=1}^{4} a^{2(k+1)}|h|^{2} X_{k} e^{-j \omega_{s e p} k T_{s}} \\
& +\sum_{k=1}^{4} e^{-j \omega_{s e p} k T_{s}}\left(a^{k+3} \sum_{i=1}^{k-1} a^{i-1} \sqrt{1-a^{2}} b \eta_{k-i}^{*} X_{k}\right. \\
& \left.+a^{k-1} \sum_{i=1}^{k+3} a^{i-1} \sqrt{1-a^{2}} b^{*} \eta_{k+4-i} X_{k}\right)
\end{aligned}
$$

From (6.6) and considering $E_{S} / N=1$ for easier notations (this assumption does not affect generality as this factor is present in both distortion and signal components), we can write $X_{k}=\exp \left(j\left(\Delta \omega_{n} k T_{s}+\Delta \varphi_{n}+\theta\right)\right)$ where all phase components in $X_{k}$ other than information bearing phase, $\Delta \varphi_{n}$, are absorbed in $\theta$. Since this extra phase component is removed at the second stage and does not affect our calculations here, we may ignore that to simplify notations. Now, in case $\Delta \omega_{n}=0, X_{k}$ can be taken out of the summation in (C.1) to achieve: 


$$
\begin{aligned}
F_{0}= & e^{j \Delta \varphi_{n}} \sum_{k=1}^{4} a^{2(k+1)}|h|^{2} \\
& +e^{j \Delta \varphi_{n}} \sum_{k=1}^{4}\left(a^{k+3} \sum_{i=1}^{k-1} a^{i-1} \sqrt{1-a^{2}} b \eta_{k-i}^{*}\right) \\
& +e^{j \Delta \varphi_{n}} \sum_{k=1}^{4}\left(a^{k-1} \sum_{i=1}^{k+3} a^{i-1} \sqrt{1-a^{2}} b^{*} \eta_{k+4-i}\right)
\end{aligned}
$$

For $N=4$, since $\omega_{\text {sep }}=2 \pi / T$, we have $e^{-j \omega_{\text {sep }} k T_{s}}=(-j)^{k}$. Thus, $F_{1}$ is derived as follow.

$$
\begin{aligned}
F_{1}= & e^{j \Delta \varphi_{n}} \sum_{k=1}^{4} a^{2(k+1)}|h|^{2}(-j)^{k} \\
& +e^{j \Delta \varphi_{n}} \sum_{k=1}^{4}\left((-j)^{k} a^{k+3} \sum_{i=1}^{k-1} a^{i-1} \sqrt{1-a^{2}} h \eta_{k-i}^{*}\right. \\
& \left.+(-j)^{k} a^{k-1} \sum_{i=1}^{k+3} a^{i-1} \sqrt{1-a^{2}} b^{*} \eta_{k+4-i}\right)
\end{aligned}
$$

Both $F_{0}$ and $F_{1}$ have a signal component (first term in both) and other terms resulting from distortion which is caused by the random variable $\eta_{i}$.

$\eta_{i} \sim \mathscr{C} \mathscr{N}\left(0, \sigma_{b}^{2}\right)$ and $h$ describes the channel state at the beginning of the $(n-$ $1)^{t h}$ symbol. The effect of $h$ is the same for all samples during symbol detection. Moreover, only the magnitude of $b$ affects the signal component (the first term in (6.25)) and the phase component is removed by the autocorrelation stage. As a result, although strictly speaking $h \sim \mathscr{C} \mathscr{N}\left(1, \sigma_{b}^{2}\right)$, we can treat it as a deterministic variable when calculating the effect of distortion during these three symbols.

In the distortion terms of both $F_{0}$ and $F_{1}, e^{j \Delta \varphi_{n}}$ can be absorbed in the random variable and since its magnitude is one it does not change the variance. Moreover, $\sqrt{1-a^{2}} b^{*} \eta_{i}=\left(\sqrt{\left(1-a^{2}\right)} b \eta_{i}^{*}\right)^{*}$ is denoted as a random variable $\zeta_{i} \sim \mathscr{C} \mathscr{N}\left(0,\left(1-a^{2}\right)|b|^{2} \sigma_{h}^{2}\right)$. Using these, after some mathematical manipulation we have: 


$$
\begin{gathered}
F_{0}=e^{j \Delta \varphi_{n}} \sum_{k=1}^{4} a^{2(k+1)}|h|^{2}+\sum_{m=1}^{4} a^{2-m} \sum_{k=1}^{4} a^{2 k} \zeta_{m} \\
+\sum_{m=5}^{7} a^{m-6} \sum_{k=1}^{8-m} a^{2 k} \zeta_{m} \\
+\sum_{m=1}^{3} a^{2-m} \sum_{k=m+1}^{4} a^{2 k} \zeta_{m}^{*} \\
F_{+1} \approx \sum_{m=1}^{4} a^{2-m} \sum_{k=1}^{4}(-j)^{k} a^{2 k} \zeta_{m} \\
+\sum_{m=5}^{7} a^{m-6} \sum_{k=1}^{8-m}(-j)^{k+1} a^{2 k} \zeta_{m} \\
+\sum_{m=1}^{3} a^{2-m} \sum_{k=m+1}^{4}(-j)^{k} a^{2 k} \zeta_{m}^{*}
\end{gathered}
$$

In (C.6), the term with the signal component is almost zero due to $(-j)^{k}$ factor. It is not exactly zero (thus the sign $\approx$ ) but it is negligible compared to the signal component in $F_{0}$ and noise components in $F_{1}$. For other values of $N$, the same conclusion can be achieved. The same factor $(-j)^{k}$ is the reason for lower power of noise in $F_{1}$.

Each component including $\zeta_{m}$ is a circularly symmetric Gaussian variable. The sum of circularly symmetric Gaussian variables is a circularly symmetric variable as well with a variance equal to the sum of the variances; however, this is not exactly true if $\zeta_{m}$ is added to its conjugate $\zeta_{m}^{*}$. In (C.5) and (C.6), for $m=1,2,3$, components with $\zeta_{m}$ are added to components which include $\zeta_{m}^{*}$. As a result, the obtained random variable is not precisely a circularly symmetric variable i.e. the variance of its real and imaginary parts are slightly different; nevertheless, the variance of the resulting random variable is still the sum of the variances of $\zeta_{m}$ and $\zeta_{m}^{*}$. For simpler calculations, we approximate this sum of random variables with a circularly symmetric Gaussian random variable with the same variance. Thus, merging the last three terms (including $\zeta_{m}$ and $\zeta_{m}^{*}$ ) of $F_{0}$ and all terms of $F_{1}, F_{0}$ and $F_{1}$ can be written in the form of:

$$
\begin{gathered}
F_{0}=\sqrt{P_{S}} e^{-j \Delta \varphi_{n}}+\sqrt{P_{N, 0}} \eta_{0}^{T} \\
F_{+1} \approx \sqrt{P_{N,+1}} \eta_{1}^{T}
\end{gathered}
$$

where: 


$$
P_{S}=S|b|^{4}
$$

$$
P_{N, 0}=\Upsilon_{0}|b|^{2} \sigma_{b}^{2}\left(1-a^{2}\right)
$$

$$
P_{N,+1}=\Upsilon_{+1}|b|^{2} \sigma_{b}^{2}\left(1-a^{2}\right)
$$

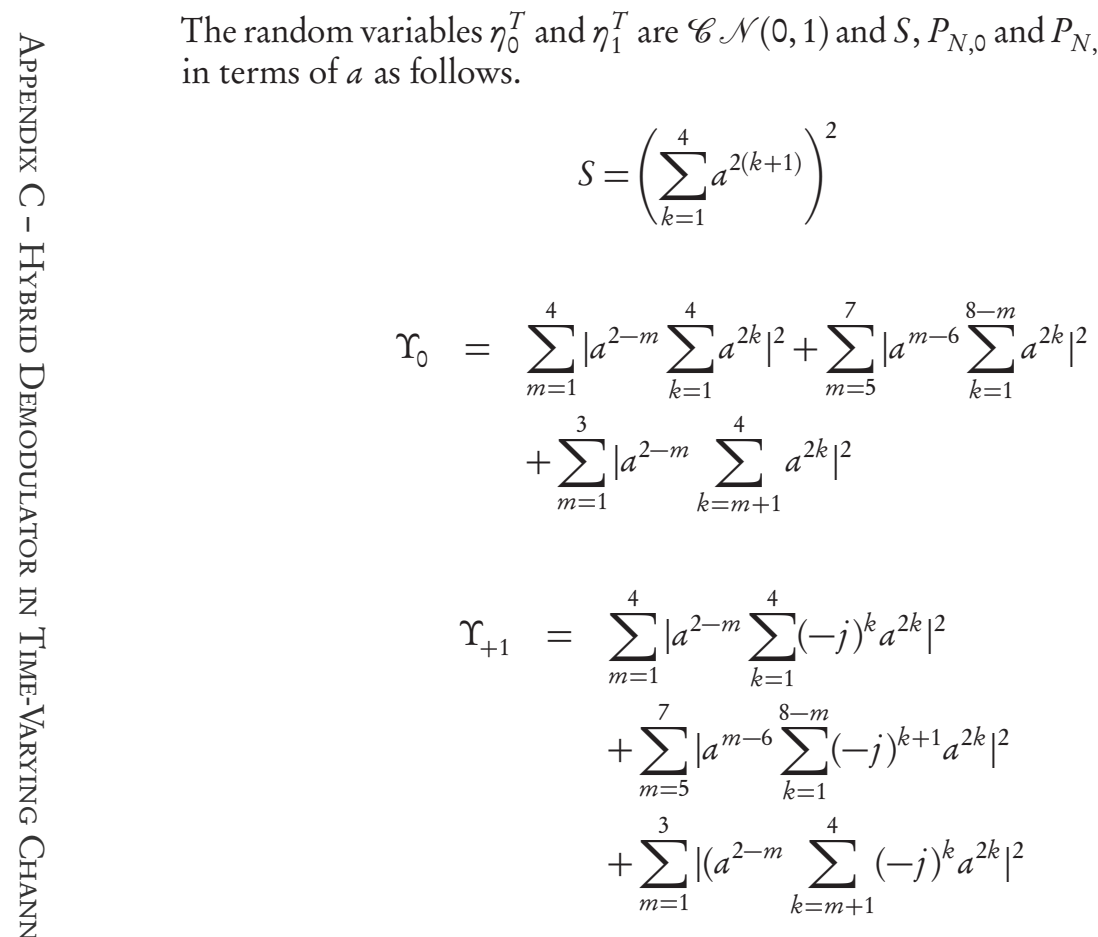




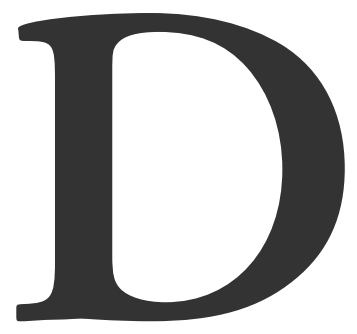

\section{BER Curves FOR Hybrid Demodulator}

In this chapter the detailed BER curves for the scenarios in Chapter 6 are presented and discussed.

\section{D.1 BeR Curves for Constant Symbol Rate Scenario}

For the sake of clarity, different combinations are compared in two cases. In Case I, all combinations which can obtain repetition orders ( $\mathrm{RO})$ of two and three in Table 6.3 are compared. Case II considers the combinations which achieve repetition orders of 4 and 5 . The diagrams for Case I also include 2PSK and 2FSK (shown by $2 \mathrm{P}$ and $2 \mathrm{~F}$, respectively). Besides, Case II diagrams also include the BER curves for DD8PSK (shown by $8 \mathrm{P}$ ) as a reference for comparison.

The BER performance of the system in a Rayleigh fading channel for three different maximum Doppler shift values is demonstrated in Figure D.1-Figure D.3. The graphs on the top and the bottom show Case I and Case II, respectively. In each graph, similar line patterns (solid, dotted and dash-dotted) correspond to the same RO.

As expected, for a time-invariant channel in Figure D.1a and Figure D.1b, 2P is the best as higher orders and hybrid combinations do not add the diversity but only degrade performance. As can be seen, adding FSK to PSK modulation in Rayleigh channel does not lead to considerable improvement. This is in agreement with the conclusions in [35]. In all modulations, the most determinant factor for the BER performance is the order of MPSK modulation.

Figure D.2a and Figure D.2b show the BER curves in presence of a time-varying channel with a Jakes spectrum and a maximum Doppler shift of $2 \mathrm{~Hz}$. For $R O>2$ (solid lines in Case I and all curves in Case II), using hybrid modulation can decrease the required $E_{b}^{\operatorname{Inf}} / N_{0}$ for BER $=0.1 \%$ by 5 to almost $11 \mathrm{~dB}$ compared to MPSK with the same RO. For a certain $R O$, increasing the FSK 


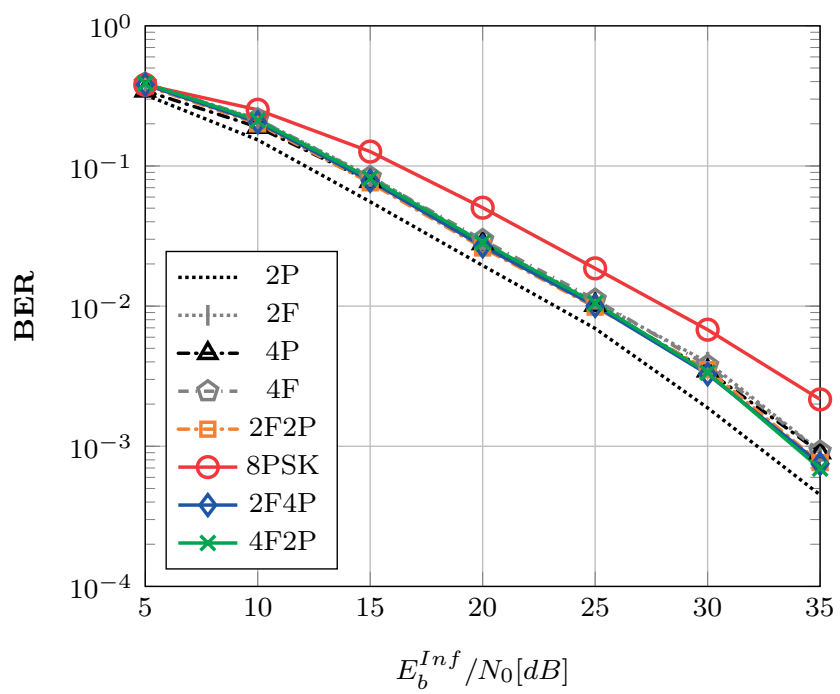

(a)

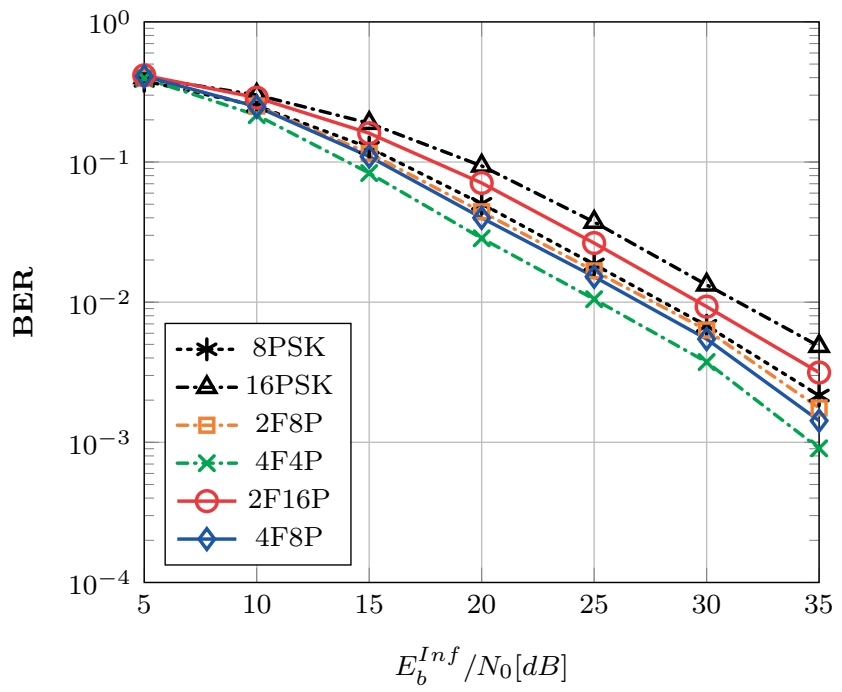

(b)

Figure D.1: BER curves for the system of Figure 6.4 and constant symbol rate scenario. The channel model is Rayleigh with $D_{\max }=0$. (a) and (b) show Case I and Case II, respectively. Each line pattern (solid and dashdotted) belongs to the same repetition order. 
order makes it possible to use lower order DDMPSK. The improvement in the BER performance mostly depends on the variation in DDMPSK order. As can be seen in Figure 6.5a and Figure 6.5 (the BER curves for AWGN channel in Chapter 6), the $E_{b}^{\text {Inf }} / N_{0}$ needed for $4 \mathrm{PSK}$ to achieve $\mathrm{BER}=0.1 \%$ is $4 \mathrm{~dB}$ less than what needed for 8PSK. The same behavior can be seen in Figure D.2. In Case I and $R O=3$ (solid lines), going from $8 \mathrm{PSK}$ to $2 \mathrm{~F} 4 \mathrm{P}$ can save more than $5 \mathrm{~dB}$ at $\mathrm{BER}=0.1 \%$ while from $2 \mathrm{~F} 4 \mathrm{P}$ to $4 \mathrm{~F} 2 \mathrm{P}$ an improvement less than $1 \mathrm{~dB}$ is achieved. On the other hand, for $R O=4,2 \mathrm{~F} 8 \mathrm{P}$ achieves more than $6 \mathrm{~dB}$ improvement compared to 16PSK and using 4F4P leads to a $5 \mathrm{~dB}$ improvement compared to $2 \mathrm{~F} 8 \mathrm{P}$ (and more than $11 \mathrm{~dB}$ compared to $16 \mathrm{PSK}$ ). $4 \mathrm{~F}$ is a little bit better than $4 P$ at $\mathrm{BER}=0.1 \%$ and has a lower error floor; nevertheless, similar performance is achieved using $2 \mathrm{~F} 2 \mathrm{P}$ and about $4 \mathrm{~dB}$ energy is saved (at $B E R=0.1 \%$ ) if $4 \mathrm{FSK}$ is combined with 2PSK.

In Figure D.3a and Figure D. 3 b, the simulation results for $D_{\max }=5 \mathrm{~Hz}$ are presented. In these simulations, the effect of channel time-selectivity is more severe than $D_{\max }=2 \mathrm{~Hz}$. The same trends as in the graphs for $D_{\max }=2 \mathrm{~Hz}$ can also be detected for these diagrams. The main difference is that the error floor in these cases is higher than those in case $D_{\max }=2 \mathrm{~Hz}$.

For each repetition order, the higher the order of LFSK in hybrid modulation the lower will be the error floor. In case of $R O=3$ and $R O=4$, the error floor decreases by almost two orders of magnitude if 8PSK and 16PSK are changed to $4 \mathrm{~F} 2 \mathrm{P}$ and 4F4P, respectively. Due to higher error floors caused by $D_{\max }=5$ $\mathrm{Hz}$, which decreases the slope of the BER curves, the energy saving achieved by hybrid modulation compared to DDMPSK at $\mathrm{BER}=0.1 \%$ increases up to $11 \mathrm{~dB}$ for $R O=3$ and more than $17 \mathrm{~dB}$ for $R O=4$. The effect of higher order PSK on the increase of the error floor can be observed when comparing 4F2P and 4F4P combinations. $4 \mathrm{~F} 4 \mathrm{P}$ provides a higher repetition order and that is why the slope of the BER curve is steeper than 4F2P (between 10 to $20 \mathrm{~dB}$ ); nevertheless, the error floor of $4 \mathrm{~F} 4 \mathrm{P}$ is slightly higher than the error floor of $4 \mathrm{~F} 2 \mathrm{P}$ which is due to the worse error floor of 4PSK compared to 2PSK.

For both $D_{\max }=2 \mathrm{~Hz}$ and $D_{\max }=5 \mathrm{~Hz}$, the best modulation is the $4 \mathrm{~F} 4 \mathrm{P}$ combination. Applying 4F4P modulation, the energy per information bit required to achieve $\mathrm{BER}=0.1 \%$, is only $5 \mathrm{~dB}$ higher compared to DDBPSK in AWGN channel. This is an improvement of almost $20 \mathrm{~dB}$ compared to DDBPSK without time diversity in case of $D_{\max }=2 \mathrm{~Hz}$. Notice that these results are achieved with repetition coding (the simplest coding technique). The error floor can be decreased and the performance can be further improved if error correction coding techniques are used. The perspective of using error correction codes is the main reason that we mostly focused on the $E_{b}^{I n f} / N_{0}$ required for $\mathrm{BER}=0.1 \%$.

Figure D.4 and Figure D.5 depict the BER curves for a Rician channel with $K=1$ and for $D_{\max }=2 \mathrm{~Hz}$ and $D_{\max }=5 \mathrm{~Hz}$, respectively. Observations similar to the Rayleigh channel cases can be made for the Rician channel. The better performance of hybrid modulation can be seen in the Rician channel 


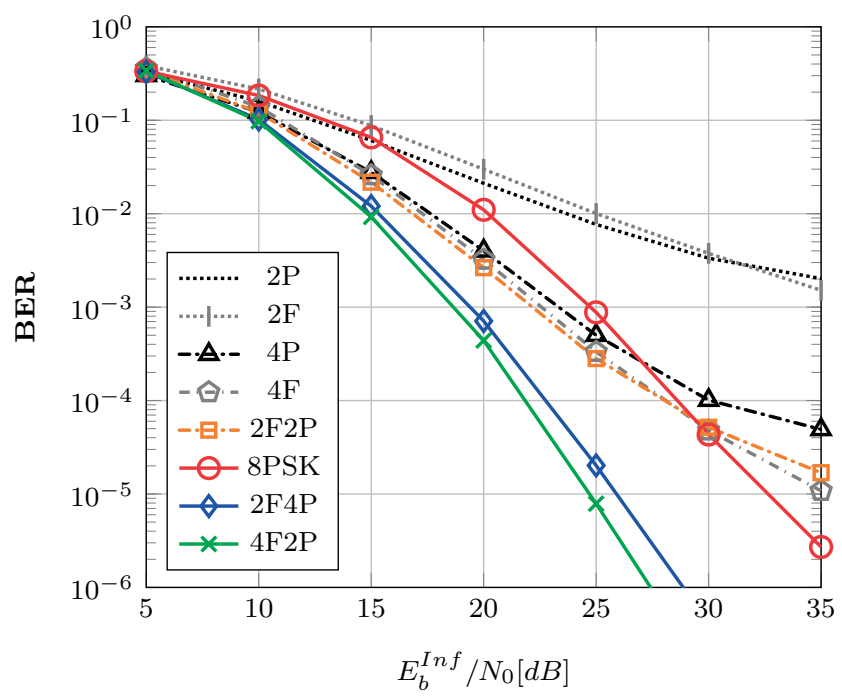

(a)

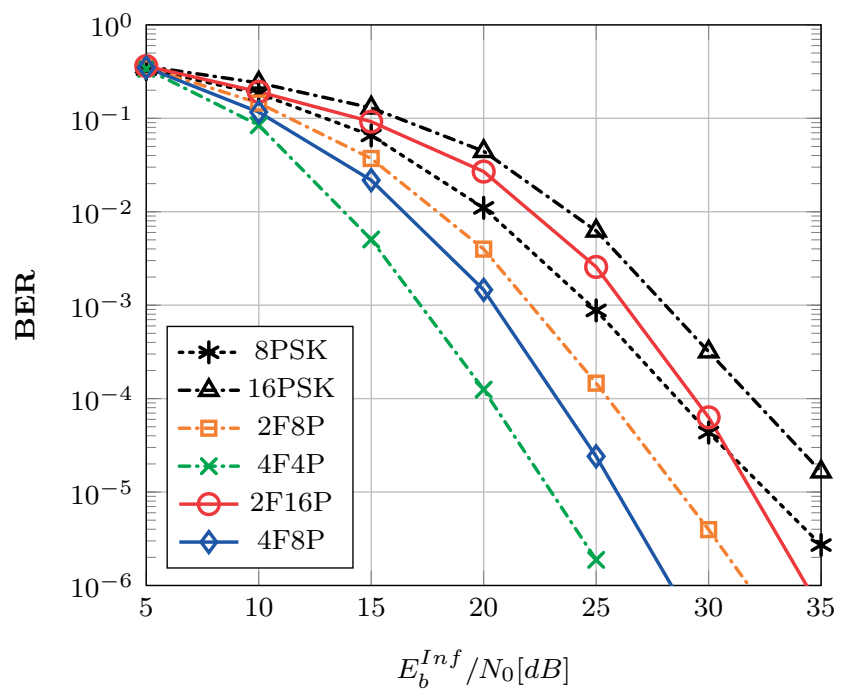

(b)

Figure D.2: BER curves for the system of Figure 6.4 and constant symbol rate scenario. The channel model is Rayleigh with $D_{\max }=2 \mathrm{~Hz}$ and Jakes Doppler spectrum. (a) and (b) show Case I and Case II, respectively. Each line pattern (solid and dashdotted) belong to the same repetition order. 


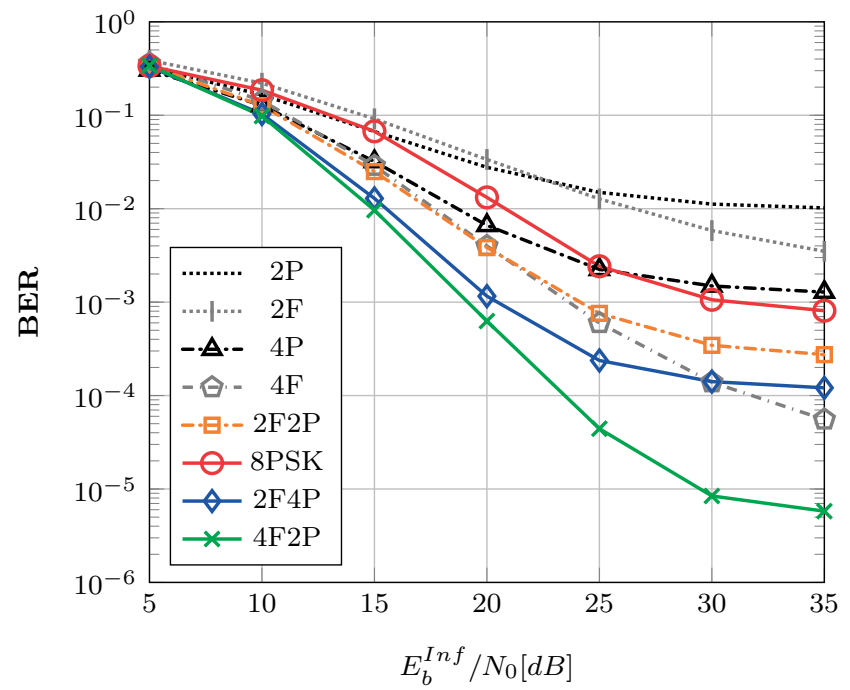

(a)

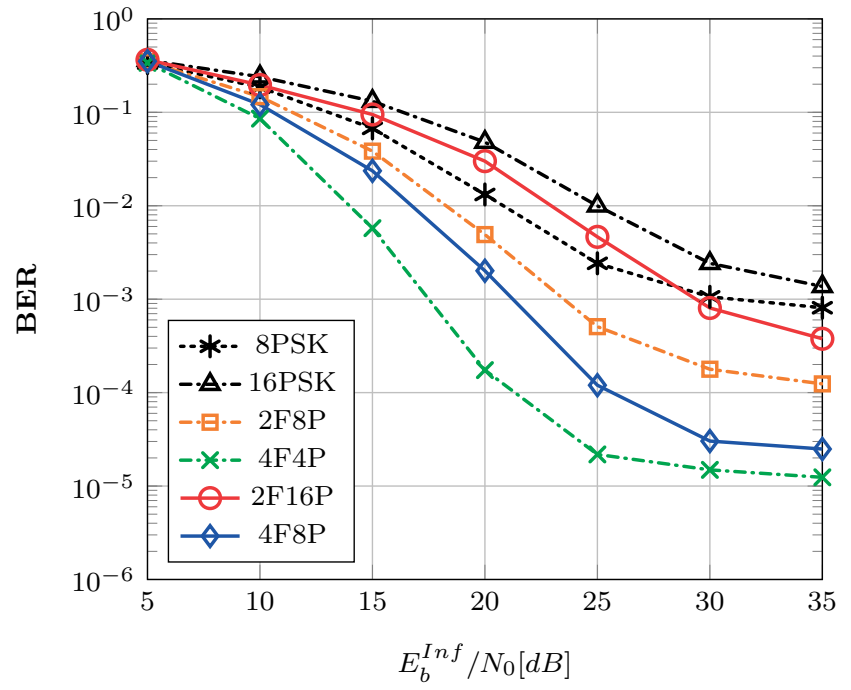

(b)

Figure D.3: BER curves for the system of Figure 6.4 and constant symbol rate scenario. The channel model is Rayleigh with $D_{\max }=5 \mathrm{~Hz}$ and Jakes Doppler spectrum. (a) and (b) show Case I and Case II, respectively. Each line pattern (solid and dashdotted) belongs to the same repetition order. 


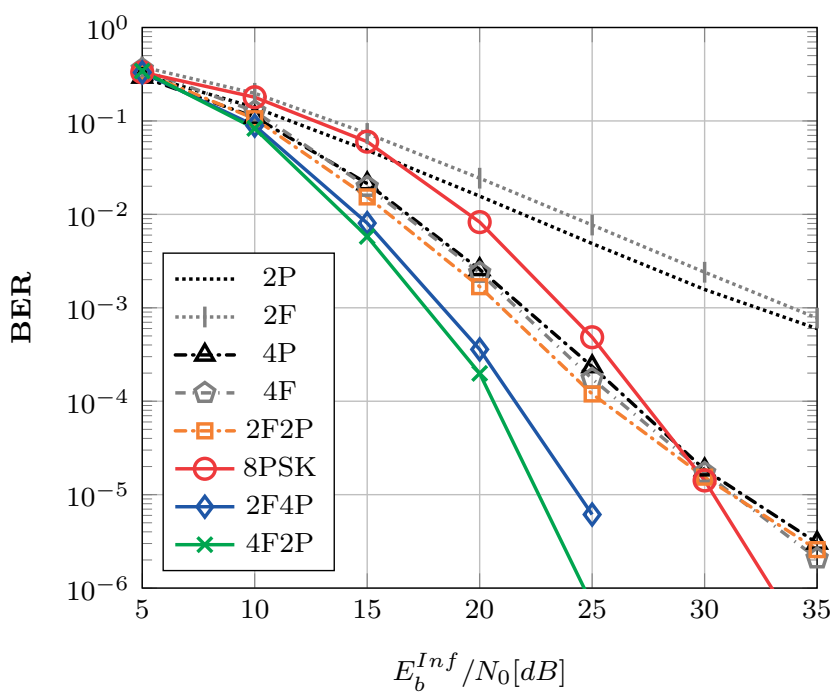

(a)

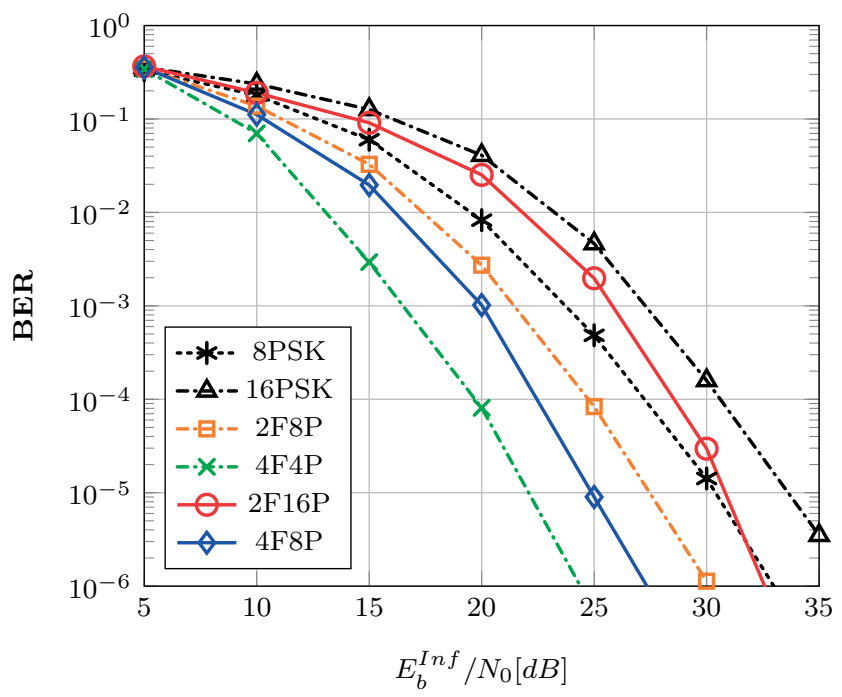

(b)

Figure D.4: BER curves for constant symbol rate scenario Case I (a) and Case II (b). The channel model is Rician $(K=1)$ with $D_{\max }=2 \mathrm{~Hz}$ and a rounded Doppler spectrum. 


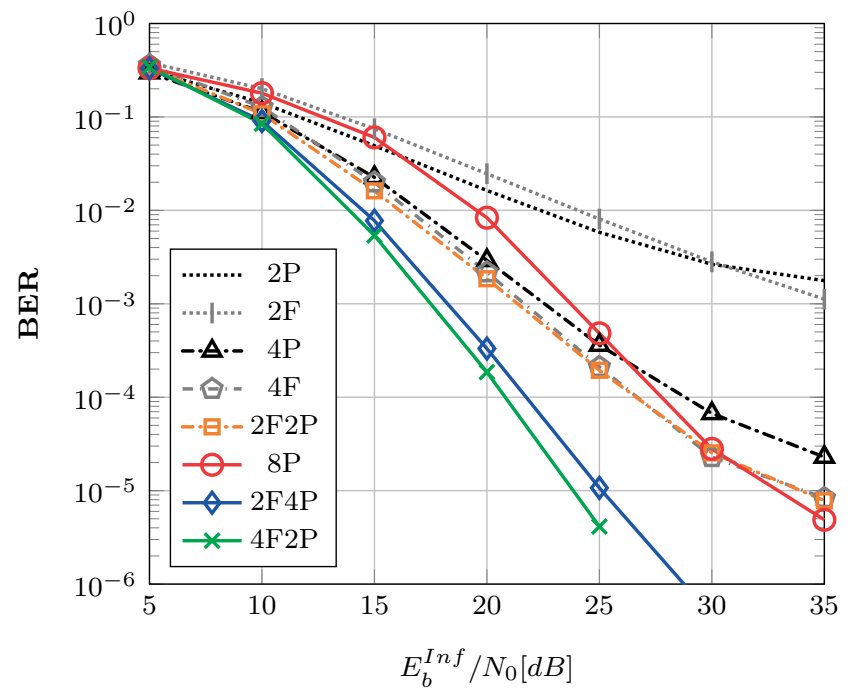

(a)

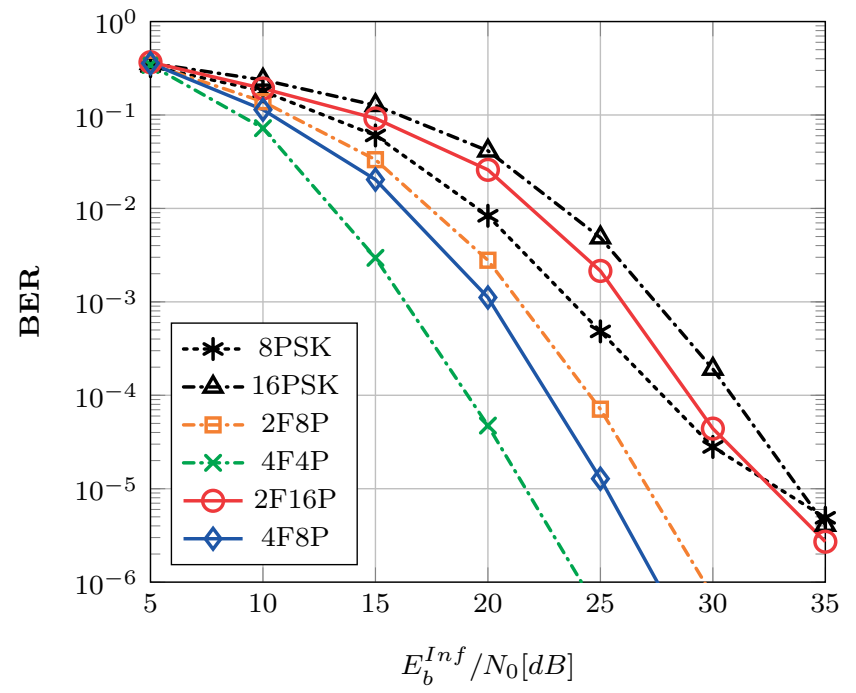

(b)

Figure D.5: BER curves for constant symbol rate scenario Case I (a) and Case II (b). The channel model is Rician $(K=1)$ with $D_{\max }=5 \mathrm{~Hz}$ and a rounded Doppler spectrum. 
as well. Similar to the Rayleigh channel results, the best combination is 4F4P modulation.

\section{D.2 BER Curves for Equal Bandwidth Scenario}

Two cases are investigated in this scenario. Case I and Case II which consider the two first columns of Table 6.4 and the last two columns of Table 6.4, respectively. All the combinations in each case have the same bandwidth $\left(3 R_{S}^{R e f}\right.$ for Case I and $5 R_{S}^{R e f}$ for Case II) while providing various repetition orders.

The BER curves for the equal bandwidth scenario in a Rayleigh fading channel with $D_{\max }=0$ (constant channel) are shown in Figure D.6a and Figure D.6b. Since no diversity is added by increasing the raw bit-rate, a significant improvement is not expected for hybrid modulation. Similar to the constant symbol rate scenario, the performance of hybrid modulation schemes including 2PSK and 4PSK in a constant channel are very close while increasing the modulation order to eight causes BER performance loss. The 2PSK (with increased symbol rate) has almost the same performance as the hybrid methods.

Figure D.7a and Figure D. $7 \mathrm{~b}$ demonstrate the BER curves for $D_{\max }=2 \mathrm{~Hz}$ and the equal bandwidth scenario. For the lowest $R O$ in both Case I and Case II (curves with triangle marker), hybrid modulation can save considerable energy $\left(E_{b}^{I n f} / N_{0}\right)$ compared to DDMPSK; more than $10 \mathrm{~dB}$ in Case I and almost $5 \mathrm{~dB}$ in Case II at $\mathrm{BER}=0.1 \%$. This significant difference is observed since the $R O$ for 2PSK with a higher symbol rate (1.5 and 2.5 when the symbol rate is $1.5 R_{S}^{R e f}$ and $2.5 R_{S}^{R e f}$, respectively) is less than the ROs achieved by hybrid modulation which are 2 and 3 for 2F2P and 4F2P, respectively (see Table 6.4). For 8PSK (the curves with square markers), the hybrid modulation performs slightly better than faster $8 \mathrm{PSK}$ at $\mathrm{BER}=0.1 \%$ despite the fact that fast $8 \mathrm{PSK}$ achieves a higher repetition order $(\mathrm{RO})$ in both Case I and Case II. The difference between hybrid modulation and higher symbol rate 8PSK decreases when the $E_{b}^{I n f}$ increases (BER decreases). As can be seen the higher symbol rate MPSK crosses the hybrid modulation curve at BER $=10^{-6}$ for Case I and $B E R<10^{-6}$ for Case II.

The BER performance for $D_{\max }=5 \mathrm{~Hz}$ is shown in Figure D.8a and Figure D.8b. The same trends as explained for $D_{\max }=2 \mathrm{~Hz}$ are observed here as well. The effect of increasing $D_{\max }$ on the error floor of hybrid modulation is worse than its impact on higher symbol rate MPSK. As a consequence, the error floor of hybrid modulation increases more than the error floor of fast DDMPSK which has a higher symbol rate. This brings the crossing point of hybrid modulation and high symbol rate DDMPSK to higher than $10^{-6}$ which was the case in $D_{\max }=2 \mathrm{~Hz}$. At a $\mathrm{BER}=0.1 \%$, the $4 F 4 P$ requires the lowest $E_{b}^{I n f} / N_{0}$ values which is $2 \mathrm{~dB}$ less than the best DDMSPK (4PSK-2.5 $R_{S}$ ).

The BER curves for a Rician channel with $K=1$ and for $D_{\max }=2 \mathrm{~Hz}$ and 


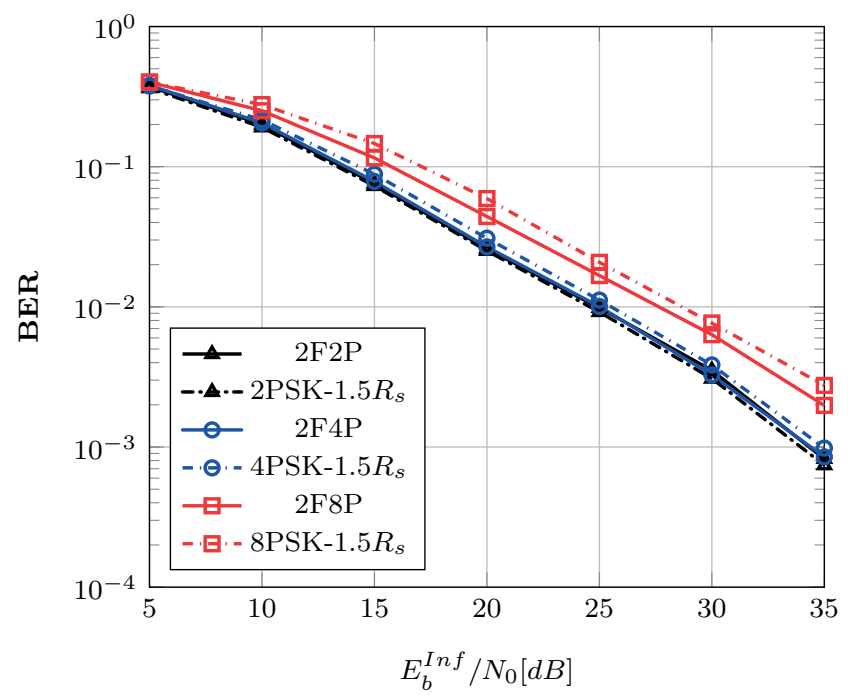

(a)

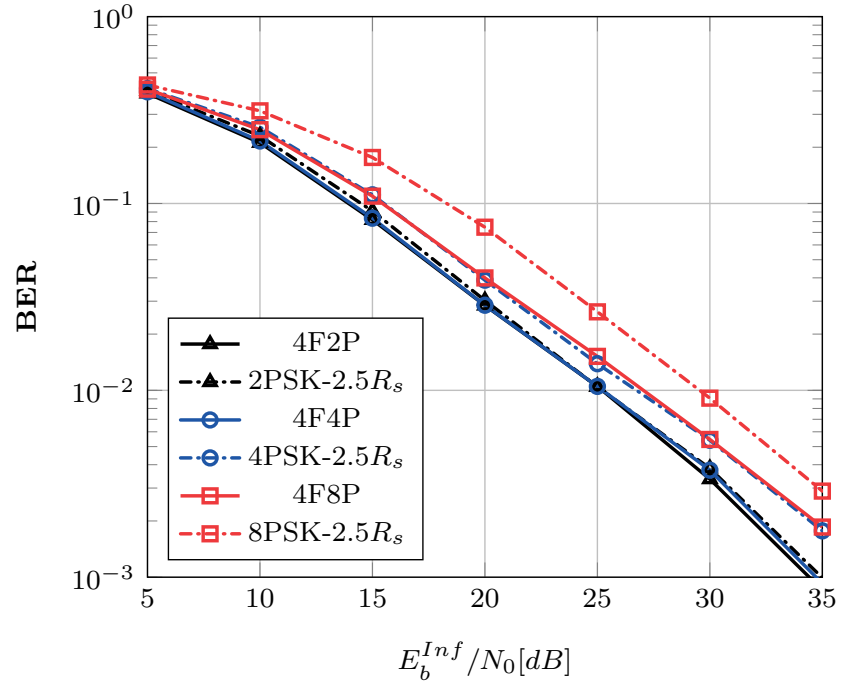

(b)

Figure D.6: BER curves for the system of Figure 6.4 and equal bandwidth scenario. The channel model is Rayleigh with $D_{\max }=0$ and Jakes Doppler spectrum. (a) and (b) show Case I and Case II, respectively. Each marker/color corresponds to one PSK order; triangle (black), circle (blue) and square (red) show 2PSK, 4PSK and 8PSK, respectively. For each color/marker, the solid lines show the hybrid modulation and the dash-dotted lines show the corresponding MPSK modulation with increased symbol rate. 


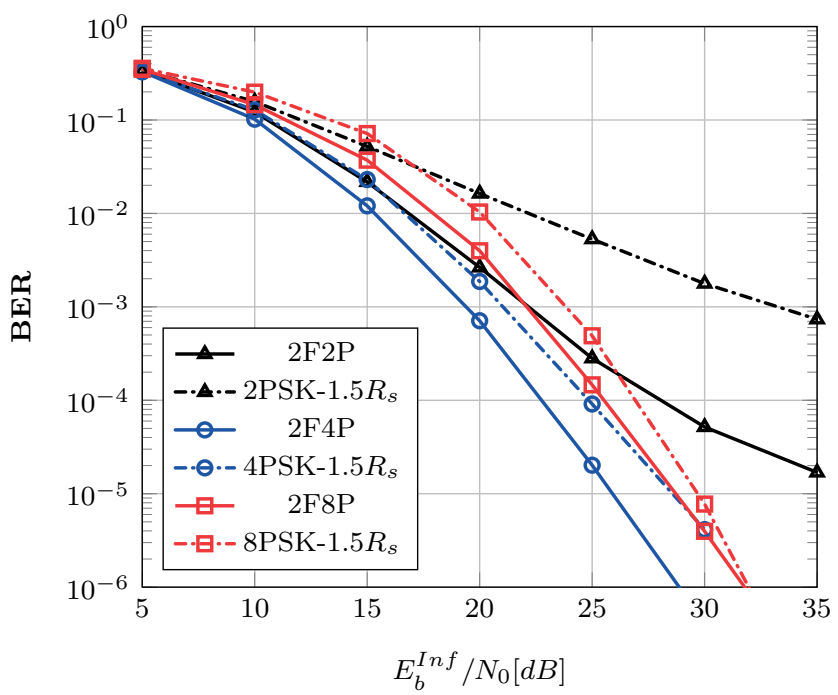

(a)

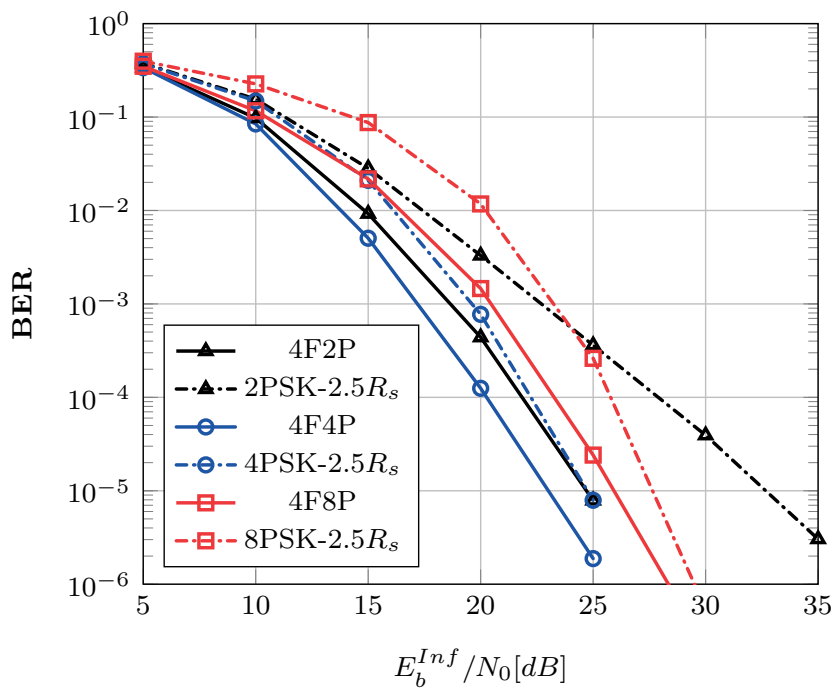

(b)

Figure D.7: BER curves for the system of Figure 6.4 and equal bandwidth scenario. The channel model is Rayleigh with $D_{\max }=2 \mathrm{~Hz}$ and Jakes Doppler spectrum. (a) and (b) show Case I and Case II, respectively. Each marker/color corresponds to one PSK order; triangle (black), circle (blue) and square (red) show 2PSK, 4PSK and 8PSK, respectively. For each color/marker, the solid lines show the hybrid modulation and the dash-dotted lines show the corresponding DDMPSK modulation with increased symbol rate. 


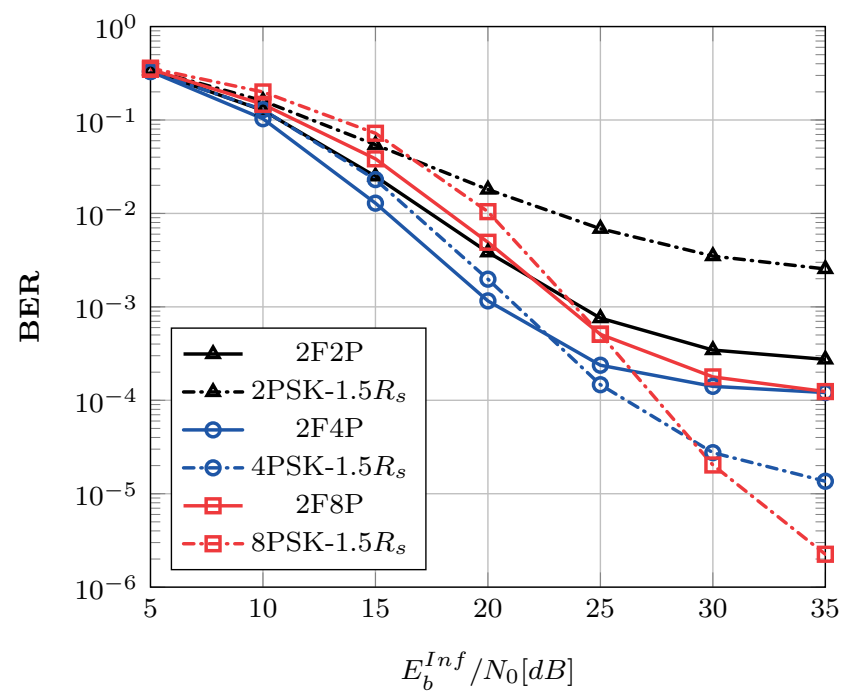

(a)

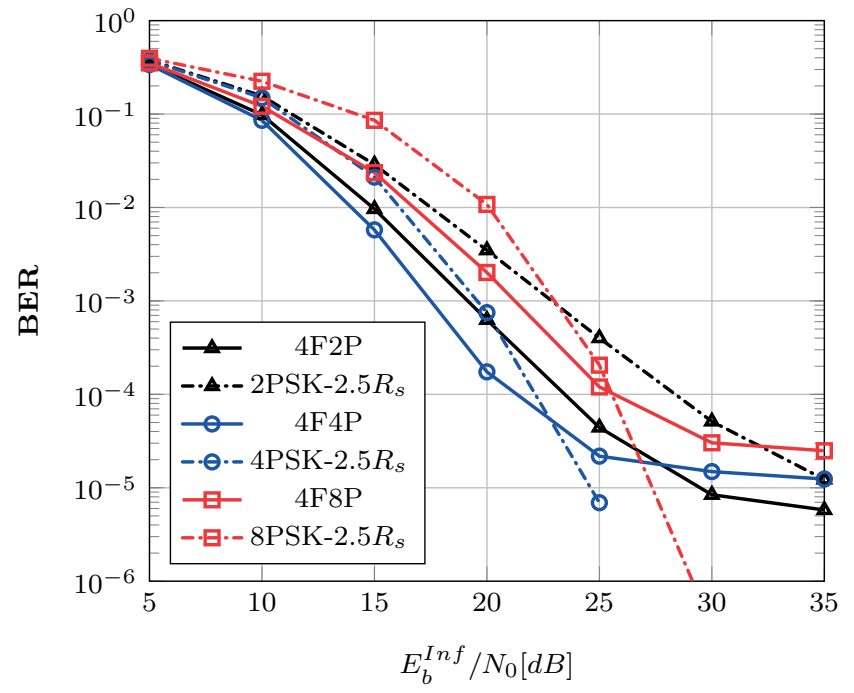

(b)

Figure D.8: BER curves for the system of Figure 6.4 and equal bandwidth scenario. The channel model is Rayleigh with $D_{\max }=5 \mathrm{~Hz}$ and Jakes Doppler spectrum. (a) and (b) show Case I and Case II, respectively. Each marker/color corresponds to one PSK order; triangle (black), circle (blue) and square (red) show 2PSK, 4PSK and 8PSK, respectively. For each color/marker, the solid lines show the hybrid modulation and the dash-dotted lines show the corresponding MPSK modulation with increased symbol rate. 
$D_{\max }=5 \mathrm{~Hz}$ are presented in Figure D.9 and Figure D.10, respectively. Similar trends as mentioned for a Rayleigh channel can be seen for a Rician channel. However, error floors for the hybrid modulation are much lower compared to the Raleigh channel and the BER curve of a fast DDMPSK does not cross the hybrid modulation curves in the range of $\mathrm{BER}>10^{-6}$. In a Rician channel, the best modulation is $4 \mathrm{~F} 4 \mathrm{P}$ which is more than $2 \mathrm{~dB}$ better than the best combination of $4 \mathrm{PSK}$ and higher symbol rate $\left(4 \mathrm{PSK}-2.5 R_{S}\right)$ at $\mathrm{BER}=0.1 \%$. 


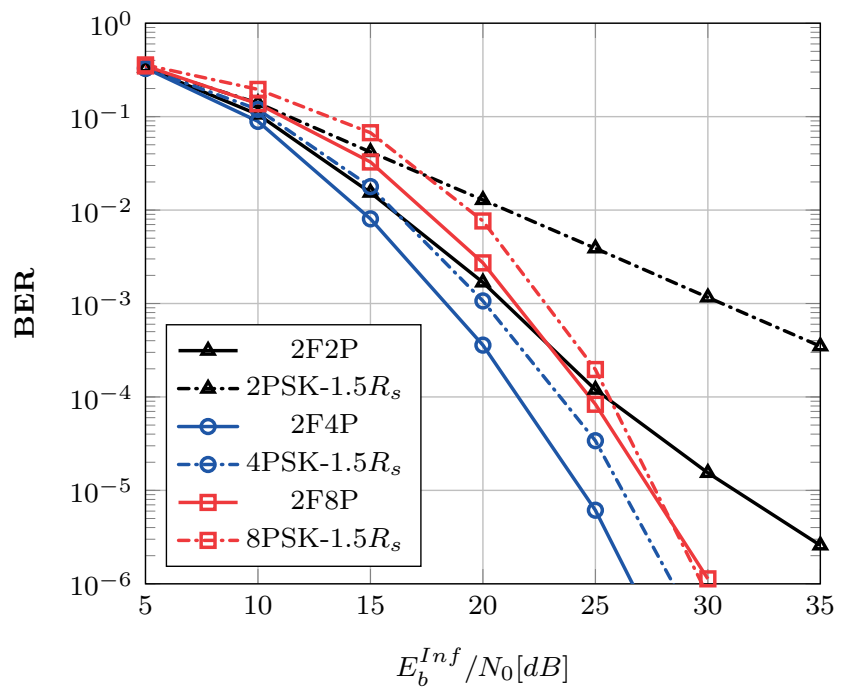

(a)

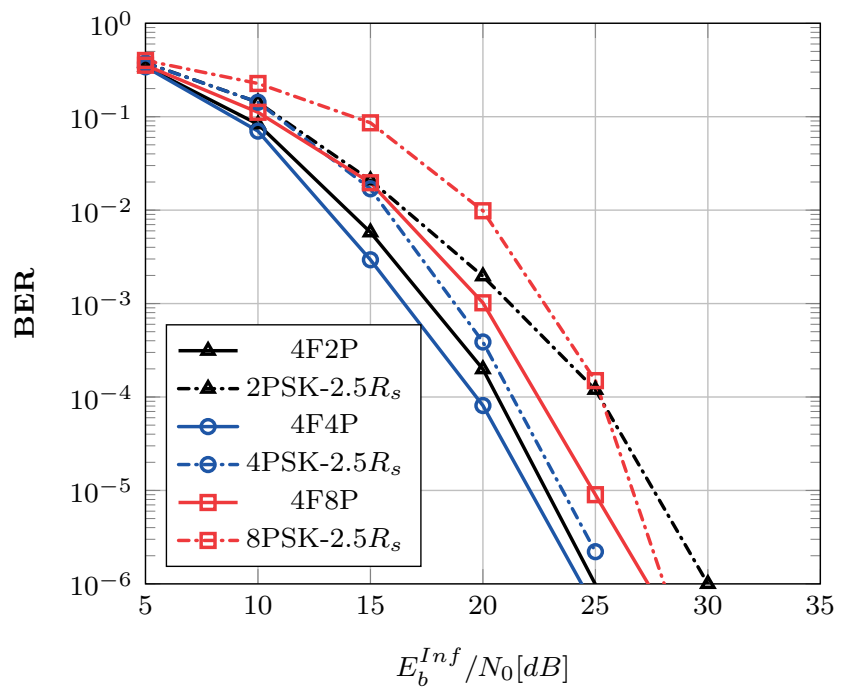

(b)

Figure D.9: BER curves for equal bandwidth scenario Case I (a) and Case II (b). The channel model is Rician $(K=1)$ with $D_{\max }=2 \mathrm{~Hz}$ and rounded Doppler spectrum. Each marker/color corresponds to one PSK order; triangle (black), circle (blue) and square (red) show 2PSK, 4PSK and 8PSK, respectively. For each color/marker, the solid lines show the hybrid modulation and the dash-dotted lines show the corresponding MPSK modulation with increased symbol rate. 


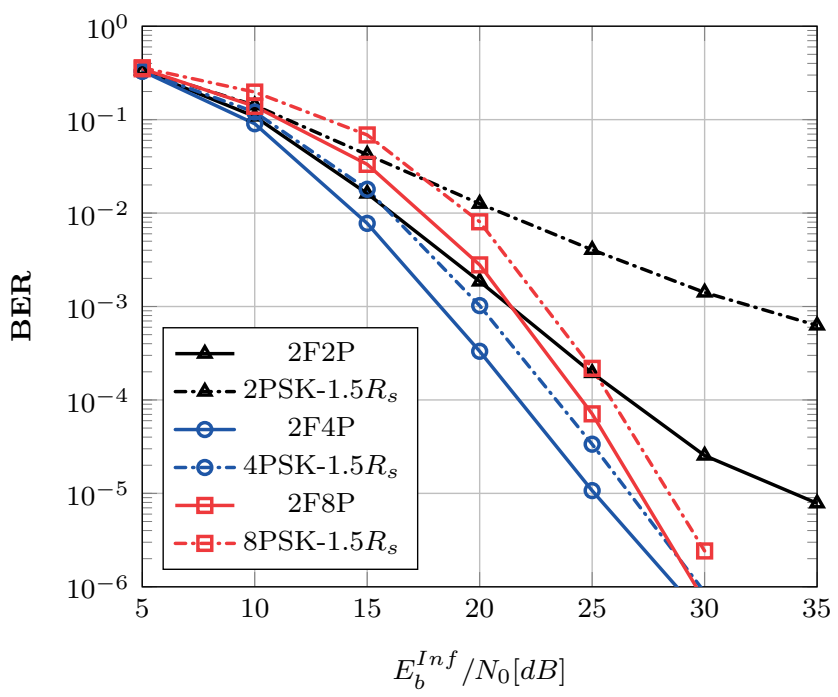

(a)

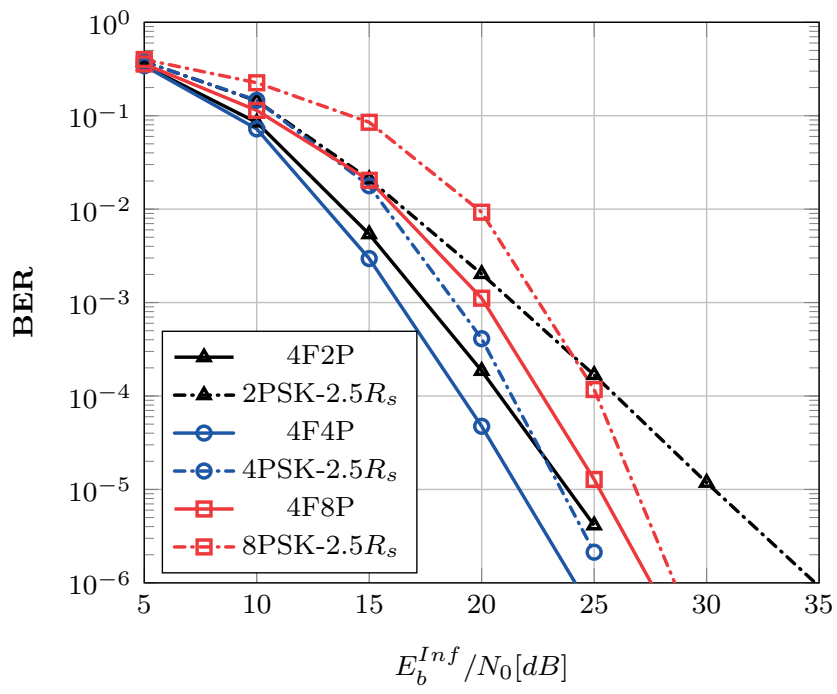

(b)

Figure D.10: BER curves for equal bandwidth scenario Case I (a) and Case II (b). The channel model is Rician $(K=1)$ with $D_{\max }=5 \mathrm{~Hz}$ and rounded Doppler spectrum. Each marker/color corresponds to one PSK order; triangle (black), circle (blue) and square (red) show 2PSK, 4PSK and 8PSK, respectively. For each color/marker, the solid lines show the hybrid modulation and the dash-dotted lines show the corresponding MPSK modulation with increased symbol rate. 


\section{Acronyms}

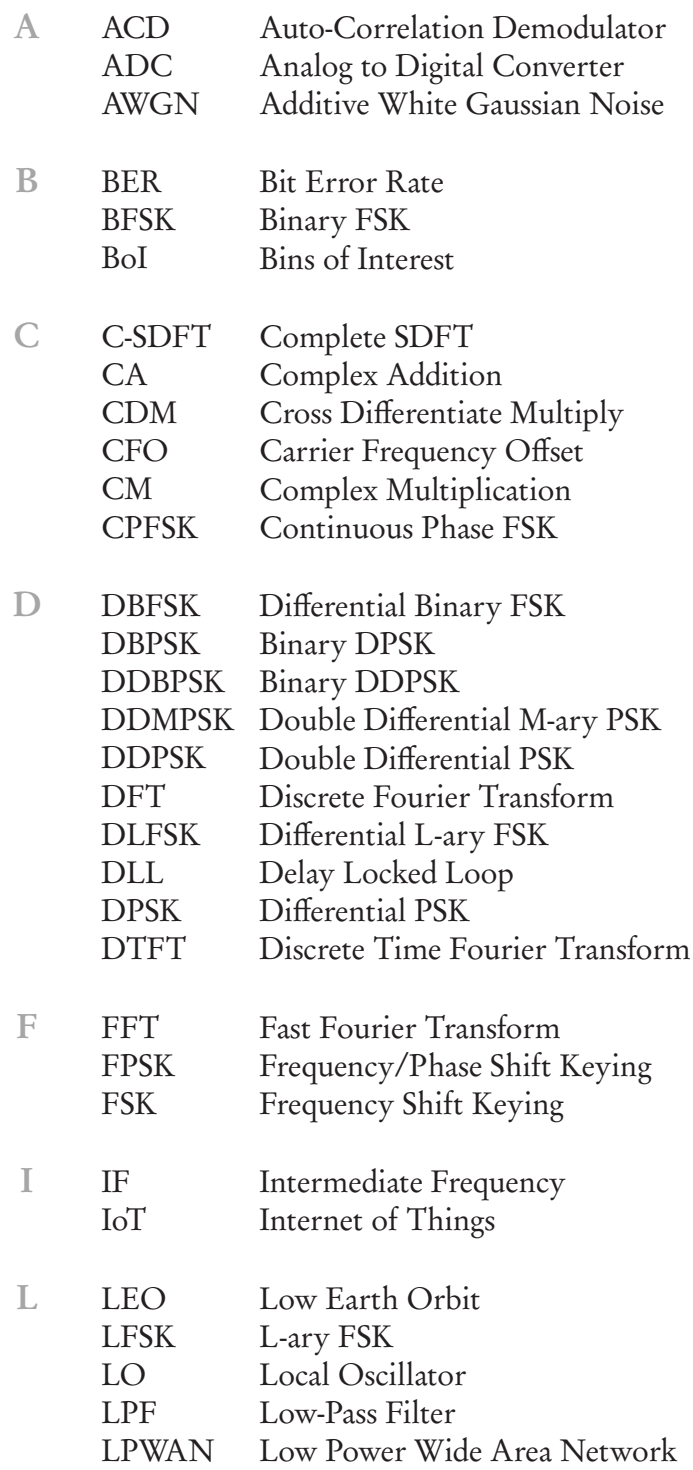




\begin{tabular}{|c|c|c|}
\hline M & $\begin{array}{l}\text { MAC } \\
\text { MAP } \\
\text { MF } \\
\text { MPSK }\end{array}$ & $\begin{array}{l}\text { Medium Access Control } \\
\text { Maximum a Posteriori } \\
\text { Matched Filter } \\
\text { M-ary PSK }\end{array}$ \\
\hline $\mathbf{N}$ & NB-IoT & Narrowband IoT \\
\hline $\mathrm{O}$ & OFDM & Orthogonal Frequency Division Multiplexing \\
\hline $\mathrm{P}$ & PSK & Phase Shift Keying \\
\hline Q & $\begin{array}{l}\mathrm{Q}^{2} \mathrm{PSK} \\
\text { QFPM } \\
\text { QOS } \\
\text { QPSK }\end{array}$ & $\begin{array}{l}\text { Quadrature/Quadrature Phase Shift Keying } \\
\text { Quadrature Frequency/Phase Modulation } \\
\text { Quality of Service } \\
\text { Quadrature PSK }\end{array}$ \\
\hline $\mathrm{R}$ & $\mathrm{RO}$ & Repetition Order \\
\hline S & $\begin{array}{l}\text { SB-SDFT } \\
\text { SDFT } \\
\text { SFFT } \\
\text { SNR } \\
\text { ST-DFT }\end{array}$ & $\begin{array}{l}\text { Single Bin SDFT } \\
\text { Sliding DFT } \\
\text { Sliding FFT } \\
\text { Signal to Noise Ratio } \\
\text { Short-Time Discrete Fourier Transform }\end{array}$ \\
\hline $\mathrm{U}$ & UNB & Ultra-Narrowband \\
\hline V & $\mathrm{VCO}$ & Voltage Controlled Oscillator \\
\hline W & WSN & Wireless Sensor Networks \\
\hline
\end{tabular}




\section{BibliograPHY}

[1] LoRa. Available at https://lora-alliance.org/(2020/08/18). (Cited on page 3).

[2] Sigfox. Available at https://www.sigfox.com/en (2020/08/18). (Cited on pages 3,4 , and 128 ).

[3] Telensa. Available at https://www.telensa.com/ (2020/08/18). (Cited on pages 3 and 4 ).

[4] Weightless. Available at http://www.weightless.org/ (2020/08/18). (Cited on pages 3 and 4 ).

[5] L. Ahumada, R. Feick, and R. A. Valenzuela. Characterization of temporal fading in urban fixed wireless links. IEEE Communications Letters, 10(4):242-244, April 2006. (Cited on page 128).

[6] I. F. Akyildiz, W. Su, Y. Sankarasubramaniam, and E. Cayirci. Wireless sensor networks: a survey. Computer networks, 38(4):393-422, 2002. (Cited on page 1).

[7] A. Al-Fuqaha, M. Guizani, M. Mohammadi, M. Aledhari, and M. Ayyash. Internet of things: A survey on enabling technologies, protocols, and applications. IEEE communications surveys $\mathcal{E}$ tutorials, 17(4):2347-2376, 2015. (Cited on page 1).

[8] G. Anastasi, M. Conti, M. Di Francesco, and A. Passarella. Energy conservation in wireless sensor networks: A survey. Ad hoc networks, 7(3):537-568, 2009. (Cited on page 1).

[9] M. Augustyniak and L. G. Doray. Inference for a leptokurtic symmetric family of distributions represented by the difference of two gamma variates. Journal of Statistical Computation and Simulation, 82(11):1621-1634, 2012. (Cited on page 65).

[10] K. E. Baddour and N. C. Beaulieu. Autoregressive modeling for fading channel simulation. IEEE Transactions on Wireless Communications, 4(4):1650-1662, 2005. (Cited on page 116).

[11] L. M. Borges, F. J. Velez, and A. S. Lebres. Survey on the characterization and classification of wireless sensor network applications. IEEE Communications Surveys E Tutorials, 16(4):1860-1890, 2014. (Cited on page 1).

[12] J. Buetefuer and W. G. Cowley. Frequency offset insensitive multiple symbol detection of MPSK. In 2000 IEEE International Conference on Acoustics, Speech,

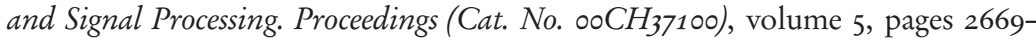
2672. IEEE, 2000. (Cited on pages 54 and 55). 
[13] B. Buurman, J. Kamruzzaman, G. Karmakar, and S. Islam. Low-Power WideArea Networks: Design goals, architecture, suitability to use cases and research challenges. IEEE Access, 8:17179-17220, 2020. (Cited on page 2).

[14] S. Byun, C.-H. Park, Y. Song, S. Wang, C. S. Conroy, and B. Kim. A low-power CMOS bluetooth RF transceiver with a digital offset canceling DLL-based GFSK demodulator. IEEE Journal of Solid-State Circuits, 38(10):1609-1618, 2003. (Cited on pages 6 and 18 ).

[15] K. V. Cartwright and E. J. Kaminsky. An optimum hardware detector for constant envelope quadrature-quadrature phase-shift keying (CEQ/sup 2/PSK). In GLOBECOM 'o5. IEEE Global Telecommunications Conference, 2005., volume 1, pages 4 pp.-, Nov 2005. (Cited on page 21).

[16] C. Char-Dir. Differential detection of quadrature frequency/phase modulated signals. IEEE Transactions on Communications, 47(4):546-557, 1999. (Cited on pages 21 and 115).

[17] Char-Dir Chung and Fu-Chuan Hung. Noncoherent maximum-likelihood block detection of orthogonal NFSK-LDPSK signals. IEEE Transactions on Vebicular Technology, 51(2):283-292, March 2002. (Cited on pages 7 and 106).

[18] P. Chen, B. Zhao, R. Luo, and H. Yang. A low-power all-digital GFSK demodulator with robust clock data recovery. In Proceedings of the great lakes symposium on VLSI, pages 123-128, 2012. (Cited on page 6).

[19] Y.-C. Chen, Y.-C. Wu, and P.-C. Huang. A 1.2-v cmos limiter/rssi/demodulator for low-if fsk receiver. In 2007 IEEE Custom Integrated Circuits Conference, pages 217-220. IEEE, 2007. (Cited on pages 6, 18, and 29).

[20] M. Covell and J. Richardson. A new, efficient structure for the short-time Fourier transform, with an application in code-division sonar imaging. In [Proceedings] ICASSP 91: 1991 International Conference on Acoustics, Speech, and Signal Processing, pages 2041-2044 vol.3. (Cited on pages 76 and 80).

[21] D. Divsalar and M. K. Simon. Multiple-symbol differential detection of MPSK. IEEE Transactions on Communications, 38(3):300-308, Mar 1990. (Cited on page 19).

[22] M.-T. Do, C. Goursaud, and J.-M. Gorce. On the benefits of random FDMA schemes in ultra narrow band networks. In 2014 12th International Symposium on Modeling and Optimization in Mobile, Ad Hoc, and Wireless Networks (WiOpt), pages 672-677. IEEE, 2014. (Cited on page 3).

[23] I. Domuta and T. P. Palade. Sliding DFT and zero padding. In 2019 42nd International Conference on Telecommunications and Signal Processing (TSP), pages 154-157. IEEE, 2019. (Cited on page 83).

[24] S. C. Douglas and J. K. Soh. A numerically-stable sliding-window estimator and its application to adaptive filters. In Conference Record of the Thirty-First Asilomar Conference on Signals, Systems and Computers (Cat. No.97CB36136), volume 1, pages 111-115 vol.1. (Cited on pages 76 and 80). 
[25] D. Duchemin, J.-M. Gorce, and C. Goursaud. Low complexity detector for massive uplink random access with NOMA in IoT LPWA networks. In 2019 IEEE Wireless Communications and Networking Conference Workshop (WCNCW), pages 1-6. IEEE, 2019. (Cited on page 3).

[26] K. Duda. Accurate, guaranteed stable, sliding Discrete Fourier Transform. IEEE Signal Processing Magazine, 27(6):124-127, 2010. ISSN 1053-5888. (Cited on pages $76,80,83,89$, and 90 ).

[27] O. M. El-Ghandour and D. Saha. Differential detection in quadrature-quadrature phase shift keying (Q/sup 2/PSK) systems. IEEE Transactions on Communications, 39(5):703-712, May 1991. (Cited on page 21).

[28] V. Erceg et al. Channel Models for Fixed Wireless Applications. IEEE 802.16 Broadband Wireless Access Working Group, 2001. (Cited on page 129).

[29] A. G. Exposito and J. A. R. Macfas. Fast non-recursive computation of individual running harmonics. IEEE Transactions on Circuits and Systems II: Analog and Digital Signal Processing, 47(8):779-782, 2000. (Cited on pages 76 and 80).

[30] B. Farhang-Boroujeny and S. Gazor. Generalized sliding FFT and its application to implementation of block LMS adaptive filters. IEEE Transactions on Signal Processing, 42(3):532-538, March 1994. (Cited on pages 80, 81, 82, 94, 155, and 157).

[31] B. Farhang-Boroujeny and Y. C. Lim. A comment on the computational complexity of sliding FFT. IEEE Transactions on Circuits and Systems II: Analog and Digital Signal Processing, 39(12):875-876, 1992. (Cited on pages 76 and 80).

[32] M. J. Farooq and Q. Zhu. Optimal dynamic contract for spectrum reservation in mission-critical UNB-IoT systems. In 2018 16th International Symposium on Modeling and Optimization in Mobile, Ad Hoc, and Wireless Networks (WiOpt), pages 1-6. IEEE, 2018. (Cited on page 3).

[33] S. Fleisher and Shouxing Qu. Quadrature frequency/phase modulation. IEEE Transactions on Communications, 43(2/3/4):1513-1524, Feb 1995. (Cited on page 21).

[34] I. Ghareeb. Bit error rate performance and power spectral density of a noncoherent hybrid frequency-phase modulation system. IEEE Journal on Selected Areas in Communications, 13(2):276-284, 1995. (Cited on pages 7, 22, 106, 107, 109, 115, and 125).

[35] I. Ghareeb and A. Yongacoglu. Performance of joint frequency phase modulation over Rayleigh fading channels. IEE Proceedings - Communications, 141(4):241-247, 1994. (Cited on pages 7, 106, 107, 115, and 165).

[36] K. S. Gomadam and S. A. Jafar. Modulation and detection for simple receivers in rapidly time-varying channels. IEEE Transactions on Communications, 55(3): 529-539, 2007. (Cited on pages 115 and 116).

[37] C. Goursaud and J. Gorce. Dedicated networks for IoT: PHY/MAC state of the art and challenges. EAI Endorsed Transactions on Internet of Things, 1(1), 2015. (Cited on page 6). 
[38] C. Goursaud and Y. Mo. Random unslotted time-frequency ALOHA: Theory and application to IoT UNB networks. In 201623 rd International Conference on Telecommunications (ICT), pages 1-5. IEEE, 2016. (Cited on pages 3, 5, 6, 7, and 106).

[39] E. Grayver and B. Daneshrad. A low-power all-digital FSK receiver for space applications. IEEE Transactions on Communications, 49(5):911-921, 2001. (Cited on pages 7,27 , and 30 ).

[40] L. J. Greenstein, S. S. Ghassemzadeh, V. Erceg, and D. G. Michelson. Ricean $k$-factors in narrow-band fixed wireless channels: Theory, experiments, and statistical models. IEEE Transactions on Vehicular Technology, 58(8):4000-4012, Oct 2009. (Cited on page 128).

[41] F. Gu, J. Niu, L. Jiang, X. Liu, and M. Atiquzzaman. Survey of the low power wide area network technologies. Journal of Network and Computer Applications, 149:102459, 2020. (Cited on page 2).

[42] D. A. Gudovskiy and L. Chu. An accurate and stable sliding DFT computed by a modified CIC filter. IEEE Signal Processing Magazine, 34(1):89-93, 2017. (Cited on pages 76 and 80 ).

[43] D. Han and Y. Zheng. A mixed-signal GFSK demodulator based on multithreshold linear phase quantization. IEEE Transactions on Circuits and Systems II: Express Briefs, 56(9):719-723, 2009. (Cited on pages 6, 17, and 29).

[44] S. Hara, A. Wannasarnmaytha, Y. Tsuchida, and N. Morinaga. A novel FSK demodulation method using short-time DFT analysis for LEO satellite communication systems. IEEE Transactions on Vehicular Technology, 46(3):625-633, Aug 1997. (Cited on pages 7, 27, 28, 30, 31, 32, 33, 35, 47, 77, and 112).

[45] S. Haykin. Digital Communication Systems. Wiley, 2013. ISBN 9780471647355. (Cited on pages 14, 15, 16, 38, 44, 45, 118, and 119).

[46] K. Henning. Recommendations for implementing the strategic initiative industrie 4.0. 2013. (Cited on page 1).

[47] N. Instruments. Introduction to Bluetooth device testing: From theory to transmitter and receiver measurements. Technical report, National Instruments, 2016. (Cited on pages 5 and 28 ).

[48] E. Jacobsen and R. Lyons. The sliding DFT. IEEE Signal Processing Magazine, 20 (2):74-80, March 2003. (Cited on pages 76, 80, 82, 83, 89, and 90).

[49] D. W. Jang and T. H. Choi. Decision feedback-based demodulation of doubly differential PSK signals. IEEE Transactions on Communications, 48(8):1253-1256, Aug 2000. (Cited on pages 6 and 54).

[50] H.-S. Kao, M.-J. Yang, and T.-C. Lee. A delay-line-based GFSK demodulator for low-IF receivers. In 2007 IEEE International Solid-State Circuits Conference. Digest of Technical Papers, pages 88-589. IEEE, 2007. (Cited on pages 6, 18, and 29). 
[51] P. Karadimas, E. D. Vagenas, and S. A. Kotsopoulos. On the scatterers' mobility and second order statistics of narrowband fixed outdoor wireless channels. IEEE Transactions on Wireless Communications, 9(7):2119-2124, July 2010. (Cited on page 128).

[52] R. A. Khalona, G. E. Atkin, and J. L. LoCicero. On the performance of a hybrid frequency and phase shift keying modulation technique. IEEE Transactions on Communications, 41(5):655-659, 1993. (Cited on pages 7, 106, 107, and 125).

[53] Z. Kollar, F. Plesznik, and S. Trumpf. Observer-based recursive sliding Discrete Fourier Transform. IEEE Signal Processing Magazine, 35(6):100-106, 2018. (Cited on pages 76,80 , and 82 ).

[54] K. Krishnamoorthy. Handbook of statistical distributions with applications. CRC Press, 2016. (Cited on page 64).

[55] D. Lachartre, F. Dehmas, C. Bernier, C. Fourtet, L. Ouvry, F. Lepin, E. Mercier, S. Hamard, L. Zirphile, S. Thuries, and F. Chaix. 7.5 a TCXO-less $100 \mathrm{~Hz}-$ minimum-bandwidth transceiver for ultra-narrow-band sub-GHz IoT cellular networks. In 2017 IEEE International Solid-State Circuits Conference (ISSCC), pages 134-135, Feb 2017. (Cited on pages 5, 6, 8, 106, and 128).

[56] T.-C. Lee and C.-C. Chen. A mixed-signal GFSK demodulator for Bluetooth. IEEE Transactions on Circuits and Systems II: Express Briefs, 53(3):197-201, 2006. (Cited on pages 6, 16, and 29).

[57] W. Lei and I. Korn. Combined frequency and differential phase shift keying with non-coherent detection in a satellite mobile channel. IEEE Transactions on Vehicular Technology, 44(3):603-612, 1995. (Cited on pages 7, 106, 115, and 125).

[58] H. Leib. Data-aided noncoherent demodulation of DPSK. IEEE transactions on communications, $43(2 / 3 / 4): 722-725,1995$. (Cited on page 20).

[59] W. Lindsey and M. Simón. Telecommunication systems engineering. Prentice-Hall information and system sciences series. Prentice-Hall, 1973. ISBN 9780139024290. (Cited on page 107).

[60] J. Liu, S. Kwatra, and J. Kim. An analysis of decision feedback detection of differentially encoded MPSK signals. IEEE transactions on vehicular technology, 44 (2):261-267, 1995. (Cited on page 20).

[61] E. Lopelli, J. Van der Tang, and A. H. van Roermund. A FSK demodulator comparison for ultra-low power, low data-rate wireless links in ISM bands. In Proceedings of the 2005 European Conference on Circuit Theory and Design, 2005., volume 2, pages II-259. IEEE, 2005. (Cited on pages 29 and 30).

[62] R. W. Ltd. A comparison of UNB and spread spectrum wireless technologies as used in LPWA M2M applications. Technical report, Real Wireless, West Succex, UK, 2015. (Cited on pages 2, 3, and 4). 
[63] M. Z. Mahfouz, A. Meijerink, and M. J. Bentum. Ultra-narrowband for energyscavenging-powered wireless sensor networks. In 2017 IEEE 28th Annual International Symposium on Personal, Indoor, and Mobile Radio Communications (PIMRC), pages 1-7. IEEE, 2017. (Cited on page 4).

[64] P. Mak, S. U, and R. P. Martins. Transceiver architecture selection: Review, stateof-the-art survey and case study. IEEE Circuits and Systems Magazine, 7(2):6-25, 2007. (Cited on page 8).

[65] G. Meciani. Implementation of a power-efficient DFT based demodulator for BFSK (MSc. thesis), December 2018. (Cited on page 155).

[66] K. Mekki, E. Bajic, F. Chaxel, and F. Meyer. A comparative study of LPWAN technologies for large-scale IoT deployment. ICT express, 5(1):1-7, 2019. (Cited on pages 3 and 4 ).

[67] U. Mengali. Synchronization Techniques for Digital Receivers. Applications of Communications Theory. Springer US, 2013. ISBN 9781489918079. (Cited on page 23).

[68] H. Meyr, M. Moeneclaey, and S. Fechtel. Digital Communication Receivers, Volume 2: Synchronization, Channel Estimation, and Signal Processing. Wiley Series in Telecommunications and Signal Processing. Wiley, 1997. ISBN 9780471502753. (Cited on pages 6, 23, and 24).

[69] Y. Mo, C. Goursaud, and J.-M. Gorce. Theoretical analysis of UNB-based IoT networks with path loss and random spectrum access. In 2016 IEEE 27 th Annual International Symposium on Personal, Indoor, and Mobile Radio Communications (PIMRC), pages 1-6. IEEE, 2016. (Cited on page 3).

[70] Y. Mo, C. Goursaud, and J.-M. Gorce. On the benefits of successive interference cancellation for ultra narrow band networks: Theory and application to IoT. In 2017 IEEE International Conference on Communications (ICC), pages 1-6. IEEE, 2017. (Cited on page 3).

[71] A. Molisch. Wireless Communications. Wiley, 2012. ISBN 9781118355688. (Cited on pages $7,105,106,115$, and 130 ).

[72] D.-E. A. Montoya, J. A. Rosendo Macías, and A. Gómez-Expósito. Short-time DFT computation by a modified radix-4 decimation-in-frequency algorithm. Signal Processing, 94:81-89, 2014. (Cited on pages 76, 80, and 81).

[73] D. A. Montoya-Andrade, J. A. Rosendo-Macías, and A. Gómez-Expósito. Efficient computation of the short-time DFT based on a modified radix-2 decimation-infrequency algorithm. Signal Processing, 92(10):2525-2531, 2012. (Cited on pages 76, 80 , and 81 ).

[74] N. Naik. LPWAN technologies for IoT systems: choice between ultra narrow band and spread spectrum. In 2018 IEEE International Systems Engineering Symposium (ISSE), pages 1-8. IEEE, 2018. (Cited on pages 3 and 4). 
[75] R. Ni, K. Mayaram, and T. S. Fiez. A 2.4 GHz hybrid polyphase filter based BFSK receiver with high frequency offset tolerance for wireless sensor networks. IEEE journal of solid-state circuits, 48(5):1250-1263, 2013. (Cited on pages 6 and 29).

[76] K. E. Nolan, W. Guibene, and M. Y. Kelly. An evaluation of low power wide area network technologies for the Internet of Things. In 2016 international wireless communications and mobile computing conference (IWCMC), pages 439-444. IEEE, 2016. (Cited on page 2).

[77] C. Oestges, D. Vanhoenacker-Janvier, and B. Clerckx. Channel characterization of indoor wireless personal area networks. IEEE Transactions on Antennas and Propagation, 54(11):3143-3150, Nov 2006. (Cited on pages 128 and 129).

[78] C. Park. Fast, accurate, and guaranteed stable sliding Discrete Fourier Transform. IEEE Signal Processing Magazine, 32(4):145-156, July 2015. (Cited on pages 76, 80, 81 , and 89 ).

[79] C. Park. Guaranteed-stable sliding DFT algorithm with minimal computational requirements. IEEE Transactions on Signal Processing, 65(20):5281-5288, Oct 2017. (Cited on page 83).

[80] C.-S. Park and S.-J. Ko. The hopping Discrete Fourier Transform. IEEE Signal Processing Magazine, 31(2):135-139, 2014. (Cited on pages 76, 80, and 81).

[81] M. H. Perrott, J. C. Salvia, F. S. Lee, A. Partridge, S. Mukherjee, C. Arft, J. Kim, N. Arumugam, P. Gupta, S. Tabatabaei, et al. A temperature-to-digital converter for a MEMS-based programmable oscillator with $\pm 5 \mathrm{ppm}$ frequency stability and 1 ps integrated jitter. IEEE Journal of Solid-State Circuits, 48(1):276-291, 2012. (Cited on page 5).

[82] J. Proakis and D. Manolakis. Digital Signal Processing. Pearson Prentice Hall, 2007. ISBN 9780131873742. (Cited on page 147).

[83] Q. M. Qadir, T. A. Rashid, N. K. Al-Salihi, B. Ismael, A. A. Kist, and Z. Zhang. Low power wide area networks: a survey of enabling technologies, applications and interoperability needs. IEEE Access, 6:77454-77473, 2018. (Cited on pages 2 and 4 ).

[84] A. M. Rabiei and N. C. Beaulieu. Frequency offset invariant multiple symbol differential detection of MPSK. IEEE Transactions on Communications, 59(3): 652-657, March 2011. (Cited on pages 6, 23, and 54).

[85] Z. Rafii. Sliding Discrete Fourier Transform with kernel windowing [lecture notes]. IEEE Signal Processing Magazine, 35(6):88-92, 2018. (Cited on pages 76 and 80 ).

[86] U. Raza, P. Kulkarni, and M. Sooriyabandara. Low power wide area networks: An overview. IEEE Communications Surveys \& Tutorials, 19(2):855-873, 2017. (Cited on pages 2,3 , and 4 ).

[87] I. Reed and R. Scholtz. N-orthogonal phase-modulated codes. IEEE Transactions on Information Theory, 12(3):388-395, July 1966. (Cited on pages 7, 22, 106, and 107). 
[88] B. Reynders, W. Meert, and S. Pollin. Range and coexistence analysis of long range unlicensed communication. In 201623 rd International Conference on Telecommunications (ICT), pages 1-6. IEEE, 2016. (Cited on page 3).

[89] S. Safapourhajari and A. B. J. Kokkeler. Frequency offset tolerant demodulation for low data rate and narrowband wireless sensor node. In 201711 th International Conference on Signal Processing and Communication Systems (ICSPCS), pages 1-8, Dec 2017. (Cited on pages 35, 77, and 97).

[90] D. Saha and T. G. Birdsall. Quadrature-quadrature phase-shift keying. IEEE Transactions on Communications, 37(5):437-448, May 1989. (Cited on pages 20 and 21).

[91] R. Sanchez-Iborra and M.-D. Cano. State of the art in LP-WAN solutions for industrial IoT services. Sensors, 16(5):708, 2016. (Cited on page 2).

[92] H.-Y. Shih, C.-F. Chen, Y.-C. Chang, and Y.-W. Hu. An ultralow power multirate FSK demodulator with digital-assisted calibrated delay-line based phase shifter for high-speed biomedical zero-IF receivers. IEEE Transactions on Very Large Scale Integration (VLSI) Systems, 23(1):98-106, 2014. (Cited on page 18).

[93] M. Simon, S. Hinedi, and W. Lindsey. Digital Communication Techniques: Signal Design and Detection. Digital Communication Techniques. PTR Prentice Hall, 1995. ISBN 9780132006101. (Cited on pages $38,44,53,55$, and 107).

[94] M. K. Simon and D. Divsalar. On the implementation and performance of single and double differential detection schemes. IEEE Transactions on Communications, 40(2):278-291, Feb 1992. ISSN oogo-6778. (Cited on pages 6, 7, 28, 36, 37, 38, 44, $53,54,55,56,68,111,113$, and 125$)$.

[95] R. S. Sinha, Y. Wei, and S.-H. Hwang. A survey on LPWA technology: LoRa and NB-IoT. Ict Express, 3(1):14-21, 2017. (Cited on page 2).

[96] T. Šolc, T. Gale, and C. Fortuna. Optimization of ultra-narrowband wireless communication: An experimental case study. In 2017 IEEE Conference on Computer Communications Workshops (INFOCOM WKSHPS), pages 523-528. IEEE, 2017. (Cited on page 3).

[97] S. Stein. Fading channel issues in system engineering. IEEE Journal on Selected Areas in Communications, 5(2):68-89, 1987. (Cited on page 120).

[98] P. Stoica, J. Liu, and J. Li. Maximum-likelihood double differential detection clarified. IEEE Transactions on Information Theory, 50(3):572-576, 2004. (Cited on page 54).

[99] P. Stoica, J. Liu, J. Li, and M. A. Prasad. The heuristic, GLRT, and MAP detectors for double differential modulation are identical. IEEE Transactions on Information Theory, 51(5):1860-1865, May 2005. ISSN 0018-9448. (Cited on pages 6 and 54).

[100] J. Wang, B. Woods, and W. F. Eddy. MEG, RFFTs, and the hunt for high frequency oscillations. In 2009 2nd International Congress on Image and Signal Processing, pages $1-5$. (Cited on pages 76,80 , and 81 ). 
[101] J.-M. Wang and W. F. Eddy. Parallel algorithm for SWFFT using 3 D data structure. Circuits, Systems, and Signal Processing, 31(2):711-726, 2011. (Cited on pages 76, 80, and 81 ).

[102] B. Xia, C. Xin, W. Sheng, A. Y. Valero-Lopez, and E. Sánchez-Sinencio. A GFSK demodulator for low-IF Bluetooth receiver. IEEE Journal of solid-state circuits, 38 (8):1397-1400, 2003. (Cited on pages 6, 17, and 29).

[103] F. Xiong. Digital Modulation Techniques. Artech House telecommunications library. Artech House, 2000. ISBN 9780890069707. (Cited on pages 15, 43, 101, and 115).

[104] J. Yick, B. Mukherjee, and D. Ghosal. Wireless sensor network survey. Computer networks, 52(12):2292-2330, 2008. (Cited on page 1).

[105] Y. Yin, Y. Yan, C. Wei, and S. Yang. A low-power low-cost GFSK demodulator with a robust frequency offset tolerance. IEEE Transactions on Circuits and Systems II: Express Briefs, 61(9):696-700, 2014. (Cited on page 17).

[106] M. R. Yuce and W. Liu. A low-power multirate differential PSK receiver for space applications. IEEE Transactions on Vehicular Technology, 54(6):2074-2084, Nov 2005. (Cited on pages 6, 7, and 27).

[107] M. Yuqi, C. Goursaud, and J.-M. Gorce. Uplink multiple base stations diversity for UNB based IoT networks. In 2018 IEEE Conference on Antenna Measurements E Applications (CAMA), pages 1-4. IEEE, 2018. (Cited on page 3). 


\section{List of Publications}

[S:1] S. Safapourhajari and A. B. J. Kokkeler. Offset tolerant hybrid demodulator and time-diversity for ultra-narrowband communications. Manuscript submitted for publication, 2020.

[S:2] Siavash Safapourhajari and André BJ Kokkeler. On the low complexity implementation of the DFT-based BFSK demodulator for ultra-narrowband communications. IEEE Access, 8:146666-146682, 2020.

[S:3] S. Safapourhajari and A. B. J. Kokkeler. Frequency offset tolerant demodulation for low data rate and narrowband wireless sensor node. In 201711 th International Conference on Signal Processing and Communication Systems (ICSPCS), pages 1-8, 2017.

[S:4] S. Safapourhajari and A. B. J. Kokkeler. Demodulation of double differential PSK in presence of large frequency offset and wide filter. In 2018 IEEE 87th Vehicular Technology Conference (VTC Spring), pages 1-5, 2018.

[S:5] S. Safapourhajari and A. B. J. Kokkeler. Offset tolerant demodulator for frequency/phase modulation in time-varying channel. In 2019 IEEE Wireless Communications and Networking Conference (WCNC), pages 1-6, 2019.

[S:6] S. Safapourhajari and A. B. J. Kokkeler. Low complexity synchronization for offset tolerant DFT-based BFSK demodulator. In 2019 IEEE 20th International Workshop on Signal Processing Advances in Wireless Communications (SPAWC), pages 1-5, 2019.

\section{THIS THESIS}

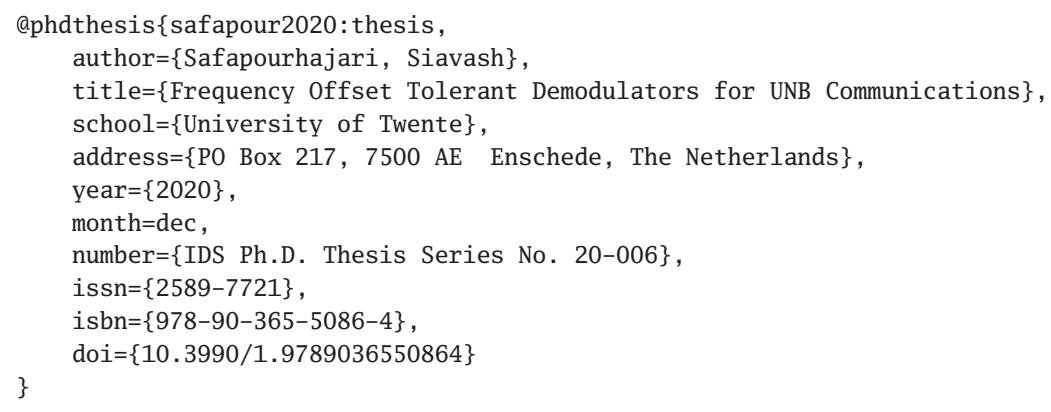

BIB $T_{\mathrm{E}} \mathrm{X}$ of this thesis 


\title{
Propositions
}

accompanying the thesis

\section{Frequency Offset Tolerant Demodulators for UNB Communications}

\author{
by Siavash Safapourhajari,
}

to be defended on vrijdag 11 december 2020

1 - Instead of using costly precise oscillators, demodulation algorithms can be designed such that they tolerate frequency inaccuracy.

(This thesis)

2 - A concept or technique should not be dismissed just because it is not a hot topic anymore. Sometimes adapting old techniques to a new context can bring us interesting solutions.

(This thesis)

3 - In most cases, an algorithm is designed and after that an efficient implementation is devised. Sometimes, it is worth looking at this the other way around; see what efficient implementations exist and modify the algorithm to exploit those implementations.

4 - In academic conferences, poster sessions are more efficient than parallel oral presentation sessions. Presenters and audience match better and fruitful discussions are stimulated.

5 - Technical writing should be treated as an essential tool for students not just a luxury addition to engineering education.

6 - To have a worthwhile outcome from thinking out of the box, a person should first know where the box is and why it is there.

7 - The advantage of tolerance for human societies is undebatable. The crucial challenge is determining the boundaries to avoid censorship of tolerant people by intolerant ones.

8 - When integrating into a new society, it is helpful to understand how the people of that society think and try to see the world from their eyes. In this way, cultural components which were once surprises might become new norms. 

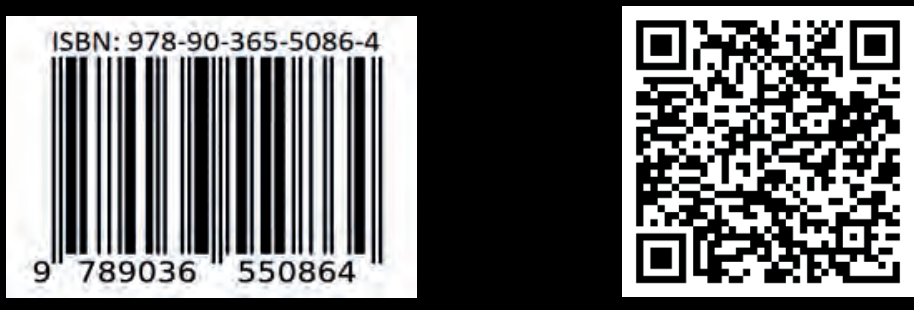